\section{Pacific Northwest}

National Laboratory

Operated by Battelle for the

U.S. Department of Energy

\title{
Subsurface Transport Over Reactive Multiphases (STORM): A Parallel, Coupled, Nonisothermal Multiphase Flow, Reactive Transport, and Porous Medium Alteration Simulator, Version 3.0
}

User’s Guide

D. H. Bacon

M. D. White

B. P. McGrail

July 2004

Prepared for the U.S. Department of Energy under Contract DE-AC06-76RL01830 


\title{
DISCLAIMER
}

This report was prepared as an account of work sponsored by an agency of the United States Government. Neither the United States Government nor any agency thereof, nor Battelle Memorial Institute, nor any of their employees, makes any warranty, express or implied, or assumes any legal liability or responsibility for the accuracy, completeness, or usefulness of any information, apparatus, product, or process disclosed, or represents that its use would not infringe privately owned rights. Reference herein to any specific commercial product, process, or service by trade name, trademark, manufacturer, or otherwise does not necessarily constitute or imply its endorsement, recommendation, or favoring by the United States Government or any agency thereof, or Battelle Memorial Institute. The views and opinions of authors expressed herein do not necessarily state or reflect those of the United States Government or any agency thereof.

\author{
PACIFIC NORTHWEST NATIONAL LABORATORY \\ operated by \\ BATTELLE \\ for the \\ UNITED STATES DEPARTMENT OF ENERGY \\ under Contract DE-AC06-76RLO 1830 \\ Printed in the United States of America \\ Available to DOE and DOE contractors from the \\ Office of Scientific and Technical Information, P.O. Box 62, Oak Ridge, TN 37831; \\ prices available from (615) 576-8401. \\ Available to the public from the National Technical Information Service, \\ U.S. Department of Commerce, 5285 Port Royal Rd., Springfield, VA 22161
}

This document was printed on recycled paper. 
Subsurface Transport over Reactive Multiphases (STORM): A Parallel, Coupled, Nonisothermal Multiphase Flow, Reactive Transport, and Porous Medium Alteration Simulator, Version 3.0

\section{User's Guide}

D. H. Bacon

M. D. White

B. P. McGrail

July 2004

Prepared for

the U.S. Department of Energy

under Contract DE-AC06-76RLO 1830

Pacific Northwest National Laboratory

Richland, Washington 99352 


\section{Summary}

The U.S. Department of Energy must approve a performance assessment (PA) to support the design, construction, approval, and closure of disposal facilities for immobilized low-activity waste (ILAW) currently stored in underground tanks at Hanford, Washington. A critical component of the PA is to provide quantitative estimates of radionuclide release rates from the engineered portion of the disposal facilities. Computer simulations are essential for this purpose because impacts on groundwater resources must be projected to periods of 10,000 years and longer. The computer code selected for simulating the radionuclide release rates is the Subsurface Transport Over Reactive Multiphases (STORM) simulator.

The STORM simulator solves coupled conservation equations for component mass and energy that describe subsurface flow over aqueous and gas phases through variably saturated geologic media. The resulting flow fields are used to sequentially solve conservation equations for reactive aqueous phase transport through variably saturated geologic media. These conservation equations for component mass, energy, and solute mass are partial differential equations that mathematically describe flow and transport through porous media.

The STORM simulator solves the governing-conservation equations and constitutive functions using numerical techniques for nonlinear systems. The partial differential equations governing thermal and fluid flow processes are solved by the integral volume finite difference method. These governing equations are solved simultaneously using Newton-Raphson iteration. The partial differential equations governing reactive solute transport are solved using either an operator split technique where geochemical reactions and solute transport are solved separately, or a fully coupled technique where these equations are solved simultaneously.

The STORM simulator is written in the FORTRAN 77 language, following American National Standards Institute (ANSI) standards. The simulator utilizes a variable source code configuration that allows tailoring of the execution memory and speed to the specific problem by editing text parameter files and recompiling the code. Execution of the STORM simulator is controlled through a text input file. This input file uses a structured format of associated groups of input data. Input data files and results are also presented for model verification and example simulations. Appendix A to this report presents examples of the STORM Version 3.0 input data format for each of the groups.

A parallel version of the STORM simulator, STORM1m, may be compiled to run on any number of multiple processors. Calls to the Message Passing Interface (MPI) libraries have been written directly into the FORTRAN 77 code. Scripts for compiling and running on systems using the Linux operating system are provided in Chapter 6 of this report. 


\section{Acknowledgments}

The authors thank Vicky Freedman of Pacific Northwest National Laboratory (PNNL) for her detailed technical review of this report. We also thank Dr. Fred Mann of CH2M HILL, Inc., for providing continuing support for the development of STORM. This research was performed in part using the Molecular Science Computing Facility (MSCF) in the William R. Wiley Environmental Molecular Sciences Laboratory, a national scientific user facility sponsored by the U.S. Department of Energy's Office of Biological and Environmental Research and located at PNNL. PNNL is operated for the U.S. Department of Energy by Battelle. 


\section{Acronyms and Abbreviations}

$\begin{array}{ll}\text { ANSI } & \text { American National Standards Institute } \\ \text { DOE } & \text { Department of Energy } \\ \text { Ecology } & \text { Washington State Department of Ecology } \\ \text { Eh } & \text { electrode potential in volts } \\ \text { EMSL } & \text { Environmental and Molecular Sciences Laboratory } \\ \text { L } & \text { length } \\ \text { IDF } & \text { integrated disposal facility } \\ \text { ILAW } & \text { immobilized low activity waste } \\ \text { LAW } & \text { low activity waste } \\ \text { M } & \text { mass } \\ \text { MSCF } & \text { Molecular Science Computing Facility } \\ \text { N/A } & \text { not applicable } \\ \text { ODE } & \text { ordinary differential equation } \\ \text { PA } & \text { performance assessment } \\ \text { PDE } & \text { partial differential equation } \\ \text { PNNL } & \text { Pacific Northwest National Laboratory } \\ \text { PUF } & \text { Pressurized Unsaturated Flow (experiments) } \\ \text { SI } & \text { Systeme Internationale } \\ \text { STDIO } & \text { standard input/output device } \\ \text { STOMP } & \text { Subsurface Transport Over Multiple Phases } \\ \text { STORM } & \text { Subsurface Transport Over Reactive Multiphases } \\ \text { STORM1 } & \text { STORM mode with unsaturated flow } \\ \text { STORM1m } & \text { Parallel STORM mode with unsaturated flow } \\ \text { STORM2 } & \text { STORM mode with two-phase flow } \\ \text { STORM3 } & \text { STORM mode with two-phase flow and heat transfer } \\ \text { T } & \text { time } \\ \text { TVD } & \text { Total Variation Diminishing (transport scheme) } \\ & \end{array}$




\section{Contents}

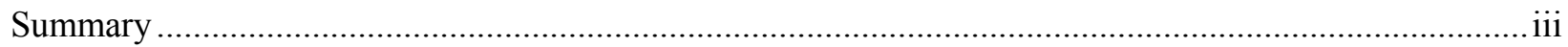

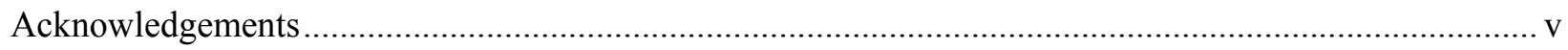

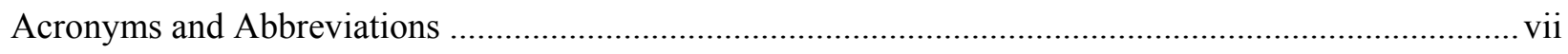

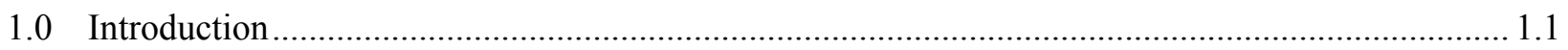

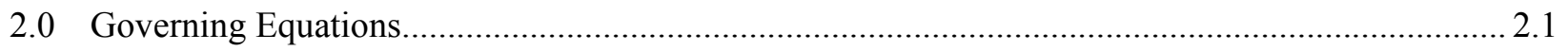

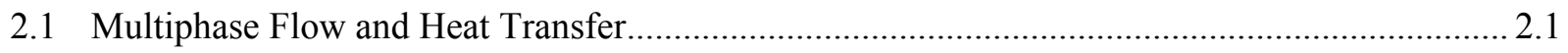

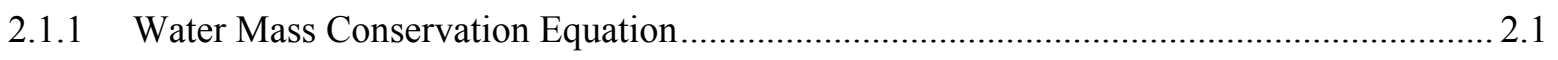

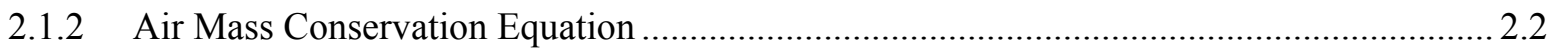

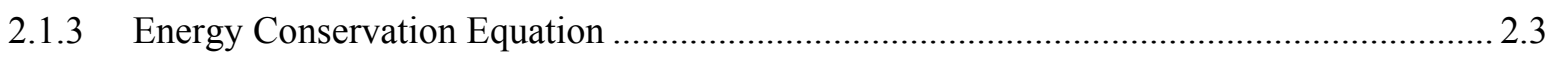

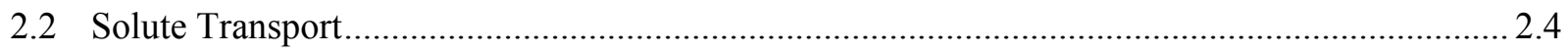

2.2.1 Solute Mass Conservation Equation ............................................................................. 2.4

2.2.2 Aqueous Species Mass Conservation Equation ............................................................... 2.4

2.2.3 Coupled Aqueous and Gaseous Species Mass Conservation Equation .............................. 2.5

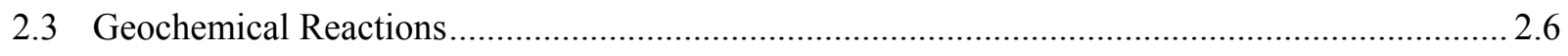

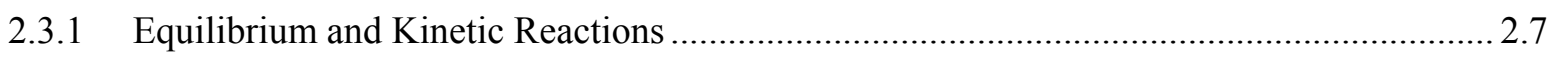

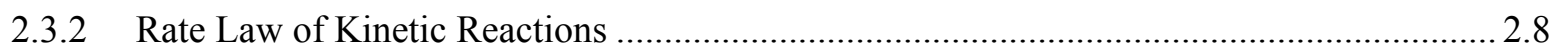

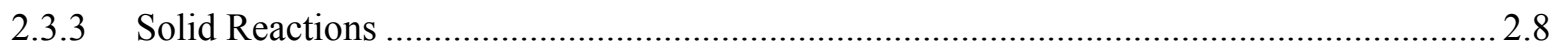

2.4 Coupling Between Fluid Flow and Geochemical Reactions........................................... 2.10

2.4.1 Porosity Changes Coupled with Multiphase Flow ......................................................... 2.10

2.4.2 Water Consumption in Chemical Reactions Coupled with Multiphase Flow................... 2.10

2.4.3 Gas Consumption in Chemical Reactions Coupled with Multiphase Flow ...................... 2.11

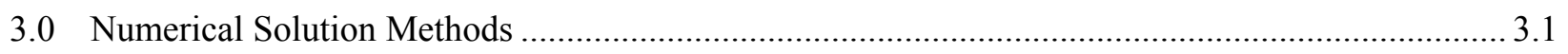

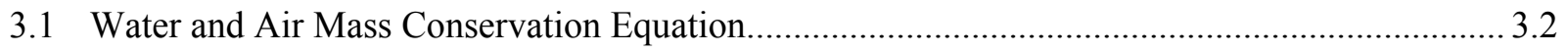

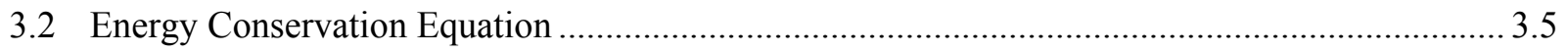

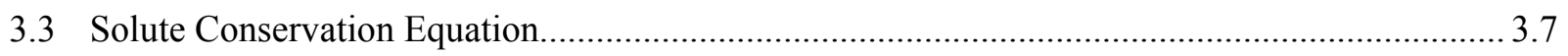

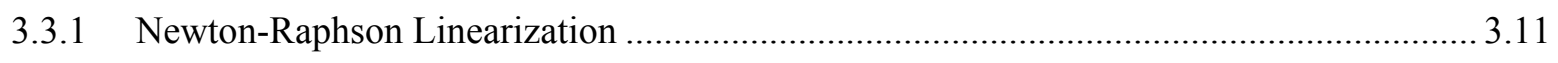

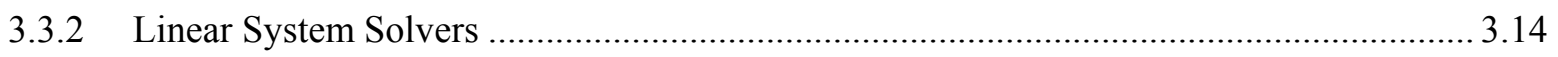

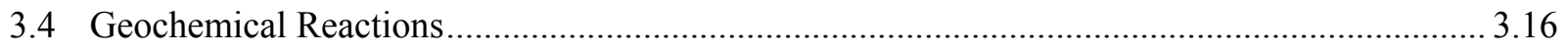

3.4.1 Linear Transformation of the Reaction-Transport Equation............................................ 3.17

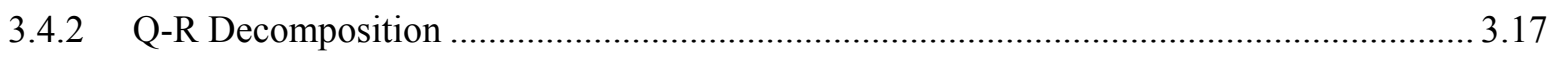

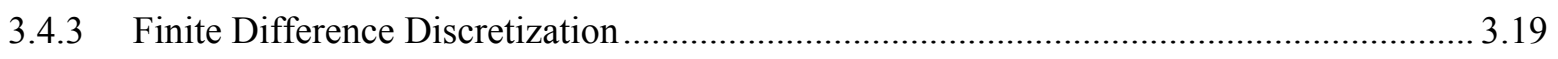

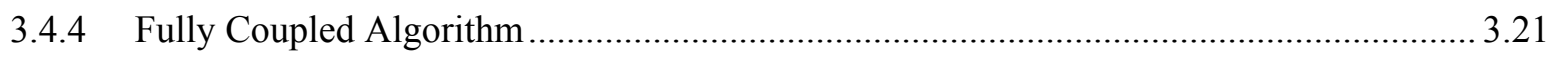




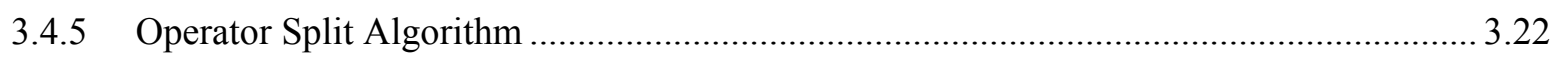

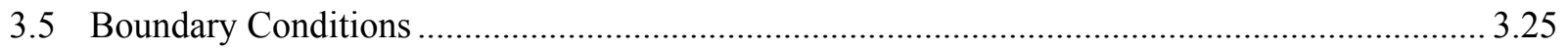

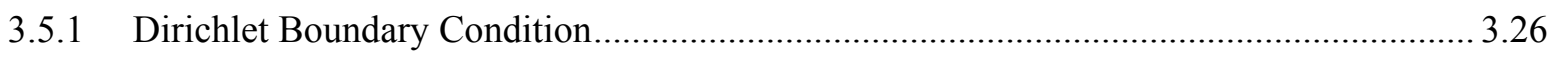

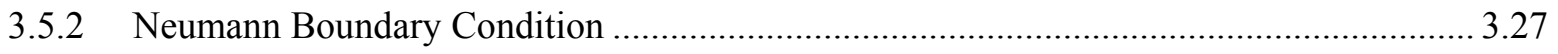

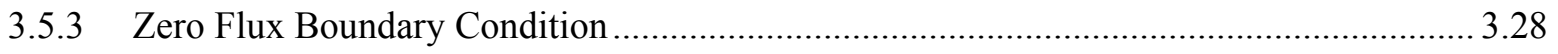

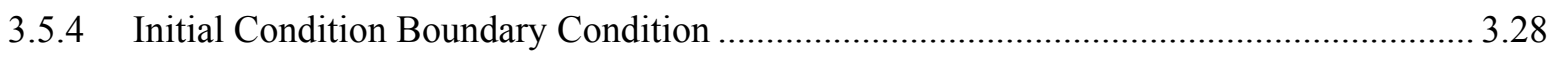

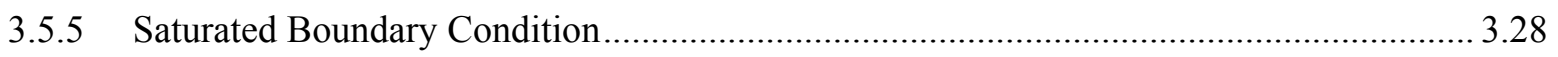

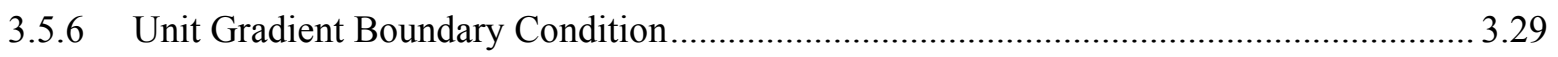

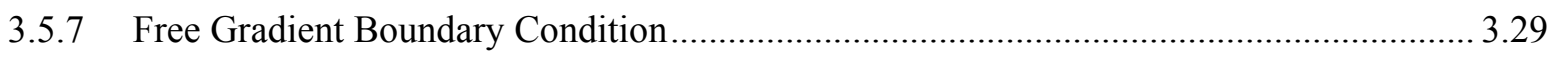

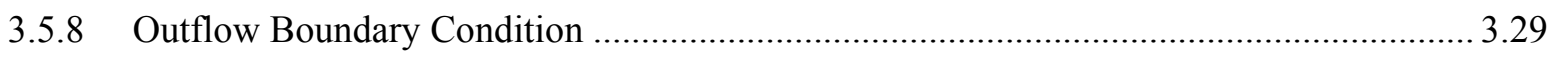

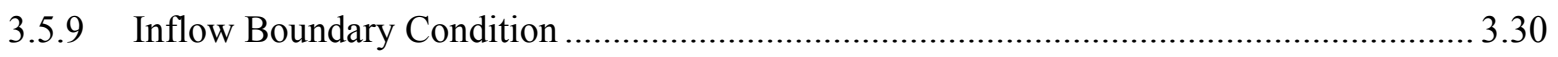

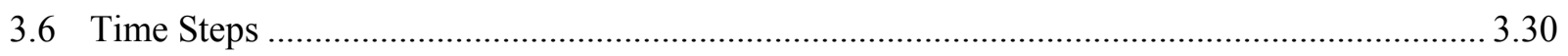

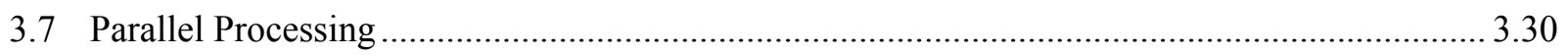

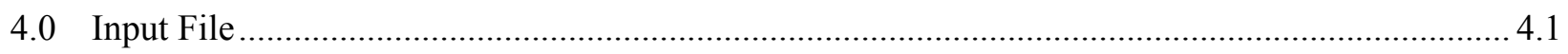

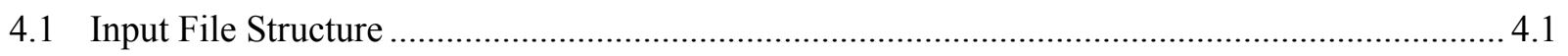

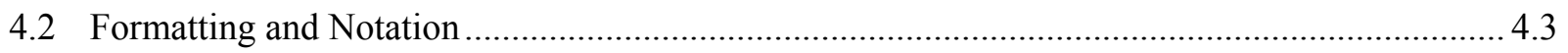

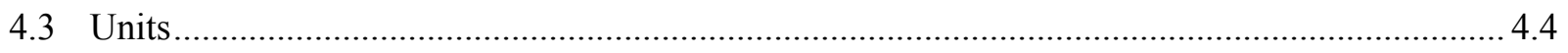

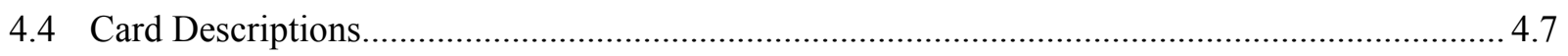

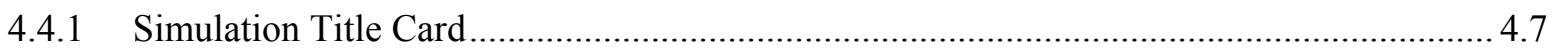

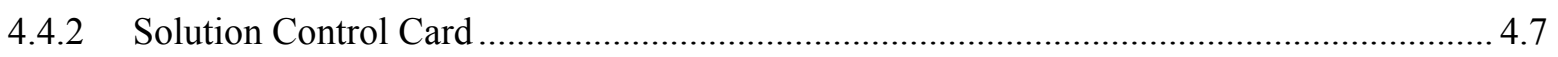

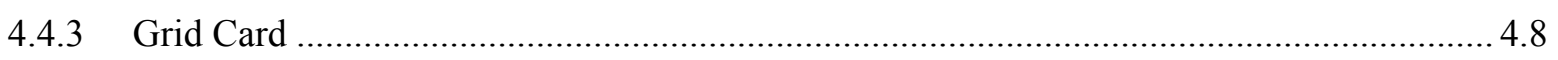

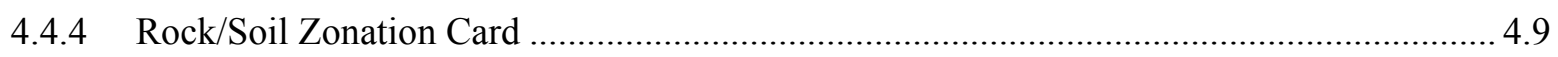

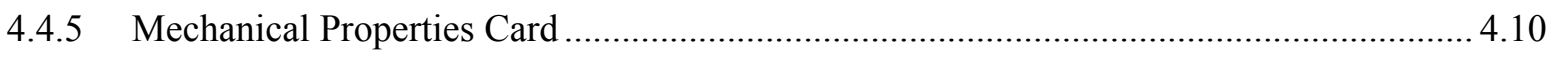

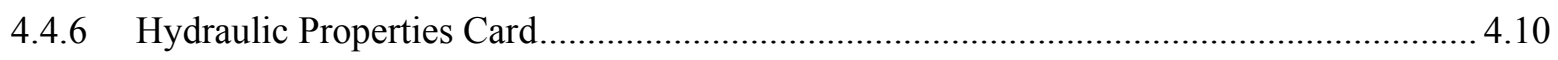

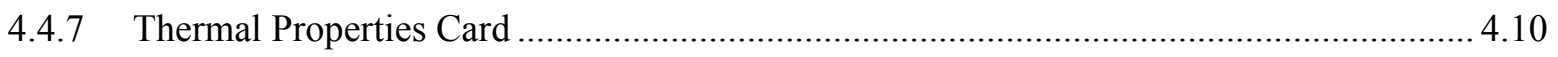

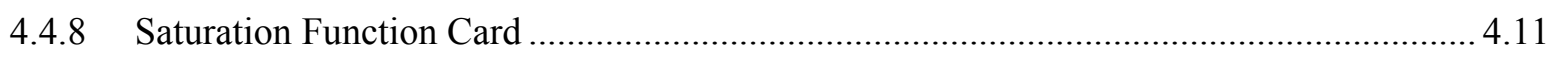

4.4.9 Aqueous Relative Permeability Function Card .......................................................... 4.11

4.4.10 Gas Relative Permeability Function Card .................................................................. 4.11

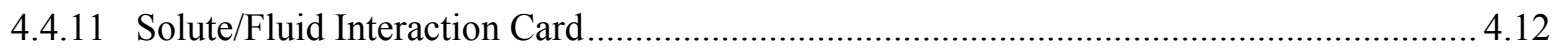

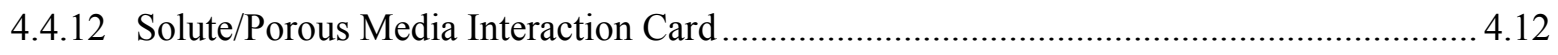

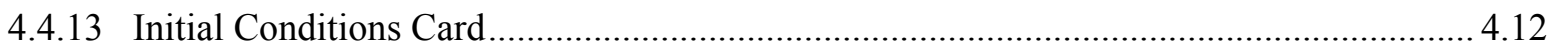

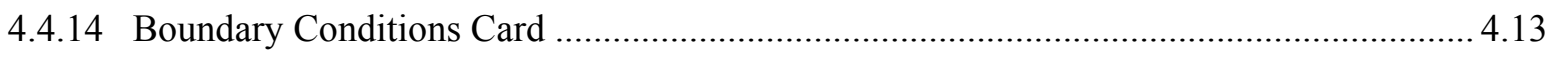

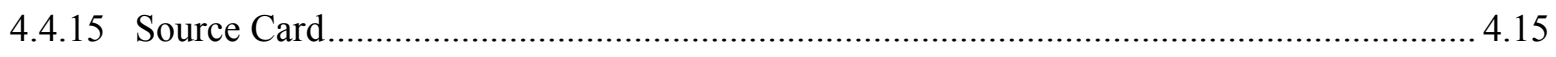

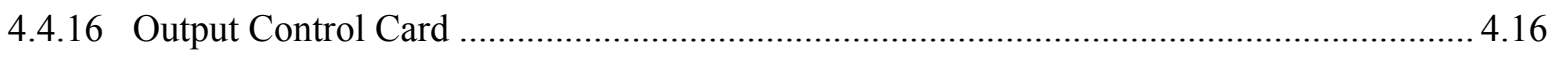




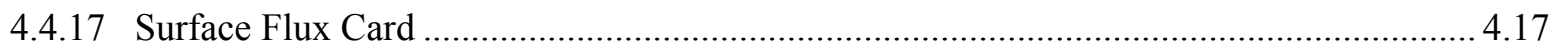

4.4.18 Reactive Transport Control Card …............................................................................... 4.17

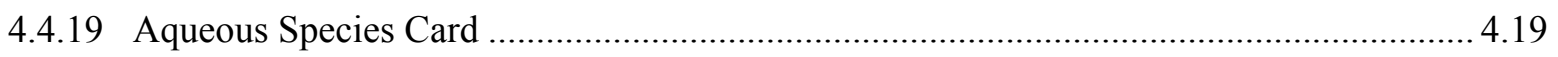

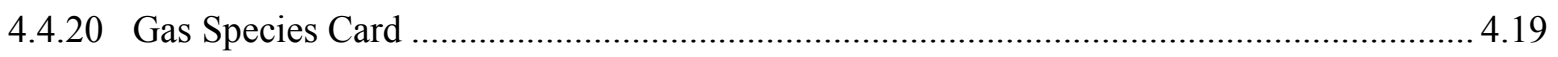

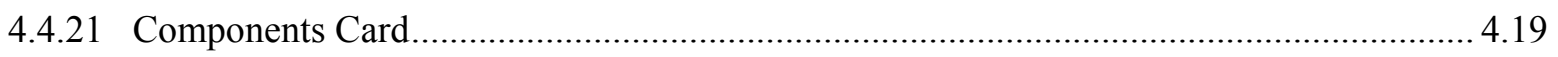

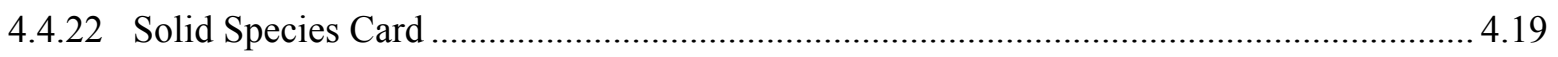

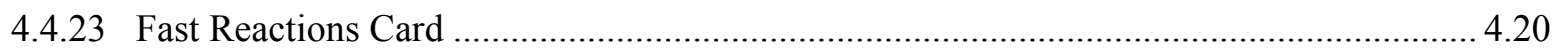

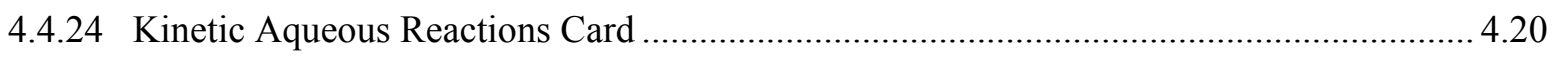

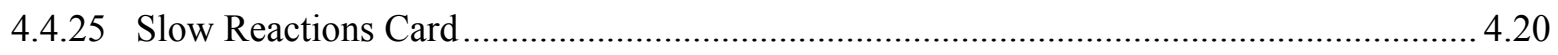

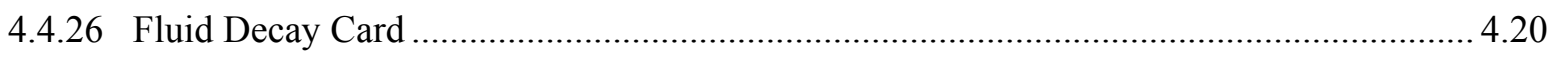

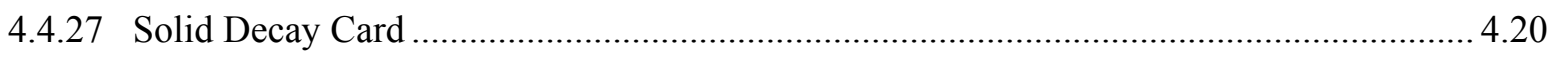

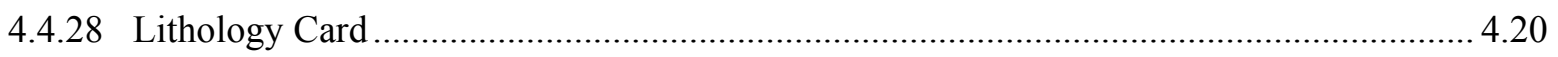

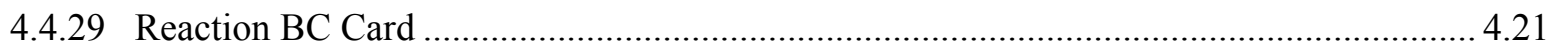

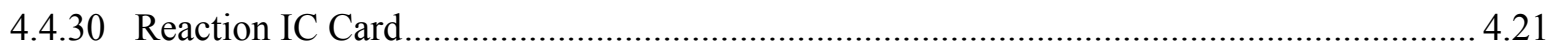

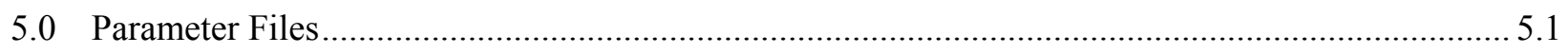

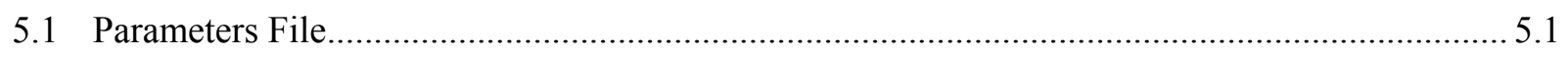

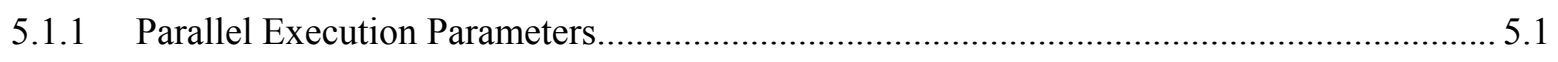

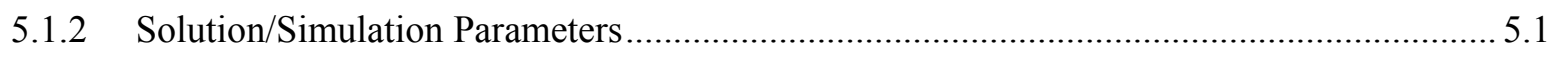

5.1.3 Computational Domain Parameters ............................................................................. 5.1

5.1.4 Operation Mode Switch Parameters............................................................................... 5.2

5.1.5 Linear System Solver Switch Parameters ..................................................................... 5.2

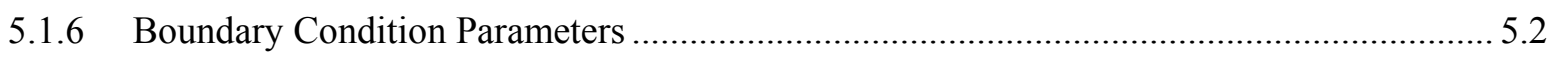

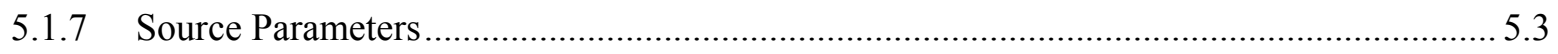

5.1.8 Rock Type and Solute Transport Parameters................................................................ 5.3

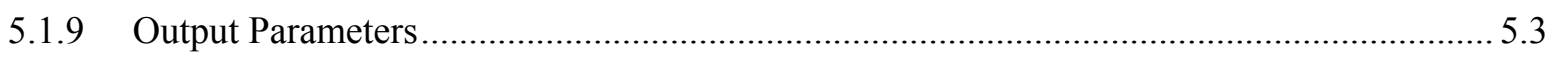

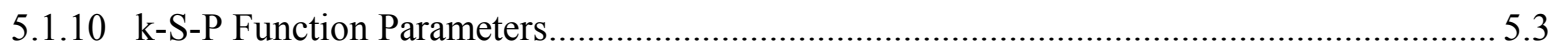

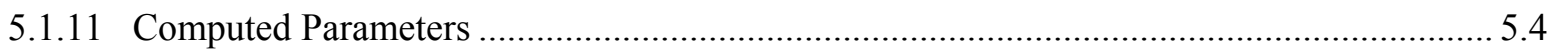

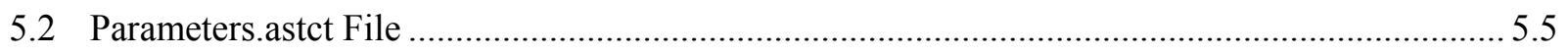

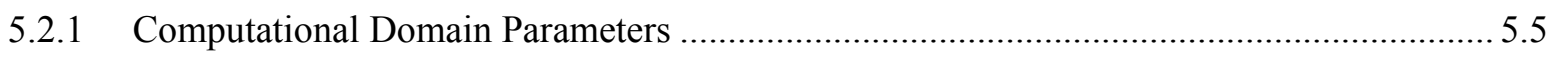

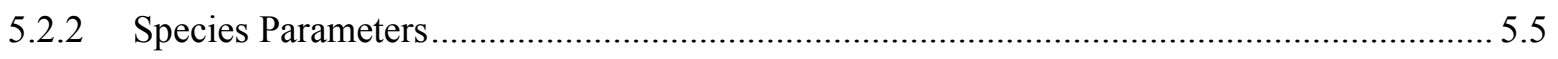

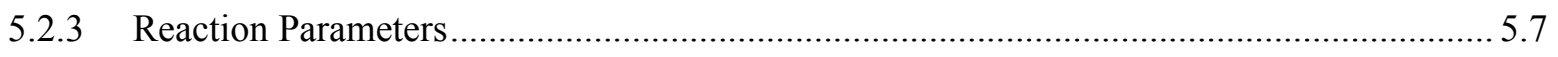

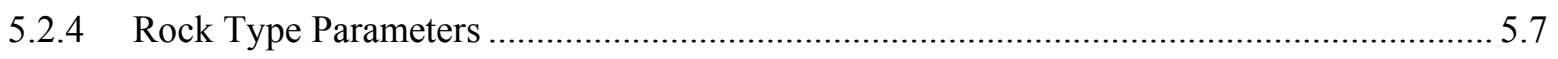

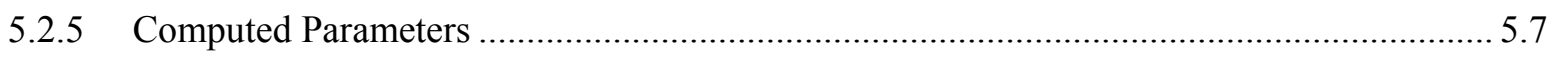

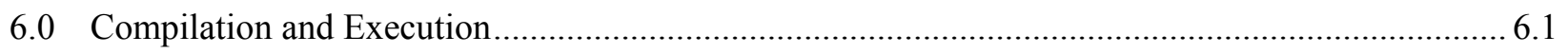




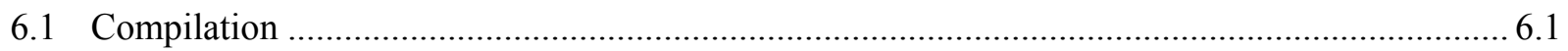

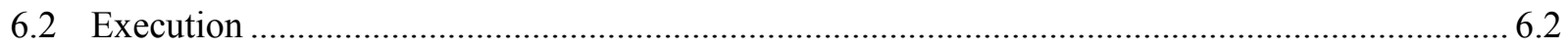

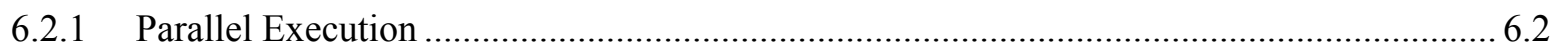

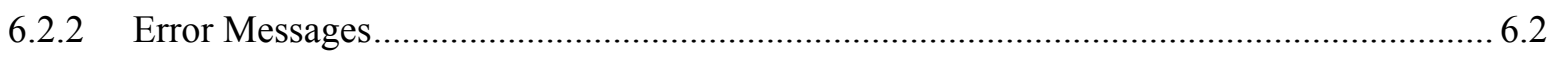

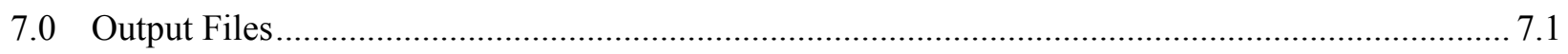

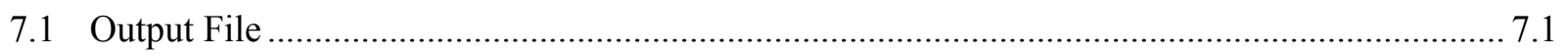

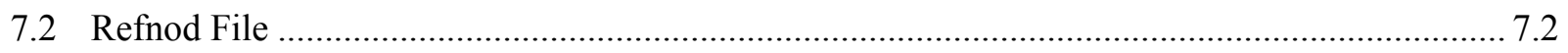

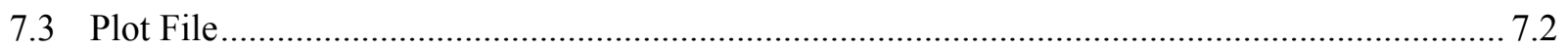

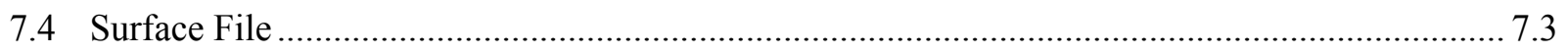

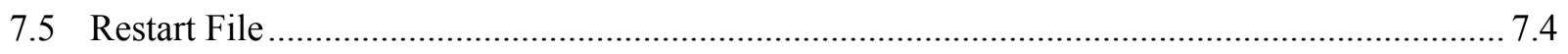

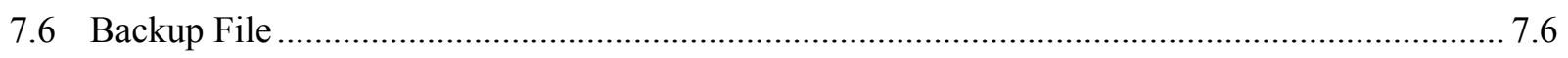

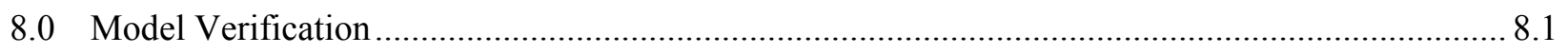

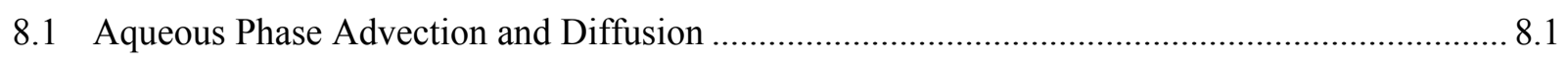

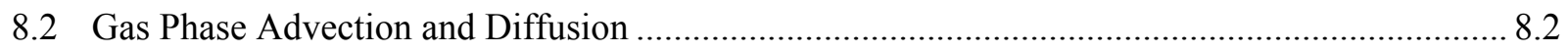

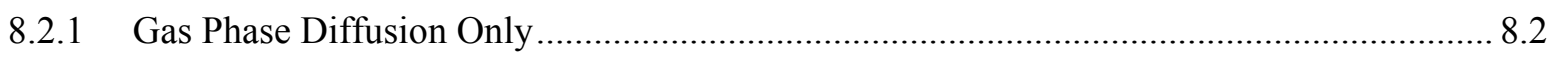

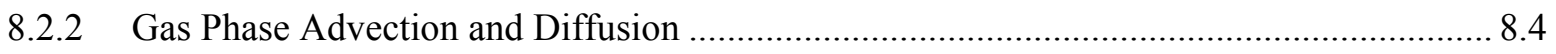

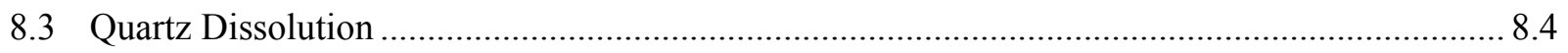

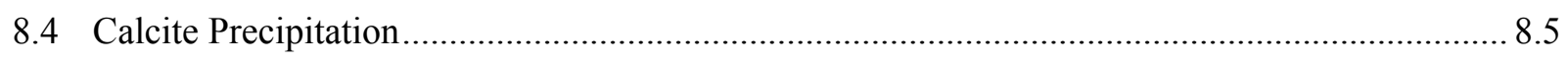

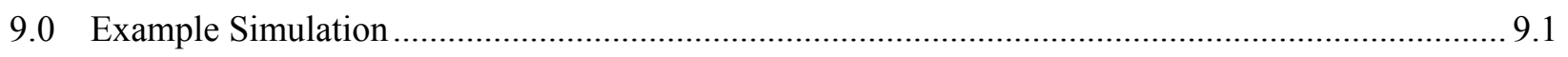

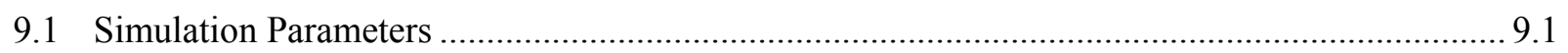

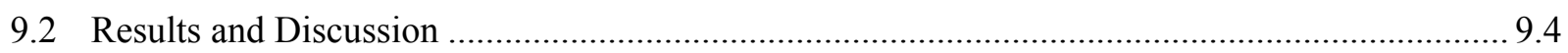

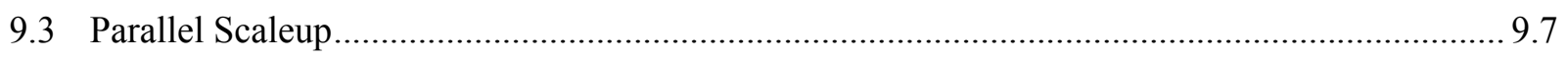

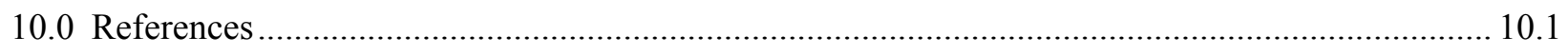

Appendix A: STORM Version 3.0 Input Data Format ................................................................. A.1

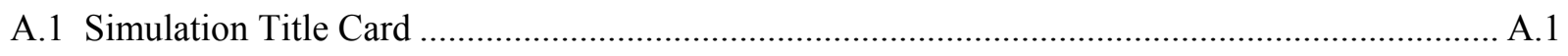

A.1.1 Simulation Title Card Example ............................................................................... A.2

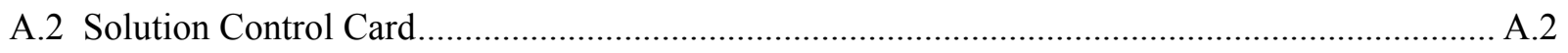

A.2.1 Solution Control Card Example …............................................................................ A.4

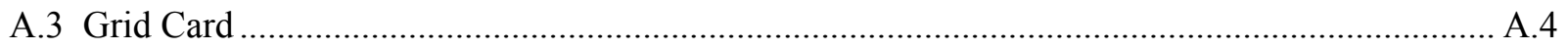

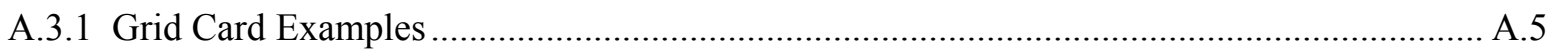

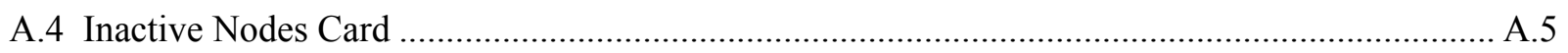

A.4.1 Inactive Nodes Card Examples ............................................................................... A.6

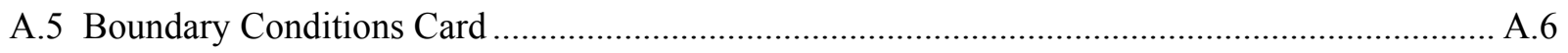

A.5.1 Boundary Conditions Card Examples …................................................................... A.9 


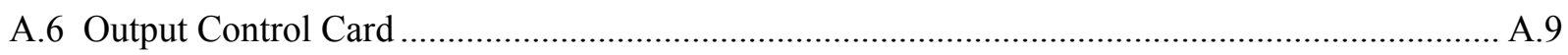

A.6.1 Reference Node Variable and Plot File Variable Options............................................... A.10

A.6.2 Reference Node Variable and Plot File Variable Options................................................. A.10

A.6.3 Reference Node Variable and Plot File Variable Options................................................. A.11

A.6.4 Default Units for the Reference Node and Plot File Variables .......................................... A.12

A.6.5 Output Control Card Example....................................................................................... A.12

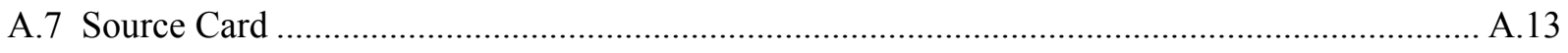

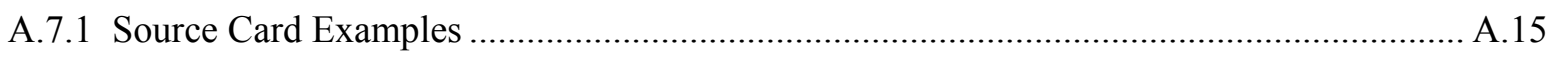

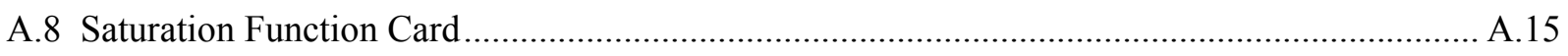

A.8.1 Saturation Function Card Examples.......................................................................... A.17

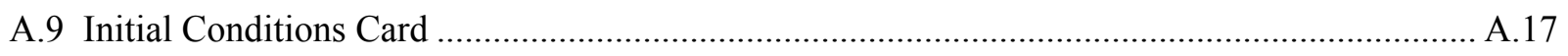

A.9.1 Initial Conditions Card Examples ............................................................................. A.19

A.10 Mechanical Properties Card ............................................................................................ A.20

A.10.1 Mechanical Properties Card Examples........................................................................ A.21

A.11 Hydraulic Properties Card .......................................................................................... A.21

A.11.1 Hydraulic Properties Card Examples ....................................................................... A.22

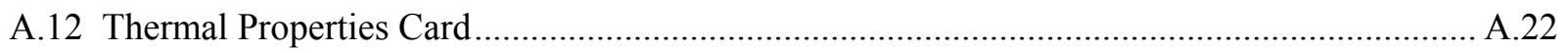

A.12.1 Thermal Properties Card Examples......................................................................... A.23

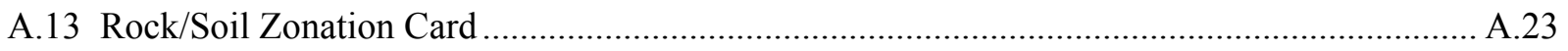

A.13.1 Rock/Soil Zonation Card Examples ....................................................................... A.24

A.14 Aqueous Relative Permeability Card................................................................................ A.24

A.14.1 Aqueous Relative Permeability Card Examples ......................................................... A.25

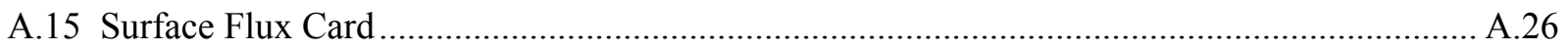

A.15.1 Surface Flux Card Example .................................................................................. A.27

A.16 Gas Relative Permeability Card..................................................................................... A.27

A.16.1 Gas Relative Permeability Card Examples ............................................................. A.28

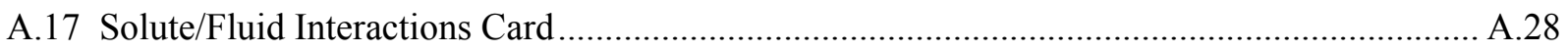

A.17.1 Solute/Fluid Interactions Card Examples................................................................ A.30

A.18 Solute/Porous Media Interactions Card ............................................................................... A.31

A.18.1 Solute/Porous Media Interaction Card Examples ....................................................... A. 31

A.19 Reactive Transport Control Card ....................................................................................... A.32

A.19.1 Reactive Transport Control Card Example ................................................................. A.33

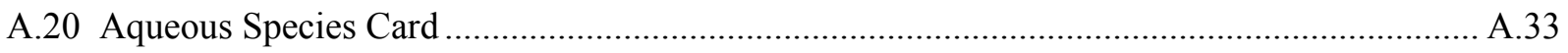

A.20.1 Aqueous Species Card Example ….......................................................................... A.34 


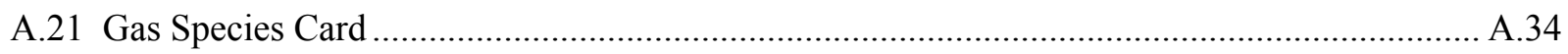

A.21.1 Gas Species Card Example ...................................................................................... A. 35

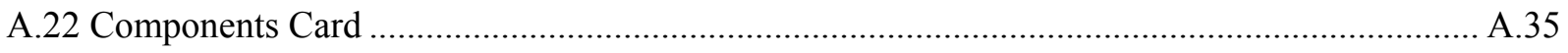

A.22.1 Components Card Example...................................................................................... A.36

A.23 Solid Species Card ......................................................................................................... A.36

A.23.1 Solid Species Card Example .................................................................................... A.36

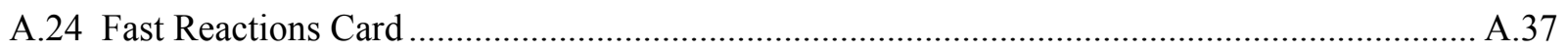

A.24.1 Fast Reactions Card Example .............................................................................. A. 37

A.25 Kinetic Aqueous Reactions Card ................................................................................. A.38

A.25.1 Kinetic Aqueous Reactions Card Example ................................................................ A.3

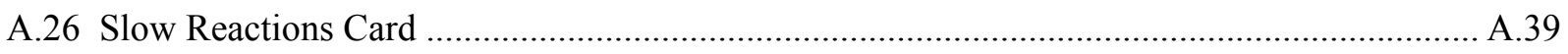

A.26.1 Slow Reactions Card Example .................................................................................. A.39

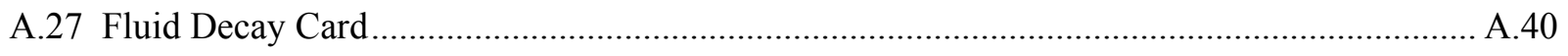

A.27.1 Fluid Decay Card Example …................................................................................. A.40

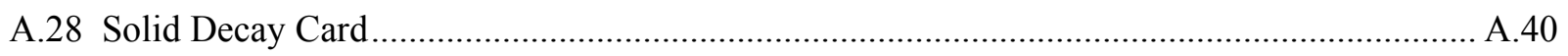

A.28.1 Solid Decay Card Example ................................................................................... A.41

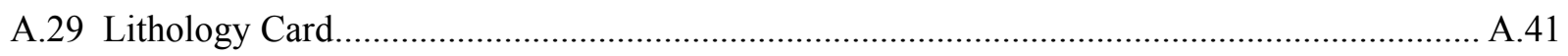

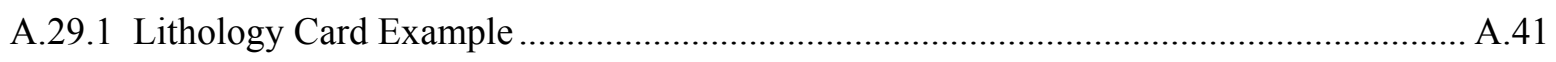

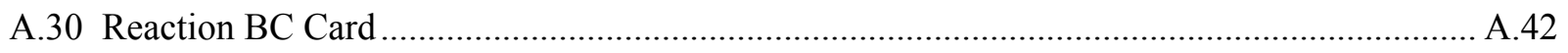

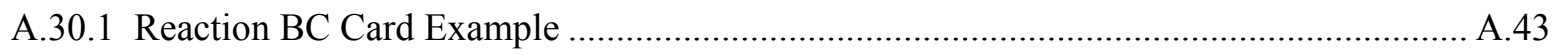

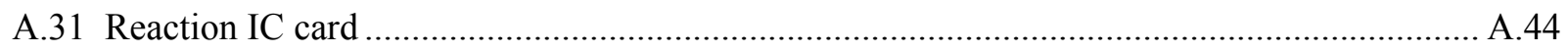

A.31.1 Reaction IC Card Example.................................................................................. A.45

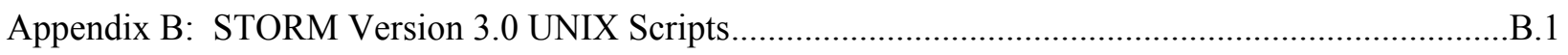

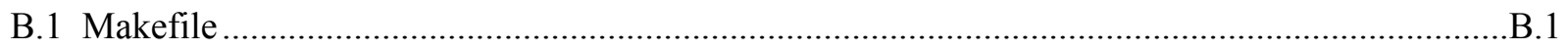

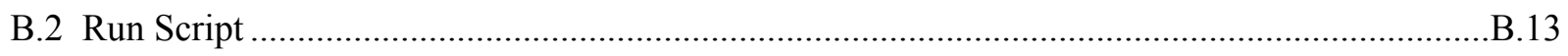

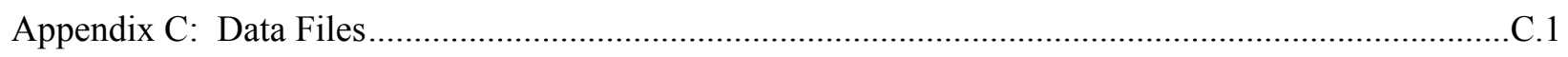

C.1 Input File for Aqueous Phase Advection and Diffusion Test Case ...........................................1

C.2 STORM Input Data File for Gas Phase Advection/Diffusion Test Case ......................................6

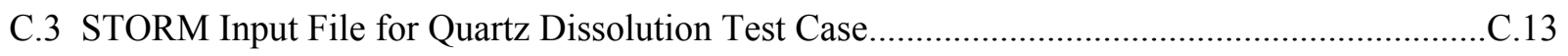

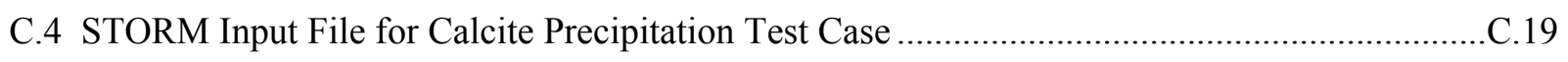

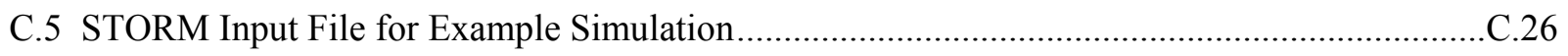




\section{Figures}

3.1 X-Z Coordinate Plane for Cartesian Systems ............................................................................. 3.3

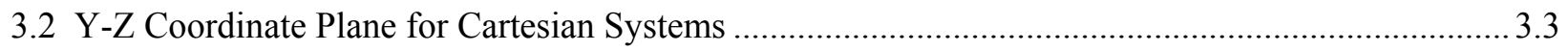

3.3 X-Z Cartesian Coordinate Plane for West Boundary .................................................................2.27

3.4 X-Y Cartesian Coordinate Plane for West Boundary …..............................................................2 3.27

3.5 Pattern of Communication Between Parallel Processes.............................................................. 3.31

8.1 Comparison of STORM Results Using Fully Coupled or Operator Split Solution Modes............... 8.2

8.2 Results of Gas Phase Diffusion and Advection Test Cases ......................................................... 8.3

8.3 Aqueous Silica Concentration as Quartz Dissolves to Equilibrium in Deionized Water .................8.5

8.4 Total $\mathrm{C}$ and $\mathrm{Ca}$ Aqueous Concentrations as Calcite Precipitates from Supersaturated Solution......... 8.7

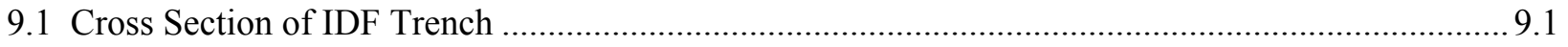

9.2 Material Zonation for WTP Glass Waste Form Release Simulation ............................................. 9.2

9.3 Simulated Steady-State Water Content and Flow Streamlines in Subsurface Disposal Facility ........9.5

9.4 Simulated Waste Glass Dissolution Rate at 10,000 yr............................................................ 9.5

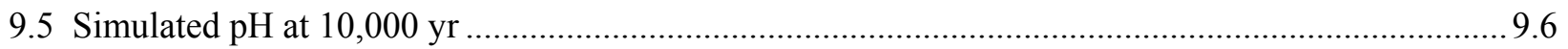

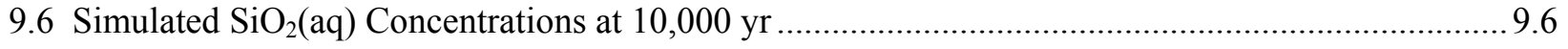

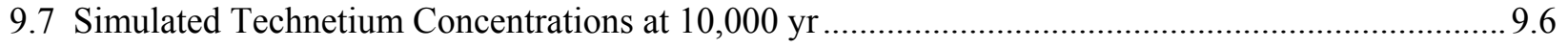

9.8 Technetium Flux Across Lower Model Boundary Relative to Initial Amount of Technetium

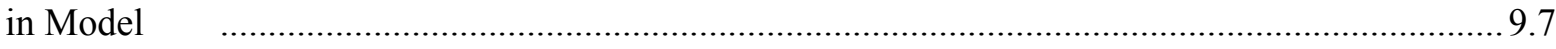

9.9. Scale-up of Parallel STORM on Multiple, Parallel Processors ......................................................... 9.8 


\section{Tables}

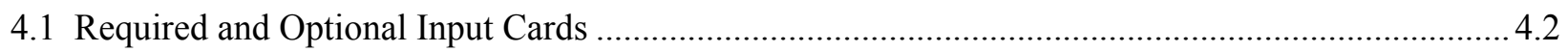

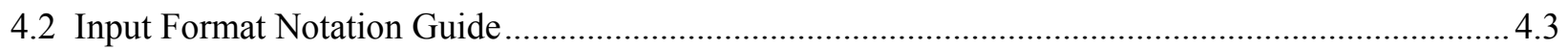

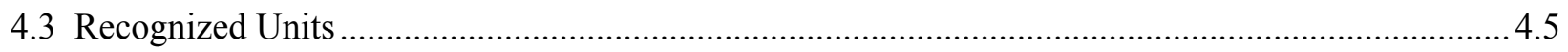

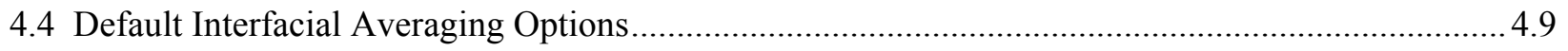

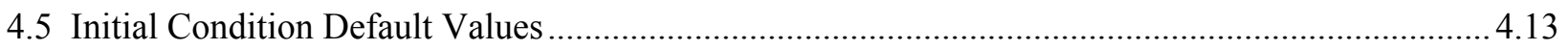

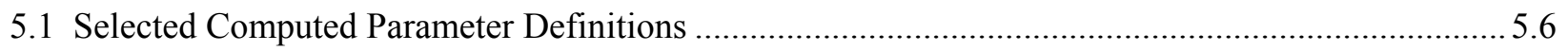

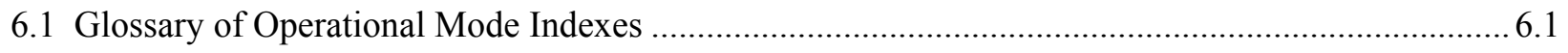

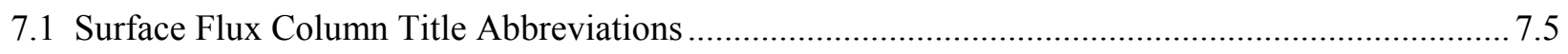

8.1 Simulation Parameters for Aqueous Phase Advection and Diffusion............................................. 8.1

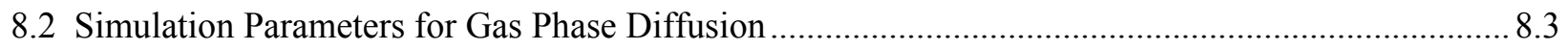

8.3 Aqueous Species, Equilibrium Reactions and Constants Related to Calcite Dissolution.................. 8.6

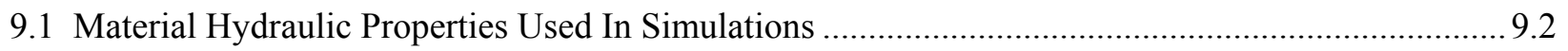

9.2 Secondary Mineral Reaction Network for LAWABP1 Glass...................................................... 9.4

9.3 Equilibrium Reactions Considered in WTP Glass Simulation with Equilibrium Coefficients...........9.4 


\subsection{Introduction}

The Hanford Site, in southeastern Washington State, has been used extensively to produce nuclear materials for the nation's strategic defense arsenal by the U.S. Department of Energy (DOE) and its predecessors, the U.S. Atomic Energy Commission (AEC) and the U.S. Energy Research and Development Administration (ERDA). Over the years, a large inventory of radioactive and mixed waste has accumulated in 177 buried single- and double shell tanks. Liquid waste recovered from the tanks will be pretreated to separate the low-activity fraction from the high-level and transuranic wastes (Ecology 1996). Vitrification is the leading option for immobilization of these wastes and is expected to produce approximately 550,000 metric tons of low-activity waste (LAW) glass. This total tonnage, based on nominal $\mathrm{Na}_{2} \mathrm{O}$ oxide loading of 20 percent by weight, is destined for disposal in a near-surface facility.

Before disposal of the immobilized waste can proceed, DOE must approve a performance assessment, a document that describes the impacts, if any, of the disposal facility on public health and environmental resources. Results from a recent performance assessment (Mann et al. 2001) lead to the conclusion that release rates of radionuclides from the glass waste form by reaction with water determines the effects of the disposal action more than any other independent parameters. Consequently, a sound scientific basis

for determining the long-term release rates of radionuclides from these glasses must be developed before this disposal action is accepted by regulatory agencies, stakeholders, and the public.

The rate of glass corrosion depends on the temperature and the chemical composition of the water contacting it. Because glass dissolution itself changes the $\mathrm{pH}$ and solution composition, water percolating through a disposal system has neither a constant nor a uniform composition. Consequently, the glass corrosion rate in the disposal system also varies as a function of time and space. Over the last several years, DOE has supported the development of a numerical simulator at Pacific Northwest National Laboratory (PNNL). This simulator can compute time and spatial variations in the chemical environment of unsaturated disposal systems in response to the corrosion of waste forms as well as other physical and chemical processes (McGrail and Bacon 1998). This report describes the latest accomplishments in the development of this computational tool, Subsurface Transport over Reactive Multiphases (STORM), Version 3.0, a parallel, coupled non-isothermal multiphase flow and reactive transport simulator. It was developed by coupling STOMP, a non-isothermal multiphase flow simulator (White and Oostrom 1996), with AREST-CT Version 1.1, a reactive transport and porous medium alteration simulator (Chen et al. 1995, 1997). The general approach was coupling the two codes by making major modifications to AREST-CT subroutines and modifying STOMP routines as little as possible.

The underlying mathematics in STORM is contained in a set of coupled, nonlinear, partial differential equations. They describe the rate of change of the solute concentrations of pore water in a variably saturated, non-isothermal porous medium and the alteration of waste forms, packaging materials, backfill, and host rocks. The detailed mathematics is discussed elsewhere (Chen et al. 1996; White and Oostrom 1996). STORM capabilities include:

- kinetic dissolution of waste forms

- kinetic dissolution of host rocks

- kinetic precipitation and dissolution of secondary phases 
- aqueous equilibrium speciation

- gas-aqueous equilibria

- redox reactions

- two-phase flow (water and air)

- dynamic hydraulic properties.

Other physical and chemical features of the code are:

- 1-D (vertical Z-axis) or 2-D (X-Z Plane) solution domains

- general interface to take user specified chemistry and hydraulic properties

- non-isothermal multiphase flow and chemistry

- ionic strength correction according to modified B-dot equation

- spatially varying distribution of minerals in solid phase

- effective reaction surface varies with dissolution and precipitation of solids.

The primary output of STORM, as a function of time and space, consists of the following:

- concentrations of aqueous species

- concentrations of gaseous species

- release flux of aqueous species and chemical components

- $\mathrm{pH}$ and $\mathrm{Eh}$

- radii, surface areas, and volume fractions of solids

- dissolution/precipitation rates of solids

- production/consumption rates of aqueous species, including water

- temperature

- porosity and permeability

- aqueous phase saturations, velocities

- gas phase saturations, velocities.

Section 2 of this document describes the equations that govern STORM, and Section 3 describes the numerical solution methods. Section 4 contains information on the input file structure, and Section 5 presents details of the parameter files. Compilation and execution are described in Section 6 and generation of output files in Section 7. Section 8 contains model verification, section 9 performs an example simulation, and Section 10 contains cited references. Supporting documentation may be found in the appendixes. 


\subsection{Governing Equations}

\subsection{Multiphase Flow and Heat Transfer}

In broad terms, the STORM simulator solves coupled conservation equations for component mass and energy that describe subsurface flow over multiple phases through variably saturated geologic media. The resulting flow fields are used to sequentially solve conservation equations for reactive aqueous phase transport through variably saturated geologic media. These conservation equations for component mass, energy, and solute mass are partial differential equations. They mathematically describe flow and transport through porous media and are collectively entitled "the governing equations." The STORM simulator has capabilities for modeling subsurface flow over two distinct phases: aqueous and gas. The aqueous phase primarily comprises liquid water with small quantities of dissolved air. The gas phase comprises variable amounts of air and water vapor.

It is possible to compile three modes of STORM: STORM1 (unsaturated flow), STORM2 (2-phase flow), and STORM3 (2-phase flow and heat transfer). STORM1 provides the fastest execution time if gas-phase flow and heat transfer are major considerations.

\subsubsection{Water Mass Conservation Equation}

The water mass conservation equation, shown in Equation (2.1), equates the time rate of change of water mass within a control volume with the flux of water mass crossing the control volume surface. In the STORM simulator, water exists in the diffusive pore space as liquid water in the aqueous phase and as water vapor in the gas phase. Phase partitioning of water mass is computed assuming equilibrium conditions, implying the time scale for establishing thermodynamic equilibrium is significantly shorter than for component transport. Water transport occurs by advection through the aqueous and gas phases and also by diffusion-dispersion through the gas phase. Following the low solubility assumption for dissolved air in the aqueous phase, air diffusion-dispersion through the aqueous phase is neglected. Flow of fluid phases is computed from Darcy's law. Transport of phase components is computed from Fick's law:

$$
\frac{\partial}{\partial t}\left[\sum_{\gamma=\ell, g}\left(\phi_{D} \omega_{\gamma}^{w} \rho_{\gamma} s_{\gamma}\right)\right]=-\sum_{\gamma=\ell, g}\left(\nabla \mathbf{F}_{\gamma}^{w}+\nabla \mathbf{J}_{\gamma}^{w}\right)+\dot{m}^{w}
$$

where

$$
\begin{gathered}
\mathbf{F}_{\gamma}^{w}=-\frac{\omega_{\gamma}^{w} \rho_{\gamma} k_{r \gamma} \mathbf{k}}{\mu_{\gamma}}\left(\nabla P_{\gamma}+\rho_{\gamma} g \mathbf{z}_{g}\right) \text { for } \gamma=\ell, g \\
\mathbf{J}_{\gamma}^{w}=-\tau_{\gamma} \phi_{D} \rho_{\gamma} s_{\gamma} \frac{M^{w}}{M_{\gamma}} D_{\gamma}^{w} \nabla \chi_{\gamma}^{w} \text { for } \gamma=\ell, g
\end{gathered}
$$


where

$$
\begin{aligned}
\phi_{D} & =\text { diffusive porosity } \\
\omega_{\gamma}^{j} & =\text { mole fraction of component } \mathrm{j} \text { in phase } \gamma \\
\rho_{\gamma} & =\text { phase density for phase } \gamma, \mathrm{kg} \mathrm{m}^{-3} \\
\mathbf{s}_{\gamma} & =\text { phase saturation for phase } \gamma \\
\mathbf{F}_{\gamma}^{j} & =\text { advective flux of component } \mathrm{j} \text { in phase } \gamma, \mathrm{kg} \mathrm{m}^{-2} \mathrm{~s}^{-1} \\
\dot{m}^{w} & =\text { mass source rate of component } \mathrm{j}, \mathrm{kg} \mathrm{m}^{-3} \mathrm{~s}^{-1} \\
\mathbf{J}_{\gamma}^{j} & =\text { diffusive-dispersive flux of component } \mathrm{j} \text { for phase } \gamma, \mathrm{kg} \mathrm{m}^{-2} \mathrm{~s}^{-1} \\
\mathbf{k} & =\text { intrinsic permeability tensor, } \mathrm{m}^{2} \\
k_{r \gamma} & =\text { fluid relative permeability of phase } \gamma \\
P_{\gamma} & =\text { pressure of phase } \gamma, \text { Pa } \\
\mathbf{z}_{g} & =\text { unit gravitational direction vector } \\
g & =\text { acceleration of gravity, } \mathrm{m} \mathrm{s}^{-2} \\
\mu_{\gamma} & =\text { kinematic viscosity of phase } \gamma, \text { Pa s } \\
\tau_{\gamma} & =\text { phase tortuosity for phase } \gamma \\
\mathrm{M}_{\gamma} & =\text { molecular weight of phase } \gamma, \mathrm{kg} \text { mol }^{-1} \\
D_{\gamma}^{j} & =\text { diffusion coefficient of component } \mathrm{j} \text { for phase } \gamma, \mathrm{m}^{2} \mathrm{~s}^{-1} \\
\chi_{\gamma}^{j} & =\text { mole fraction of component } \mathrm{j} \text { in phase } \gamma \\
a & =\text { air component } \\
w & =\text { water component } \\
g & =\text { gas phase } \\
\ell & =\text { aqueous phase } \\
t & =\text { time, } \mathrm{s} .
\end{aligned}
$$

\subsubsection{Air Mass Conservation Equation}

Similar to the water mass conservation equation, the air mass conservation equation, shown in Equation (2.4), equates the time rate of change of the air mass within a control volume with the flux of air mass crossing the control volume surface. In the STORM simulator, air exists in the diffusive pore space as a component of the gas phase and is dissolved in the aqueous phase. Phase partitioning of air mass is computed assuming equilibrium conditions: this assumption implies the time scale for thermodynamic equilibrium in geologic media is significantly shorter than that for component transport. Air transport occurs by advection and diffusion dispersion through the aqueous and gas phases. Flow of fluid phases is computed from Darcy's law. Transport of phase components is computed from Fick's law.

$$
\frac{\partial}{\partial t}\left[\sum_{\gamma=\ell, g}\left(\phi_{D} \omega_{\gamma}^{a} \rho_{\gamma} s_{\gamma}\right)\right]=-\sum_{\gamma=\ell, g}\left(\nabla \mathbf{F}_{\gamma}^{a}+\nabla \mathbf{J}_{\gamma}^{a}\right)+\dot{m}^{a}
$$


where

$$
\begin{gathered}
\mathbf{F}_{\gamma}^{a}=-\frac{\omega_{\gamma}^{a} \rho_{\gamma} k_{r \gamma} \mathbf{k}}{\mu_{\gamma}}\left(\nabla P_{\gamma}+\rho_{\gamma} g \mathbf{z}_{g}\right) \text { for } \gamma=\ell, g \\
\mathbf{J}_{\gamma}^{a}=-\tau_{\gamma} \phi_{D} \rho_{\gamma} s_{\gamma} \frac{M^{a}}{M_{\gamma}} D_{\gamma}^{a} \nabla \chi_{\gamma}^{a} \text { for } \gamma=\ell, g
\end{gathered}
$$

\subsubsection{Energy Conservation Equation}

The energy conservation equation, shown in Equation (2.7), equates the time rate of change of energy within a control volume with the flux of energy crossing the control volume surface. In the STORM simulator, thermal energy is partitioned according to thermal equilibrium conditions among the fluid and solid phases. The thermal capacitance of unconnected pore space, represented by the difference between the total and diffusive porosity, is computed as it is filled with liquid water. Heat transfer occurs by advection of phase mass, diffusion of component mass, and thermal diffusion through the fluid and solid phases. Heat transfer by hydraulic dispersion of flowing fluid phases is neglected. Enhanced vapor transport is incorporated through enhancement factors for component diffusion through the gas phase. Energy associated with component mass sources is included as internal heat generation sources. Reference states for enthalpy and internal energy are component dependent. Latent heat transport is considered through vapor transport using the gas phase and equilibrium thermodynamics.

$$
\begin{aligned}
& \frac{\partial}{\partial t}\left[\sum_{\gamma=\ell, g}\left(\phi_{D} \rho_{\gamma} s_{\gamma} u_{\gamma}\right)+\left(1-\phi_{T}\right) \rho_{s} u_{s}+\left(\phi_{T}-\phi_{D}\right) \rho_{\ell} u_{\ell}\right]= \\
& -\sum_{\gamma=\ell, g}\left(\nabla\left(\rho_{\gamma} h_{\gamma} \mathbf{q}_{\gamma}\right)+\sum_{j=w, a}\left(\nabla h^{j} \mathbf{J}_{\gamma}^{j}\right)\right)+\nabla\left(\mathbf{k}_{e} \nabla T\right)+\sum_{j=w, a}\left(h^{j} \dot{m}^{j}\right)+\dot{Q}
\end{aligned}
$$

where the Darcy velocity is given by

$$
\mathbf{q}_{\gamma}=-\frac{k_{r \gamma} \mathbf{k}}{\mu_{\gamma}}\left(\nabla P_{\gamma}+\rho_{\gamma} g \mathbf{z}_{g}\right) \text { for } \gamma=\ell, g
$$

and the diffusive flux by

$$
\mathbf{J}_{\gamma}^{j}=-\tau_{\gamma} \phi_{D} \rho_{\gamma} s_{\gamma} \frac{M^{j}}{M_{\gamma}} D_{\gamma}^{j} \nabla \chi_{\gamma}^{j} \text { for } \gamma=\ell, g \text { and } j=w, a
$$

where

$$
\begin{aligned}
& \phi_{T}=\text { total porosity } \\
& h_{j}=\text { enthalpy of component } \mathrm{j}, \mathrm{J} \mathrm{kg}^{-1}
\end{aligned}
$$




$$
\begin{aligned}
h_{\gamma} & =\text { enthalpy of phase } \gamma, \mathrm{J} \mathrm{kg}^{-1} \\
\mathbf{k}_{e} & =\text { equivalent thermal conductivity tensor, } \mathrm{W} \mathrm{m}^{-1} \mathrm{~K}^{-1} \\
T & =\text { temperature, } \mathrm{K} \\
\dot{Q} & =\text { thermal energy source, } \mathrm{W} \\
\mu_{\gamma} & =\text { kinematic viscosity of phase } \gamma, \mathrm{Pa} \mathrm{s} \\
\mu_{\gamma} & =\text { internal energy of phase } \gamma, \mathrm{J} \mathrm{kg}^{-1} .
\end{aligned}
$$

\subsection{Solute Transport}

\subsubsection{Solute Mass Conservation Equation}

The solute mass conservation equation shown in Equation (2.10) equates the time rate for change of solute mass within a control volume with the flux of solute mass crossing the control volume surface. The solute mass conservation equation is solved when an Operator Split solution scheme is used. In the STORM simulator, solute mass is partitioned among the fluid and solid phases assuming thermodynamic and geochemical equilibrium conditions. This assumption implies the time scales for thermodynamic and geochemical equilibrium are short compared to those for solute transport. For geologic media, this assumption is generally appropriate. Solute transport occurs by advection and diffusion-dispersion through the aqueous phase and gas phase. Radioactive decay of solutes is computed using first-order decay kinetics. The solute mass conservation equation is given by:

$$
\frac{\partial C}{\partial t}=-\sum_{\gamma=l, g}\left(\nabla\left[C_{\gamma} q_{\gamma}\right]\right)+\dot{m}^{C}-\dot{R}^{C} C+\sum_{\gamma=l, g}\left(\nabla\left[\left(\tau_{\gamma} s_{\gamma} \phi_{D} D_{\gamma}^{C}+s_{\gamma} \phi_{D} \mathbf{D}_{h \gamma}\right) \nabla C_{\gamma}\right]\right)
$$

where

$C=$ mass of solute per unit volume of porous media, $\mathrm{m}^{-3}$

$C_{\gamma}=$ mass of solute per unit volume of either liquid or gas, $\mathrm{m}^{-3}$

$D_{\gamma}^{C}=$ solute diffusion coefficient for phase $\gamma, \mathrm{m}^{2} \mathrm{~s}^{-1}$

$\mathbf{D}_{h \gamma}=$ hydraulic dispersion tensor for phase $\gamma, \mathrm{m}^{2} \mathrm{~s}^{-1}$

$\dot{m}_{C}=$ solute source rate, $\mathrm{s}^{-1}$

$\dot{R}^{C}=$ solute decay rate constant, $\mathrm{s}^{-1}$.

\subsubsection{Aqueous Species Mass Conservation Equation}

The aqueous species mass conservation equation is written as: 


$$
\frac{\partial \phi_{D} \rho_{\ell} s_{\ell} C_{i}^{\ell}}{\partial t}=-\nabla \cdot F_{i}^{\ell}+S_{i}^{r}
$$

where $C_{i}^{\ell}$ is the concentration of species $i$ in the aqueous phase (denoted by $\ell$ ) in mol $\mathrm{kg}^{-1}, F_{i}^{\ell}$ is the solute flux of species $i$ in the aqueous phase, $S_{i}^{r}$ is the source/sink term of species $i$ due to chemical reactions in mol m $\mathrm{m}^{-3}$. The aqueous species mass conservation equation is solved when a Fully Coupled solution scheme is used.

If it is assumed that the phenomenon of hydrodynamic dispersion can be represented by a Fickian-type law, the total solute flux due to advection, dispersion, and diffusion is given by

$$
\mathbf{F}_{i}^{S}=\phi_{D} \rho_{\ell} s_{\ell}\left(C_{i} \mathbf{V}_{\ell}-\mathbf{D}\left(s_{\ell}\right) \cdot \nabla C_{i}\right)
$$

where $\mathbf{D}$ is the hydrodynamic dispersion tensor of species $i$ in the aqueous phase, $\mathrm{m}^{2} \mathrm{~s}^{-1}$.

The flow fields resulting from the air, water, and energy conservation equations are coupled with the solute mass conservation equations via transient changes in water saturation and average linear pore water velocity. This velocity is related to the Darcy velocity by

$$
\mathbf{V}_{\ell}=\frac{\mathbf{q}_{\ell}}{\phi_{D} s_{\ell}}
$$

\subsubsection{Coupled Aqueous and Gaseous Species Mass Conservation Equation}

The mass conservation law for a particular species can be written as the sum of transport in the gas and aqueous phases:

$$
\frac{\partial}{\partial t} \rho_{\ell} \phi_{D}\left(s_{g} C_{i}^{g}+s_{l} C_{i}^{\ell}\right)=-\nabla \bullet F_{i}^{S}+S_{i}^{r}
$$

where $F_{i}^{S}$ is the total flux of species $i$ in both the gas and aqueous phases. by

The total flux due to advection, dispersion, and diffusion in both the aqueous and gas phases is given

$$
F_{i}^{S}=\phi_{D} s_{g}\left(\mathbf{V}_{g} C_{i}^{g}-\mathbf{D}_{i}^{g} \cdot \nabla C_{i}^{g}\right)+\phi_{D} s_{\ell}\left(\mathbf{V}_{\ell} C_{i}^{\ell}-\mathbf{D}_{i}^{\ell} \cdot \nabla C_{i}^{\ell}\right)
$$


where $V_{g}$ is the pore-gas velocity and $\mathbf{D}_{i}^{g}$ is the hydrodynamic dispersion tensor for species $i$ in the gas phase. The coupled aqueous and gaseous species mass conservation equation is solved when a Fully Coupled solution scheme is used.

Assuming equilibrium between the gaseous phase and aqueous phase, the concentration of the dissolved gas component in the aqueous phase can be expressed as a linear function of the concentration (or mole fraction or partial pressure) of that component in the gaseous phase through Henry's constant

$$
C_{i}^{\ell}=K_{H} p_{i}^{g}=K_{H}^{\prime} C_{i}^{g}
$$

where

$$
\begin{aligned}
C_{i}^{\ell} & =\text { aqueous phase concentration of the dissolved gas component } \\
p_{g} & =\text { partial pressure of the component in the gas phase } \\
C_{i}^{g} & =\text { gas phase concentration of the component } \\
K_{H} & =\text { Henry's law constants expressed as a function of partial pressure } \\
K_{H}^{\prime} & =\text { Henry's law constants expressed as a function of mole fraction. }
\end{aligned}
$$

Substituting Equations (2.15) and (2.16) into (2.14) gives the mass conservation law in terms of the aqueous phase concentration of each species:

$$
\begin{gathered}
\frac{\partial}{\partial t}\left[\phi_{D} \rho_{\ell}\left(s_{g} K_{H}^{\prime-1}+s_{\ell}\right) C_{i}^{\ell}\right]=S_{i}^{r}+ \\
\nabla \bullet\left[-\phi_{D} \rho_{\ell}\left(s_{g} \mathbf{V}_{g} K_{H}^{\prime-1}+s_{\ell} \mathbf{V}_{\ell}\right) C_{i}^{\ell}+\phi_{D} \rho_{\ell}\left(s_{g} \mathbf{D}_{i}^{g} K_{H}^{\prime-1}+s_{\ell} \mathbf{D}_{i}^{\ell}\right) \cdot \nabla C_{i}^{\ell}\right]
\end{gathered}
$$

A series of transformations is applied to Equation (2.17) to eliminate terms associated with equilibrium chemical reactions. Each aqueous species is defined as a reactive species, a non-reactive species, or an equilibrium species. Because of the transformations applied, equilibrium species must be transported at the same rate as the linearly related reactive species. A different Henry's Law constant and dispersion coefficient is allowed only for each reactive aqueous species that is in equilibrium with a gas species.

\subsection{Geochemical Reactions}

A chemical reaction generally can be written as

$$
v_{m j} B_{m}+\sum_{i=1}^{N_{s}} v_{i j} A_{i}=0
$$

where

$$
B_{m}=\operatorname{solid} m
$$




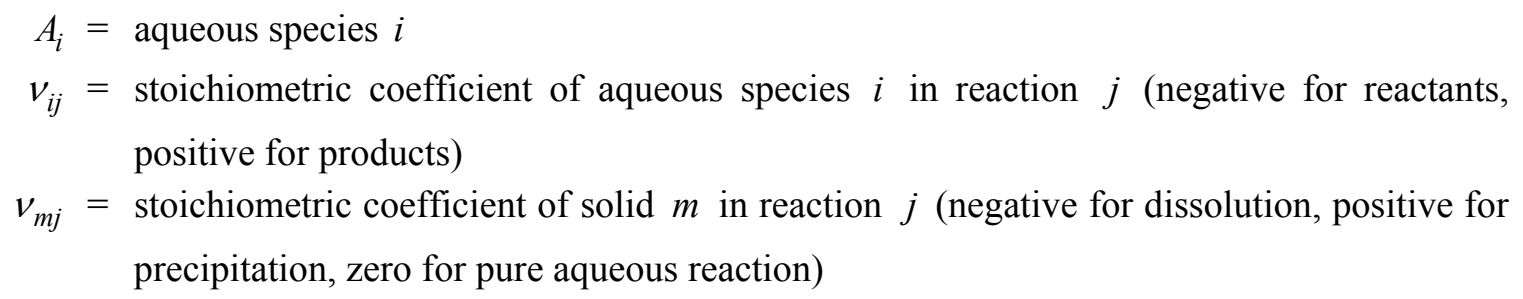

If the rate of the reaction in unit bulk volume is shown as $W_{j}$ (in general a nonlinear function of the aqueous species concentrations), the rate of aqueous species $i$ in that reaction is $v_{i j} W_{j}$. (This nonlinear function is defined in Section 2.3.2.) The source/sink term due to reactions in Equation (2.14) is then

$$
S_{i}^{r}=\sum_{j=1}^{N_{r}} v_{i j} W_{j}
$$

where $N_{r}$ is the number of reactions.

\subsubsection{Equilibrium and Kinetic Reactions}

Geochemical models usually assume some reactions to be in equilibrium. This assumption is often justified for some reactions, especially those involving only aqueous species. However, solid dissolution/precipitation reactions can be quite slow and should be treated as kinetic. Furthermore, the equilibrium approach cannot capture some metastable solid phases, such as amorphous solids, that in many cases are intermediate products of reaction. For this reason, STORM has been designed to treat both equilibrium and kinetic reactions separately. To this end, the source/sink term is split, due to chemical reactions, into equilibrium and kinetic parts.

$$
S_{i}^{r}=\sum_{j=1}^{N_{e}} v_{i j} W_{j}^{e}+\sum_{j=1}^{N_{k}} v_{i j} W_{j}^{k}
$$

where

$$
\begin{aligned}
W_{j}^{e} & =\text { the rate of equilibrium reaction } j\left(\mathrm{~mol} \mathrm{~m}^{-3} \mathrm{~s}^{-1}\right) \\
W_{j}^{k} & =\text { the rate of kinetic reaction } j\left(\mathrm{~mol} \mathrm{~m}^{-3} \mathrm{~s}^{-1}\right) \\
N_{e} & =\text { the number of equilibrium reactions } \\
N_{k} & =\text { the number of kinetic reactions } \\
N_{r} & =N_{e}+N_{k} .
\end{aligned}
$$

Equilibrium reactions are no zero-rate reactions but have high reaction rates and reach equilibrium quickly when transport, other reactions, or changes in physical-chemical conditions disturb it. Specifically, if the rate of a reaction is much greater than the characteristic time of the problem being solved, it should be classified as equilibrium; otherwise it should be classified as kinetic. 


\subsubsection{Rate Law of Kinetic Reactions}

For any kinetic reaction, assuming its rate law is of the mass-action type, two equivalent ways can define the reaction rate: one is to use the forward rate and backward rate; the other is to use the forward rate and the equilibrium constant. In STORM, the latter approach is used. Thus,

$$
W_{j}^{k}=A_{m(j)} k_{j}\left[\prod_{v_{i j}<0}\left(\gamma_{i} c_{i}\right)^{\frac{\left|v_{i j}\right|}{f_{j}}}-\prod_{v_{i j}>0}\left(\gamma_{i} c_{i}\right)^{\frac{v_{i j}}{f_{j}}} /\left(K_{j}^{e q}\right)^{1 / f_{j}}\right]
$$

where

$$
\begin{aligned}
& \mathrm{A}_{\mathrm{m}(\mathrm{j})}=\mathrm{a} \text { factor that for aqueous reactions equals } 1 \text {, and for surface-limited reactions equals } \\
& \text { the specific surface area of mineral } m \text { in unit bulk volume of porous media, } \mathrm{m}^{2} \mathrm{~m}^{-3} \\
& k_{j}=\text { the rate constant of reaction } j, \mathrm{~mol} \mathrm{~m}^{-2} \mathrm{~s}^{-1} \\
& K_{j}^{e q} \quad=\text { the equilibrium constant of reaction } j \\
& \gamma_{\mathrm{i}}=\text { the activity coefficient of species } i \\
& f_{j} \quad=\text { the factor of the reaction order. }
\end{aligned}
$$

Several formulas, such as the Davies equation (Davies 1962), the B-dot equation (Helgeson 1969), and Pitzer's model (Pitzer 1973), can be used to calculate activity coefficients. The B-dot equation, with modifications for neutral species (Wolery 1992), is computationally economic and stable for problems of moderately high salinity. Although Pitzer's model can handle high ionic strength, the lack of constants for all but a few aqueous species limits its applicability. Thus, the B-dot equation is used to calculate activity coefficients in STORM.

\subsubsection{Solid Reactions}

In the subsurface, chemical reactions change not only the concentration of solutes but also the properties of the porous media. Changes in the volume fraction and surface area of solids, porosity and permeability result in changes in the fluid flow field, solute transport, and chemical reactions. Interactions may take place through several paths and in some cases be very strong. Ignoring the interactions will not only decrease the accuracy of the simulations but, in many cases, also will yield even qualitatively incorrect predictions. To fully account for the interactions between the fluid phases and the porous media, it is necessary to consider dynamic changes in the properties of porous media as a result of mass redistribution from chemical reactions. The first step is to introduce variables that describe porous media texture.

STORM uses two primary variables to describe a mineral: the volume fraction $V_{m}$ and one or more sizes of variables (depending on the shape of the mineral grains). The volume fractions of minerals and the porosity of the porous medium obey the relation

$$
\sum_{m=1}^{N_{m}} V_{m}+\phi_{T}=1
$$


From the volume fraction and specific surface area $A_{m}$ (surface area of mineral $\mathrm{m}$ in unit bulk volume of porous media), the number density $n_{m}$ (number of grains of mineral $m$ in unit bulk volume of porous media) is derived and the size variables are determined when the shape of the grains is specified. For spherical grains, the size variable is the radius $R_{m}$, so that

$$
R_{m}=\frac{3 V_{m}}{A_{m}}
$$

and

$$
n_{m}=\frac{A_{m}}{4 \pi R_{m}^{2}}
$$

By assuming that a dissolution/precipitation reaction is a surface-limited kinetic reaction and observing the rate law of Equation (2.21), the change rate of the volume fraction is

$$
\frac{\partial V_{m}}{\partial t}=4 \pi R_{m}^{2} n_{m} \frac{\partial R_{m}}{\partial t}
$$

For general cases of more than one dissolution/precipitation reaction occurring for one mineral, the rate of change of the mineral radius is

$$
\frac{\partial R_{m}}{\partial t}=\sum_{j=1}^{N_{k}} \frac{k_{j}}{\rho_{m}}\left[\prod_{v_{i j}<0}\left(\gamma_{i} c_{i}\right)^{\frac{\left|v_{i j}\right|}{f_{j}}}-\prod_{v_{i j}>0}\left(\gamma_{i} c_{i}\right)^{\frac{v_{i j}}{f_{j}}} /\left(K_{j}^{e q}\right)^{1 / f_{j}}\right]
$$

where $\rho_{m}$ is the molar density of mineral $m\left(\mathrm{~mol} \mathrm{~m}^{-3}\right)$, and the summation is over all kinetic reactions pertaining to mineral $m$.

Substituting Equation (2.24) into Equation (2.21) gives

$$
W_{j}^{k}=4 \pi R_{m(j)}^{2} n_{m(j)} k_{j}\left[\prod_{v_{i j}<0}\left(\gamma_{i} c_{i}\right)^{\frac{\left|v_{i j}\right|}{f_{j}}}-\prod_{v_{i j}>0}\left(\gamma_{i} c_{i}\right)^{\frac{v_{i j}}{f_{j}}} /\left(K_{j}^{e q}\right)^{1 / f_{j}}\right]
$$

which serves as a bridge between the aqueous phase and solid phases so that the whole system is mass conservative. 


\subsection{Coupling Between Fluid Flow and Geochemical Reactions}

\subsubsection{Porosity Changes Coupled with Multiphase Flow}

Porosity changes due to mineral dissolution and precipitation could have a significant effect on the flow regime surrounding the disposal facility. Therefore, porosity changes calculated by the STORM texture solver are coupled with the multiphase flow solver. Dissolution and precipitation reactions can change the volume fractions of solids as a function of time, thus changing the porosity, as described in Section 2.3.3. Furthermore, intrinsic permeability varies as a function of porosity. Two options for modeling this relationship are available in STORM. One is the Fair and Hatch (1933) formula, developed from dimensional considerations and verified experimentally:

$$
k=\frac{1}{\beta}\left[\frac{\left(1-\phi_{T}\right)^{2}}{\phi_{T}^{3}}\left(\frac{\alpha}{100} \sum_{(m)} \frac{P_{m}}{d_{m}}\right)^{2}\right]^{-1}
$$

where

$k=$ intrinsic permeability, $\mathrm{cm}^{2}$

$\beta=$ packing factor, found experimentally to be about 5

$\alpha=$ sand shape factor, varying from 6.0 for spherical grains to 7.7 for angular ones

$P_{m}=$ percentage of sand held between adjacent sieves

$d_{m}=$ geometric mean diameter of adjacent sieves.

The second option is the Kozeny-Carmen equation that was obtained from a theoretical derivation of Darcy's law and includes numerical coefficients that must be determined empirically:

$$
k=C_{0} \frac{\phi_{T}^{3}}{\left(1-\phi_{T}\right)^{2} M_{S}^{2}},
$$

where

$M_{S}=$ specific surface area of the porous matrix (defined per unit volume of solid)

$C_{0}=$ coefficient for which Carman (1937) suggested the value of $1 / 5$.

\subsubsection{Water Consumption in Chemical Reactions Coupled with Multiphase Flow}

The consumption/production of water during glass dissolution or the precipitation of hydrated minerals may be large enough to affect unsaturated flow in the region of the waste repository. The STORM code couples unsaturated flow with water production/consumption by chemical reactions through the mass source rate of water in Equation (2.1).

$$
\dot{m}^{w}=\sum_{j=1}^{N_{r}} v_{w j} W_{j}^{k} / \Omega
$$


where

$$
\begin{aligned}
& N_{r}=\text { the total number of reactions } \\
& v_{w j}=\text { the stoichiometric coefficient of water in reaction } j \\
& W_{j}^{k}=\text { the rate of reaction per unit bulk volume, given by Equation }(2.21) \\
& \Omega=\text { the number of moles of solvent water comprising a mass of } 1 \mathrm{~kg}(\Omega=55.51) .
\end{aligned}
$$

The water source/sink term due to reactions is added to any other water source/sinks specified in the STORM input file.

\subsubsection{Gas Consumption in Chemical Reactions Coupled with Multiphase Flow}

Consumption of gaseous species, particularly oxygen, may occur at large enough rates to affect the flow of air in the vadose zone. The STORM code couples unsaturated flow with gas production/ consumption by chemical reactions through the mass source rate of air in Equation (2.4):

$$
\dot{m}^{a}=\sum_{j=1}^{N_{r}} v_{g j} W_{j}^{k} M_{j}
$$

where

$$
\begin{aligned}
& N_{r}=\text { the total number of reactions } \\
& v_{g j}=\text { the stoichiometric coefficient of the gaseous species in reaction } j \\
& W_{j}^{k}=\text { the rate of reaction per unit bulk volume, given by Equation (2.21) } \\
& \mathrm{M}_{\mathrm{j}} \quad=\text { is the molecular weight of the gaseous species. }
\end{aligned}
$$

The gas source/sink term due to reactions is added to any other gas source/sinks specified in the STORM input file. 


\subsection{Numerical Solution Methods}

The STORM simulator solves the governing conservation equations and constitutive functions using numerical techniques for nonlinear systems. This section documents the transformation of the governingconservation equations from partial-differential form to algebraic form, algebraic expression of boundary conditions, linearization of the conservation equations and constitutive functions, and solution of linear systems.

The governing-conservation equations for water, air, and energy conservation are discretized to algebraic form following the integrated finite-difference method of Patankar (1980). This transformation requires the physical domain to be spatially discretized into an orthogonal computational domain comprising non-overlapping volumes (nodes). Each volume can contain a maximum of two neighboring nodes for each dimensional direction. Intrinsic properties are assumed to be uniform over the volume domain and are defined for a node point at the geometric center of the volume. Flux quantities are defined at the geometric center of the surfaces between node volumes and along a direction parallel to the surface normal. Fluxes across node surfaces between neighboring inactive nodes or adjacent to the domain boundary are controlled through boundary conditions. Solution of the governing-conservation equations in time requires discretization of the time domain. The method of Patankar (1980) is implicit using backward Euler time differencing. The expressions that result from discretizing the governing equations are nonlinear algebraic equations.

The STORM simulator uses a Cartesian grid system. Coordinate directions for Cartesian systems follow the right-hand rule from the $\mathrm{x}$ to $\mathrm{y}$ to $\mathrm{z}$ directions. Positive and negative directions along the $\mathrm{x}, \mathrm{y}$, and $\mathrm{z}$ coordinates are referred to as east, west, north, south, top, and bottom, respectively. The gravitational vector can be aligned arbitrarily with respect to the Cartesian directions.

The algebraic equations that include the discretized governing conservation equations and the constitutive functions are nonlinear. Nonlinearities in the soil-moisture retention functions, relative permeability functions, and physical properties near phase transitions are the primary contributors. Conversion of the algebraic equations from nonlinear to linear form follows the iterative NewtonRaphson (Kreyszig 1979) technique for multiple variables. Typically, the technique yields quadratic convergence of the residuals given sufficiently close estimates of the primary unknowns. Each iteration loop requires the solution of a system of linear equations in terms of the equation residuals. Because only orthogonal grid systems are considered, the system of linear equations has a block-banded structure.

The governing conservation equations are discretized following the integrated finite difference of Patankar (1980) that is locally and globally conserving. Mass conservation equations for water and air components are nearly identical in form, and therefore result in similar algebraic forms. The conservation equation for energy differs from the mass conservation equations having diffusive-dispersive and advective components. Discretization of combined diffusive and advective transport requires donor-cell weighting of the transport components, yielding algebraic forms different than those for the mass conservation equations. The conservation equation for solute transport is similar in form to the energy conservation equation, but its discretization uses a different donor-cell weighting scheme, resulting in a separate algebraic form. 


\subsection{Water and Air Mass Conservation Equation}

The water and air mass conservation equations shown in Equations (2.1) and (2.4) are discretized by assuming a piecewise profile to express the variation in primary variables between node points and integrating over the node volume. The accumulation terms (that is, terms on the left side) are integrated over the node volume according to Equation (3.1), where the specific terms for the water and air conservation equations appear as shown in Equations (3.2) and (3.3), respectively. Intrinsic properties for the node volume are represented by properties at the node centroid. Source terms are similarly integrated over the node volume according to Equation (3.4).

$$
\begin{gathered}
\frac{\partial}{\partial t}\left[\int_{\bar{V}} \bar{M}^{j} d \bar{V}\right]=\frac{\partial}{\partial t}\left[\bar{M}^{j} \bar{V}\right] \text { for } j=w, a \\
\frac{\partial}{\partial t}\left[\bar{M}^{w} \bar{V}\right]=\frac{\partial}{\partial t}\left[\bar{V} \sum_{\gamma=l, g}\left(\phi_{D} \omega_{\gamma}^{w} \rho_{\gamma} s_{\gamma}\right)\right] \\
\frac{\partial}{\partial t}\left[\bar{M}^{a} \bar{V}\right]=\frac{\partial}{\partial t}\left[\bar{V} \sum_{\gamma=l, g}\left(\phi_{D} \omega_{\gamma}^{a} \rho_{\gamma} s_{\gamma}\right)\right] \\
\int_{\bar{V}} \dot{m}^{j} d \bar{V}=\dot{m}^{j} \bar{V} \text { for } j=w, a
\end{gathered}
$$

Flux terms are evaluated on the node surfaces and for the mass conservation equations comprise advective and diffusive components. Integration of the flux terms over the node volume proceeds by first converting the volumetric integral of flux over a control volume to a surface integral through Green's theorem (Kreyszig 1979) according to Equation (3.5). Discretizing the control volume surfaces into node surfaces and defining flux directions parallel to the surface normal allows the surface integrals to be converted to summations over all node surfaces according to Equation (3.6). This transformation strictly requires an orthogonal grid system for the flux directions to be aligned with the surface normals. Nonorthogonal systems will yield mass balance errors. Darcy fluxes are discretized in the six coordinated directions using upwind interfacial averaging for the component mass fraction, phase density, and relative permeability and harmonic averaging for the intrinsic permeability and phase viscosity according to Equation (3.6). These default interfacial averaging schemes can be altered by user input. Diffusive fluxes are discretized in the six coordinate directions using harmonic averaging for the combination of terms that compose an effective diffusion coefficient. The user can also alter the default interfacial averaging scheme for each parameter. Geometric variables for a Cartesian coordinate system are shown in Figure 3.1 for the X-Z coordinate plane and Figure 3.2 for the $\mathrm{Y}-\mathrm{Z}$ coordinate plane. 

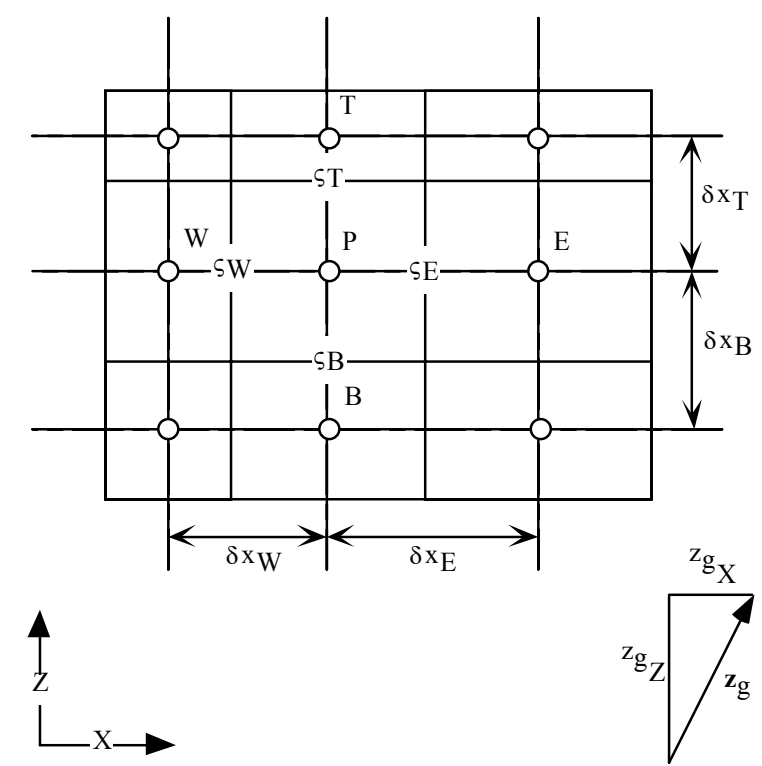

Figure 3.1. $X-Z$ Coordinate Plane for Cartesian Systems
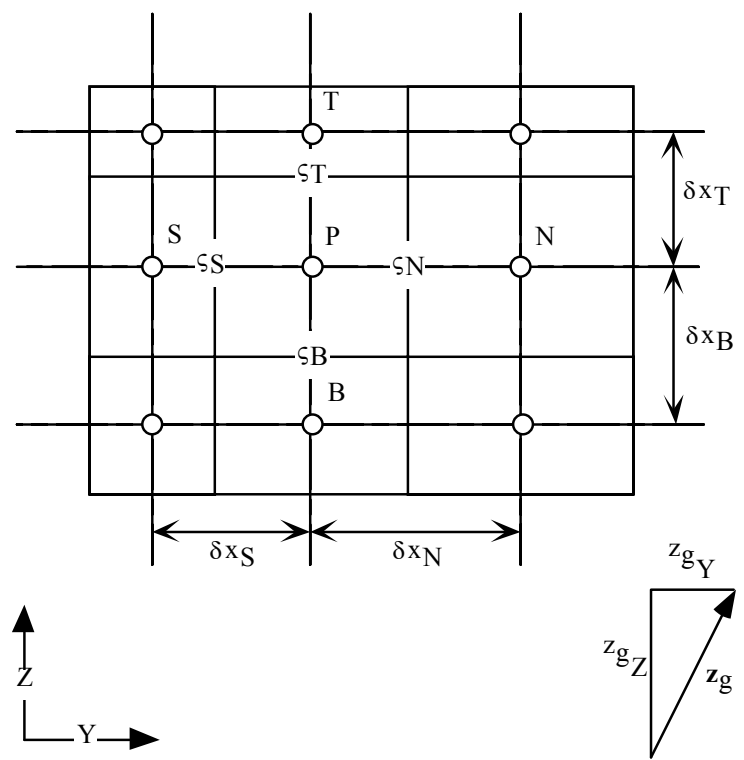

Figure 3.2. $\mathrm{Y}-\mathrm{Z}$ Coordinate Plane for Cartesian Systems

$$
\int_{\bar{V}}\left[\sum_{\gamma=l, g}\left(\nabla \mathbf{F}_{\gamma}^{j}+\nabla \mathbf{J}_{\gamma}^{j}\right) d \bar{V}\right]=\int_{\Gamma}\left[\sum_{\gamma=l, g}\left(\mathbf{F}_{\gamma}^{j}+\mathbf{J}_{\gamma}^{j}\right) \cdot \mathbf{n} d \Gamma\right] \text { for } j=w, a
$$

where

$\int_{\bar{V}}[] d \bar{V}=$ volume integral 


$$
\begin{array}{ll}
\int_{\Gamma}[] d \Gamma=\text { surface integral } \\
\sum_{\gamma=l, g}=\text { summation over aqueous and gas phases } \\
\sum_{j=w, a}=\text { summation over water and air components }
\end{array}
$$

where

$$
\begin{gathered}
\mathbf{F}_{\gamma}^{j}=\frac{\omega_{\gamma}^{j} \rho_{\gamma} k_{r \gamma} \mathbf{k}}{\mu_{\gamma}}\left(\nabla P_{\gamma}+\rho_{\gamma} g \mathbf{z}_{g}\right) \text { for } \gamma=l, g \text { and } j=w, a \\
\mathbf{J}_{\gamma}^{j}=-\tau_{\gamma} \phi_{D} \rho_{\gamma} s_{\gamma} \frac{M^{j}}{M_{\gamma}} D_{\gamma}^{j} \nabla \chi_{\gamma}^{j} \text { for } \gamma=l, g \text { and } j=w, a \\
\int_{\Gamma}\left[\sum_{\gamma=l, g}\left(\mathbf{F}_{\gamma}^{j}+\mathbf{J}_{\gamma}^{j}\right) \cdot \mathbf{n} d \Gamma\right]=\sum_{\varsigma=\mathrm{W}, \mathrm{E}, \mathrm{S}, \mathrm{N}, \mathrm{T}, \mathrm{B}}\left[\sum_{\gamma=1, \mathrm{~g}}\left(\mathrm{~F}_{\gamma \varsigma}^{\mathrm{j}}+J_{\gamma \varsigma}^{\mathrm{j}}\right)\right] \mathrm{A}_{\varsigma} \text { for } j=w, a
\end{gathered}
$$

where $A_{\varsigma}$ is the area of surface $\varsigma$, and where

$$
\begin{gathered}
F_{\gamma \varsigma}^{j}=-\frac{\left\langle\omega_{\gamma}^{j} \rho_{\gamma} k_{r \gamma}\right\rangle_{\varsigma}^{u w}\langle\mathbf{k}\rangle_{\varsigma}^{h}}{\left\langle\mu_{\gamma}\right\rangle_{\varsigma}^{h}}\left[\frac{P_{\gamma^{+}}-P_{\gamma^{-}}}{\delta x_{\varsigma}}+\left\langle\rho_{\gamma} g\right\rangle_{\varsigma}^{a} z_{g \varsigma}\right] \text { for } \gamma=l, g ; j=w, a ; \text { and } \varsigma=E, W, S, N, B, T \\
J_{\gamma \varsigma^{\prime}}^{j}=-\left\langle\tau_{\gamma} \phi_{D} \rho_{\gamma} s_{\gamma} \frac{M^{j}}{M_{\gamma}} D_{\gamma}^{j}\right\rangle_{\varsigma}^{h} \frac{\chi_{\gamma^{+}}^{j}-\chi_{\gamma \varsigma^{-}}^{j}}{\delta x_{\varsigma}} \text { for } \gamma=l, g ; j=w, a ; \text { and } \varsigma=E, W, S, N, B, T
\end{gathered}
$$

and where \langle\rangle$_{\varsigma}^{h}$ represents harmonic interfacial averaging at surface $\varsigma,\langle\rangle_{\varsigma}^{u w}$ represents upwind or donor cell interfacial averaging at surface $\varsigma,\langle\rangle_{\varsigma}^{a}$ represents harmonic interfacial averaging at surface $\varsigma$, and $\sum_{\varsigma=\mathrm{W}, \mathrm{E}, \mathrm{S}, \mathrm{N}, \mathrm{T}, \mathrm{B}}$ is the summation over all node surfaces (west, east, south, north, top, bottom).

The mass conservation equations are discretized in time using a fully implicit scheme according to Equation (3.7), where the time levels are indicated with superscripts. The primary unknowns for the mass conservation equations are intrinsic properties at node volume centroids (node grid point) for time level $t+\delta t$. 


$$
\begin{aligned}
& \bar{V}\left[\frac{\left\{\bar{M}^{j}\right\}^{t+\delta t}-\left\{\bar{M}^{j}\right\}^{t}}{\delta t}\right]=\bar{V}\left\{\dot{m}^{j}\right\}^{t+\frac{\delta t}{2}} \\
& -\sum_{\varsigma=\mathrm{W}, \mathrm{E}, \mathrm{S}, \mathrm{N}, \mathrm{T}, \mathrm{B}}\left[\sum_{\gamma=1, \mathrm{~g}}\left(\left\{\mathrm{~F}_{\gamma \varsigma}^{\mathrm{j}}\right\}^{t+\delta t}+\left\{J_{\gamma \varsigma}^{\mathrm{j}}\right\}^{t+\delta t}\right)\right] \mathrm{A}_{\varsigma} \text { for } j=w, a
\end{aligned}
$$

Converting Equation (3.7) to residual form, where $\bar{R}^{j}$ is the residual for the mass conservation equation of component $j$ yields the expression shown in Equation (3.8):

$$
\begin{aligned}
& \left\{\bar{R}^{j}\right\}^{t+\delta t}=\bar{V}\left[\frac{\left\{\bar{M}^{j}\right\}^{t+\delta t}-\left\{\bar{M}^{j}\right\}^{t}}{\delta t}\right]-\bar{V}\left\{\dot{m}^{j}\right\}^{t+\frac{\delta t}{2}} \\
& +\sum_{\varsigma=\mathrm{W}, \mathrm{E}, \mathrm{S}, \mathrm{N}, \mathrm{T}, \mathrm{B}}\left[\sum_{\gamma=1, \mathrm{~g}}\left(\left\{\mathrm{~F}_{\gamma_{\varsigma}}^{\mathrm{j}}\right\}^{t+\delta t}+\left\{J_{\gamma_{\varsigma}}^{\mathrm{j}}\right\}^{t+\delta t}\right)\right] \mathrm{A}_{\varsigma} \text { for } j=w, a
\end{aligned}
$$

\subsection{Energy Conservation Equation}

The energy conservation equation shown in Equation (2.7) is discretized by assuming a piecewise profile to express the variation in primary variables between node points and integrating over the node volume. The thermal capacitance terms (that is, terms on the left side) and energy source terms are integrated over the node volume according to Equations (3.9) and (3.10), respectively:

$$
\frac{\partial}{\partial t}\left[\int_{\bar{V}} \bar{E} d \bar{V}\right]=\frac{\partial}{\partial t}[\bar{E} \bar{V}]
$$

where

$$
\begin{gathered}
\bar{E}=\sum_{\gamma=\ell, g}\left(\phi_{D} \rho_{\gamma} s_{\gamma} u_{\gamma}\right)+\left(1-\phi_{T}\right) \rho_{s} u_{s}+\left(\phi_{T}-\phi_{D}\right) \rho_{\ell} u_{\ell} \\
\int_{\bar{V}}\left[\sum_{j=w, a}\left(h^{j} \dot{m}^{j}\right)+\dot{Q}\right] d \bar{V}=\left[\sum_{j=w, a}\left(h^{j} \dot{m}^{j}\right)+\dot{Q}\right] \bar{V}
\end{gathered}
$$

Thermal energy is transferred through advection and diffusion. Spatial discretization of the advective and diffusive thermal flux proceeds by first converting the volumetric integral of flux over a control volume to a surface integral using Green's theorem (Kreyszig 1979) according to Equation (3.11). Surface integrals are approximated by discretizing the control volume surfaces into node surfaces and summing the contributions to heat flux over the node surfaces, according to Equation (3.12). 


$$
\begin{aligned}
& \int_{\bar{V}}\left[-\sum_{\gamma=l, g}\left(\nabla \rho_{\gamma} h_{\gamma} \mathbf{q}_{\gamma}+\sum_{j=w, a}\left(\nabla h^{j} \mathbf{J}_{\gamma}^{j}\right)+\nabla\left(\mathbf{k}_{e} \nabla T\right)\right)\right] d \bar{V} \\
& =\int_{\Gamma}\left[-\sum_{\gamma=l, g}\left(\rho_{\gamma} h_{\gamma} \mathbf{q}_{\gamma}+\sum_{j=w, a}\left(h^{j} \mathbf{J}_{\gamma}^{j}\right)+\left(\mathbf{k}_{e} \nabla T\right)\right)\right] \mathbf{n} d \Gamma
\end{aligned}
$$

where

$$
\begin{gathered}
\mathbf{q}_{\gamma}=-\frac{k_{r \gamma} \mathbf{k}}{\mu_{\gamma}}\left(\nabla P_{\gamma}+\rho_{\gamma} g \mathbf{z}_{g}\right) \text { for } \gamma=l, g \\
\mathbf{J}_{\gamma}^{j}=-\tau_{\gamma} \phi_{D} \rho_{\gamma} s_{\gamma} \frac{M^{j}}{M_{\gamma}} D_{\gamma}^{j} \nabla \chi_{\gamma}^{j} \text { for } \gamma=l, g \text { and } j=w, a \\
\int_{\Gamma}\left[-\sum_{\gamma=l, g}\left(\rho_{\gamma} h_{\gamma} \mathbf{q}_{\gamma}+\sum_{j=w, a}\left(h^{j} \mathbf{J}_{\gamma}^{j}\right)+\left(\mathbf{k}_{e} \nabla T\right)\right)\right] \mathbf{n} d \Gamma \\
=\sum_{\varsigma=W, E, S, N, B, T}\left[-\sum_{\gamma=l, g}\left(\left(\rho_{\gamma} h_{\gamma} \mathbf{q}_{\gamma}\right)_{\varsigma}+\sum_{j=w, a}\left(h^{j} \mathbf{J}_{\gamma}^{j}\right)_{\varsigma}+\left(\mathbf{k}_{e} \nabla T\right)_{\varsigma}\right)\right] A_{\varsigma}
\end{gathered}
$$

where

$$
\begin{gathered}
q_{\gamma \varsigma}^{j}=-\frac{\left\langle k_{r \gamma}\right\rangle_{\varsigma}^{u w}\langle\mathbf{k}\rangle_{\varsigma}^{h}}{\left\langle\mu_{\gamma}\right\rangle_{\varsigma}^{h}}\left[\frac{P_{\gamma^{+}}-P_{\gamma^{-}}}{\delta x_{\varsigma}}+\left\langle\rho_{\gamma} g\right\rangle_{\varsigma}^{a} z_{g \varsigma}\right] \text { for } \gamma=l, g ; j=w, a ; \text { and } \varsigma=E, W, S, N, B, T \\
J_{\gamma \zeta^{j}}^{j}=-\left\langle\tau_{\gamma} \phi_{D} \rho_{\gamma s_{\gamma}} \frac{M^{j}}{M_{\gamma}} D_{\gamma}^{j}\right\rangle_{\varsigma}^{h} \frac{\chi_{\gamma^{+}}^{j}-\chi_{\gamma^{-}}^{j}}{\delta x_{\varsigma}} \text { for } \gamma=l, g ; j=w, a ; \text { and } \varsigma=E, W, S, N, B, T
\end{gathered}
$$

The diffusive term of Equation (3.12) is computed using a user-defined interfacial average for the effective thermal conductivity, where the default form is harmonic averaging, according to Equation (3.13). The advective components of Equation (3.12) are computed using upwind (donor cell) averaging, according to Equations (3.14) and (3.15).

$$
\left(\mathbf{k}_{e} \nabla T\right)_{\varsigma}=\left\langle\mathbf{k}_{e}\right\rangle_{\varsigma}^{h} \frac{T_{\varsigma^{+}}-T_{\varsigma^{-}}}{\delta x_{\varsigma}} \text { for } \varsigma=E, W, S, N, B, T
$$




$$
\left(\rho_{\gamma} h_{\gamma} \mathbf{q}_{\gamma}\right)_{\varsigma}=\left(\rho_{\gamma} h_{\gamma}\right)_{\varsigma^{-}} \max \left[q_{\gamma_{\varsigma^{-}}}, 0\right]-\left(\rho_{\gamma} h_{\gamma}\right)_{\varsigma^{+}} \max \left[-q_{\gamma \varsigma^{+}}, 0\right]
$$

for $\gamma=l, g$ and $\varsigma=E, W, S, N, B, T$

$$
\left(h^{j} \mathbf{J}_{\gamma}^{j}\right)_{\varsigma}=\left(h^{j}\right)_{\varsigma^{-}} \max \left[J_{\gamma-}^{j}, 0\right]-\left(h^{j}\right)_{\varsigma^{+}} \max \left[-J_{\gamma^{+}}^{j}, 0\right]
$$

for $\gamma=l, g$ and $\varsigma=E, W, S, N, B, T$

As with the mass conservation equations, the energy conservation equations are discretized in time using a fully implicit scheme, according to Equation (3.16), where the time levels are indicated with superscripts. The primary unknowns for the energy equation are the temperatures at the node centroids (node grid points) for time level $\mathrm{t}+\delta \mathrm{t}$. In residual form, Equation (3.16) appears as shown in Equation (3.17).

$$
\begin{gathered}
\bar{V}\left[\frac{\{\bar{E}\}^{t+\delta t}-\{\bar{E}\}^{t}}{\delta t}\right]=\bar{V}\left\{\sum_{j=w, a}\left(h^{j} \dot{m}^{j}\right)+\dot{Q}\right\}^{t+\frac{\delta t}{2}}=\sum_{\varsigma=W, E, S, N, B, T}\left[\left\{\left(\mathbf{k}_{e} \nabla T\right)_{\varsigma}\right\}^{t+\delta t}\right] A_{\varsigma} \\
-\sum_{\varsigma=W, E, S, N, B, T}\left[\sum_{\gamma=l, g}\left[\left\{\left(\rho_{\gamma} h_{\gamma} \mathbf{q}_{\gamma}\right)_{\varsigma}\right\}^{t+\delta t}-\sum_{j=w, a}\left\{\left(h^{j} \mathbf{J}_{\gamma}^{j}\right)_{\varsigma}\right\}^{t+\delta t}\right)\right] A_{\varsigma} \\
\left\{\bar{R}_{e}\right\}^{t+\delta t}=\bar{V}\left[\frac{\{\bar{E}\}^{t+\delta t}-\{\bar{E}\}^{t}}{\delta t}\right]-\bar{V}\left\{\sum_{j=w, a}\left(h^{j} \dot{m}^{j}\right)+\dot{Q}\right\}^{t+\frac{\delta t}{2}} \\
-\sum_{\varsigma=W, E, S, N, B, T}\left[\left\{\left(\mathbf{k}_{e} \nabla T\right)_{\varsigma}\right\}^{t+\delta t}\right] A_{\varsigma} \\
+\sum_{\varsigma=W, E, S, N, B, T}\left[\sum_{\gamma=l, g}\left(\left\{\left(\rho_{\gamma} h_{\gamma} \mathbf{q}_{\gamma}\right)_{\varsigma}\right\}^{t+\delta t}-\sum_{j=w, a}\left\{\left(h^{j} \mathbf{J}_{\gamma}^{j}\right)_{\varsigma}\right\}^{t+\delta t}\right] A_{\varsigma}\right.
\end{gathered}
$$

\subsection{Solute Conservation Equation}

The solute mass conservation equation, shown in Equation (2.10) is discretized by assuming a piecewise profile for the solute concentration between node points and integrating over the node volume. The advection and diffusion-dispersion transport terms of the solute mass conservation equation are combined following the power-law scheme of Patankar (1980). Integration of the accumulation, source, and decay terms for solute mass over the node volume proceeds according to Equation (3.18).

$$
\int_{\bar{V}}\left(\frac{\partial C}{\partial t}-\dot{m}^{C}-\dot{R}^{C} C\right) d \bar{V}=\frac{\partial C}{\partial t} \bar{V}-\dot{m}^{C} \bar{V}-\dot{R}^{C} C \bar{V}
$$


Solute transport fluxes are computed between node points and comprise advective and diffusivedispersive components. Integration of the flux terms proceeds by converting volumetric integrals to surface integrals following Green's theorem (Kreyszig 1979), according to Equation (3.19). Surface integrals are approximated by discretizing the control volume surfaces into node surfaces and summing the contributions to solute transport over the node surfaces according to Equation (3.20). The diffusivedispersive term of Equation (3.20) is computed using a user-defined interfacial average where the default scheme is harmonic averaging, according to Equation (3.21).

$$
\begin{aligned}
& \int_{\bar{V}}\left[-\sum_{\gamma=l, g}\left(\nabla\left[C_{\gamma} q_{\gamma}\right]\right)+\sum_{\gamma=l, g}\left(\nabla\left[\left(\tau_{\gamma} s_{\gamma} \phi_{D} D_{\gamma}^{C}+s_{\gamma} \phi_{D} \mathbf{D}_{h \gamma}\right) \nabla C_{\gamma}\right]\right)\right] d \bar{V} \\
& =\int_{\Gamma}\left[-\sum_{\gamma=l, g}\left(C_{\gamma} q_{\gamma}\right)+\sum_{\gamma=l, g}\left(\left(\tau_{\gamma} s_{\gamma} \phi_{D} D_{\gamma}^{C}+s_{\gamma} \phi_{D} \mathbf{D}_{h \gamma}\right) \nabla C_{\gamma}\right)\right] \cdot \mathbf{n} d \Gamma
\end{aligned}
$$

where

$$
\begin{gathered}
\mathbf{q}_{\gamma}=-\frac{k_{r \gamma} \mathbf{k}}{\mu_{\gamma}}\left(\nabla P_{\gamma}+\rho_{\gamma} g \mathbf{z}_{g}\right) \text { for } \gamma=\ell, g \\
\int_{\Gamma}\left[-\sum_{\gamma=l, g}\left(C_{\gamma} q_{\gamma}\right)+\sum_{\gamma=l, g}\left(\left(\tau_{\gamma} s_{\gamma} \phi_{D} D_{\gamma}^{C}+s_{\gamma} \phi_{D} \mathbf{D}_{h \gamma}\right) \nabla C_{\gamma}\right)\right] \cdot \mathbf{n} d \Gamma \\
=\sum_{\varsigma=W, E, S, N, B, T}\left[-\sum_{\gamma=l, g}\left(C_{\gamma} q_{\gamma}\right)_{\varsigma}+\sum_{\gamma=l, g}\left(\left(\tau_{\gamma} s_{\gamma} \phi_{D} D_{\gamma}^{C}+s_{\gamma} \phi_{D} \mathbf{D}_{h \gamma}\right) \nabla C_{\gamma}\right)_{\varsigma}\right] A_{\varsigma}
\end{gathered}
$$

where

$$
\begin{gathered}
q_{\gamma \varsigma}^{j}=-\frac{\left\langle k_{r \gamma}\right\rangle_{\varsigma}^{u w}\langle\mathbf{k}\rangle_{\varsigma}^{h}}{\left\langle\mu_{\gamma}\right\rangle_{\varsigma}^{h}}\left[\frac{P_{\gamma \varsigma^{+}}-P_{\gamma \varsigma^{-}}}{\delta x_{\varsigma}}+\left\langle\rho_{\gamma} g\right\rangle_{\varsigma}^{a} z_{g \varsigma}\right] \text { for } \gamma=l, g ; j=w, a ; \text { and } \varsigma=E, W, S, N, B, T \\
\left(\left(\tau_{\gamma} s_{\gamma} \phi_{D} D_{\gamma}^{C}+s_{\gamma} \phi_{D} \mathbf{D}_{h \gamma}\right) \nabla C_{\gamma}\right)_{\varsigma}=\left\langle\tau_{\gamma} s_{\gamma} \phi_{D} D_{\gamma}^{C}+s_{\gamma} \phi_{D} \mathbf{D}_{h \gamma}\right\rangle_{\varsigma}^{h} \frac{C_{\gamma \varsigma^{+}}-C_{\gamma \varsigma^{-}}}{\delta x_{\varsigma}} \\
\text { for } \gamma=l, g \text { and } \varsigma=E, W, S, N, B, T
\end{gathered}
$$

Solution of the solute transport equation depends on the local Peclet number that represents the ratio of advective transport to diffusive-dispersive transport, according to Equation (3.22)

$$
P e_{\gamma_{\varsigma}}=\frac{q_{\gamma \varsigma} \delta x_{\varsigma}}{\left\langle\tau_{\gamma} s_{\gamma} \phi_{D} D_{\gamma}^{C}+s_{\gamma} \phi_{D} \mathbf{D}_{h \gamma}\right\rangle_{\varsigma}^{h}}
$$


The power law scheme is based on the solute concentration profile for steady conditions with no sources or decay. For a Peclet number of zero, diffusion-dispersion transport dominates, and a linear profile of solute concentration occurs between two spatial points. For a Peclet number of one, advection and diffusion-dispersion equally contribute to solute transport. The solute concentration profile will be skewed toward an upstream solute concentration. For large Peclet numbers, advection transport dominates, and the upstream solute concentration defines the solute concentration profile between two spatial points. The power-law scheme closely approximates the exact solution for steady conditions without excessive computational expense. Solute flux from combined advective and diffusive-dispersive transport can be expressed using the power-law scheme according to Equation (3.23).

$$
\begin{aligned}
& \left.G_{\gamma_{\varsigma}}^{C}=-C_{\gamma_{\varsigma^{+}}}\left[\max \left(-q_{\gamma_{\varsigma}}, 0\right)+\left(D_{\gamma_{e}}^{C}\right)_{\varsigma} \max \left(1-\left(\frac{0.1\left|q_{\gamma_{\varsigma}}\right|}{\left(D_{\gamma_{e}}^{C}\right)_{\varsigma}}\right)\right)^{5}, 0\right)\right] \\
& \left.+C_{\gamma_{\varsigma^{-}}}\left[\max \left(q_{\gamma_{\varsigma}}, 0\right)+\left(D_{\gamma_{e}}^{C}\right)_{\varsigma} \max \left(1-\left(\frac{0.1\left|q_{\gamma_{\varsigma}}\right|}{\left(D_{\gamma_{e}}^{C}\right)_{\varsigma}}\right)\right)^{5}, 0\right)\right]
\end{aligned}
$$

for $\gamma=l, g$ and $\varsigma=W, E, S, N, B, T$

where

$$
\left(D_{\gamma_{e}}^{C}\right)_{\varsigma}=\frac{\left\langle\tau_{\gamma} s_{\gamma} \phi_{D} D_{\gamma}^{C}+s_{\gamma} \phi_{D} \mathbf{D}_{h \gamma}\right\rangle_{\varsigma}^{h}}{\delta x_{\varsigma}}
$$

The discretized solute concentration equation can be written in two compact forms. The first form uses the expressions of solute flux according to Equation (3.24). The second form uses a linear system format with coefficients for the solute concentrations according to Equation (3.25).

$$
\begin{gathered}
\sum_{\gamma=l, g}\left(G_{\gamma_{W}}^{C}-G_{\gamma_{E}}^{C}+G_{\gamma_{S}}^{C}-G_{\gamma_{N}}^{C}+G_{\gamma_{B}}^{C}-G_{\gamma_{T}}^{C}\right)=\frac{\partial C}{\partial t} \bar{V}-\dot{m}^{C} \bar{V}-\dot{R}^{C} C \bar{V} \\
a_{P} C_{P}-\sum_{\varsigma=W, E, S, N, B, T} a_{\varsigma} C_{\varsigma}=\frac{\partial C}{\partial t} \bar{V}-\dot{m}^{C} \bar{V}-\dot{R}^{C} C \bar{V}
\end{gathered}
$$

where

$$
a_{\varsigma}=\sum_{\gamma=l, g} a_{\gamma_{\varsigma}}
$$




$$
\begin{gathered}
a_{\gamma_{P}}=\sum_{\varsigma=W, E, S, N, B, T} a_{\gamma_{\varsigma}}+\sum_{\varsigma=E, N, T} q_{\gamma_{\varsigma}}-\sum_{\varsigma=W, S, B} q_{\gamma_{\varsigma}} \\
\left.a_{\gamma_{\varsigma}}=\max \left(q_{\gamma_{\varsigma}}, 0\right)+\left(D_{\gamma_{e}}^{C}\right)_{\varsigma} \max \left(1-\left(\frac{0.1\left|q_{\gamma_{\varsigma}}\right|}{\left(D_{\gamma_{e}}^{C}\right)_{\varsigma}}\right)\right)^{5}, 0\right) \text { for } \gamma=l, g \text { and } \varsigma=W, S, B \\
\left.a_{\gamma_{\varsigma}}=\max \left(-q_{\gamma_{\varsigma}}, 0\right)+\left(D_{\gamma_{e}}^{C}\right)_{\varsigma} \max \left(1-\left(\frac{0.1\left|q_{\gamma_{\varsigma}}\right|}{\left(D_{\gamma_{e}}^{C}\right)_{\varsigma}}\right)\right)^{5}, 0\right) \text { for } \gamma=l, g \text { and } \varsigma=E, N, T .
\end{gathered}
$$

The solute mass conservation equation is discretized in time using a fully implicit scheme, according to Equation (3.26), in linear system format, where time levels are indicated with superscripts.

$$
\sum_{\varsigma=P, w, E, S, N, B, T} a_{\varsigma}\left\{C_{\varsigma}\right\}^{t+\delta t}=\frac{\left\{C_{p}\right\}^{t}}{\partial t} \bar{V}-\dot{m}^{C} \bar{V}
$$

where

$$
\begin{gathered}
a_{\varsigma}=\sum_{\gamma=l, g} a_{\gamma_{\varsigma}} \\
a_{\gamma_{P}}=\sum_{\varsigma=W, E, S, N, B, T} a_{\gamma_{\varsigma}}+\sum_{\varsigma=E, N, T} V_{\gamma_{\varsigma}}-\sum_{\varsigma=W, S, B} V_{\gamma_{\varsigma}}+\frac{\bar{V}}{\partial t}+\dot{R}^{C} \bar{V} \\
\left.a_{\gamma_{\varsigma}}=\max \left(q_{\gamma_{\varsigma}}, 0\right)+\left(D_{\gamma_{e}}^{C}\right)_{\varsigma} \max \left(1-\left(\frac{0.1\left|q_{\gamma_{\varsigma}}\right|}{\left(D_{\gamma_{e}}^{C}\right)_{\varsigma}}\right)\right)^{5}, 0\right) \text { for } \gamma=l, g \text { and } \varsigma=W, S, B \\
\left.a_{\gamma_{\varsigma}}=\max \left(-q_{\gamma_{\varsigma}}, 0\right)+\left(D_{\gamma_{e}}^{C}\right)_{\varsigma} \max \left(1-\left(\frac{0.1\left|q_{\gamma_{\varsigma}}\right|}{\left(D_{\gamma_{e}}^{C}\right)_{\varsigma}}\right)\right)^{5}, 0\right) \text { for } \gamma=l, g \text { and } \varsigma=E, N, T .
\end{gathered}
$$




\subsubsection{Newton-Raphson Linearization}

The discretized governing equations for component mass conservation and energy conservation, Equations (3.8) and (3.17), form a nonlinear set of algebraic equations. Nonlinear equation sets arise from the dependence of secondary variables on the primary unknowns. Cross dependencies of secondary variables in one governing equation with the primary variable unknown in other governing equations requires that this system of nonlinear equations be solved simultaneously. A primary assumption associated with the solute mass conservation equation is that solute concentrations are infinitely dilute. This assumption implies that fluid properties are independent of solute concentrations, which allows the solute conservation equations to be solved separately from the coupled flow and energy transport solutions. The discretized governing equations for solute mass conservation, Equation (3.26), form a linear set of algebraic equations that are solved directly following the solution of the coupled flow and energy transport system. The nonlinear situations in the coupled flow and energy transport system of equations are resolved through the application of the iterative Newton-Raphson technique.

The Newton-Raphson linearization technique is an iterative method for solving nonlinear algebraic equations of the form shown in Equation (3.27), where $f(x)$ is differentiable in $x$. The linearization concept approximates $f(x)$ with suitable tangents. Each iteration yields a new estimate of $x$ as the intersection of the tangent to the function $f(x)$ at the previous estimate of $x$ and the abscissa axis, according to Equation (3.28) in mathematical form. In this formulation, $f(x)$ is considered the equation residual. For convergent systems, the residual decreases quadratically with iteration. In multiple variable systems, as with the coupled flow and energy transport system, the scalar function, $f(x)$, is replaced with a vector function $\mathbf{R}(\mathbf{x})$, according to Equation (3.29). The vector function, $\mathbf{R}(\mathbf{x})$, represents the system of nonlinear algebraic equations produced from discretizing the conservation equations for component mass and energy, Equations (3.8) and (3.17). The vector of unknowns, $x$, represents the set of primary variables for the system that are determined by the operational mode and phase conditions. Equation (3.29) can be rewritten in terms of increments to the primary variables, according to Equation (3.30). The partial derivatives shown in Equation (3.30) form the Jacobian matrix.

$$
\begin{gathered}
f(x)=0 \\
x^{n+1}=x^{n}-\frac{f(x)}{f^{\prime}(x)} \\
x^{n+1}=x^{n}-\frac{\mathbf{R}(\mathbf{x})}{\mathbf{R}^{\prime}(\mathbf{x})} \\
\frac{\partial \mathbf{R}(\mathbf{x})}{\partial \mathbf{x}} \Delta \mathbf{x}=-\mathbf{R}(\mathbf{x})
\end{gathered}
$$

To simplify these discussions, a one-dimensional system involving the solution of the water mass, air mass, and energy conservation equations is considered. The system of linear equations that result from applying the Newton-Raphson linearization technique to this system of nonlinear algebraic equations is 
shown in Equation (3.31) for a computational domain with $n$ nodes. In Equation (3.31), each Jacobian matrix element represents a block matrix of order three, according to Equation (3.32). Each unknown vector element represents a vector of increments to the primary variables of order three, according to Equation (3.33). Each solution vector element represents a vector of equation residuals of order three, according to Equation (3.34).

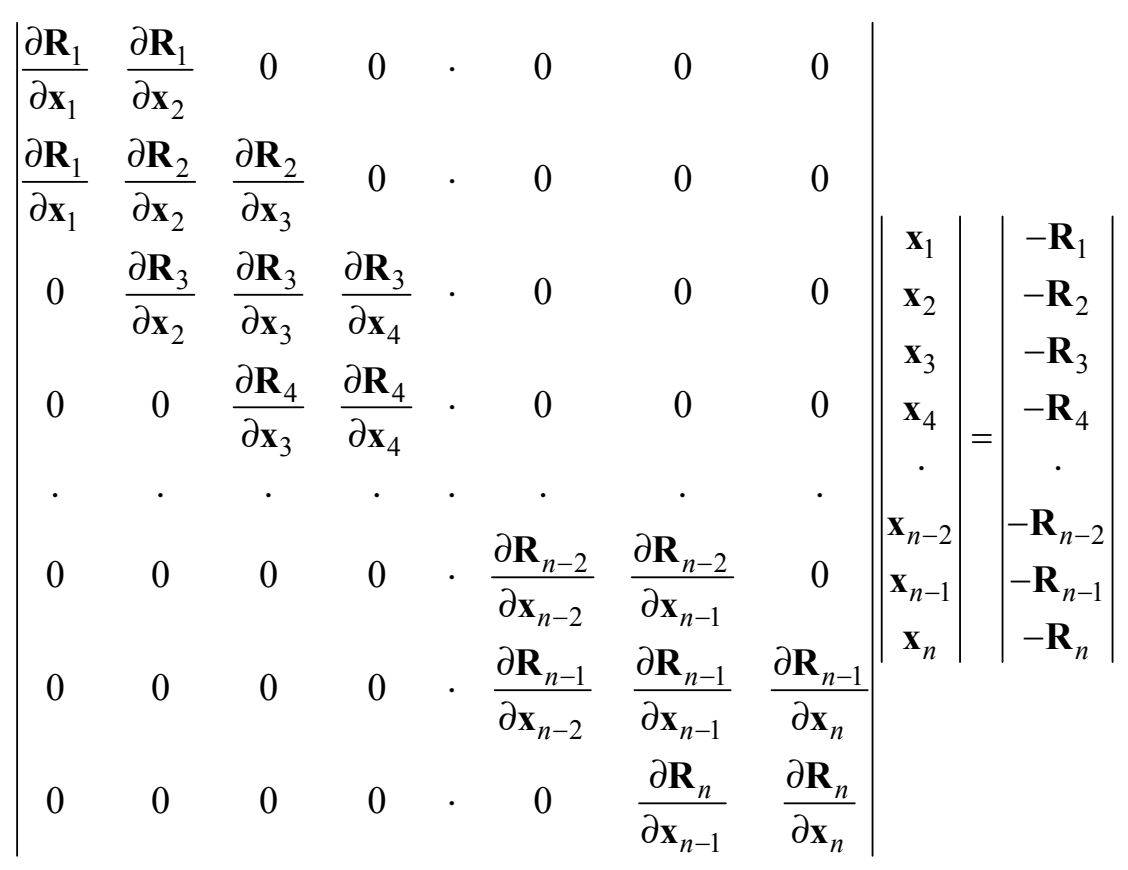

$$
\begin{aligned}
& \frac{\partial \mathbf{R}_{k}}{\partial \mathbf{x}_{l}}=\left|\begin{array}{ccc}
\frac{\partial R_{k}^{w}}{\partial P V_{l}^{w}} & \frac{\partial R_{k}^{w}}{\partial P V_{l}^{a}} & \frac{\partial R_{k}^{w}}{\partial P V_{l}^{e}} \\
\frac{\partial R_{k}^{a}}{\partial P V_{l}^{w}} & \frac{\partial R_{k}^{a}}{\partial P V_{l}^{a}} & \frac{\partial R_{k}^{a}}{\partial P V_{l}^{e}} \\
\frac{\partial R_{k}^{e}}{\partial P V_{l}^{w}} & \frac{\partial R_{k}^{e}}{\partial P V_{l}^{a}} & \frac{\partial R_{k}^{e}}{\partial P V_{l}^{e}}
\end{array}\right| \\
& -\mathbf{x}_{l}=\left|\begin{array}{l}
\Delta P V_{l}^{w} \\
\Delta P V_{l}^{a} \\
\Delta P V_{l}^{e}
\end{array}\right| \\
& -\mathbf{R}_{k}=\left|\begin{array}{c}
-R_{k}^{w} \\
-R_{k}^{a} \\
-R_{k}^{e}
\end{array}\right|
\end{aligned}
$$


For a 2-dimensional (2-D) system involving the solution of the water mass, air mass, and energy conservation equations, the Jacobian matrix contains two extra bands of block matrixes: one below and one above the diagonal band. These extra bands are located one-half-band width from the main diagonal band, where the half band width equaled the lesser of the number of nodes per row or column for a 2-D grid. A 3-dimensional (3-D) grid contains four extra bands of block matrixes, two below and two above the diagonal band. The farthest bands are one-half-band width from the main diagonal band, where the half band width equaled the least number of nodes in a plane. For example, a 3-D Cartesian grid with 20 nodes in the $\mathrm{X}$ coordinate direction, 30 nodes in the $\mathrm{Y}$ coordinate direction, and 40 nodes in the $\mathrm{Z}$ coordinate direction has a half-band width of 600 .

The Newton-Raphson scheme has two major computational components. The first involves computing the Jacobian matrix and solution vector elements; the second involves solving the resulting linear system of equations. During each iteration, both components are required. The Jacobian matrix elements are essentially partial derivatives of the equation residuals with respect to the primary variables. These partial derivatives are computed numerically as exemplified in Equation (3.35)

$$
\frac{\partial R_{k}^{j}}{\partial P V_{l}^{i}} \cong \frac{R_{k}^{j}\left|P V_{l}^{i}+\Delta P V_{l}^{i}-R_{k}^{j}\right| P V_{l}^{i}}{\Delta P V_{l}^{i}} .
$$

For robustness and directness in coding, numerical evaluation of these partial derivatives was chosen over analytical evaluation. The numerical evaluation scheme requires that the equation residuals be evaluated with the most current values of the primary variables and with each primary variable slightly incremented. Increments to the primary variables are critical parameters for implementing the NewtonRaphson linearization scheme using numerical derivatives. Excessively large increments can lead to nonconvergent solutions because the tangents to the residual equations are computed incorrectly. In contrast, excessively small increments can result in changes in the equation residuals that are below the precision limit.

Each Newton-Raphson iteration procedure begins with the start of a new time step or after a convergence failure and reduction in time step. Each iteration begins by executing logic algorithms that determine the primary variable set from phase conditions and fix the primary variable increments. The next step is to calculate all secondary variables with the current primary variables and with each primary variable incremented. Following this, all flux variables are evaluated with the current primary variable and associated secondary variables and then repeated with the incremented primary and associated secondary variables. With the secondary variables and fluxes evaluated, the residuals to the governing equations are computed using current and incremented values of the primary, secondary, and flux variables. The current and incremented equations residuals are then used to compute the partial derivatives that comprise the Jacobian matrix and the solution vector.

The resulting system of linear equations is then solved with a direct or an iterative linear system solver with the unknowns as increments to the primary variables. Converged solutions are recognized by comparing the maximum normalized increment to the primary variables against a convergence criterion (a user-specified limit). A Newton-Raphson iteration ends by updating the primary variables with the latest computed primary increments. If the largest normalized primary variable increment exceeds the convergence limit, another iteration is executed. If the solution is convergent, the solute transport 
equations are solved or a new time step initiated. In a non-convergent solution the time step is reduced, the primary variables are reset to the most recent converged values, and the time step is reinitiated.

\subsubsection{Linear System Solvers}

The system of linear equations that results from the discretization of the governing partial differential equations over a particular computational domain always has a banded structure, where the number of off diagonal bands equals the dimensions of the computational domain. Elements within the Jacobian coefficient are actually submatrixes, where the submatrix order equals the number of solved coupled governing equations. For example, the solution of only the water conservation equation produces single element submatrixes, whereas the simultaneous solution of the three mass conservation equations with the energy equation yields four-by-four submatrixes. These structured Jacobian coefficient matrixes arise from the element sequencing scheme incorporated into the STORM simulator and the limitations on the computational domain structures. The matrix sequencing or numbering algorithms are designed to minimize the largest half-band width for a given computational domain and solution option combination. The sequencing algorithms are designed for orthogonal grid systems that are six-surfaced in three dimensions or four-sided in two dimensions.

Two linear equation solvers from the public domain are available with the STORM simulator: 1) a direct-banded matrix algorithm and 2) SPLIB, a library of sparse iterative solvers that are primarily conjugate gradient algorithms. Because the banded matrix algorithm requires more memory than the conjugate gradient iterative algorithms, it is generally more appropriate for small to moderately sized Jacobian matrixes (order less than 35,000). For larger problems the SPLIB solver library should be used.

\subsubsection{Direct solver}

The banded matrix solution algorithm was extracted from the LINPACK subroutines (Dongarra et al. 1979) for general nonsymmetric band matrixes. The algorithm operates on band matrixes by decomposing the matrix into upper and lower triangular matrixes. The product of the lower triangular matrix times the upper triangular matrix equals the original band matrix (that is, $\mathbf{A}=\mathbf{L} \cdot \mathbf{U}$ where $\mathbf{A}$ is the band matrix, $\mathbf{L}$ is the lower triangular matrix, and $\mathbf{U}$ is the upper triangular matrix). The system of linear equations, $\mathbf{A} \cdot \mathbf{x}=\mathbf{b}$, is solved with the preceding decomposition or factorization by solving successively $\mathbf{L}(\mathbf{U} \cdot \mathbf{x})=\mathbf{b}$. This factorization procedure produces nonzero elements outside the bands of the original band matrix. If $m_{1}$ equals the half bandwidth of the Jacobian coefficient matrix (the STORM simulator produces band matrixes with equal lower and upper bandwidths), the two triangular factors have bandwidths of $m_{1}$ and $2 m_{1}$. Storage must be provided for the extra $m_{1}$ diagonals. This requirement is illustrated for a 1-dimensional problem of five nodes and two solved mass conservation equations. The Jacobian coefficient matrix for this problem appears as shown in Equation (3.36). The band storage requires $3 \mathrm{~m}_{1}+1=10$ rows of storage arranged as shown in Equation (3.37). The * indicates elements that are never referenced but for which storage space must be provided. The + indicates elements that may be occupied during the factorization process. The original Jacobian coefficient matrix is referred to as $\mathbf{A}$ and its storage counterpart as $\mathbf{a}$. The columns of $\mathbf{A}$ are stored in the columns of $\mathbf{a}$, and the diagonals of $\mathbf{A}$ are stored in the rows of $\mathbf{a}$, so the principal diagonal is stored in row $2 \mathrm{~m}_{1}+1$ of $\mathbf{a}$. 


\subsubsection{Iterative solvers}

SPLIB is a library of sparse iterative solvers with preconditioners for rapid prototyping of solvers for nonsymmetric linear systems of equations (Bramley and Wang 1995). SPLIB implements several incomplete factorization based preconditioning methods that must access the entries of the coefficient matrix A. That in turn means that some common data structure must be settled upon. The data structure used in SPLIB is CSR (compressed sparse row). This is the transpose of the data structure used for the Harwell/Boeing (HB) collection of test matrixes. CSR stores the nonzeros of the matrix, row by row, in a singly indexed array $\mathbf{A}$ of double precision numbers (real*8 in Fortran). Another array, COLIND, contains the column index of each corresponding entry in the A array. A third integer array, ROWPTR, contains pointers to the beginning of each row with an additional pointer to the first index following the nonzeros of the matrix $\mathbf{A}$. If $\mathrm{NNZ}$ is the number of nonzeros and $\mathrm{N}$ is the order of the matrix, $\mathbf{A}$ and COLIND have length NNZ and ROWPTR has length N. Although this storage format requires three coefficient matrixes, the storage requirements for large sparse systems (such as those for large multidimensional problems) are greatly reduced over those for the banded matrix solution scheme.

$$
\begin{aligned}
\mathbf{A} & =\left|\begin{array}{cccccccccc}
x_{1,1} & x_{1,2} & x_{1,3} & x_{1,4} & 0 & 0 & 0 & 0 & 0 & 0 \\
x_{2,1} & x_{2,2} & x_{2,3} & x_{2,4} & 0 & 0 & 0 & 0 & 0 & 0 \\
x_{3,1} & x_{3,2} & x_{3,3} & x_{3,4} & x_{3,5} & x_{3,6} & 0 & 0 & 0 & 0 \\
x_{4,1} & x_{4,2} & x_{4,3} & x_{4,4} & x_{4,5} & x_{4,6} & 0 & 0 & 0 & 0 \\
0 & 0 & x_{5,3} & x_{5,4} & x_{5,5} & x_{5,6} & x_{5,7} & x_{5,8} & 0 & 0 \\
0 & 0 & x_{6,3} & x_{6,4} & x_{6,5} & x_{6,6} & x_{6,7} & x_{6,8} & 0 & 0 \\
0 & 0 & 0 & 0 & x_{7,5} & x_{7,6} & x_{7,7} & x_{7,8} & x_{7,9} & x_{7,10} \\
0 & 0 & 0 & 0 & x_{8,5} & x_{8,6} & x_{8,7} & x_{8,8} & x_{8,9} & x_{8,10} \\
0 & 0 & 0 & 0 & 0 & 0 & x_{9,7} & x_{9,8} & x_{9,9} & x_{9,10} \\
0 & 0 & 0 & 0 & 0 & 0 & x_{10,7} & x_{10,8} & x_{10,9} & x_{10,10}
\end{array}\right| \\
\mathbf{a} & =\left|\begin{array}{ccccccccccc}
* & * & * & * & * & * & + & + & + & + \\
* & * & * & * & * & + & + & + & + & + \\
* & * & * & * & + & + & + & + & + & + \\
* & * & * & x_{1,4} & 0 & x_{3,6} & 0 & x_{5,8} & 0 & x_{7,10} \\
* & * & x_{1,3} & x_{2,4} & x_{3,5} & x_{4,6} & x_{5,7} & x_{6,8} & x_{7,9} & x_{8,10} \\
* & x_{1,2} & x_{2,3} & x_{3,4} & x_{4,5} & x_{5,6} & x_{6,7} & x_{7,8} & x_{8,9} & x_{9,10} \\
x_{1,1} & x_{2,2} & x_{3,3} & x_{4,4} & x_{5,5} & x_{6,6} & x_{7,7} & x_{8,8} & x_{9,9} & x_{10,10} \\
x_{2,1} & x_{3,2} & x_{4,3} & x_{5,4} & x_{6,5} & x_{7,6} & x_{8,7} & x_{9,8} & x_{10,9} & * \\
x_{3,1} & x_{4,2} & x_{5,3} & x_{6,4} & x_{7,5} & x_{8,6} & x_{9,7} & x_{10,8} & * & * \\
x_{4,1} & 0 & x_{6,3} & 0 & x_{8,5} & 0 & x_{10,7} & * & * & *
\end{array}\right|
\end{aligned}
$$

SPLIB implements 13 iterative solvers, primarily modern conjugate gradient methods, and seven preconditioning methods. By default, STORM uses the biconjugate gradients stabilized (CGSTAB) method (van der Vorst 1992). This method is a generalization of the method of conjugate gradients for 
asymmetric systems combined with minimal residual steps. The preconditioner used in SPLIB is incomplete LU [ILU(s)] factorization of s levels of fill (Meijerink and van der Vorst 1977). The s levels of fill in ILU(s) refers to the amount of fill-in allowed during the incomplete factorization. Using $\mathrm{s}=0$ gives upper and lower triangular factors $\mathrm{L}$ and $\mathrm{U}$ such that $\mathrm{L}+\mathrm{U}$ has the same sparsity pattern as $\mathbf{A}$. Using $\mathrm{s}=\mathrm{n}$ gives the full LU factorization of $\mathbf{A}$ without pivoting. Usually, increasing s provides a better quality preconditioner (in terms of reducing the number of iterations required) but at the cost of increasing storage. The recommended usage is to try a small value of $\mathrm{s}$, and increase it if the iterative method fails, or if it requires an excessive number of iterations. The default value used in STORM is $\mathrm{s}=1$.

\subsection{Geochemical Reactions}

The fully coupled reactive transport mass balance equation may be derived by combining the solute transport mass balance equation with the geochemical reaction mass balance equation. Combining Equations (2.17) and (2.20) yields the solute mass conservation equation including geochemical reactions:

$$
\begin{gathered}
\frac{\partial}{\partial t}\left[\phi_{D} \rho_{\ell}\left(s_{g} K_{H}^{\prime-1}+s_{\ell}\right) C_{i}^{\ell}\right]= \\
\nabla \bullet\left[-\phi_{D} \rho_{\ell}\left(s_{g} \mathbf{V}_{g} K_{H}^{\prime-1}+s_{\ell} \mathbf{V}_{\ell}\right) C_{i}^{\ell}+\phi_{D} \rho_{\ell}\left(s_{g} \mathbf{D}_{i}^{g} K_{H}^{\prime-1}+s_{\ell} \mathbf{D}_{i}^{\ell}\right) \cdot \nabla C_{i}^{\ell}\right] \\
+\sum_{j=1}^{N_{e}} v_{i j} W_{j}^{e}+\sum_{j=1}^{N_{k}} v_{i j} W_{j}^{k}
\end{gathered}
$$

In matrix form, Equation (3.38) can be written as

$$
\frac{\partial}{\partial t}\left[\phi_{D} \rho_{\ell}\left(s_{g} K_{H}^{\prime-1}+s_{\ell}\right) \overrightarrow{\mathbf{C}}\right]=\overrightarrow{\mathbf{L}}(\overrightarrow{\mathbf{C}})+\mathbf{N} \overrightarrow{\mathbf{W}}
$$

where

$$
\begin{gathered}
\overrightarrow{\mathbf{L}}(\overrightarrow{\mathbf{C}})=\left[L_{1}\left(C_{1}\right), \ldots, L_{N_{s}}\left(C_{N_{s}}\right)\right]^{T} \\
L_{i}\left(C_{i}\right)=\nabla \bullet\left[-\phi_{D} \rho_{\ell}\left(s_{g} \mathbf{V}_{g} K_{H}^{\prime-1}+s_{\ell} \mathbf{V}_{\ell}\right) C_{i}^{\ell}+\phi_{D} \rho_{\ell}\left(s_{g} \mathbf{D}_{i}^{g} K_{H}^{\prime-1}+s_{\ell} \mathbf{D}_{i}^{\ell}\right) \cdot \nabla C_{i}^{\ell}\right] \\
\overrightarrow{\mathbf{W}}=\left(W_{1}^{e}, \ldots W_{N_{e}}^{e}, W_{1}^{k}, \ldots W_{N_{k}}^{k}\right)^{T}
\end{gathered}
$$




$$
\mathbf{N}=\left[\begin{array}{cccc}
v_{11} & . . & . . & v_{1, N_{r}} \\
\cdot & . . & . . & . \\
\cdot & . . & . . & . \\
\cdot & . . & . . & . \\
v_{N_{s}, 1} & . . & . \cdot & v_{N_{s}, N_{r}}
\end{array}\right]
$$

where $N_{s}$ is the number of species, $N_{e}$ is the number of equilibrium reactions, and $N_{k}$ is the number of kinetic reactions.

\subsubsection{Linear Transformation of the Reaction-Transport Equation}

Ortoleva et al. (1987) pointed out that a subsurface system usually contains reactions of different time scales; therefore, "straightforward numerical solution of the solute equations is impractical." To remedy the stiffness of Equation (3.39), a modification is needed to the equation that separates reactions with different time scales. As discussed in Section 2.3.1, geochemical models usually assume some reactions with a short time scale are equilibrium reactions and others are kinetic reactions. In most cases, separating reactions with different time scales is separating equilibrium reactions from kinetic reactions.

One approach to performing such separations is based on a canonical representation of reactions (Kirkner and Reeves 1988; Lichtner 1985; Reed 1982; Sevougian et al. 1993; Steefel and Lasaga 1994; Yeh and Tripathi 1991). That approach arbitrarily assigns one subset of species as the primary (or base) species and the others as the secondary species. Reactions are written in canonical form, that is, each reaction involves only one secondary species but requires several primary species (Lichtner 1985). Consequently, the rate of production/destruction of primary species due to reversible reactions is expressed as the weighted summary of secondary species with the number of moles of primary species in the secondary species as the weight. Adding the mass conservation equation of secondary species with that of the primary species leads to a mass conservation equation that does not contain a source/sink term of reversible reactions; it not only separates equilibrium from kinetic reactions but also reduces the number of nonlinear partial differential equations (PDE) from $N_{s}$ to $N_{s}-N_{e}$.

From the perspective of linear algebra, the canonical representation approach is equivalent to a linear transformation on aqueous species. The original variables-species concentrations are transformed to total concentrations of chemical components, a linear combination of relevant species. This generalization has led toward a more general approach to separating equilibrium from kinetic reactions.

\subsubsection{Q-R Decomposition}

Unlike the canonical representation approach, the Q-R decomposition approach does not bear the concepts of primary and secondary species; thus, it does not impose the constraint of the canonical form on reactions. Eliminating this constraint frees the user from the trivial but time-consuming work of writing every reaction in canonical form. The user has more time to consider whether the chemical model is physically relevant.

Given a reaction matrix $\mathbf{N}$, with columns that are linearly independent (that is, the reactions are linearly independent) with the dimension of $N_{s} \times N_{r}$, the reactions are arranged so the first $N_{e}$ columns 
represent equilibrium reactions and the rest are kinetic reactions. The independent assumption guarantees $N_{r} \leq N_{s}$. Matrix algebra states that $\mathbf{N}$ can be decomposed to

$$
\mathbf{N}_{N_{s} \times N_{r}}=\mathbf{Q}_{N_{s} \times N_{r}} \cdot\left[\begin{array}{c}
\mathbf{R}_{N_{s} \times N_{r}} \\
0_{N_{r} \times\left(N_{s}-N_{r}\right)}
\end{array}\right]
$$

(Golub and Van Loan 1983), where $\mathbf{Q}$ is an orthogonal matrix:

$$
\mathbf{Q}^{T}=\mathbf{Q}^{-1}
$$

and $\mathbf{R}$ is an upper-triangular matrix:

$$
\mathbf{R}_{N_{R} \times N_{R}}=\left[\begin{array}{ccccc}
r_{11} & \cdot & \cdots & \cdot & \cdot \\
0 & r_{22} & \cdots & \cdot & \cdot \\
0 & \cdot & \cdots & \cdot & \cdot \\
\cdot & \cdot & \cdots & \cdot & \cdot \\
0 & 0 & \cdots & 0 & r_{N_{R}, N_{R}}
\end{array}\right]
$$

Multiplying Equation (3.39) by $\mathbf{Q}^{T}$ gives

$$
\frac{\partial}{\partial t}\left[\phi_{D} \rho_{\ell}\left(s_{g} K_{H}^{\prime-1}+s_{\ell}\right) \mathbf{Q}^{T} \overrightarrow{\mathbf{C}}\right]=\mathbf{Q}^{T} \overrightarrow{\mathbf{L}}(\overrightarrow{\mathbf{C}})+\mathbf{R} \overrightarrow{\mathbf{W}}
$$

Denote

$$
\mathbf{Q}^{T} \overrightarrow{\mathbf{C}}=\vec{\chi}=\left(\chi_{1}, \ldots, \chi_{N_{e}}, \chi_{N_{e}+1}, \ldots, \chi_{N_{e}+N_{k}}, \chi_{N_{e}+N_{k}+1}, \ldots, \chi_{N_{s}}\right)^{T}
$$

and recognize that each $\chi_{i}$ is a linear combination of $\left(C_{i}\right)$. Following the notes from the canonical representation approach (Lichtner 1985), $\chi_{i}$ is called the general component. However, $\chi_{i}$ does not have the straightforward physical meaning that the chemical components do.

Also denote

$$
\mathbf{R} \overrightarrow{\mathbf{W}}=\vec{\beta}=\left(\beta_{1}, \ldots, \beta_{N_{e}}, \beta_{N_{e}+1}, \ldots, \beta_{N_{e}+N_{k}}\right)^{T}
$$

and

$$
\overrightarrow{\mathbf{L}}(\vec{\chi})=\mathbf{Q}^{T} \overrightarrow{\mathbf{L}}(\overrightarrow{\mathbf{C}})
$$


Note the last $N_{k}$ components of $\vec{\beta}$ contain only the source terms of kinetic reactions.

Now Equation (3.43) can be written in three groups with different source terms. Group I $\left(\chi_{1}, \ldots, \chi_{N_{e}}\right)^{T}$ contains source terms of equilibrium and kinetic reactions:

$$
\frac{\partial}{\partial t}\left[\phi_{D} \rho_{\ell}\left(s_{g} K_{H}^{-1}+s_{\ell}\right) \chi_{l}\right]=L\left(\chi_{l}\right)+\beta_{l}, \quad l=1, \ldots, N_{e}
$$

Group II, kinetic reactive general components $\left(\chi_{N_{e}+1}, \ldots, \chi_{N_{e}+N_{k}}\right)^{T}$, contains the source terms only of the kinetic reactions:

$$
\frac{\partial}{\partial t}\left[\phi_{D} \rho_{\ell}\left(s_{g} K_{H}^{\prime-1}+s_{\ell}\right) \chi_{l}\right]=L\left(\chi_{l}\right)+\beta_{l}, \quad l=N_{e}+1, \ldots, N_{r}
$$

where $N_{r}=N_{e}+N_{k}$ is the total number of reactions. Group III, nonreactive general components, $\left(\chi_{N_{e}+N_{k}+1}, \ldots, \chi_{N_{s}}\right)^{T}$, does not contain the source terms of either type of reactions:

$$
\frac{\partial}{\partial t}\left[\phi_{D} \rho_{\ell}\left(s_{g} K_{H}^{\prime-1}+s_{\ell}\right) \chi_{l}\right]=L\left(\chi_{l}\right), \quad l=N_{r+1}, \ldots, N_{s}
$$

Because Equation (3.47) contains source terms of equilibrium reactions, it is still difficult to directly solve Equations (3.47) through (3.49) together. However, because of the equilibrium assumption made for the $N_{e}$ reactions Equation (3.47) is disregarded and replaced with the $N_{e}$ equilibrium conditions

$$
\prod_{i=1}^{N_{s}}\left(\gamma_{i} C_{i}\right)^{v_{i j}}=K_{j}^{e q}, j=1, \ldots, N_{e}
$$

Equations (3.48) through (3.50) are the working equations. They contain $N_{s}$ unknowns and $N_{s}$ equations; thus, this system of equations is closed.

The working equations contain only $N_{k}$ nonlinear PDE plus $N_{e}$ algebra equations and $N_{s}-N_{r}$ linear PDE. Compared with the canonical representation approach, that yields $N_{s}-N_{e}$ nonlinear PDE, and this approach yields $N_{s}-N_{e}-N_{k}$ fewer nonlinear PDE. At the extreme case where $N_{s}=N_{e}+N_{k}$, those two approaches yield the same number of nonlinear PDE. Reducing the number of nonlinear PDE can save computing time. The real savings depends on the ratio of $N_{s}$ and $N_{r}$.

\subsubsection{Finite Difference Discretization}

Two finite difference discretization schemes are used in STORM. For the flow and solute transport portions derived from STOMP, 3-D control volume discretization is used, but for the reactive transport portion derived from AREST-CT, a 2-D finite difference discretization is used. To demonstrate the discretization scheme used in the STOMP-derived portions of STORM, Equations (3.48) and (3.49) are 
discretized using the control-volume finite difference method on a rectangular 2-D domain (Strikwerda 1989). For notational convenience in the discretization procedure, the subscript $i$ is no longer used to denote different equations; rather, as by convention, the subscript is used to denote grid point and superscript to denote different time slices. Equation (3.48) can be discretized to

$$
\begin{aligned}
& \frac{\left\{\phi_{D} \rho_{\ell}\left(s_{g} K_{H}^{\prime-1}+s_{\ell}\right) \chi\right\}_{i, j}^{t+\delta t}-\left\{\phi_{D} \rho_{\ell}\left(s_{g} K_{H}^{\prime-1}+s_{\ell}\right) \chi\right\}_{i, j}^{t}}{\Delta t} \\
& =\lambda\left[\frac{\left\{\phi_{D} \rho_{\ell}\left(s_{g} \mathbf{V}_{g} K_{H}^{\prime-1}+s_{\ell} \mathbf{V}_{\ell}\right) \chi\right\}_{i, j}^{t+\delta t}-\left\{\phi_{D} \rho_{\ell}\left(s_{g} \mathbf{V}_{g} K_{H}^{\prime-1}+s_{\ell} \mathbf{V}_{\ell}\right) \chi\right\}_{i-1, j}^{t+\delta t}}{x_{i, j}-x_{i-1, j}}\right] \\
& +\lambda\left[\frac{\left\{\phi_{D} \rho_{\ell}\left(s_{g} \mathbf{V}_{g} K_{H}^{\prime-1}+s_{\ell} \mathbf{V}_{\ell}\right) \chi\right\}_{i, j}^{t+\delta t}-\left\{\phi_{D} \rho_{\ell}\left(s_{g} \mathbf{V}_{g} K_{H}^{\prime-1}+s_{\ell} \mathbf{V}_{\ell}\right) \chi\right\}_{i, j-1}^{t+\delta t}}{y_{i, j}-y_{i, j-1}}\right] \\
& +(1-\lambda)\left[\frac{\left\{\phi_{D} \rho_{\ell}\left(s_{g} \mathbf{V}_{g} K_{H}^{\prime-1}+s_{\ell} \mathbf{V}_{\ell}\right) \chi\right\}_{i, j}^{t}-\left\{\phi_{D} \rho_{\ell}\left(s_{g} \mathbf{V}_{g} K_{H}^{\prime-1}+s_{\ell} \mathbf{V}_{\ell}\right) \chi\right\}_{i-1, j}^{t}}{x_{i, j}-x_{i-1, j}}\right] \\
& +(1-\lambda)\left[\frac{\left\{\phi_{D} \rho_{\ell}\left(s_{g} \mathbf{V}_{g} K_{H}^{\prime-1}+s_{\ell} \mathbf{V}_{\ell}\right) \chi\right\}_{i, j}^{t}-\left\{\phi_{D} \rho_{\ell}\left(s_{g} \mathbf{V}_{g} K_{H}^{\prime-1}+s_{\ell} \mathbf{V}_{\ell}\right) \chi\right\}_{i, j-1}^{t}}{y_{i, j}-y_{i, j-1}}\right] \\
& +\lambda\left[\frac{\frac{\{\chi\}_{i+1, j}^{t+\delta t}-\{\chi\}_{i, j}^{t+\delta t}}{x_{i+1, j}-x_{i, j}}\left\{\phi_{D} \rho_{\ell}\left(s_{g} \mathbf{D}_{i}^{g} K_{H}^{\prime-1}+s_{\ell} \mathbf{D}_{i}^{\ell}\right)\right\}_{i+\frac{1}{2}, j}-\frac{\{\chi\}_{i-1, j}^{t+\delta t}-\{\chi\}_{i, j}^{t+\delta t}}{x_{i-1, j}-x_{i, j}}\left\{\phi_{D} \rho_{\ell}\left(s_{g} \mathbf{D}_{i}^{g} K_{H}^{\prime-1}+s_{\ell} \mathbf{D}_{i}^{\ell}\right)\right\}_{i-\frac{1}{2}, j}}{\left(x_{i+1, j}-x_{i-1, j}\right) / 2}\right] \\
& +(1-\lambda)\left[\frac{\frac{\{\chi\}_{i+1, j}^{t}-\{\chi\}_{i, j}^{t}}{x_{i+1, j}-x_{i, j}}\left\{\phi_{D} \rho_{\ell}\left(s_{g} \mathbf{D}_{i}^{g} K_{H}^{\prime-1}+s_{\ell} \mathbf{D}_{i}^{\ell}\right)\right\}_{i+\frac{1}{2}, j}-\frac{\{\chi\}_{i-1, j}^{t}-\{\chi\}_{i, j}^{t}}{x_{i-1, j}-x_{i, j}}\left\{\phi_{D} \rho_{\ell}\left(s_{g} \mathbf{D}_{i}^{g} K_{H}^{\prime-1}+s_{\ell} \mathbf{D}_{i}^{\ell}\right)\right\}_{i-\frac{1}{2}, j}}{\left(x_{i+1, j}-x_{i-1, j}\right) / 2}\right] \\
& +\lambda\left[\frac{\frac{\{\chi\}_{i, j+1}^{t+\delta t}-\{\chi\}_{i, j}^{t+\delta t}}{y_{i, j+1}-y_{i, j}}\left\{\phi_{D} \rho_{\ell}\left(s_{g} \mathbf{D}_{i}^{g} K_{H}^{\prime-1}+s_{\ell} \mathbf{D}_{i}^{\ell}\right)\right\}_{i, j+\frac{1}{2}}-\frac{\{\chi\}_{i, j-1}^{t+\delta t}-\{\chi\}_{i, j}^{t+\delta t}}{y_{i, j-1}-y_{i, j}}\left\{\phi_{D} \rho_{\ell}\left(s_{g} \mathbf{D}_{i}^{g} K_{H}^{\prime-1}+s_{\ell} \mathbf{D}_{i}^{\ell}\right)\right\}_{i, j-\frac{1}{2}}}{\left(y_{i, j+1}-y_{i, j-1}\right) / 2}\right]
\end{aligned}
$$




$$
\left.\begin{array}{l}
+(1-\lambda)\left[\frac{\frac{\{\chi\}_{i, j+1}^{t}-\{\chi\}_{i, j}^{t}}{y_{i, j+1}-y_{i, j}}\left\{\phi_{D} \rho_{\ell}\left(s_{g} \mathbf{D}_{i}^{g} K_{H}^{\prime-1}+s_{\ell} \mathbf{D}_{i}^{\ell}\right)\right\}_{i, j+\frac{1}{2}}-\frac{\{\chi\}_{i, j-1}^{t}-\{\chi\}_{i, j}^{t}}{y_{i, j-1}-y_{i, j}}\left\{\phi_{D} \rho_{\ell}\left(s_{g} \mathbf{D}_{i}^{g} K_{H}^{\prime-1}+s_{\ell} \mathbf{D}_{i}^{\ell}\right)\right\}_{i, j-\frac{1}{2}}}{\left(y_{i, j+1}-y_{i, j-1}\right) / 2}\right] \\
+\beta_{l}
\end{array}\right]
$$

where $\lambda$ is a time-weighting parameter: when $\lambda=1$, it is a fully implicit scheme, $\lambda=0.5$ is the Crank-Nicolson scheme; $\lambda=0$, is an explicit scheme. An upwinding scheme is used; thus,

$$
i^{\prime}=\left\{\begin{array}{l}
i+1, \text { when } \mathbf{V}_{\ell x}>0 \\
i, \text { when } \mathbf{V}_{\ell x}<0
\end{array} \text {, and } j^{\prime}=\left\{\begin{array}{l}
j+1, \text { when } \mathbf{V}_{\ell y}>0 \\
j, \text { when } \mathbf{V}_{\ell y}<0
\end{array}\right. \text {. }\right.
$$

Equation (3.49) can be discretized in the same manner. The only difference is that Equation (3.49) does not contain the $\beta$ term.

The main argument against upwinding is the associated numerical diffusion that smears reaction fronts. However, upwinding has the advantage of being less prone to numerical oscillation. As noted by (Yeh et al. 1993), "one scheme resolving the problems of numerical oscillation tends to yield excessive numerical spreading, whereas the other scheme resolving the problems of numerical spreading tends to create severe numerical oscillation."

\subsubsection{Fully Coupled Algorithm}

Equation (3.51) is a nonlinear equation that can be solved by the Newton-Raphson iteration that first converts Equation (3.51) to a linear equation (Press et al. 1986):

$$
\mathbf{A} \vec{\delta}^{k}=\mathbf{b}
$$

where $\mathbf{A}$ is the global Jacobian matrix, $\vec{\delta}^{k}$ is a correction on the previous solution $\vec{\chi}^{k}$, and $\mathbf{b}$ is the constant term. After solving Equation (3.52), corrections are made on $\vec{\chi}^{k}$ :

$$
\vec{\chi}^{k+1}=\vec{\chi}^{k}-\vec{\delta}^{k}
$$

The new solution $\vec{\chi}^{k+1}$ gives new values of $\mathbf{A}$ and $\mathbf{b}$ that in turn yield $\vec{\chi}^{k+2} \ldots$ and the iteration is continued until the solution meets convergence criteria.

The preceding algorithm solves the transport and reaction parts in one step. Such an algorithm is referred to as fully coupled, although other researchers have given it different names, such as one-step (Mangold and Tsang 1991), DAE (Yeh and Tripathi 1989), and global implicit (Steefel and Lasaga 1994). 
As stated by Yeh and Tripathi (1989), the difficulty of implementing a fully coupled algorithm is in the huge size of the global Jacobian matrix A. In their 2-D example with $60 \times 50$ grids and 30 species, the global Jacobian matrix A has $90,000 \times 90,000=8,100,000,000$ entries. Assuming each entry uses 32 bits (double precision), the matrix requires $2025 \mathrm{MB}$ of memory. It is impractical to store such a large matrix in most computers, and even matrix compression techniques cannot help much. However, the difficulty of storing $\mathbf{A}$ does not necessarily mean it is impractical to solve the equation. Many numerical algorithms used to solve Equation (3.52), for example, iteration schemes (Golub and Van Loan 1983), do not need to generate and store the global Jacobian matrix $\mathbf{A}$ in one piece.

Generally, in iteration algorithms, A is divided into many submatrixes, so during the iteration procedure only one submatrixes is needed. A natural way is to divide $\mathbf{A}$ into submatrixes with each submatrix containing the information of one node. The size of the submatrixes is $N_{s} \times N_{s}$. During the iteration, it is necessary only to solve a much smaller linear equation:

$$
\mathbf{A}_{i, i} \vec{\delta}_{i}=\mathbf{b}_{i}-\sum_{i \neq j} \mathbf{A}_{i, j} \vec{\delta}_{j}
$$

where $\mathbf{A}_{i, i}$ is the $i$ th submatrix in diagonal, while $\mathbf{A}_{i, j}$ is an off-diagonal submatrix.

This way of dividing $\mathbf{A}$ allows the solution of the problem on the entire domain, node by node. Two classical ways are known to iterate the node-by-node scheme: one is the Jacobi iteration and the other the Gauss-Seidel iteration (Press et al. 1986). The difference between the Jacobi and the Gauss-Seidel iterations is the latter uses the latest updated results of iteration, but the former does not. That difference gives the Gauss-Seidel iteration a factor of two in speed over the Jacobi iteration (Press et al. 1986). Therefore, Gauss-Seidel iteration is always preferred over Jacobi iteration.

Equation (3.54) involves a fully coupled solute transport and geochemical reactions, solved on a node-by-node basis using Gauss-Seidel iteration. This algorithm is hereafter referred to as the Fully Coupled algorithm. The size of the Jacobian matrix involved in the Fully Coupled algorithm is only $N_{s} \times N_{s}$. Even a system with 100 species can be handled easily by most computers. Moreover, the size of the matrix is independent of the number of nodes and thus independent of the dimensions of problems. The algorithm is applicable to 1-, 2-, or 3-D domains. Although the Fully Coupled algorithm bears the inherent disadvantage of slow convergence from Gauss-Seidel, it does not require large memory that otherwise prevents the problem from solution. Experience shows that combined with a flexible time-step control scheme, the algorithm can solve various practical problems.

\subsubsection{Operator Split Algorithm}

As an alternative to the Fully Coupled algorithm, an Operator Split algorithm to solve the reactiontransport equations is available in STORM. As its name suggests, the Operator Split algorithm splits the reaction-transport PDE into two parts. One is the transport part that is a linear PDE; the other is the chemical reaction part that is a nonlinear ordinary differential equation (ODE). The algorithm then solves them separately and sequentially (Mangold and Tsang 1991). It has been proved the Operator Split algorithm has an inherent mass conservation error (Valocchi and Malmstead 1992). Although a remediation scheme has been proposed by these authors to address that problem, the scheme works only 
when the boundary condition is homogenous (Barry et al. 1996). Otherwise, it can introduce an even larger error. Another remedial method is to iterate the two steps until they converge (Yeh and Tripathi 1991). This latter approach is implemented in STORM.

In the Operator Split algorithm, by splitting the transport part from the reaction part, the solute transport mass conservation equation is decoupled and thus can be solved separately. In STORM, when using the Operator Split algorithm, the solute transport mass conservation equation is solved as described in Section 3.3. The advantage is that each discretized PDE is a linear equation where the dimension of the coefficient matrix, $\mathbf{b}$, is equal to the number of nodes squared. Therefore, $\mathbf{b}$ is $\left(N_{s}\right)^{2}$ times smaller than the global matrix A. For the preceding example, it is 9,000,000. Combined with matrix compression techniques, the matrix can be stored in workstations with moderate memory. Because $\mathbf{b}$ can be generated and stored at once, more sophisticated techniques of solving linear equations (such as conjugate gradient or preconditioning conjugate gradient methods) can be used to accelerate convergence.

The second step of Operator Split algorithm is to solve the nonlinear ODE at each node to obtain reaction rates for each species. This step is similar to the Fully Coupled algorithm. However, unlike the Fully Coupled algorithm where the latest updated results of neighboring nodes are used, the Operator Split algorithm cannot use them because the latest updated results of neighboring nodes cannot propagate to the current node until the next iteration between the first step and the second step. The solution of the geochemical reaction rates, while using the Operator Split algorithm, then effectively uses Jacobi iteration. Therefore, as described in Section 3.4.4, its convergence speed is only half that of the Fully Coupled algorithm.

Although the Operator Split algorithm is slower than the Fully Coupled algorithm, it is well known that contaminant transport simulators based on single-point upstream-weighting for solution of the conservation equations (Section 3.4.3) suffer from a grid size dependent artificial diffusion that smears otherwise sharp fronts. To enable STORM to better manage advection-dominated problems, such as simulating the pressurized unsaturated flow (PUF) experiments, a total variation diminishing (TVD) transport scheme has been implemented in conjunction with the Operator Split algorithm.

A flux-limited third-order scheme is applied to the advective-diffusion equations in STORM following a method that is shown to exhibit close agreement with analytical solutions (Gupta et al. 1991). The difference scheme for the conservation equation is illustrated for 1-D, strictly advective flow with constant grid size. This arrangement results in a strictly hyperbolic conservation equation:

$$
\frac{\partial c}{\partial t}+u \frac{\partial c}{\partial x}=0
$$

where $c$ is the concentration of the aqueous species, $t$ is time, $x$ is distance, and $u$ is the velocity (taken to be a positive constant). Integrating over mesh cell $i$ and time step interval $[n, n+1]$ yields

$$
\frac{c_{i}^{n+1}-c_{i}^{n}}{\Delta t}=\frac{u \bar{c}_{i+1 / 2}-u \bar{c}_{i-1 / 2}}{\Delta x}=0
$$


where $u \bar{c}_{i+1 / 2}$ represents the time averaged flux through the right edge of the $i$ th mesh cell. The principal problem in the formulation of difference schemes lies in the evaluation of these time-averaged fluxes. The finite-difference approximation may be generalized as

$$
\bar{c}_{i+1 / 2}=c_{i}^{n}+\frac{1}{2}(1-\lambda) F_{i} \Delta x
$$

where $\lambda$ is the Courant number and $F_{i}$ is the concentration gradient of the upwind cell. For $F_{i}=0$, Equation (3.57) reduces to single-point upstream weighting, the technique used in the Fully Coupled algorithm that is only first-order accurate and consequently highly diffusive. A third-order scheme provides higher accuracy (Gupta et al. 1991)

$$
F_{i}=\frac{2-\lambda}{3} \frac{c_{i+1}^{n}-c_{i}^{n}}{\Delta x}+\frac{1+\lambda}{3} \frac{c_{i}^{n}-c_{i-1}^{n}}{\Delta x}
$$

Although higher-order schemes give better resolution for discontinuities, they exhibit spurious oscillations around these points. The underlying principle of constructing a TVD scheme is to combine lower- and higher-order fluxes and impose limiting functions on the higher-order flux to prevent formation of local extrema (Harten 1983). The higher-order flux is viewed as consisting of the first-order flux plus a corrective term. Equation (3.57) can be rewritten in the following terms:

$$
\bar{c}_{i+1 / 2}=c_{i}^{n}+\phi(r) \frac{c_{i+1}^{n}-c_{i}^{n}}{2}(1-\lambda)
$$

where $\phi(r)$ is the flux-limiting function and $\mathrm{r}$ is a measure of the smoothness of the data. One possible choice for $r$ is the ratio of consecutive gradients (Sweby 1984). For $u>0$,

$$
r=\frac{c_{i}^{n}-c_{i-1}^{n}}{c_{i+1}^{n}-c_{i}^{n}}
$$

The third-order scheme can now be obtained by defining $\phi(r)$ appropriately:

$$
\phi(r)=\frac{2-\lambda}{3}+\frac{1+\lambda}{3} r
$$

To prevent non-physical oscillations, the limiting function $\phi(r)$ must be chosen so that limited antidiffusive flux is maximized in amplitude subject to the constraint of the resulting scheme being TVD that requires the following equation (Sweby 1984):

$$
0 \leq \frac{\phi(r)}{r}, \phi(r) \leq 2
$$


Equation (3.62) results in the following TVD limiting function for the third-order scheme:

$$
\phi(r)=\operatorname{MAX}[0, \operatorname{MIN}(2,2 \mathrm{r}, \phi(r))]
$$

For multidimensional flow problems, the difference scheme given by Equation (3.59) can be applied similarly in the $\mathrm{y}$ and $\mathrm{z}$ directions.

\subsection{Boundary Conditions}

The discretization methods described previously for the mass, energy, and solute mass conservation equations strictly applied to interior nodes (that is, nodes surrounded by neighboring nodes). For nodes adjacent to a domain boundary or an inactive node, the discretized forms of the governing equations are modified according to the user-specified boundary conditions. Zero flux boundary conditions are applied whenever no boundary condition is specified for a boundary surface. Boundary conditions that can be specified for a boundary surface are varied depending on the operational mode and other associated boundary conditions.

Eight boundary conditions are appropriate for flow boundaries and applicable to the conservation equations for water and air: Dirichlet, Neumann, zero flux, initial condition, saturated, unit gradient, hydraulic gradient, and free gradient. The Dirichlet boundary condition specifies the value of intrinsic properties on the boundary surfaces (for example, aqueous pressure and gas pressure). The Neumann boundary condition specifies a surface flux on the boundary surface (for example, aqueous Darcy velocity and gas Darcy velocity). The zero flux boundary condition specifies an impermeable boundary for flow or transport and serves as the default condition for undeclared boundary surfaces (for example, zero fluid flow). The initial condition boundary condition uses the initial conditions in the nodes adjacent to a boundary surface to fix the intrinsic properties on the boundary surface. The saturated boundary condition is appropriate for fluid flow boundaries for two-phase systems and establishes zero capillary pressure conditions on the boundary surface. The unit gradient boundary condition is appropriate for fluid flow boundaries and establishes a fluid pressure on the boundary surface equal to that at the adjacent node modified by the hydraulic gradient for the fluid. The free gradient condition is appropriate for fluid flow boundaries for two-phase systems and establishes a fluid pressure on the boundary surface by extrapolating the local pressure gradient within the computational domain to the boundary surface. The hydraulic gradient boundary condition is appropriate for fluid flow boundaries and establishes a series of boundary pressures according to the local hydraulic gradient for the fluid.

The five boundary conditions appropriate for transport boundaries and applicable to the conservation equations for energy and solute mass follow: Dirichlet, zero flux, initial condition, outflow, and inflow. The Dirichlet boundary condition specifies the value of intrinsic properties on the boundary surfaces (for example, temperature or solute concentration). The zero flux boundary condition specifies an impermeable boundary for flow or transport and serves as the default condition for undeclared boundary surfaces (e.g., zero heat flux or zero solute flux). The initial condition boundary condition uses the initial conditions in the nodes adjacent to a boundary surface to fix the intrinsic properties on the boundary surface. The outflow boundary condition considers transport out of the computational domain by advection only, so no diffusive transport occurs. This boundary condition will not transport energy or solute mass into the domain. The inflow boundary condition considers transport into the computational 
domain only by advection and no diffusive transport occurs. This boundary condition will not transport energy or solute mass out of the domain.

In general, boundary conditions for simulations that involve the solution of multiple governing equations can be combined in a variety of ways, with one major restriction. Specification of a Neumann boundary condition for the energy equation on a boundary surface (other than the zero flux condition) requires that flow equation boundary conditions for that boundary surface be specified as zero flux. Nonzero fluid flow and heat transport can be achieved for boundary nodes through a combination of flow boundary conditions, a zero flux energy boundary, and energy sources for the boundary nodes. Regardless of the boundary condition or combination of boundary conditions a sufficient number of independent intensive variables must be declared to specify the thermodynamic and hydrologic state on the boundary surface. Definitions for geometric parameters for nodes with boundary surfaces on the west side are shown in Figure 3.3 for the $\mathrm{X}-\mathrm{Z}$ Cartesian coordinate plane and in Figure 3.4 for the $\mathrm{X}-\mathrm{Y}$ Cartesian coordinate plane.

\subsubsection{Dirichlet Boundary Condition}

The Dirichlet boundary condition is equivalent to specifying the value for the primary unknown on the boundary surface. Assigned values of primary variables are used to compute secondary variables for the boundary surface. Average properties for transport between a boundary surface and the adjacent node are computed with user-specified averaging schemes between the values of the properties on the boundary surface and adjacent node. For example, the discretized forms for Darcy mass flux and diffusive mass flux rates of water, Equation (3.6), are modified for a Dirichlet aqueous phase boundary condition on a west boundary surface, according to Equations (3.64) and (3.65), respectively. For the solute mass conservation equation, a Dirichlet boundary condition on the west surface produces a modification to the discretized conservation equation, Equation (3.26), according to Equation (3.66).

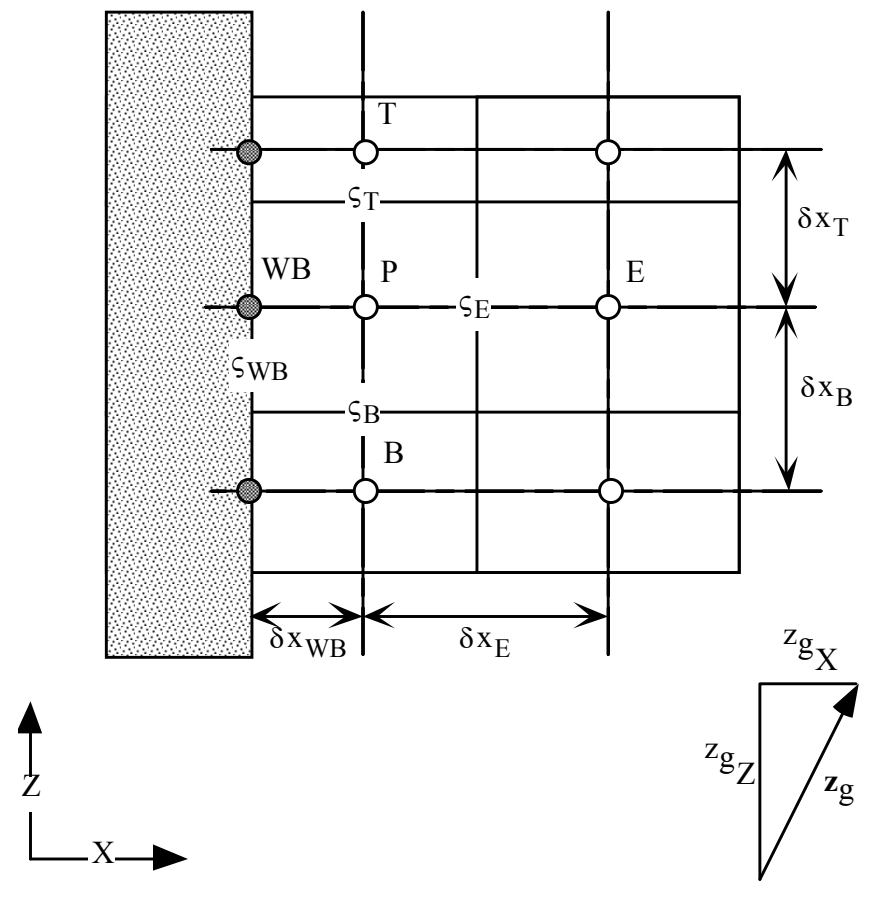


Figure 3.3. $X-Z$ Cartesian Coordinate Plane for West Boundary
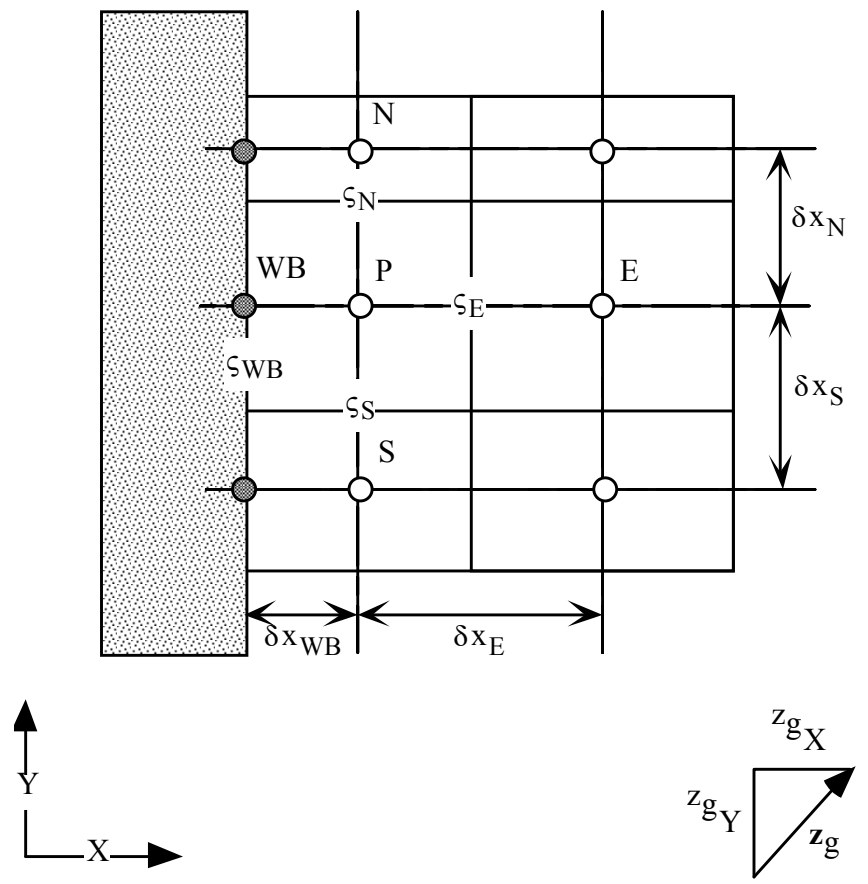

Figure 3.4. $\mathrm{X}-\mathrm{Y}$ Cartesian Coordinate Plane for West Boundary

$$
\begin{gathered}
F_{l W B}^{w}=-\frac{\left\langle\omega_{l}^{w} \rho_{l} k_{r l}\right\rangle_{W B}^{u w}\langle\mathbf{k}\rangle_{W B}^{h}}{\left\langle\mu_{l}\right\rangle_{W B}^{h}}\left(\frac{\left(P_{l P}-P_{l W B}\right)}{\delta x_{W B}}+\left\langle\rho_{l} g\right\rangle_{W B}^{a} z_{g W B}\right) \\
J_{l_{W B}^{w}}^{w}=-\left\langle\tau_{l} n_{D} \rho_{l} s_{l} \frac{M^{w}}{M_{l}} \mathbf{D}_{l}^{w}\right\rangle_{W B}^{h} \frac{\left(\chi_{l P}^{w}-\chi_{l W B}^{w}\right)}{\delta x_{W B}} \\
a_{P} C_{P}-\sum_{\varsigma=E, S, N, B, T} a_{\varsigma} C_{\varsigma}=\frac{\partial C}{\partial t} \bar{V}-\dot{m}^{C} \bar{V}-\dot{R}^{C} C \bar{V}+a_{W B} C_{W B}
\end{gathered}
$$

\subsubsection{Neumann Boundary Condition}

The Neumann boundary condition is equivalent to specifying the flux on a boundary surface. Fluxes that may be specified are aqueous volumetric, gas volumetric, energy, and solute mass. For example, the discretized form for Darcy mass flux of water, Equation (3.6), is modified for a Neumann boundary condition on a west boundary surface, according to Equation (3.67). Sufficient information is needed to fix the thermodynamic and hydrologic state on the boundary surface. Calculation of phase pressures from phase volumetric flow rates requires an iterative solution because averaged values of properties (for 
example, relative permeability) are nonlinear functions of the phase pressure on the boundary. To avoid an iterative solution of the phase pressure on the boundary phase, pressures are computed assuming a unit relative permeability, according to Equation (3.68) for a west boundary surface.

$$
\begin{gathered}
F_{l W B}^{w}=\left\langle\omega_{l}^{w} \rho_{l}\right\rangle_{W B}^{u w} V_{l W B} \\
P_{l W B}=\delta x_{W B}\left(\frac{V_{l W B}^{w}\left\langle\mu_{l}\right\rangle_{W B}^{h}}{\langle\mathbf{k}\rangle_{W B}^{h}}+\left\langle\rho_{l} g\right\rangle_{W B}^{a} z_{g W B}\right)+P_{l P}
\end{gathered}
$$

\subsubsection{Zero Flux Boundary Condition}

The zero flux boundary condition is the default boundary condition and is equivalent to specifying zero flow or transport across the boundary surface. Complex boundary conditions that involve multiple specifications on a single boundary surface require enough information to be specified to fix the thermodynamic and hydrologic state on the boundary surface. For example, a recognized boundary condition combination for a water-air system (that is, the Two-Phase Volatile operational mode) is a Dirichlet aqueous boundary and zero flux gas boundary. Isothermal water-air systems require two independent intensive variables to fix the thermodynamic and hydrologic state of the system. For this boundary system, the Dirichlet aqueous boundary assigns a value to the aqueous pressure. The gas pressure can be specified by user input or be computed using zero flux boundary conditions and solving Equation (3.6) for the gas pressure, according to Equation (3.69) for a west boundary surface.

$$
P_{g W B}=\delta x_{W B}\left\langle\rho_{g} g\right\rangle_{W B}^{a} z_{g W B}+P_{g P}
$$

\subsubsection{Initial Condition Boundary Condition}

The initial condition boundary condition is identical in application to the Dirichlet boundary condition with the following exception: the primary variable is fixed to the initial value at the adjacent node. No user input is required for this type of boundary condition because boundary values are obtained through the initial condition specifications. Initial boundary pressures are computed once at the start of a simulation.

\subsubsection{Saturated Boundary Condition}

The saturated boundary condition is recognized for water-air systems and performs as do dynamic Dirichlet boundary conditions, where zero capillary pressure is maintained on the boundary surface. The saturated boundary condition fixes the aqueous pressure equal to the gas pressure on a boundary surface, regardless of the boundary condition for the gas pressure. For a single-phase system, the gas pressure is fixed through the initial conditions and the aqueous pressure is maintained on a saturated boundary equal to this gas pressure. For a two-phase system, the gas pressure on a boundary surface is user-specified according to the gas-phase boundary conditions. The saturated boundary condition for the aqueous phase 
fixes the aqueous pressure at the boundary surface equal to the gas pressure. Saturated boundary pressures are computed with each Newton-Raphson iteration.

\subsubsection{Unit Gradient Boundary Condition}

The unit gradient boundary condition is recognized for aqueous-phase and gas-phase boundary conditions and maintains a unit gradient in the phase hydraulic head. A unit gradient in the phase hydraulic head is equivalent to setting the normalized Darcy velocity equal to minus one, according to Equation (3.70) for a west boundary surface. Phase pressure on the boundary surface is computed by solving Equation (3.70) for the boundary pressure according to Equation (3.71) for a west boundary surface. Unit gradient boundary pressures are computed with each Newton-Raphson iteration.

$$
\begin{gathered}
\frac{V_{\gamma W B}\left\langle\mu_{\gamma}\right\rangle_{W B}^{h}}{\left\langle k_{r \gamma}\right\rangle_{W B}^{u w}\langle\mathbf{k}\rangle_{W B}^{h}}=-1=-\left(\frac{\left(P_{\gamma P}-P_{\gamma W B}\right)}{\delta x_{W B}}+\left\langle\rho_{\gamma} g\right\rangle_{W B}^{a} z_{g W B}\right) \text { for } \gamma=l, g \\
P_{\gamma W B}=P_{\gamma P}+\delta x_{W B}\left(\left\langle\rho_{\gamma} g\right\rangle_{W B}^{a} z_{g W B}\right) \text { for } \gamma=l, g
\end{gathered}
$$

\subsubsection{Free Gradient Boundary Condition}

The free gradient boundary condition is recognized for aqueous-phase and gas-phase boundary conditions. This boundary condition is essentially a dynamic Dirichlet-type boundary, where the pressure on the boundary surface is set to maintain the pressure gradient in the interior nodes adjacent to the boundary surface. This boundary type requires, as a minimum, two active nodes adjacent to the boundary surface. The gradient in phase pressure is linearly extrapolated from the interior nodes to the boundary surface to determine the boundary pressure. Free gradient boundary pressures are computed with each Newton-Raphson iteration.

\subsubsection{Outflow Boundary Condition}

The outflow boundary condition is recognized for transport boundaries (for example, energy transport, solute transport). This boundary condition allows transported quantities to be moved across a boundary surface by advection only. Inward transport by diffusion or transport across the boundary surface is prohibited. Energy flux across an outflow boundary surface on a west boundary is computed according to Equation (3.72). Solute flux across an outflow boundary surface on a west boundary is computed according to Equation (3.73).

$$
\begin{gathered}
Q_{\gamma W B}=-\left(\rho_{\gamma} h_{\gamma}\right)_{P} \max \left[-V_{\gamma W B}, 0\right] \text { for } \gamma=l, g \\
G_{\gamma_{W B}}^{C}=-C_{\gamma P} \max \left[-V_{\gamma W B}, 0\right] \text { for } \gamma=l, g
\end{gathered}
$$




\subsubsection{Inflow Boundary Condition}

The inflow boundary condition is recognized for transport boundaries (for example, energy transport and solute transport). This boundary condition allows transported quantities to be inwardly transported across a boundary surface by advection only. Transport by diffusion or transport outward across the boundary surface is prohibited. Energy flux across an inflow boundary surface on a west boundary is computed according to Equation (3.74). Solute flux across an inflow boundary surface on a west boundary is computed according to Equation (3.75).

$$
\begin{gathered}
Q_{\gamma W B}=\left(\rho_{\gamma} h_{\gamma}\right)_{P} \max \left[V_{\gamma W B}, 0\right] \text { for } \gamma=l, g \\
G_{\gamma_{W B}}^{C}=C_{\gamma P} \max \left[V_{\gamma W B}, 0\right] \text { for } \gamma=l, g
\end{gathered}
$$

\subsection{Time Steps}

Time step size may be limited by advection based on the Courant condition (3.76) and by diffusion/dispersion based on the von Neumann condition (3.77):

$$
\begin{gathered}
(\Delta t)_{A}=\frac{\Delta x}{v} \\
(\Delta t)_{D}=\frac{(\Delta x)^{2}}{3 D_{L}}
\end{gathered}
$$

where $(\Delta t)_{A}$ is the advective time step, $(\Delta t)_{D}$ is the diffusive time step, $\Delta x$ is the grid spacing, and $D_{L}$ is the longitudinal hydrodynamic dispersion coefficient.

\subsection{Parallel Processing}

STORM runs on parallel processors using a domain-decomposition approach (Dowd and Severance 1998). STORM uses the message-passing interface (MPI) for communication between parallel processes. MPI is a message-passing environment that is available on a wide range of hardware environments (http://www.netlib.org/mpi). Initially, the code determines the number of processors it should run on and creates copies of itself to run on each processor. Each copy of the running code (process) is given a unique process ID number starting with zero. Each process knows that the domain will be divided evenly among the processors and determines which subdomain to solve based on its process ID. At each iteration, each process exchanges left, right, lower and upper subdomain boundary values (ghost values) with its adjacent neighbors. The interprocess communication for a $200 \times 200$ node domain, divided into four subdomains, is shown in Figure 3.5. At each iteration, each process exchanges convergence information so that all processes will take the same number of iterations and reduce the time step if necessary. Each process has its own unique input and output files, identified by its process ID. 


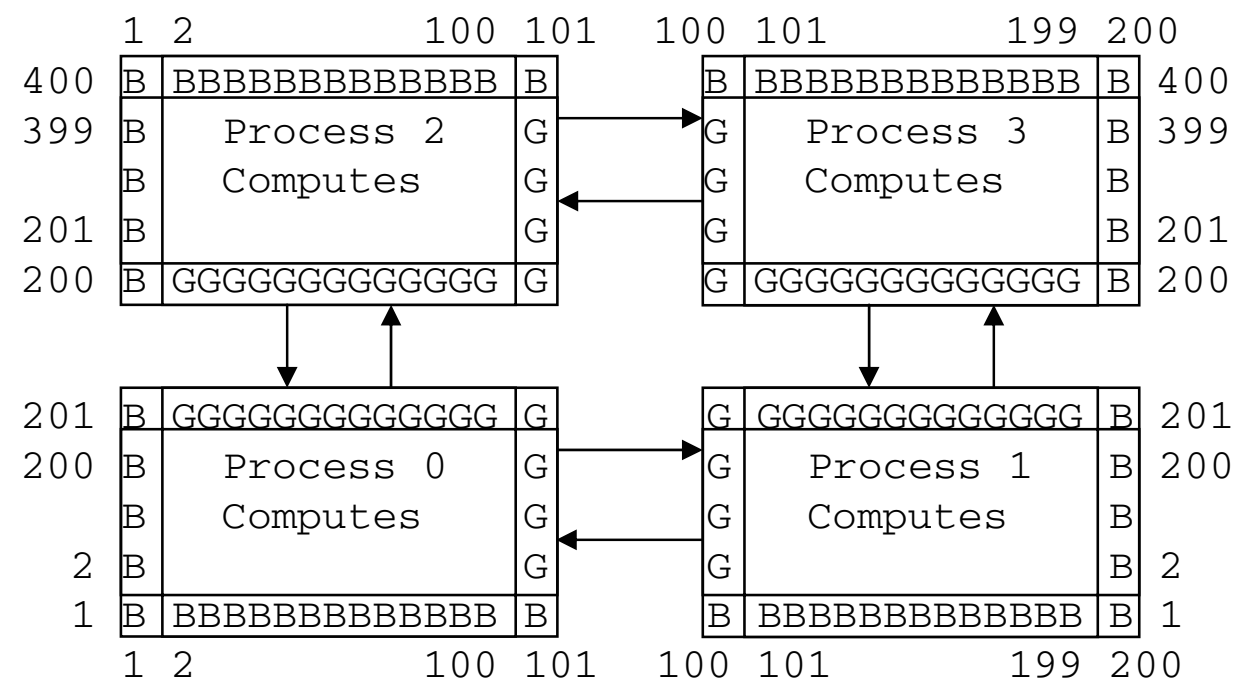

G Ghost values

Transferred with each time step
B Boundary values

Defined at each time step

Figure 3.5. Pattern of Communication Between Parallel Processes 


\subsection{Input File}

The STORM simulator is controlled through a text file that must be named input for proper execution. This input file has a structured format composed of cards that contain associated groups of input data. Depending on the operational mode, input cards might be required, optional, or unused. Required cards must be present in an input file. Optional cards are not strictly required to execute the simulator but may be required to execute a particular simulation. Unused cards are treated as additional text that is not recognized by the simulator but does not hinder proper execution. Cards may appear in any order within the input file; however, the data structure within a card is critical and must follow the formatting directives shown in Appendix A. Data structures within cards vary with the operational mode, which requires the user to follow a series of logic-type statements to construct a readable input file. The STORM simulator contains logic to capture and report input errors. These capabilities are primarily limited to indicating syntax or formatting errors and generally do not reveal errors like those associated with ill-posed problems, atypical parameters, or inappropriate grid structures, for example. Because of its text format, STORM input files are generated with text editors, word processors, spreadsheet programs, or graphical interfaces and are portable between computing environments. The input is case insensitive and allows considerable flexibility in specifying the simulation directives; however, close attention to formatting instructions in this section is necessary to prepare an executable input file.

\subsection{Input File Structure}

The number of required cards depends on the operational mode. If an attempt is made to execute the STORM simulator on an input file with an incomplete set of required cards, an error message will be generated and the code execution will stop. Optional cards are used to specify the STORM capabilities that may be required to execute a particular problem or generate desired output data. These cards are considered optional, because the capabilities accessed through these cards are not necessarily required to execute the code. Execution of the STORM simulator on input files with an incomplete set of optional cards yields messages that note the missing optional cards and allow the execution to continue. A summary of the required and optional cards that compose a STORM input file for each operational mode is shown in Table 4.1.

Each card is delimited on the first line with a card header that must contain a tilde symbol (for example, $\sim$ Simulation Title Card) as the character in the first column of the line. Cards may be arranged in any order within an input file; however, the input format within a card is generally structured. If a card type is repeated within an input file, the STORM simulator reads only the first card. Blank lines or additional comment lines can be included in the input file outside of the card structures. 
Table 4.1. Required and Optional Input Cards

\begin{tabular}{|c|c|c|}
\hline Operational Mode & Card Name & Card Status \\
\hline \multirow[t]{18}{*}{ All } & Initial Conditions & Optional \\
\hline & Boundary Conditions & Optional \\
\hline & Source & Optional \\
\hline & Output Control & Optional \\
\hline & Surface Flux & Optional \\
\hline & Reactive Transport Control & Required \\
\hline & Aqueous Species & Required \\
\hline & Gas Species & Required \\
\hline & Components & Required \\
\hline & Solid Species & Required \\
\hline & Fast Reactions & Required \\
\hline & Kinetic Aqueous Reactions & Required \\
\hline & Slow Reactions & Required \\
\hline & Fluid Decay & Required \\
\hline & Solid Decay & Required \\
\hline & Lithology & Required \\
\hline & Reaction BC & Required \\
\hline & Reaction IC & Required \\
\hline \multirow[t]{8}{*}{ Water } & Simulation Title & Required \\
\hline & Solution Control & Required \\
\hline & Grid & Required \\
\hline & Rock/Soil Zonation & Required \\
\hline & Mechanical Properties & Required \\
\hline & Hydraulic Properties & Required \\
\hline & Saturation Function & Required \\
\hline & Aqueous Relative Permeability Function & Required \\
\hline \multirow[t]{9}{*}{ Water-Air } & Simulation Title & Required \\
\hline & Solution Control & Required \\
\hline & Grid & Required \\
\hline & Rock/Soil Zonation & Required \\
\hline & Mechanical Properties & Required \\
\hline & Hydraulic Properties & Required \\
\hline & Saturation Function & Required \\
\hline & Aqueous Relative Permeability Function & Required \\
\hline & Gas Relative Permeability Function & Required \\
\hline \multirow[t]{9}{*}{ Water-Air-Energy } & Simulation Title & Required \\
\hline & Solution Control & Required \\
\hline & Grid & Required \\
\hline & Rock/Soil Zonation & Required \\
\hline & Mechanical Properties & Required \\
\hline & Hydraulic Properties & Required \\
\hline & Thermal Properties & Required \\
\hline & Saturation Function & Required \\
\hline & Aqueous Relative Permeability Function & Required \\
\hline
\end{tabular}




\subsection{Formatting and Notation}

Each input card has formatting specifications that must be followed to create an input file readable by the STORM simulator. The format structure of certain cards varies with the operational mode or other input options, and the user must strictly follow the formatting instructions for the particular operational mode of interest. The complexity of the input increases with the operational mode: mode 1 requires input for single-phase unsaturated flow, mode 2 requires input for two-phase flow, and mode 3 requires input for nonisothermal two-phase flow. Guides for formatting input cards and card lines are presented in Appendix A. The formatting guides shown in this appendix were written in a compact format using special notations. Definitions of the formatting notations used in Appendix A are given in Table 4.2.

Input files are organized into three hierarchical structures, cards, lines, and data. Cards are delimited by a tilde $(\sim)$ in the first column of the card title. Lines are delimited by hard returns and commas delimit data. The STORM simulator recognizes three different types of data, integers, real data, and character strings. Integers are used primarily to indicate indexes or integral numbers. Integer data must be entered without decimal points or exponential notation. Real data are used to indicate dimensional parameters and can contain decimal points or exponential notation. Character string data are primarily used to indicate names, options, and units and are limited to 64 characters unless otherwise noted. Card lines comprise a series of input data items delimited by commas. A comma at the end of the card line is required to close the last data item. The format structure for each line in a card is shown in Appendix A using a format guide (for example, Format: Integer ${ }^{a}$, Real $^{b}$, Char ${ }^{c}$,). Data types in the format statements are indicated as Integer, Real and Char (that is, integer, real, and character string) with lowercase letter superscripts. The lettered superscripts are used to correlate the data item with its position in the input line. The total length of a single input line cannot exceed 132 characters. Additional text may appear after the closing comma of an input line. Many input variables contain default values (indicated with underlining in Appendix A). Using a null entry accesses a default value. The null entry requires the closing comma for the input data item to immediately follow the previous closing comma or that only blank spaces fill the space between the closing commas. To start a line with a null entry, the closing comma can occur in column 1 or after a number of blank spaces. Example input cards are shown in Appendix A after the formatting instructions for each card. A considerable portion of the input file formatting and creation work is eliminated by developing input files from previously generated files or through an input generator.

Table 4.2. Input Format Notation Guide

\begin{tabular}{|l|l||}
\hline \multicolumn{1}{|c|}{ Notation } & \multicolumn{1}{|c|}{ Description } \\
\hline$\{$ Option $\}$ & $\begin{array}{l}\text { Enclosing braces indicate character string options. Options are } \\
\text { chosen by entering word(s) within the braces, exactly as shown. } \\
\text { Only one option should be chosen for each data entry. }\end{array}$ \\
\hline [ Optional ] & $\begin{array}{l}\text { Enclosing brackets indicate optional characters or words. These } \\
\text { characters can be entered to include the input file to improve its } \\
\text { readability. }\end{array}$ \\
\hline$\{\{$ Fractured $\}\}$ & $\begin{array}{l}\text { Indicates the word fractured is within the rock/soil type name, thus } \\
\text { indicating a dual-porosity model. }\end{array}$ \\
\hline$<$ Data Types $>$ & Indicates repeated formatting. \\
\hline Char ${ }^{\mathrm{a}}$ & Character string data type, referenced by superscript a. \\
\hline
\end{tabular}


Table 4.2. Input Format Notation Guide (contd)

\begin{tabular}{|c|c|}
\hline Notation & Description \\
\hline Integer $^{\mathrm{a}}$ & $\begin{array}{l}\text { Integer data type (no character data or decimal points) reference by } \\
\text { superscript a. }\end{array}$ \\
\hline Real $^{\mathrm{a}}$ & $\begin{array}{l}\text { Real data type (decimal points and exponential notation are } \\
\text { acceptable), reference by superscript a. }\end{array}$ \\
\hline \# & $\begin{array}{l}\text { A pound symbol in the first column indicates a comment line that is } \\
\text { ignored during execution. Comment lines may be placed inside or } \\
\text { outside card structures. All lines outside of the card structures are } \\
\text { ignored during execution. }\end{array}$ \\
\hline$\sim$ Card Name & A tilde symbol in the first column indicates the start of a new card. \\
\hline , & $\begin{array}{l}\text { Data entries are comma delimited. Commas shown in the line } \\
\text { format structures must be entered as shown, including a closing } \\
\text { comma at the end of each line. Characters following the last comma } \\
\text { of a data line are ignored during execution. }\end{array}$ \\
\hline $\operatorname{Units}^{\mathrm{a}}(\mathrm{m})$ & $\begin{array}{l}\text { Indicates the SI unit for the input data item referenced by superscript } \\
\text { a. }\end{array}$ \\
\hline Format: & $\begin{array}{l}\text { Indicates line formatting instructions and the beginning of a new } \\
\text { input line. Each format statement requires a new input line. }\end{array}$ \\
\hline Endcard: & Indicates end of a card. \\
\hline $\begin{array}{l}\text { For: Integer } \\
\text { Instructions } \\
\text { Endfor: Integer }\end{array}$ & Indicates instruction looping. \\
\hline $\begin{array}{l}\text { If: Name: Card }=\left\{\mathrm{Opt}_{-} 1\right\} \\
\quad \text { Instructions1 } \\
\text { Elseif: Name: Card }=\left\{\mathrm{Opt}_{-} 2\right\} \\
\quad \text { Instructions } 2 \\
\text { Elseif: } \\
\quad \text { Instructions } 3 \\
\text { Endif: }\end{array}$ & Indicates decision logic. \\
\hline $\begin{array}{l}\text { IfDef: Opt_1 } \\
\text { Instructions1 } \\
\text { ElseifDef: Opt_2 } \\
\text { Instructions2 } \\
\text { ElseDef: } \\
\text { Instructions3 } \\
\text { EndifDef: }\end{array}$ & Indicates $\mathrm{C}$ preprocessor options and logic. \\
\hline Note: & Indicates formatting information. \\
\hline
\end{tabular}

\subsection{Units}

The STORM simulator offers considerable flexibility in specifying units. The user can declare units for both input and output data. Unspecified units are assumed to be in standard Systeme Internationale (SI) units for the data item. The STORM simulator operates internally almost exclusively in SI units, 
with pressures expressed in gauge relative to 1 atmosphere. Unless specifically stated, all input and output pressures are expressed in absolute values. Unit variables are read by the STORM simulator as character strings, translated into primary unit form, and compared against the standard unit form for each data item. During the translation to primary unit form, a conversion factor to SI units is generated. A unit character string comprises a combination of the recognized units delimited by spaces or a single divisor symbol (that is, /). Only one divisor may appear in a unit character string. Spaces should not be used to separate the units immediately prior to or following the divisor symbol. The unit strings prior to the divisor symbol are considered part of the numerator and, conversely, the unit strings following the divisor symbol are considered part of the denominator. Units recognized by the STORM simulator are listed in Table 4.3.

Table 4.3. Recognized Units

\begin{tabular}{|c|c|c|c|c|}
\hline Notation & Description & SI Equivalent & Conversion to SI & Base Units \\
\hline 1 & one & N/A & $\mathrm{N} / \mathrm{A}$ & $\mathrm{N} / \mathrm{A}$ \\
\hline $\mathrm{aqu}$ & aqueous phase & N/A & N/A & $\mathrm{N} / \mathrm{A}$ \\
\hline aqueous & aqueous phase & $\mathrm{N} / \mathrm{A}$ & $\mathrm{N} / \mathrm{A}$ & N/A \\
\hline atm & atmosphere & $\mathrm{Pa}$ & 101325.0 & $\mathrm{M} / \mathrm{L} \mathrm{T}^{2}$ \\
\hline bar & bar & $\mathrm{Pa}$ & $1 . \times 10^{5}$ & $\mathrm{M} / \mathrm{L} \mathrm{T}^{2}$ \\
\hline btu & Btu & $\mathrm{J}$ & 1054.4 & $\mathrm{M} \mathrm{L}^{2} / \mathrm{T}^{2}$ \\
\hline $\mathrm{c}$ & Celsius & $\mathrm{C}$ & 1. & $\mathrm{~K}$ \\
\hline cal & calorie & $\mathrm{J}$ & 4.184 & $\mathrm{M} \mathrm{L}^{2} / \mathrm{T}^{2}$ \\
\hline ci & Curie & $\mathrm{N} / \mathrm{A}$ & $\mathrm{N} / \mathrm{A}$ & N/A \\
\hline $\mathrm{cm}$ & centimeter & $\mathrm{m}$ & $1 . \times 10^{-2}$ & $\mathrm{M}$ \\
\hline $\mathrm{cp}$ & centipoise & $\mathrm{Pa} \mathrm{s}$ & $1 . \times 10^{-3}$ & $\mathrm{M} / \mathrm{L} \mathrm{T}$ \\
\hline $\mathrm{d}$ & day & $\mathrm{s}$ & $1 / 86400$ & $\mathrm{~T}$ \\
\hline darcy & Darcy & $\mathrm{m}^{2}$ & $9.8697 \times 10^{-13}$ & $\mathrm{~L}^{2}$ \\
\hline day & day & $\mathrm{S}$ & $1 / 86400$ & $\mathrm{~T}$ \\
\hline debyes & Debyes & N/A & N/A & $\left(\mathrm{M} / \mathrm{L} \mathrm{T}^{2}\right)^{1 / 2}$ \\
\hline $\operatorname{deg}$ & degrees & $\mathrm{rad}$ & 0.017453 & N/A \\
\hline degree & degrees & $\mathrm{rad}$ & 0.017453 & N/A \\
\hline dyn & dynes & $\mathrm{N}$ & $1 . \times 10^{-5}$ & $\mathrm{M} \mathrm{L} / \mathrm{T}^{2}$ \\
\hline dynes & dynes & $\mathrm{N}$ & $1 . \times 10^{-5}$ & $\mathrm{M} \mathrm{L} / \mathrm{T}^{2}$ \\
\hline $\mathrm{f}$ & Fahrenheit & $\mathrm{C}$ & $(f-32) \times(5 / 9)$ & $\mathrm{K}$ \\
\hline $\mathrm{ft}$ & foot & $\mathrm{m}$ & 0.3408 & $\mathrm{~L}$ \\
\hline furlong & furlong & $\mathrm{m}$ & $2.01168 \times 10^{2}$ & $\mathrm{~L}$ \\
\hline $\mathrm{ft}$ & foot & $\mathrm{m}$ & 0.3408 & $\mathrm{~L}$ \\
\hline furlong & furlong & $\mathrm{m}$ & $2.01168 \times 10^{2}$ & $\mathrm{~L}$ \\
\hline $\mathrm{g}$ & gm & $\mathrm{kg}$ & $1 . \times 10^{-3}$ & $\mathrm{M}$ \\
\hline gal & gallon & $\mathrm{m}^{3}$ & $3.7854 \times 10^{-3}$ & $\mathrm{M}^{3}$ \\
\hline gas & gas phase & $\mathrm{N} / \mathrm{A}$ & $\mathrm{N} / \mathrm{A}$ & $\mathrm{N} / \mathrm{A}$ \\
\hline $\mathrm{gm}$ & $\mathrm{gm}$ & $\mathrm{kg}$ & $1 . \times 10^{-3}$ & $\mathrm{M}$ \\
\hline gram & $\mathrm{kg}$ & $\mathrm{kg}$ & $1 . \times 10^{-3}$ & $\mathrm{M}$ \\
\hline hc & hydraulic cond. & $\mathrm{N} / \mathrm{A}$ & $1.03910 \times 10^{-7}$ & $\mathrm{~N} / \mathrm{A}$ \\
\hline
\end{tabular}


Table 4.3. Recognized Units (contd)

\begin{tabular}{|c|c|c|c|c|}
\hline Notation & Description & SI Equivalent & Conversion to SI & Base Units \\
\hline hour & hour & $\mathrm{s}$ & $1 / 3600$ & $\mathrm{~T}$ \\
\hline $\mathrm{hp}$ & horsepower & $\mathrm{W}$ & 745.7 & $\mathrm{M} \mathrm{L}^{2} / \mathrm{T}^{3}$ \\
\hline $\mathrm{hr}$ & hour & $\mathrm{s}$ & $1 / 3600$ & $\mathrm{~T}$ \\
\hline in & inch & $\mathrm{m}$ & $2.54 \times 10^{-2}$ & $\mathrm{~L}$ \\
\hline $\mathrm{j}$ & Joule & $\mathrm{J}$ & 1. & $\mathrm{M} \mathrm{L}^{2} / \mathrm{T}^{2}$ \\
\hline $\mathrm{k}$ & Kelvin & $\mathrm{C}$ & 1. & $\mathrm{~K}$ \\
\hline $\mathrm{kg}$ & kilogram & $\mathrm{kg}$ & 1. & $\mathrm{M}$ \\
\hline kgmol & kilogram-mole & kgmol & 1. & Mo \\
\hline 1 & liter & $\mathrm{m}^{3}$ & $1 . \times 10^{-3}$ & $\mathrm{~L}^{3}$ \\
\hline $1 \mathrm{~b}$ & pound & $\mathrm{kg}$ & 0.45359 & $\mathrm{M}$ \\
\hline lbm & pound & $\mathrm{kg}$ & 0.45359 & M \\
\hline lbmol & pound-mol & kgmol & 0.45359 & Mo \\
\hline liq & liquid & N/A & N/A & N/A \\
\hline liter & liter & $\mathrm{m}^{3}$ & $1 . \times 10^{-3}$ & $\mathrm{~L}^{3}$ \\
\hline $\mathrm{m}$ & meter & $\mathrm{m}$ & 1. & $\mathrm{~L}$ \\
\hline $\mathrm{mg}$ & milligram & $\mathrm{kg}$ & $1 . \times 10^{-6}$ & $\mathrm{M}$ \\
\hline $\min$ & minute & $\mathrm{s}$ & $1 / 60$ & $\mathrm{~T}$ \\
\hline $\mathrm{ml}$ & milliliter & $\mathrm{m}^{3}$ & $1 . \times 10^{-6}$ & $\mathrm{~L}^{3}$ \\
\hline $\mathrm{mm}$ & millimeter & $\mathrm{m}$ & $1 . \times 10^{-3}$ & $\mathrm{~L}$ \\
\hline $\mathrm{mol}$ & mole & kgmol & $1 . \times 10^{-3}$ & Mo \\
\hline mole & mole & kgmol & $1 . \times 10^{-3}$ & Mo \\
\hline $\mathrm{p}$ & Poise & $\mathrm{Pa} \mathrm{s}$ & $1 . \times 10^{-1}$ & $\mathrm{M} / \mathrm{L} \mathrm{T}$ \\
\hline $\mathrm{pa}$ & Pascal & $\mathrm{Pa}$ & 1. & $\mathrm{M} / \mathrm{L} \mathrm{T}^{2}$ \\
\hline pci & pico Curies & N/A & N/A & $\mathrm{N} / \mathrm{A}$ \\
\hline psi & $1 \mathrm{bs} / \mathrm{in}^{2}$ & $\mathrm{~Pa}$ & 6894.8 & $\mathrm{M} / \mathrm{L} \mathrm{T}^{2}$ \\
\hline $\mathrm{r}$ & Rakine & $\mathrm{K}$ & & $\mathrm{K}$ \\
\hline $\mathrm{rad}$ & radians & $\mathrm{rad}$ & 1. & $\mathrm{~N} / \mathrm{A}$ \\
\hline rod & rod & $\mathrm{m}$ & 5.0292 & $\mathrm{~L}$ \\
\hline $\mathrm{s}$ & second & $\mathrm{s}$ & 1. & $\mathrm{~T}$ \\
\hline sec & second & $\mathrm{s}$ & 1. & $\mathrm{~T}$ \\
\hline slug & slug & $\mathrm{kg}$ & 14.594 & $\mathrm{M}$ \\
\hline sol & solid & $\mathrm{N} / \mathrm{A}$ & $\mathrm{N} / \mathrm{A}$ & $\mathrm{N} / \mathrm{A}$ \\
\hline solid & solid & $\mathrm{N} / \mathrm{A}$ & N/A & N/A \\
\hline $\mathrm{w}$ & Watt & $\mathrm{W}$ & 1. & $\mathrm{M} \mathrm{L}^{2} / \mathrm{T}^{3}$ \\
\hline water & water & N/A & N/A & N/A \\
\hline week & week & $\mathrm{s}$ & $1 / 604800$ & $\mathrm{~T}$ \\
\hline wh & water head & $\mathrm{Pa}$ & $9.79353 \times 10^{3}$ & $\mathrm{M} / \mathrm{L} \mathrm{T}^{2}$ \\
\hline wk & week & $\mathrm{s}$ & $1 / 604800$ & $\mathrm{~T}$ \\
\hline yd & yard & $\mathrm{m}$ & 0.9144 & $\mathrm{~L}$ \\
\hline year & year & $\mathrm{S}$ & $1 / 31557600$ & $\mathrm{~T}$ \\
\hline $\mathrm{yr}$ & year & $\mathrm{s}$ & $1 / 31557600$ & $\mathrm{~T}$ \\
\hline
\end{tabular}


The units listed with no SI equivalent are primarily descriptive units that may be included in a unit character string to increase its readability. The $h c$ unit, however, is uniquely reserved for specifying rock/soil hydraulic conductivity that is normally expressed in velocity units. Without the $h c$ unit, the input for rock/soil permeability is interpreted as an intrinsic permeability value. The STORM simulator does not apply hydraulic conductivity values directly, but instead first converts hydraulic conductivity values into intrinsic permeability values using the density and viscosity of water at $20^{\circ} \mathrm{C}$. Therefore, when rock/soil permeability values are read as hydraulic conductivity, the associated unit character string should include the $h c$ unit (for example, hc $\mathrm{cm} / \mathrm{hr}$ ).

As an example, the standard units for thermal conductivity are $\mathrm{W} / \mathrm{K} \mathrm{m}$; however, a user may prefer to specify thermal conductivity in the standard English units of Btu in $/ \mathrm{hr} \mathrm{ft}^{2} \mathrm{~F}$. In this case, the user enters the character string Btu in $/ \mathrm{hr} \mathrm{ft}^{2} \mathrm{~F}$ for the unit data item. Note the carat $\left(^{\wedge}\right)$ symbol is used to indicate an exponential. During simulator execution, the string of English units for thermal conductivity is translated into the primary unit form, $\mathrm{m} / \mathrm{s}^{3} \mathrm{~kg} \mathrm{~K}$, and compared against the standard unit form. If the primary unit form does not agree with the standard unit form, an error message is generated by the STORM simulator and the program execution stops. Otherwise, if the comparison is successful, the input data for thermal conductivity, that precedes its unit string, is converted to SI units.

\subsection{Card Descriptions}

Formatting instructions for the input cards are provided in Appendix A. This section provides a brief synopsis of each input card with emphasis on its purpose and application. Italicized words refer to specific files, cards, options, and data entries shown in the card formats in Appendix A.

\subsubsection{Simulation Title Card}

This card primarily provides a means to document a simulation. Information recorded in this card is rewritten to the output file that then serves as a permanent record of the simulation. The user is encouraged to use descriptive titles and to briefly record the specifics and purpose of the simulation in the Simulation Notes section of the card. This record becomes especially valuable, when the user is making repeated simulations with small modifications to the input parameters. The changes required in either the Simulation Title or Simulation Notes will be invaluable when reviewing archived output files.

\subsubsection{Solution Control Card}

This card controls many general operational aspects of a simulation. Simulations are executed in one of three Execution Modes: Normal, Restart, or Initial Conditions. In the Normal mode, the simulation executes from initial conditions specified with the Initial Conditions Card, and no restart file is required. The Restart mode requires a restart file generated from a previous execution. Unless specified with an Overwrite indicator, initial conditions specified on the Initial Conditions Card are ignored. Restart files are generated during each plot file write sequence, and have name extensions that correspond to the generating time step (for example, the file restart.28 is generated after time step 28). Restart files contain principal field variables and time information. The Initial Conditions mode only executes through the initial checks on the input; no time steps are executed. The No Flow option (Water/Transport mode only) is used for transporting solutes through a steady-state flow field. For these simulations, the flow field is computed once during the initial time step and then remains unaltered. The Dynamic Domain option 
(Water mode only) conserves computations by temporarily removing nodes from the computational domain where changes in the flow field are insignificant. The active computational domain is updated every time step.

The Operation Mode option determines the governing equations that are solved by the simulator. Because execution performance is indirectly proportional to the number of solved governing equations, the user selects an Operation Mode most appropriate for the problem of interest. For example, if the physical system to be modeled does not contain thermal or gas pressure gradients, the most appropriate operational mode is the Water operational mode, where only the water mass conservation equation is solved. Executing the described physical system under the Water-Air operational mode yields nearly identical results; however, the execution time is significantly increased. Considerable attention is given to the selection of an appropriate operational mode for the physical system of interest. In terms of efficient and representative simulations, it is equally important not to eliminate critical physical phenomena through an erroneous assumption, as it is not to solve superfluous governing equations. Each operational mode allows the solution of solute transport equations, indicated by including the keyword Transport in the operational mode (for example, Water-Air and Solute Transport). Solute transport is solved using the Patankar method, unless the keyword TVD appears, indicating the TVD method.

Execution periods refer to simulation time. The STORM simulator allows the user to specify single or multiple execution periods. For each execution period, the user can control the initial time step, maximum time step, time step acceleration factor, maximum number of Newton-Raphson iterations, and convergence criterion. Recommended value for the Time Step Acceleration Factor is 1.25, the Maximum Number of Newton-Raphson Iterations is 8, and the Convergence Criterion is 1.e-06. Simulations involving complex phase transitions often require more Newton-Raphson iterations to reach convergence because of the design of the phase transition algorithms. For these types of simulations a value of 16 is recommended for the Maximum Number of Newton-Raphson Iterations. Except under special circumstances, it is inadvisable to change the Convergence Criterion from its recommended value, which has proven through many applications to achieve a preferred balance between accuracy and execution speed.

Field variables, including physical, thermodynamic, and hydrologic properties, are defined in the finite-difference formulation at node centers. Conversely, flux variables are defined at node interfaces. Computation of flux variables requires knowledge of field variables at node interfaces. Such values are evaluated by averaging the field values for the two nodes adjoining an interfacial surface. Interfacial averaging schemes may be declared individually for each field variable through the Interfacial Averaging Variables input. The default interfacial averaging schemes for STORM are shown in Table 4.4.

For simulations of physical systems involving heat transfer, note that convergence problems may arise if the density properties are not averaged with upwind weighting. Likewise, infiltration problems typically demonstrate strong dependencies on the relative permeability of the infiltrating fluid.

\subsubsection{Grid Card}

The finite-difference formulation of the STORM simulator is based a Cartesian grid system. The Cartesian coordinate system is a "right-handed" system with the longitudinal axis (z-direction) aligned with the negative gravitational vector. For the Cartesian coordinate system, the terms West, South, and Bottom refer to the negative $\mathrm{x}-$, $\mathrm{y}$-, and $\mathrm{z}$-directions, respectively, and the terms East, North, and Top refer to the positive $\mathrm{x}-, \mathrm{y}-$, and $\mathrm{z}$-directions, respectively. Negative dimensional values are not recognized 
and axes are defined as positive toward increasing node numbers. The grid dimensions that are specified on the Grid Card refer to node surfaces; therefore, for grids with non-uniform spacing, one plus the number of nodes entries are required for each grid direction. Node volumes are defined by their bounding surfaces. Refer to the STOMP Theory Manual (White and Oostrom 1996) for a graphical description of the Cartesian coordinate systems.

Table 4.4. Default Interfacial Averaging Options

\begin{tabular}{||l|l||}
\hline \multicolumn{1}{|c|}{ Field Variable } & Interfacial Averaging \\
\hline Aqueous density & Upwind \\
\hline Aqueous relative permeability & Upwind \\
\hline Aqueous viscosity & Harmonic \\
\hline Dissolved air diffusion & Harmonic \\
\hline Gas density & Upwind \\
\hline Gas relative permeability & Upwind \\
\hline Gas viscosity & Harmonic \\
\hline Hydraulic dispersion & Harmonic \\
\hline Intrinsic permeability & Harmonic \\
\hline Solute diffusion & Harmonic \\
\hline Thermal conductivity & Harmonic \\
\hline Water vapor diffusion & Harmonic \\
\hline \hline
\end{tabular}

\subsubsection{Rock/Soil Zonation Card}

This card allows the user to partition the computational domain into rock/soil types. All active nodes within the computational domain must have an associated rock/soil type. Rock/soil types are defined with a unique name that must contain no more than 64 characters. Because most physical systems of interest contain formations or zones of rocks or soils, the computational domain also contains zones of nodes with similar hydrogeologic properties. With this card, the user defines the distribution of rock/soil zones. In subsequent input cards, hydrogeologic properties are defined with respect to the defined rock/soil types. This approach avoids having to specify hydrogeologic properties uniquely for every node. Generally, this approach is preferred. For simulations using statistically generated rock or soil formations, which require unique hydrogeologic properties for each node, no advantage is gained with this approach. The rock/soil types are extended to the boundary surfaces for nodes adjacent to boundary surfaces. Therefore, field variables for boundary surfaces are computed using the rock/soil type property descriptions from the adjacent node. Hydrogeologic properties for rock/soil types are specified through the Mechanical Properties Card, Hydraulic Properties Card, Thermal Properties Card, Saturation Function Card, Aqueous Relative Permeability Function Card, Gas Relative Permeability Function Card, and Solute/Porous Media Interaction Card. A rock/soil type may be repeatedly applied to a node with the last definition being applied. For example, to simplify the zonation of a problem with a dominant rock/soil type and isolated pockets or bands of another rock/soil type, the user initially declares the dominant rock/soil type as covering the entire problem domain. With subsequent lines, the user can 
overwrite the original rock/soil type for selected nodes with another rock/soil type. This layering approach is recommended to avoid nodes with undeclared rock/soil types.

\subsubsection{Mechanical Properties Card}

This card allows the user to assign values to the particle density, porosity, specific storativity, compressibility, and tortuosity function for each defined rock/soil type. Every rock/soil type defined on the Rock/Soil Zonation Card must be referenced. Particle Density represents the rock grain density. This value can be defaulted to $2650 \mathrm{~kg} / \mathrm{m}^{3}$ by using a null entry for both the particle density and its associated unit. Total Porosity refers to total connected and unconnected pore volumes. Diffusive Porosity refers only to the connected pore volume. As with Particle Density, the Specific Storativity can be defaulted by using a null entry for both the Specific Storativity and its associated Units. Default specific storativity is computed from the Diffusive Porosity and with a default value of $1 . \times 10^{-7} 1 / \mathrm{Pa}$ for the compressibility. Tortuosity functions are required for simulations that involve solute transport or diffusion of components through phases (for example, water vapor diffusing through the gas phase). Tortuosities can be computed as either constants that require input values, or as functions of the phase saturation and diffusive porosity through the Millington and Quirk function. Refer to the STOMP Theory Manual (White and Oostrom 1996) for a description of the Millington and Quirk tortuosity function.

\subsubsection{Hydraulic Properties Card}

This card allows the user to assign values to the intrinsic permeability for each defined rock/soil type. Every rock/soil type defined on the Rock/Soil Zonation Card must be referenced. Intrinsic Permeability can be declared directly or through entering the Hydraulic Conductivity at reference conditions, where reference conditions refer to atmospheric pressure and $20^{\circ} \mathrm{C}$. By default, the STORM simulator reads the permeability values on this card as intrinsic permeabilities, unless the character string $h c$ is included in the associated units. Default units of $\mathrm{m}^{2}$ are applied to null entries for the units associated with permeability values. With the STORM simulator, a primary assumption is that principal components of the intrinsic permeability tensor are aligned with the principal coordinate directions. The STOMP Theory Manual (White and Oostrom 1996) has a description of the conversion of hydraulic conductivity to intrinsic permeability at reference conditions.

\subsubsection{Thermal Properties Card}

This card allows the user to assign values to the thermal conductivity and specific heat for each defined rock/soil type. Every rock/soil type defined on the Rock/Soil Zonation Card must be referenced. This card is required only for simulations involving the solution of the energy conservation equation. Declaration of the thermal conductivity depends on the operational mode and function option. (The STOMP Theory Manual (White and Oostrom 1996) describes thermal conductivity functions.) The Constant option fixes thermal conductivity to a constant value independent of temperature or saturation. The Parallel option requires the thermal conductivity of the soil grains and models thermal conductivity with an equivalent parallel path model dependent on porosity, phase saturations, and temperature. The Linear and Somerton options scale the thermal conductivity between the unsaturated and saturated values depending on phase saturation. A primary assumption with the STORM simulator is that principal components of the thermal conductivity tensor are aligned with the principal coordinate directions. 


\subsubsection{Saturation Function Card}

This card allows the user to declare and define a saturation-capillary pressure function for each defined rock/soil type. Every rock/soil type defined on the Rock/Soil Zonation Card must be referenced. Saturation function types and the required input items are primarily dependent on the operational mode. Although functional forms for the saturation-capillary pressure functions are preferred, tabular input is acceptable. By default, tabular data are interpolated linearly. Values beyond the table limits will be assigned either the table minimum or maximum values, as appropriate. For the van Genuchten function, the $m$ Parameter can be defaulted (with a null entry) or assigned a value. Default values will depend on which porosity distribution model (Mualem or Burdine) is chosen from the relative permeability function cards for the liquid phases. Hysteretic functions are those for which the drainage and imbibition scanning paths differ and that include capabilities for entrapment of fluids of lower wettabilities. Non-hysteretic functions require fewer input items than their hysteretic counterparts and use a single scanning path for drainage and imbibition events. Refer to the STOMP Theory Manual (White and Oostrom 1996) for a complete description of saturation-capillary pressure functions.

\subsubsection{Aqueous Relative Permeability Function Card}

This card allows the user to declare and define a relative permeability-saturation function for the aqueous phase for each defined rock/soil type. Every rock/soil type defined on the Rock/Soil Zonation card must be referenced. Aqueous relative permeability function types and the required input items are dependent on the operational mode. The Mualem and Burdine relative permeability functions are also dependent on the saturation function type and are strictly applicable to the van Genuchten and Brooks and Corey functions. For these functions, either the van Genuchten $m$ Parameter or the Brooks and Corey $\lambda$ Parameter can be defaulted to the values entered or defaulted with the saturation function. Although functional forms for the relative permeability-saturation functions are preferred, tabular input is acceptable. By default, tabular data is interpolated using linear interpolation. Values beyond the table limits are assigned appropriately either the table minimum or maximum values. (The STOMP Theory Manual (White and Oostrom 1996) has a complete description of aqueous relative permeability-saturation functions.)

\subsubsection{Gas Relative Permeability Function Card}

This card is used to declare and define a relative permeability-saturation function for the gas phase for each defined rock/soil type. Every rock/soil type defined on the Rock/Soil Zonation Card must be referenced. This card is required only for simulations involving flow or transport through the gas phase. Gas relative permeability function types and the required input items are dependent on the operational mode. The Mualem and Burdine relative permeability functions are also dependent on the saturation function type and are strictly applicable to the van Genuchten and Brooks and Corey functions. For these functions, either the van Genuchten $m$ Parameter or the Brooks and Corey $\lambda$ Parameter can be defaulted to the values entered or defaulted with the saturation function. Functional forms for the relative permeability-saturation functions are preferred; however, tabular input is acceptable. By default, tabular data are interpolated using linear interpolation; cubic spline interpolation is optional. Values beyond the table limits are assigned either the table minimum or maximum values, as appropriate. [White and Oostrom (1996) have a complete description of gas relative permeability-saturation functions.] 


\subsubsection{Solute/Fluid Interaction Card}

This card allows the user to define solutes, solute-fluid interactions, and solute radioactive decay paths. It is required only for simulations involving solute transport. The STORM simulator is capable of simulating any number of solutes with the assumption that solute concentrations remain dilute. (Solute concentrations do not vary the physical properties of the transporting fluid phases.) Solutes can decay radioactively to produce other solutes. For the loose coupling between parent solutes and progeny solutes to function properly, parent solutes must be defined on the card prior to definition of their progeny. The STORM simulator actually solves the transport equation for each solute sequentially and in reverse order from the definition list on this card. Solutes are defined by a unique solute name that must contain no more than 64 characters. Chain Decay Fraction relates a parent decay member with a progeny and refers to the fraction of the decaying parent that produces a particular progeny. Chain Decay Fraction inputs are fractional values from 0.0 through 1.0, inclusively. Solute-fluid interaction parameters such as molecular diffusion coefficients and interphase partition coefficients depend on the operational mode. Molecular diffusion coefficients refer to the diffusion of the solute through the transporting fluid phase outside of the porous media. Corrections for transport through porous media are handled within the STORM simulator. The interphase partition coefficients define the equilibrium distribution of solute between the active liquid phases. Partition coefficients that define the equilibrium adsorption of solute onto the solid phase are determined on the Solute/Porous Media Interaction Card. It is important for the user to carefully note the definitions and requested units for each interphase partition coefficient. The Aqueous-Gas Partition Coefficient is the ratio of the solute mass in the aqueous phase per unit mass of aqueous phase to the solute mass in the gas phase per unit volume of gas phase. [White and Oostrom (1996) have a more detailed description of the interphase partition coefficients, solute diffusion coefficients, radioactive decay rate equations, and radioactive chain decay fractions.]

\subsubsection{Solute/Porous Media Interaction Card}

This card allows the user to define solid-aqueous phase partition coefficients and porous media dependent hydraulic dispersivities. It is required only for simulations involving transport of solutes. This card differs from the Solute/Fluid Interaction Card because the input parameters declared are dependent on the solute and rock/soil type. For every solute defined on the Solute/Fluid Interaction Card, every rock/soil type defined on the Rock/Soil Zonation Card must be referenced. The Solid-Aqueous Partition Coefficient defines the interphase equilibrium of a solute adsorbed on the solid and dissolved in the aqueous phase. This definition refers to the concentration of solute adsorbed on the solid phase per unit mass of solid phase with the concentration of solute dissolved in the aqueous phase per unit mass aqueous phase. The longitudinal and transverse hydraulic dispersivities are properties only of the rock/soil type. Longitudinal Dispersivity is defined with respect to dispersion along the flow path and is assumed independent of the flow direction with respect to the porous media structure. Likewise, Transverse Dispersivity is defined with respect to dispersion transverse to the flow path, independent of the flow direction. [White and Oostrom (1996) present a more complete description of the solid-aqueous interphase partitioning and hydraulic dispersion of transported solutes.]

\subsubsection{Initial Conditions Card}

This card allows the user to assign starting values to primary and secondary hydraulic field variables by initializing primary variables. However, the current version of the STORM simulator does not allow 
the user to initialize flux or surface variables. Such initializations are useful for solving transport problems with steady flow fields. Transport problem simulations with STORM require the solution of the flow fields. The variables that may be initialized are dependent on the operational mode. For operational modes, default values for the initial conditions are specified within STORM as shown in Table 4.5.

Table 4.5. Initial Condition Default Values

\begin{tabular}{||l|c|c||}
\hline \multicolumn{1}{|c|}{ Initial Field Variable } & Symbol & Default Value \\
\hline Temperature $(\mathrm{C})$ & $\mathrm{T}$ & 20 \\
\hline Pressure of phase $\mathrm{j}(\mathrm{Pa})$ & $\mathrm{P}_{\mathrm{j}}$ & 101325 \\
\hline Saturation of phase $\mathrm{j}$ & $\mathrm{s}_{\mathrm{j}}$ & 0 \\
\hline Mole fraction of component $\mathrm{i}$ in phase $\mathrm{j}$ & $\mathrm{j}$ & 0 \\
\hline Solute concentration in phase $\mathrm{j}$ & $\mathrm{Sj}$ & 0 \\
\hline
\end{tabular}

For the Water, Water-Air, and Water-Air-Energy operational modes, STORM allows the user to specify the initial aqueous saturation by assigning initial conditions for any two of the following three variables: gas pressure, aqueous pressure, and aqueous saturation. The unassigned variable is computed from the other two through the declared saturation-capillary pressure functions. Initial conditions are declared repeatedly for a node with the application of last definition. Initial conditions for solutes are expressed in terms of solute per unit volume where the volume can refer to the total node volume (Volumetric), the aqueous-phase volume (Aqueous Volumetric), or the gas-phase volume (Gas Volumetric). Solute units are undefined and expressed as the user chooses (for example, Ci, pCi, gm, kg, mol, or kgmol). Although units for expressing solute quantity vary among solutes, units must be consistent for a single solute among all input data entries.

The gradient utilities of the initial condition card are invaluable and are used to advantage whenever possible. Gradients to the initial conditions allow the user to specify that initial value of a field variable varies along one or more directions in the physical domain. The initial condition variable assigned to an initial condition domain applies to the node with the lowest $x-, y-$, and $z$-direction indexes. If nonzero gradient values are specified, the initial condition values vary according to the gradients specified for each physical direction. Default values for the initial condition gradients are zero, indicating no variation. Gradient values are applicable only over the initial condition domain of the state. An exemplary application of the initial condition gradient utility occurs for problems that start with hydrostatic conditions. For these problems, the pressure at the lowest z-direction node positions is entered with a Z-Dir. Gradient that equaled minus the fluid density times the acceleration of gravity. For water at $20^{\circ} \mathrm{C}$, a z-direction gradient of $-9793.5192 \mathrm{~L} / \mathrm{m}$ yields constant head conditions that are equivalent to hydrostatic conditions. This approach is used to locate the position of a water table under no-recharge equilibrium conditions, given the rock/soil saturation function properties and a single pressure using an Initial Condition execution mode simulation.

\subsubsection{Boundary Conditions Card}

This optional card allows the user to control the simulation by defining time varying boundary conditions. Boundary conditions may be applied to any boundary surface or surface dividing active and inactive nodes. By default, all undeclared boundary surfaces have zero flux boundary conditions for flow and transport. Boundary conditions can be applied only to surfaces of active nodes. To apply a boundary 
condition to a boundary surface, the surface is referenced by the adjacent active node location and a direction with respect that node. To apply a boundary condition to a surface dividing an active and inactive node, the surface is referenced by the active node and the direction to the inactive node with respect to the active node. Boundary conditions vary within periods. The user is not allowed to assign multiple boundary conditions to a boundary surface during the same time period, but multiple boundary conditions can be applied over different time periods. The STORM simulator controls time steps to agree with time transitions in boundary conditions.

Application of boundary conditions requires an appropriate conceptualization of the physical problem and translation of that conceptualization into boundary condition form. The variety of boundary condition types available in the STORM simulator allows the user the flexibility to solve most subsurface flow and transport problems. In addition, the boundary condition card reader within the STORM simulator performs limited error checking on the boundary condition inputs. However, an error-free boundary condition card does not guarantee that the user did not create an ill-posed problem or an execution that successfully converges. For example, a mistake frequently made by STORM users is to specify infiltration rates at the top of a column with positive fluxes. Although this input is perfectly acceptable to the STORM boundary condition input reader, the specified condition actually withdraws flux from the top of the column because the z-axis and z-direction flux are positive in the upward direction.

The Boundary Surface Direction is specified with respect to the active node adjacent to a boundary surface. For the Cartesian coordinate system, the terms west, south, and bottom refer to the negative x-, $\mathrm{y}-$, and z-directions, respectively, and the terms east, north, and top refer to the positive $\mathrm{x}-$, $\mathrm{y}^{-}$, and $\mathrm{z}-$ directions, respectively.

Boundary condition inputs depend on the operational mode, with the required inputs becoming more complex with increasing number of solved equations. Generally, the number of boundary types that must be declared for each boundary equals the number of solved governing flow and transport equations. The Dirichlet boundary type is used to specify a field value (for example, pressure, temperature, or solute concentration) at the boundary surface. The Neumann boundary type allows the user to specify a flux (for example, liquid phase flux, heat flux, or solute flux) at the boundary surface. The Zero Flux boundary type is used to impose no flow or transport conditions across the boundary. The Saturated boundary type is available only for 2-phase conditions and imposes total-liquid saturation conditions (for example, water table) at the boundary surface. The Unit Gradient boundary type imposes hydrostatic conditions across the boundary surface for the specified phase. The Free Gradient boundary type is available only for the Water operational mode and models a free drainage surface with respect to the aqueous phase. The Hydraulic Gradient boundary type is applied only to a column or plane of vertical surfaces. With this boundary type, the user specifies a fluid phase pressure at the lowest surfaces of a column or row. The simulator then computes fluid phase pressure for the remaining boundary surfaces assuming hydrostatic conditions for the fluid phase. The Initial Conditions boundary type fixes the boundary field variables (for example, pressure, temperature, or solute concentration) to the initial values of the field variables at the node adjacent to the boundary surface. This boundary type is invariant with time. Outflow boundary types are applicable only to solute and energy boundary conditions. This boundary type considers only advectively transported solute or energy to exit the computational domain. Diffusion transport across the boundary surface is neglected. The solute concentration boundary types (for example, Volumetric, 
Aqueous, or Gas Concentration) are equivalent to Dirichlet boundary types for solute transport. These boundary types differ by their definitions of solute concentration.

Time variations of the boundary conditions are controlled through declaring multiple boundary times. All Boundary Time inputs are referenced against the Initial Time specified on the Solution Control card or obtained from a restart file. A boundary condition declared with a single Boundary Time implies the boundary condition is time invariant; the specified Boundary Time represents the start time for the boundary condition. Prior to the start time, the boundary surface type is assumed to be Zero Flux. The specified boundary condition remains in effect from the start time until the execution completion. If a boundary condition is declared with multiple Boundary Times, the first time listed equals the start time, the last time listed equals the stop time, and the intermediate times are transition points. For simulation times outside of the start and stop time limits, Zero Flux boundary conditions apply. For simulation times between two Boundary Times, linear interpolation of the boundary conditions is applied. Step boundary condition changes are simulated by defining duplicate Boundary Times; the first time indicates the completion of the previous boundary condition and the second indicates the start of the new boundary condition. At the completion of the step boundary condition, another set of duplicate Boundary Time declarations is used. Step boundary conditions are convenient methods for introducing slugs of fluids, heat, or solute in conjunction with the Neumann boundary type.

\subsubsection{Source Card}

This card allows the user to control sources or sinks of mass, energy, and solutes by defining the timevarying sources. By definition, sinks are negative sources, and sources refer to an influx of mass, energy, or solute into a node. Sources can be specified for interior or boundary nodes and are functionally analogous to Neumann type boundary conditions. Sources applied to inactive nodes are not recognized. Sources are time varying; however, unlike boundary conditions, application of multiple sources to a node are allowed during the same time period. The STORM simulator controls time steps to agree with time transitions in sources. Source inputs depend on the operational mode, with the required inputs becoming more complex with an increasing number of solved equations. Sinks withdraw mass, energy, or solutes from a node. The physical properties for the fluids withdrawn through sinks equal those of the node. Sources inject mass, energy, or solutes into a node. The physical properties for fluids injected through sources are computed from the specified input parameters.

Sources of type Aqueous Volumetric and Aqueous Mass inject aqueous fluid. For nonisothermal problems, the injected fluid also transports an amount of heat into the node, based on the enthalpy of the entering aqueous fluid. Sources of types Gas Volumetric/Mass Fraction, Gas Volumetric/Relative Humidity, Gas Mass/Mass Fraction, and Gas Mass/Relative Humidity are similar to the two aqueous sources in that mass and heat injected into the node include contributions from water vapor and air. The source type suffix Mass Fraction indicates that water vapor and air concentrations in the gas phase are specified through inputs of mass fractions for these quantities. Similarly, the source type suffix Relative Humidity indicates that water vapor and air vapor concentrations in the gas phase are specified through inputs of relative humidities for the water. Energy sources are specified as either type Power or Power Density, where Power type sources inject energy per unit time and Power Density type sources inject energy per unit time per node volume. Solute sources inject solute mass in the assumed units for solute (for example, $\mathrm{Ci}$, pCi, gm, kg, mol, or kgmol ). 
Time variations of sources are controlled through declaring multiple source times. All Source Time inputs are referenced against the Initial Time specified on the Solution Control card or are obtained from a restart file. A source declared with a single Source Time implies the source is time invariant; the specified Source Time represents the start time for the source. Prior to the start time, the source is zero and from the start time to execution completion, the source is as specified. If a source is declared with multiple Source Times, the first time listed equals the start time, the last time listed equals the stop time, and the intermediate times are transition points. For simulation times outside of the start and stop time limits, zero source conditions apply. For simulation times between two Source Times, linear interpolation of the sources is applied. Step source changes are simulated by defining duplicate Source Times; the first time indicates the completion of the previous source and the second time indicates the start of the new source. At the completion of the step source another set of duplicate Source Time declarations is used. Step sources are convenient methods for introduction slugs of fluids, heat, or solute into an interior node.

\subsubsection{Output Control Card}

This card allows the user to control output written to the output file, plot file, and screen (that is, STDIO). The output file contains an interpreted and reformatted version of the input and simulation results for selected variables at selected reference nodes over the simulation period. The plot file contains values of geometric parameters and selected variables for the entire computational domain (both active and inactive nodes) at selected simulation times. A plot file always is generated at the conclusion of an execution. The output to the STDIO primarily comprises variable results of the reference node versus the simulation time or time step. The user is advised to request screen output, because well-chosen output may be invaluable in tracking the simulation progress and identifying possible input errors. If a suite of repetitive simulations is in progress, then screen output can be reduced to minimum values. As with other input cards, output options are dependent on the operational mode. With respect to this card, output options primarily refer to computed field and flux variables.

Reference node output is generated by selecting reference nodes and output variables. The user may request any number of reference nodes. However, reference node output was primarily designed for tracking the time evolution of selected variables at key nodes of interest. Reference nodes are defined with three indexes that indicate the $\mathrm{x}-, \mathrm{y}-$, and $\mathrm{z}$-direction coordinates of the node. Node numbering in the STORM simulator increments in the order $\mathrm{i}, \mathrm{j}$, and $\mathrm{k}$, where the indexes refer to the $\mathrm{x}-, \mathrm{y}-$, and z-directions, respectively, for Cartesian coordinate systems. Reference Node Screen Output Frequency and Reference Node Output File Frequency are parameters that indicate how often, with respect to time step, reference node output is written to STDIO and the output file. A frequency value of 1 indicates reference node output occurs every time step, whereas a frequency value of 10 indicates reference node output occurs every 10 time steps. The user controls the output time and length units and the number of significant digits reported to the various output media. Unless declared through the Output Time Units or Output Length Units input items, values for time and lengths recorded to the output media are expressed in units of seconds and meters, respectively. These inputs allow the user to customize time- and lengthscale units to those most appropriate for the solved problem. The Screen Significant Digits, Output File Significant Digits, and Plot File Significant Digits input items allow the user to customize the number of significant digits that appear in field and flux variable results written to the STDIO, output file, and plot file. Default value for the number of significant digits is 5 ; the minimum number of significant digits is 4 . Field and flux variables output for the reference nodes are selected from the list shown in Appendix A for each operational mode. The same list of variables for each operational mode is available for output to the 
plot files. Output units for all variables that have units can be specified immediately following the variable name. Variables without units require a null entry for the variable units. The null entries for variables with units yield default output units that are expressed in SI units.

Plot files are written by default at the conclusion of an execution and at each requested Plot File Output Time. Plot files contain geometrical data and selected field and flux variable results for every node in the computational domain. These files represent a snapshot of the simulation at a certain point in time. Requests for Plot File Output Times can be specified with user defined units. A restart file is generated with every plot file. Both restart and plot files are suffixed with a file name extension of a dot followed by an integer (for example, plot.567, restart.32). The extension integer corresponds with the time step for which the file was written. Both plot and restart files are written at the conclusion of a time step. Field and flux variables recorded to plot files are selected from the list shown for each operational mode in Appendix A (refer to the reference node variable options). Output units for all variables with units are specified in the input item immediately following the variable name. Variables without units require a null entry for the variable units. Null entries for variables with units yield default output units that are expressed in SI.

\subsubsection{Surface Flux Card}

This card allows the user to define surfaces to track fluxes of fluid mass, fluid volume, heat, or solutes. A surface defined with this card can be composed of rectangular areas of coplanar surfaces on exterior boundaries or between interior nodes. Output from the surface flux integration routines is written to the surface file and contain flux rate and integral data for each defined surface at every time step. The types of fluxes that can be tracked depend on the operational mode. Declaration of surfaces is similar to defining boundary condition surfaces. Surfaces are defined by referencing a group of coplanar nodes and a surface direction with respect to the nodes. For the Cartesian coordinate system, the terms West, South, and Bottom refer to the negative $x-, y-$, and z-directions, respectively; the terms East, North, and Top refer to the positive $\mathrm{x}-, \mathrm{y}-$, and z-directions, respectively. For example, a surface to track the flux rate and integral of a particular solute entering the water table is defined for a simulation with saturated conditions along the bottom boundary surface by referencing the node group along the bottom of the computational grid and defining the Surface Flux Orientation as Bottom. One surface flux rate and integral value is computed for each defined surface and represents the summation of surface flux contributions from the individual surfaces in the coplanar group of surfaces.

\subsubsection{Reactive Transport Control Card}

This card lists several parameters that control the execution of the reactive transport subroutines. The first line requires specification of the reactive transport Execution Mode. Currently, only one Execution Mode is available for the reactive transport subroutines: Normal. In the Normal mode, the simulation executes from initial conditions specified with the Reaction IC Card.

The second line requires the user to select the reactive transport Solution Mode. Two solution modes are available: Fully Coupled mode, in which the transport equations and the chemical reactions are solved simultaneously, and Operator Split mode, in which advective-diffusive transport equations and the chemical reactions are solved sequentially. 
The third line lists ten integer values that must be set to either zero or one. These integer values represent flags (or switches) for setting different options in STORM:

1. The "Aitken extrapolation scheme" switch accelerates the convergence of the Newton-Raphson iteration scheme related to the reactive transport solver.

2. The "Column scan" switch for 2-D problems enables the Gauss-Seidel iterative solver to scan in the horizontal, as well as the vertical direction when solving 2-D problems.

3. The "nonreacting solver" switch allows the user to solve problems with pure transport (no reactions) in the Fully Coupled solution mode.

4. The "effective reaction area" switch allows recalculation of the effective reaction surface area for each mineral at each node, as the minerals precipitate or dissolve.

5. The "texture solver coupled with transport solver" switch allows the recalculation of the mineral volume and radius as minerals precipitate and dissolve in slow reactions.

6. The "porosity updated with precipitation/dissolution" switch allows changes in porosity to be passed back to the water or gas flow subroutines.

7. The "lower boundary condition for all species" switch allows either a no flux or a specified concentration of zero at the lower boundary for all aqueous species.

8. The "water consumption in chemical reactions coupled with water flow" switch allows the net water consumption rate due to all chemical reactions to be coupled with the water flow subroutines.

9. The "gas consumption in chemical reactions coupled with water flow" switch allows the net gas consumption rate due to all chemical reactions to be coupled with the gas flow subroutines.

10. The "diffusive/dispersive time step limiting" switch allows time step size to be limited by the von Neumann condition from Equation (3.77).

The fourth line lists seven real values used to control the reactive transport solution scheme:

1. The "log maximum concentration" sets the maximum concentration allowed in the reaction point solver. A typical value is 1.0. Setting this value too high may prevent the point solver from converging, whereas setting the value too low may result in mass balance errors. If concentrations for a particular species are consistently reaching maximum, adding equilibrium or kinetic reactions that reduce the concentration may be appropriate.

2. The "time stepping parameter" is used to select the time-stepping scheme for reactive transport: a value of 0.0 gives an explicit scheme, 0.5 gives a Crank-Nicholson scheme, and 1.0 provides a fully implicit scheme, which is likely to be the most stable. The other six input variables allow the user to specify the maximum error allowed in solving various reactive transport subroutines.

3. The "error in nonreacting species" specifies the maximum error allowed in the solution of nonreacting species in subroutine y_nonreactc.

4. The "eror in equation point solver" specifies the maximum error allowed in the solution of the equation point solver in subroutine y_pteqsol. 
5. The "eror in transport point solver" specifies the maximum error allowed in the solution of the transport point solver in subroutine y_pttsolv3.

6. The "eror in texture solver" specifies the maximum error allowed in the solution of the texture solver in subroutine d_texture.

7. The "eror in normal concentration solver" specifies the maximum error allowed in the solution of normal concentrations.

\subsubsection{Aqueous Species Card}

This card lists the aqueous species to be considered in the simulation. Examples of aqueous species include ions such as $\mathrm{HCO}_{3}^{-}$and dissolved gases such as $\mathrm{O}_{2}(\mathrm{aq})$. Required input includes the overall charge of the aqueous species and the hard-core diameter. Values of these input parameters for many aqueous species are found in the EQ3/6 database file data0.com.R2, for example. Also required is a list of the elements contained in each species. All aqueous species listed must correspond in number and order to the solutes listed in the Solute/Fluid Interaction card.

\subsubsection{Gas Species Card}

This card lists gas phase species to be considered in the simulation. Examples of gas phase species include $\mathrm{CO}_{2}(\mathrm{~g})$ and $\mathrm{O}_{2}(\mathrm{~g})$. These species must be related by Henry's Law Constant to an aqueous species specified in the Aqueous Species card. Required input includes the diffusion coefficient of the gas phase species and the Henry's Law equilibrium coefficient. The Henry's Law equilibrium coefficient may be specified as a constant or as a polynomial function of temperature with a user-specified number of terms. Additionally, a list of the elements contained in each gaseous species is required.

\subsubsection{Components Card}

The Components card provides a way to track the total mass of a given element in solution. For example, the user wishes to track the total molar concentration of aluminum in solution in a simulation involving $\mathrm{Al}(\mathrm{OH})_{3}(\mathrm{aq})$ and $\mathrm{Al}(\mathrm{OH})_{4}{ }^{-}$. Each mole of $\mathrm{Al}(\mathrm{OH})_{3}(\mathrm{aq})$ contains one mole of $\mathrm{Al}$, and each mole of $\mathrm{Al}(\mathrm{OH})_{4}{ }^{-}$also contains one mole of $\mathrm{Al}$. Therefore, the total molar concentration of $\mathrm{Al}$ in solution would be equal to the molar concentration of $\mathrm{Al}(\mathrm{OH})_{3}(\mathrm{aq})$ plus the molar concentration of $\mathrm{Al}(\mathrm{OH})_{4}{ }^{-}$. The title given to the total concentration in the components card is user-defined; in this example, a likely choice would be "Al(total)." The stoichiometric coefficients for each species, $\mathrm{Al}(\mathrm{OH})_{3}(\mathrm{aq})$ and $\mathrm{Al}(\mathrm{OH})_{4}{ }^{-}$, would each be specified as 1.0. Aqueous species listed must be defined previously in the Aqueous Species Card.

\subsubsection{Solid Species Card}

This card lists and describes all solid species in the simulation. Examples of solid species include silicate or carbonate minerals, metals, or soil organic matter. Solid species are defined by specifying the mass density of the solid species and the mole fraction (stoichiometric coefficient) and name of each element in the solid species. A list of element molecular weights is stored internally in STORM. 


\subsubsection{Fast Reactions Card}

This card lists and describes all aqueous fast (equilibrium) reactions considered in the simulation. Aqueous species used in each reaction must be defined previously in the Aqueous Species Card. Fast reactions are defined by specifying the number of moles of each aqueous species participating in the reaction. The equilibrium coefficient must also be specified, either as a constant or as a polynomial function of temperature with a user-specified number of terms.

\subsubsection{Kinetic Aqueous Reactions Card}

This card lists and describes all kinetic reactions involving only aqueous species. Aqueous species used in each reaction must be defined previously in the Aqueous Species card. An example of kinetic aqueous reactions is the biodegradation of dissolved organic carbon. Kinetic aqueous reactions are defined by specifying the number of moles of each aqueous species participating in the reaction. The equilibrium coefficient must also be specified, either as a constant or as a polynomial function of temperature with a user-specified number of terms. A kinetic reaction rate coefficient must also be defined.

\subsubsection{Slow Reactions Card}

This card lists all kinetic reactions involving at least one solid species. Solid species in each reaction must have been defined in the Solid Species card. Each reaction may involve one or two solid species. There must be at least one slow reaction involving each solid species. Examples of slow reactions include mineral dissolution or precipitation, metal corrosion, or biodegradation of soil organic matter. Slow reactions are defined by specifying the number of moles of each aqueous and solid species participating in the reaction. The equilibrium coefficient must also be specified, either as a constant or as a polynomial function of temperature, with a user-specified number of terms. Various parameters related to the rate law must also be defined. Three different types of rate law may be specified. In the full mass action law, all aqueous species participating in the reaction are considered in the mineral saturation. In the reduced mass action law, only certain specified aqueous species affect the equilibrium of the reaction. The glass mass action law is similar to the reduced mass action law, with the additional convention that glass is only allowed to dissolve, not precipitate.

\subsubsection{Fluid Decay Card}

The option to simulate the radioactive decay of aqueous species is not currently implemented.

\subsubsection{Solid Decay Card}

The option to consider the radioactive decay of solid species is not currently implemented.

\subsubsection{Lithology Card}

This card specifies the relative volume fraction and specific surface area, as defined in Equation (2.23) , for each solid species defined in the Solid Species card. These values must be separately defined for each of the porous materials specified in the Rock/Soil Zonation Card described in Section 4.4.4, or the 
solution will fail at the first time step. The porous materials should have the same names and be listed in the same order as in the Rock/Soil Zonation Card.

\subsubsection{Reaction BC Card}

This card lists the Dirichlet boundary conditions specified at the upper boundary. The aqueous solution may be specified to be in equilibrium with any solid phase (slow) reactions or kinetic aqueous reactions. Also, the concentration of any aqueous and gas species may be specified. If, for instance, only the total concentration of an element is known, all species containing that element may be listed and the total concentration specified. The total concentration will be divided among the listed aqueous species according to equilibrium reactions given in the Fast Reactions card. For the Fully Coupled reactive transport solution scheme, these values override transport boundary conditions specified in the Boundary Conditions card. Aqueous species listed must be defined previously in the Aqueous Species card. Gas species listed must be defined previously in the Gas Species card.

\subsubsection{Reaction IC Card}

This card lists the initial conditions specified throughout the domain, excluding the upper boundary. The format is identical to the Reaction $B C$ card. If the first line of this input card is set equal to zero, the initial aqueous species concentrations are set equal to those specified at the upper boundary, and no further input is needed. If the first line of this input card is set equal to 1, the input format for this card is identical to that for the Reaction BC card. Initial concentrations may be specified for all aqueous and gas species. Aqueous species listed must be defined previously in the Aqueous Species card. Gas species listed must be defined previously in the Gas Species card. 


\subsection{Parameter Files}

This section describes the format and contents of the parameters file and the parameters.astct file, which are required to compile and execute the STORM simulator. Parameters are used by the FORTRAN programming language and compilers to allocate memory for storage of variables. The FORTRAN77 language is unable to allocate memory dynamically; therefore, all memory storage requirements must be defined at compilation time. No execution errors occur if the memory allocated is greater that required by the simulation, unless the memory requirements exceed the computer capabilities. The user avoids executing simulations that require virtual memory unless it is needed. The time required to swap data between the virtual memory storage device and the active memory typically yields poor execution speeds. The STORM simulator requires definitions of two types of parameters (declared and computed), prior to compilation. The user is responsible for properly assigning all of the declared parameters. Declared parameters are assigned by modifying the parameters file supplied with the STORM simulator using a text editor (word processor) or by creating a new parameters file. The equations for the computed parameters must be included in each parameters file following the declared parameters. The parameter definitions given in this manual represent minimum acceptable values. All declared parameters, except for switch type parameters, must have minimum values of one. Undersized parameters will generally yield execution errors that may or may not be detected by the system. Oversized parameters are permissible, but can result in excessive memory allocation. The parameters file defines memory limits associated with the flow portions of STORM, whereas the parameters.astct file defines memory limits associated with the reactive transport portions of STORM.

\subsection{Parameters File}

\subsubsection{Parallel Execution Parameters}

The LZPROC and LXPROC parameters specify the minimum number of processors needed in each grid direction. For instance, if the code is submitted to run on 256 processors, STORM will automatically divide the grid into $16 \times 16=256$ pieces, and the minimum number of processes in each direction should be, at most: PARAMETER(LZPROC $=16, \mathrm{LXPROC}=16)$.

\subsubsection{Solution/Simulation Parameters}

The "LNOTES" parameter equals the number of lines in the Simulation Notes field and should equal the variable Number of Simulation Note Lines on the Simulation Title card. The "LEPD" parameter equals the number of execution periods and should equal the variable Number of Execution Periods on the Solution Control card. A simulation with seven lines of simulation notes and four execution periods would have a solution/simulation parameter set that appears as PARAMETER(LNOTES=7, LEPD=4).

\subsubsection{Computational Domain Parameters}

The "LFX," "LFY," and "LFZ" parameters equal the number of nodes in the $\mathrm{x}, \mathrm{y}$, and $\mathrm{z}$ directions, respectively, for Cartesian coordinate systems. These parameters equal the variables X-Dir. Nodes, Y-Dir. Nodes, and Z-Dir. Nodes on the Grid card. The parameter "LAN" equals the number of active nodes and 
should equal the total number of nodes minus the number of inactive nodes. The parameter "LAD" equals the number of active dimensions, where active dimensions are coordinate directions with more than one node. The number of active dimensions is a number between one and three, inclusive. The parameter "LMNP" equals the minimum plane size and should equal the minimum of the following three products: "LFX*LFY," "LFY*LFZ," and "LFZ*LFX." A simulation with 2 active dimensions, having 22, 1, and 103 nodes in the $\mathrm{x}-, \mathrm{y}$-, and $\mathrm{z}$ - directions, respectively, and 51 inactive nodes has a computational domain parameter set that appears as

PARAMETER(LFX=22, LFY=1, LFZ=103)

PARAMETER $(\mathrm{LAN}=2215, \mathrm{LAD}=2, \mathrm{LMNP}=22)$

For a parallel simulation on 16 processors, the grid would automatically be divided into $4 \times 4=16$ pieces, so the dimensions in the $\mathrm{X}$ and $\mathrm{Z}$ directions could be reduced:

PARAMETER(LFX=22/LXPROC+3, LFY=1, LFZ=103/LZPROC+3)

\subsubsection{Operation Mode Switch Parameters}

The parameters "LT," "LL," "LG," and "LC" are switches for the energy, water mass, air mass, and solute conservation equations. For these switch-type parameters, a value of one indicates the equation is solved and a value of zero indicates the equation is unsolved. The switch combinations set with these parameters agrees with the operational mode. The parameters "LN", "LFC", "LS", "LPC" and "LALC" are for operational modes used in STOMP, but not currently implemented in STORM. These parameters should always be set to zero. A simulation using the Water-Air/Transport operational mode has an operational mode parameter set that appears as

$$
\begin{aligned}
& \text { PARAMETER }(\mathrm{LT}=0, \mathrm{LL}=1, \mathrm{LG}=1, \mathrm{LC}=1) \\
& \text { PARAMETER }(\mathrm{LN}=0, \mathrm{LFC}=0, \mathrm{LS}=0, \mathrm{LPC}=0, \mathrm{LALC}=0)
\end{aligned}
$$

\subsubsection{Linear System Solver Switch Parameters}

The parameters "LBD" and "LSP" are switches for the banded and SPLIB iterative solvers. For these switch type parameters, a value of one indicates the solver is active and value of zero indicates the solver is inactive. Only one solver can be active for a simulation, either the banded matrix or the SPLIB iterative solver. These parameters switches, similar to a toggle, are the only means the user has for declaring a linear equation solution scheme. A simulation that used the banded matrix linear system solver has a linear system solver parameter set that appears as PARAMETER(LBD=1, LSP=0).

\subsubsection{Boundary Condition Parameters}

The parameters "LBC" and "LBTM" equal the number of boundary surfaces and maximum number of boundary condition times. A Boundary Condition Domain as declared on the Boundary Conditions card may contain more than one boundary surface. The parameter "LBC" indicates the total number of boundary surfaces, not the number of boundary condition domains. Although they may refer to the same computational domain surface, boundary surfaces declared in one boundary condition domain differ from those declared in another. The maximum number of boundary condition times equals the maximum number of times declared for all boundary condition domains. The parameters "LFC" and "LFTM" equal 
the number of fixed condition volumes and the maximum number of fixed condition times. These relate to a feature of STOMP that has not been implemented in STORM. These two parameters may safely be set to 1 . A simulation with three boundary condition domains, the first with six boundary surfaces and 13 boundary condition times, the second with two boundary condition surfaces and 26 boundary condition times, and the third with 34 boundary condition surfaces and two boundary condition times has a boundary parameter set that appears as PARAMETER(LBC=42, $\mathrm{LBTM}=26, \mathrm{LFC}=1, \mathrm{LFTM}=1)$.

\subsubsection{Source Parameters}

The parameters "LSR" and "LSTM" equal the number of sources and maximum number of source times. Unlike the boundary condition parameters, a single source may contain more than one node, but is only counted as one with respect to the parameter "LSR." The maximum number of source times equals the maximum number of times declared for all sources. A simulation with three source domains, the first with six nodes and 13 source times, the second with two sources and 26 source times, and the third with 34 sources and two source times would have a source parameter set that appears as PARAMETER(LSR=3, LSTM=26).

\subsubsection{Rock Type and Solute Transport Parameters}

The parameters "LRC" and "LSOLU" equal the number of rock types and number of transported solutes, respectively. The number of rock types equals the number of rock/soil types listed on the Rock/Soil Zonation card. Because one rock/soil type can define the rock/soil type for more than one rock/soil zonation domain, the number of rock/soil types does not equal the Number of Rock/Soil Zonation Domains declared on the Rock/Soil Zonation card. Each rock/soil type has a unique name. The number of solutes should equal the number of solutes defined on the Solute/Fluid Interactions card. Each solute must have a unique name. A simulation with 14 rock/soil zonation domains, three rock/soil types, seven solutes for the Water-Air/Transport operational mode has a rock types and solutes parameter set that appears as PARAMETER(LRC=3, LSOLU=7).

\subsubsection{Output Parameters}

The parameters "LREF," "LPTM," and "LSF" equal the number of reference nodes, number of plot file output times, and the number of flux surfaces, respectively. The number of reference nodes equals the Number of Reference Nodes input on the Output Control card. The number of plot file output times equals the Number of Plot File Times input on the Output Control card. The plot file that is generated, by default at the conclusion of an execution is not counted as a plot file time. The number of flux surfaces should equal the Number of Surface Flux Inputs input on the Surface Flux card. A simulation with 4 reference nodes, 6 plot file output times (other than the plot file generated at the conclusion of the execution), and 23 flux surfaces would have an output parameter set that appears as

PARAMETER(LREF=4, LPTM=6, LSF=23).

\subsubsection{0 k-S-P Function Parameters}

The parameter "LTBL" equals the total number of table entries for relative permeability and saturation functions, respectively. The number of table entries equals the sum of Number of Table Entries on the 
Saturation Function, Aqueous Relative Permeability Function, and Gas Relative Permeability Function cards. A Water operational mode simulation with 2 rock/soil types, 34 tabular entries for the saturation function for the first rock/soil type, a declared non-hysteretic saturation function for the second rock/soil type, 27 tabular entries for the aqueous relative permeability for the first rock/soil type, and 31 tabular entries for the aqueous relative permeability for the second rock/soil type has a $k-S-P$ function parameter set that appears as PARAMETER(LTBL=92).

\subsubsection{Computed Parameters}

Computed parameters are arithmetic combinations of the declared parameters and must follow the declared parameters in the parameters file. Computed parameters related to the SPLIB library of linear system solvers are declared within the source code. Definitions of selected computed parameters relevant to STORM are given in Table 5.1. The computed parameter set for all simulations appears as

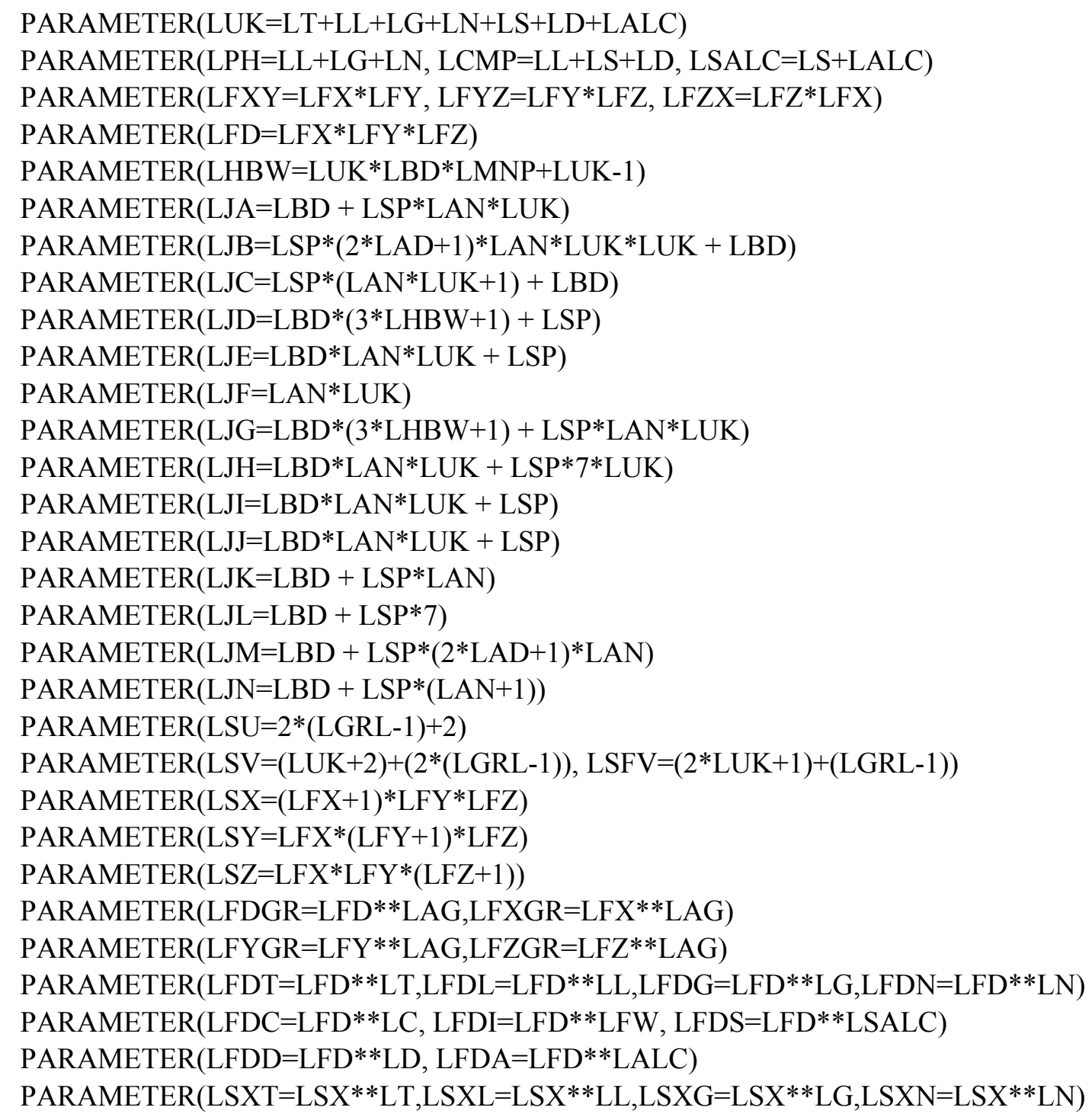




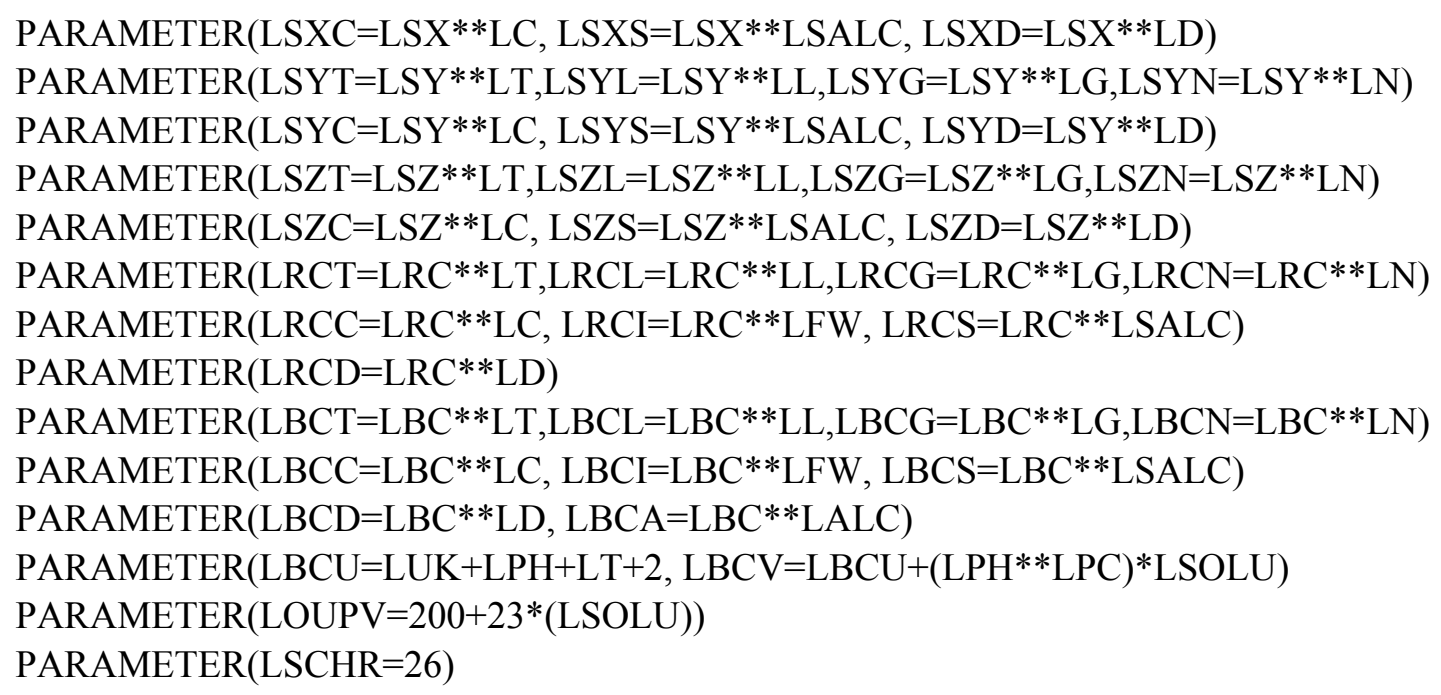

\section{2 parameters.astct File}

\subsubsection{Computational Domain Parameters}

The parameter maxxd specifies the maximum number of points in the vertical $(Z)$ direction, and is always set equal to the value of $L F Z+2$. The parameter maxyd specifies the maximum number of points in the horizontal $(\mathrm{X})$ direction, and is always set equal to the value of $\mathrm{LFX}+2$ :

parameter $(\operatorname{maxxd}=\mathrm{LFZ}+2, \operatorname{maxyd}=\mathrm{LFX}+2)$

\subsubsection{Species Parameters}

The parameter maxaqs specifies the maximum number of aqueous species. The parameter maxsld specifies the maximum number of solid species. The parameter maxgas specifies the maximum number of gaseous species. The parameter maxelem specifies the maximum number of elements in one species. The parameter maxaqs is always set equal to the value of LRC, which is defined in the parameters file. For example, a simulation with 20 solid species, 3 gas species, and 14 elements (maximum) per species would be parameterized using:

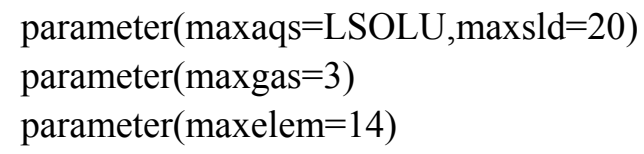


Table 5.1. Selected Computed Parameter Definitions

\begin{tabular}{|c|c|}
\hline Parameter & $\begin{array}{r} \\
\end{array}$ \\
\hline LUK & Number of solved coupled equations \\
\hline LPH & Number of phases \\
\hline LFXY & Number of nodes in the X-Y plane \\
\hline LFYZ & Number of nodes in the Y-Z plane \\
\hline LFZX & Number of nodes in the Z-X plane \\
\hline LFD & Number of nodes \\
\hline LHBW & Block banded matrix half-band width \\
\hline LJA-LJN & Linear system solution parameters \\
\hline LSV & Number of field variable indexes \\
\hline LSFV & Number of flux variable indexes \\
\hline LFD & Number of nodes \\
\hline LSX & Number of surfaces in the $\mathrm{x}$ - or $\mathrm{r}$-directions \\
\hline LSY & Number of surfaces in the y-direction \\
\hline LSZ & Number of surfaces in the z-direction \\
\hline LFDT & Number of energy field variables \\
\hline LFDL & Number of aqueous field variables \\
\hline LFDG & Number of gas field variables \\
\hline LFDC & Number of solute transport equation field variables \\
\hline LFDI & Number of solute transport equation field variables \\
\hline LSXT & Number of energy flux variables in the $\mathrm{x}$-direction \\
\hline LSXL & Number of aqueous flux variables in the $\mathrm{x}$-direction \\
\hline LSXG & Number of gas flux variables in the $\mathrm{x}$-direction \\
\hline LSXC & Number of solute flux variables in the $\mathrm{x}$-direction \\
\hline LSYT & Number of energy flux variables in the y-direction \\
\hline LSYL & Number of aqueous flux variables in the y-direction \\
\hline LSYG & Number of gas flux variables in the y-direction \\
\hline LSYC & Number of solute equation surfaces in the y-direction \\
\hline LSZT & Number of energy flux variables in the z-direction \\
\hline LSZL & Number of aqueous flux variables in the z-direction \\
\hline LSZG & Number of gas flux variables in the z-direction \\
\hline LSZC & Number of solute equation surfaces in the z-direction \\
\hline LRCT & Number of energy variable rock/soil types \\
\hline LRCL & Number of aqueous variable rock/soil types \\
\hline LRCG & Number of gas variable rock/soil types \\
\hline LRCC & Number of solute variable rock/soil types \\
\hline LBCT & Number of energy boundary condition variables \\
\hline LBCL & Number of aqueous boundary condition variables \\
\hline LBCG & Number of gas boundary condition variables \\
\hline LBCC & Number of solute boundary condition variables \\
\hline LBCU & Number of coupled boundary condition variables \\
\hline LBCV & Number of total boundary condition variables \\
\hline LOUPV & Number of output variables \\
\hline
\end{tabular}




\subsubsection{Reaction Parameters}

The parameter maxfrxn specifies the maximum number of fast reactions. The parameter maxsrxn specifies the maximum number of slow reactions. The parameter maxfdec specifies the maximum number of fluid decays. The parameter maxsdec specifies the maximum number of solid decays. The parameter maxkrxn specifies the maximum number of kinetic aqueous reactions. For example, a simulation with 20 fast reactions, 20 slow reactions, 1 fluid decay reaction, 1 solid decay reaction, and 1 kinetic aqueous reaction would be parameterized using:

$$
\begin{aligned}
& \operatorname{parameter}(\operatorname{maxfrxn}=20, \operatorname{maxsrxn}=20) \\
& \operatorname{parameter}(\operatorname{maxfdec}=1, \operatorname{maxsdec}=1) \\
& \text { parameter }(\operatorname{maxkrxn}=1)
\end{aligned}
$$

\subsubsection{Rock Type Parameters}

The parameter maxlith specifies the maximum number of lithologic units and is always set equal to the value of LRC, which is defined in the parameters file:

$$
\text { parameter(maxlith }=\mathrm{LRC})
$$

\subsubsection{Computed Parameters}

The parameter maxrxn specifies the maximum total number of reactions. The last line in the parameters.astct file is therefore

parameter $(\operatorname{maxrxn}=\max f r x n+\operatorname{maxkrxn}+\operatorname{maxsrxn}+\operatorname{maxfdec}+\operatorname{maxsdec})$ 


\subsection{Compilation and Execution}

The STORM simulator was designed primarily for execution on computers with UNIX ${ }^{(a)}$ operating systems. An assembled source code, however, can be compiled and executed on any computer with an ANSI FORTRAN compiler if the computer has sufficient memory. Although a general correspondence is present in computer design between memory size and execution speed, sufficient memory to compile and execute does not guarantee the machine has enough execution speed to complete a simulation within a reasonable amount of time. The STORM simulator should be thought of as a collection of source code libraries. The libraries that become part of the executable code depend on the operational mode, and each operational mode requires a particular group of library source codes. The normal procedure for building an executable version for a particular operational mode involves compiling the required group of library source codes, using the declared and computed parameters in the parameters and parameters.astct files to define memory requirements, and linking the compiled object files to create an executable. An alternative is to assemble or concatenate all the source code libraries required for a particular operational mode into a single file and subsequently compile the assembled source code. Either procedure can be performed manually or automatically. The make utility (Talbott 1988) that generates a sequence of commands for execution by the UNIX shell can be used to automatically compile and link the required source code libraries for a particular operational mode. A makefile, which contains a make utility instruction set, is designed to generate an executable or assembly source code for the STORM simulator.

\subsection{Compilation}

Executing the make command compiles and links all the source and object coding required for an operational mode into a single executable file. An example makefile is shown in Appendix B. Operational mode options are specified on the command line by the name of the executable. As an example, the following UNIX shell command string on a computer that supports the make utility generates an optimized executable of the Water-Air-Energy operational mode:

make storm3

where a file named "storm3" is generated. Table 6.1 shows the correspondence between the name of the executable and operational modes.

Table 6.1. Glossary of Operational Mode Indexes

\begin{tabular}{||c|l||}
\hline \hline Index & \multicolumn{1}{|c|}{ Operational Mode } \\
\hline storm1 & Water/Reactive Transport \\
\hline storm1m & Water/Reactive Transport-MPI Parallel \\
\hline storm2 & Water-Air/Reactive Transport \\
\hline storm3 & Water-Air-Energy/Reactive Transport \\
\hline
\end{tabular}

( ${ }^{a}$ UNIX is a registered trademark of AT\&T Information Systems. 
Assembly options are entered on a line within the file named Makefile, and assigned to a variable named FFLAGS. For example, the following line compiles the code on a SUN workstation with the options to generate a fast executable with the extended source code line length (132 characters) turned on.

$$
\text { FFLAGS }=\text {-fast }-\mathrm{e}
$$

The assembly process has certain requirements with respect to source files, the commons file, the parameters file, and the make description file. Storing all source files (those files that have $f$ or.$c$ extensions) and the commons file in a single directory is recommended. A path name to this directory can be defaulted into the make description file.

Executing make produces object files with .o extensions for every required FORTRAN source file. These object files appear in the current directory but are not necessary for code execution and can be discarded. Any modifications to the parameters file would require that the code be recompiled and linked. Executing make following a modification to the parameter file automatically regenerates the object and executable files. Because of the updating features in make, any recompilation using a different assembly option requires the user to remove all of the object files from the current directory.

\subsection{Execution}

Executing the STORM simulator is straightforward and only requires that an input file named input resides in the current directory. For simulations using prior STOMP simulations as initial conditions, a restart file named restart must also reside in the current directory. For simulations using prior STORM simulations as initial conditions, a restart file named restart and a backup file named backup must reside in the current directory. Restart files contain information on the flow variables and backup files contain information on the reactive transport variables. Because restart and backup files are created with an extension that corresponds with the generating time step, the user must rename the appropriate restart file to restart, and the appropriate backup file to backup. For a UNIX operating system, execution is started by typing in the path to and the name of the executable file. Execution is indicated by printing a STORM title banner and program disclaimer to the standard input/output device (for example, a monitor).

\subsubsection{Parallel Execution}

Parallel execution of STORM (on multiple processors) is slightly different from serial execution (on a single processor). Although the input files are identical, for parallel execution there must be one duplicate copy of the input file for each processor. If STORM is to be run on eight parallel processors, the eight input file copies must be named input_000, input_001, .. input_007. If needed, the restart file copies must be named restart_000, restart_001, ... input_007 and backup file copies must be named backup_000, backup_001, ... backup_007. An example script for running parallel STORM on eight

processors of the EMSL supercomputer mpp2 is shown in Appendix B. Copies of the input and restart file are made by the script.

\subsubsection{Error Messages}

Two types of error messages can be generated during a STORM execution. The first is a systemgenerated message that typically indicates a memory error, FORTRAN error, or other system error identified by the system. The second type of error messages generated by the STORM code typically 
refers to input, parameter, or convergence failure type messages. STORM-generated messages are divided into three categories according to severity. The most severe are the ERROR messages that abort the program execution. Undersized parameters are typical of errors that yield ERROR messages because execution of the simulator with undersized parameters may yield gross errors or, even worse, subtle errors that can pass undetected in the results. Next on the severity level are the WARNING messages that generally indicate to the user that a problem with the input file probably exists. The least severe are NOTE messages, which are used to record events such as the absence of an optional input card.

When an execution is terminated with a STORM-generated ERROR message, two types of information appear on the standard input/output device. The first piece of information is an error message with or without related data that indicates the nature of the error. To a limited degree, the STORM simulator also traps input formatting errors. Unfortunately, the resulting input error message that results may not always agree with the actual location of the identified input error. This situation arises because the original error may have skipped detection. For example, a missing input item on an input line is not identified until STORM identifies an incorrect data type or the end of the line was reached. The user will expect to find input errors slightly before the location indicated by the generating error message. The second type of information, which is generated with an ERROR message, is a calling sequence. The calling sequence is a slash-delineated list of routine names that were called prior to the identified error. The calling sequence for an input error on the Aqueous Relative Permeability card for the Water operational mode appears as

\section{/STORM1/RDINPT1/RDRPL/WRMSG}

where the called routine names are delineated with a forward slash. For this example, the main routine STORM1 called the subroutine RDINPT1 to read the input file for the Water operational mode. It, in turn, called RDRPL, the global routine, to read the Aqueous Relative Permeability card that identified an error and called WRMSG to write an error message to the standard input/output device.

Error messaging is more rigorous in the STOMP-derived flow and transport portions of the code. If an ambiguous run-time error occurs, it is likely related to the AREST-CT-derived reactive transport portions of the code. 


\subsection{Output Files}

The STORM simulator can generate, depending on the requested output and saturation function type, two data files for restarting a simulation and three simulation result files. Every execution produces an output file. If the simulation concludes without a fatal error, a restart and plot file are also generated. Restart and plot files names include an extension that corresponds to the time step at which the file was written. For example, a restart file named restart. 39 was written at the conclusion of the $39^{\text {th }}$ time step. A surface file is only generated when a Surface Flux card is included in an input file. All output files generated by the STORM simulator are ASCII text files.

\subsection{Output File}

An output file is generated with every execution. If a file named output already exists in the current directory, the file is erased and overwritten. The user is responsible for renaming previous output files prior to executing the STORM simulator. An output file begins with a welcome statement, a disclaimer, and a banner that appear similar to

Welcome to ...

STORM

Subsurface Transport Over Reactive Multiphases

This file was produced by STORM, a numerical simulator developed by the Pacific Northwest National Laboratory with support from the U.S. Department of Energy.

Results from this version of STORM should not be used for license related applications. Distribution of the STORM source code is strictly prohibited.

For inquiries or assistance: Call (509) 372-6132

\section{--- OUTPUT ---}

Following this banner is an input record that documents the simulation input. An input record is included on the output file to document the simulation in the event the input file is destroyed or becomes separated from the output file. The input record is formatted differently that an input file, but contains all of the information listed on an input file. Optional cards that are not included in the input file are noted in the input record. If an input error is identified by the STORM simulator, an error message appears in the input record at the point the error was noted. Input cards are read by the STORM simulator in a specific order and appear in that order in the input record. Because input cards can be organized randomly on the input file, the card order on the input record may not match that in the input file. 


\subsection{Refnod File}

The refnod (reference node) output file is a table of simulation data and selected reference node variables that are printed according the frequency requested on the Output Control card with the input item Reference Node Output File Frequency. After every 10 print records, a table column header is printed to delineate the columns. Each print record shows simulation data and reference node variable data for each reference node requested. Simulation data comprise the time step, the reference node number, the simulation time, the simulation time step, and the number of Newton-Raphson iterations required to reach convergence. Reference node variable data comprise values of the variables specified on the Output Control card through the Reference Node Variable inputs. Reference nodes are indexed by node number. The corresponding $\mathrm{x}-, \mathrm{y}-$, and $\mathrm{z}$-direction indexes are shown for each reference node on the line preceding the column headers; for example, a simulation with reference node 272 at $\mathrm{x}-, \mathrm{y}-$, and $\mathrm{z}-$ direction indexes of 2,3 , and 6 , respectively, has a reference node header line that appears as

$$
\text { Reference Node(s) ( 2, 3, 6: 272) }
$$

Column headers for reference node variables are delineated with an abbreviated title and associated units enclosed in brackets below the variable abbreviation. A portion of a reference node output record for a horizontal heat pipe problem, involving 50 nodes for time steps 70 through 73, appears as

\begin{tabular}{|c|c|c|c|c|c|c|c|}
\hline & & & & & & & \\
\hline Step & Node & Time & Timestep & Itr & $\mathrm{T}$ & SL & PL \\
\hline & & [day ] & [day ] & & & & {$[\mathrm{pa}$} \\
\hline 70 & 1 & $2.837 \mathrm{E}+01$ & $1.793 \mathrm{E}+00$ & 8 & $7.1893 \mathrm{E}+01$ & 9.9893E-01 & $1.0133 \mathrm{E}+05$ \\
\hline 70 & 50 & $2.837 \mathrm{E}+01$ & $1.793 \mathrm{E}+00$ & 8 & $1.3564 \mathrm{E}+02$ & 2.0992E-02 & $-1.9896 \mathrm{E}+08$ \\
\hline 71 & 1 & $3.061 \mathrm{E}+01$ & $2.242 \mathrm{E}+00$ & 9 & $7.1895 \mathrm{E}+01$ & 9.9903E-01 & $1.0133 \mathrm{E}+05$ \\
\hline 71 & 50 & $3.061 \mathrm{E}+01$ & $2.242 \mathrm{E}+00$ & 9 & $1.4039 \mathrm{E}+02$ & $1.9265 \mathrm{E}-02$ & $-2.2722 \mathrm{E}+08$ \\
\hline 72 & 1 & $3.341 \mathrm{E}+01$ & $2.802 \mathrm{E}+00$ & 9 & $7.1878 \mathrm{E}+01$ & 9.9933E-01 & $1.0133 \mathrm{E}+05$ \\
\hline 72 & 50 & $3.341 \mathrm{E}+01$ & $2.802 \mathrm{E}+00$ & 9 & $1.4598 \mathrm{E}+02$ & $1.7489 \mathrm{E}-02$ & $-2.6050 \mathrm{E}+08$ \\
\hline 73 & 1 & $3.692 \mathrm{E}+01$ & $3.503 \mathrm{E}+00$ & 8 & $7.1869 \mathrm{E}+01$ & 9.9951E-01 & $1.0133 \mathrm{E}+05$ \\
\hline 73 & 50 & $3.692 \mathrm{E}+01$ & $3.503 \mathrm{E}+00$ & 8 & $1.5225 \mathrm{E}+02$ & $1.5759 \mathrm{E}-02$ & $-2.9758 \mathrm{E}+08$ \\
\hline
\end{tabular}

The reference nodes are 1 and 50; the reference node variable data includes the temperature, reported in degrees Celsius, the aqueous saturation, and the aqueous pressure, reported in Pascal (absolute).

\subsection{Plot File}

A plot file is generated by default at the conclusion of every execution and, additionally when requested on the Output Control card with the Plot File Output Times input items. If a file named plot.xx (where $x x$ refers to the time step extension) already exists in the current directory, the file is erased and overwritten. The user is responsible for renaming previous plot.xx files before executing the STORM simulator. A plot file begins with a welcome statement, a disclaimer, and a banner that appear similar to 
Welcome to ...

\section{STORM}

Subsurface Transport Over Reactive Multiphases

This file was produced by STORM, a numerical simulator developed by the Pacific Northwest National Laboratory with support from the U.S. Department of Energy.

Results from this version of STORM should not be used for license related applications. Distribution of the STORM source code is strictly prohibited.

For inquiries or assistance: Call (509) 372-6132

--- PLOT ---

Following the header are data on the current time step, simulation time, and number of nodes in three coordinate directions. The remaining portion of a plot file comprises arrays of geometric and variable data arranged in groups with a title line indicating the type of data that follows. Data groups are arranged in rows of 10 data items. Each group contains a field or surface variable listed sequentially for every node or surface in the computational domain. The first four groups of data appear in every plot file and contain the $\mathrm{x}$ - or $\mathrm{r}$-direction positions, $\mathrm{y}$ - or $\theta$-direction positions, $\mathrm{z}$-direction positions, and volume for every node in the computational domain. The remaining data groups correspond to the requested Plot File Variables. All reactive transport output variables, such as aqueous species concentrations and solid species surface areas, are written by default. Field variable data groups list the value of the field variable at the node centroid. Surface variables or fluxes are written twice to the plot file. The first data group lists the value of the flux variable at the node centroid; the second lists the value at the node surfaces. Node centroid values for flux variables are simple arithmetic averages of the two node surface values.

Two post-processing programs are available, d_plot1d and d_plot2d. The program d_plot1d parses output data from a 1-D simulation into columnar format. The program d_plot2d parses data from a 2-D simulation into Spyglass Transform format.

\subsection{Surface File}

A surface file is generated during an execution if surfaces are defined on the Surface Flux card If a file named surface already exists in the current directory, the file is erased and overwritten. The user is responsible for renaming previous surface files prior to executing the STORM simulator. A surface file begins with a welcome statement, a disclaimer, and a banner that appear similar to

Welcome to ...

\section{STORM}

Subsurface Transport Over Reactive Multiphases

This file was produced by STORM, a numerical simulator

developed by the Pacific Northwest National Laboratory 
with support from the U.S. Department of Energy.

Results from this version of STORM should not be used

for license related applications. Distribution of the

STORM source code is strictly prohibited.

For inquiries or assistance: Call (509) 372-6132

\section{--- SURFACE ---}

After the header are surface flux rate and integral data. Surface flux information is written to the surface file at the end of every time step. The first column of data in the surface file is the time step in units specified with the Output Time Units variable on the Output Control card. Each surface flux output is written in two columns: the first is the surface flux rate at the current time step and the second is the integral of the surface flux rate from the beginning of the current simulation. Surface flux integral data are not carried over on restart simulations. Each column in the surface file is titled with an abbreviated header and associated units shown in brackets underneath. Definitions of abbreviations for the surface file are given in Table 7.1. Each header title is followed by an integer enclosed in parentheses that refers to the surface flux number defined on the Surface Flux card (i.e., the surface flux definition order).

\subsection{Restart File}

A restart file is generated by default at the conclusion of every execution and also when requested on the Output Control card with the Plot File Output Times input items. If a file named restart.xx (where $x x$ refers to the time step extension) already exists in the current directory, the file is erased and overwritten.

The user is responsible for renaming previous restart.xx files prior to executing the STORM simulator. A restart file begins with a welcome statement, a disclaimer, and a banner that appear similar to

Welcome to ...

STORM

Subsurface Transport Over Reactive Multiphases

This file was produced by STORM, a numerical simulator developed by the Pacific Northwest National Laboratory with support from the U.S. Department of Energy.

Results from this version of STORM should not be used for license related applications. Distribution of the STORM source code is strictly prohibited.

For inquiries or assistance: Call (509) 372-6132

--- RESTART --- 
Table 7.1. Surface Flux Column Title Abbreviations

\begin{tabular}{||l|l||}
\hline Abbreviation & \multicolumn{1}{|c||}{ Definition } \\
\hline UGM & Gas mass flux rate (x-direction) \\
\hline UGMS & Gas mass flux integral (x-direction) \\
\hline ULM & Aqueous mass flux rate (x-direction) \\
\hline ULMS & Aqueous mass flux integral (x-direction) \\
\hline UGV & Gas volumetric flux rate (x-direction) \\
\hline UGVS & Gas volumetric flux integral (x-direction) \\
\hline ULV & Aqueous volumetric flux rate (x-direction) \\
\hline ULVS & Aqueous volumetric flux integral (x-direction) \\
\hline UQV & Energy flux rate (x-direction) \\
\hline UQVS & Energy flux integral (x-direction) \\
\hline VGM & Gas mass flux rate (y-direction) \\
\hline VGMS & Gas mass flux integral (y-direction) \\
\hline VLM & Aqueous mass flux rate (y-direction) \\
\hline VLMS & Aqueous mass flux integral (y-direction) \\
\hline VGV & Gas volumetric flux rate (y-direction) \\
\hline VGVS & Gas volumetric flux integral (y-direction) \\
\hline VLV & Aqueous volumetric flux rate (y-direction) \\
\hline VLVS & Aqueous volumetric flux integral (y-direction) \\
\hline VQV & Energy flux rate (y-direction) \\
\hline VQVS & Energy flux integral (y-direction) \\
\hline WGV & Gas volumetric flux rate (z-direction) \\
\hline WGVS & Gas volumetric flux integral (z-direction) \\
\hline WLV & Aqueous volumetric flux rate (z-direction) \\
\hline WLVS & Aqueous volumetric flux integral (z-direction) \\
\hline WGM & Gas mass flux rate (z-direction) \\
\hline WGMS & Gas mass flux integral (z-direction) \\
\hline WLM & Aqueous mass flux rate (z-direction) \\
\hline WLMS & Aqueous mass flux integral (z-direction) \\
\hline WQV & Energy flux rate (z-direction) \\
\hline WQVS & Energy flux integral (z-direction) \\
\hline \hline
\end{tabular}

All data values in restart files are in SI units with pressures expressed in gauge. The first data line of a restart file contains the following timing and simulation information: time, time step, maximum time step, time step acceleration factor, convergence criterion, maximum number of Newton-Raphson iterations, number of time steps, and number of nodes. The remaining lines are node data lines that contain the following field variable data for the current time step for every node (one line per node): temperature, aqueous pressure, gas pressure, water vapor pressure, aqueous saturation, and gas saturation. If the simulation includes solute transport, the node data lines also contain solute volumetric concentrations for each solute. Moreover, if the simulation includes freezing water conditions, the node data lines also contain the frozen water pressure and frozen water saturation. All node data lines are 
terminated with an integer value for the phase condition. Refer to the STOMP Theory Manual (White and Oostrom 1996) for a discussion of phase conditions.

\subsection{Backup File}

A backup (reaction restart) file is generated by default at the conclusion of every execution and also when requested on the Output Control card with the Plot File Output Times input items. If a file named backup. $x x$ (where $x x$ refers to the time step extension) already exists in the current directory, the file is erased and overwritten. The user is responsible for renaming previous backup.xx files prior to executing the STORM simulator. The backup file contains additional field variable data not contained in the restart files, including aqueous species concentrations, mineral volume, radius and number of grains. These field variables are related to the AREST-CT-derived reactive transport portions of the code. 


\subsection{Model Verification}

In this section, several test problems are presented that compare STORM calculations to other solution methods to verify that the numerical algorithms are operating correctly. STORM is compared with analytical solutions for aqueous advection and diffusion, as well as gas phase advection and diffusion. Hand calculations are used to verify gas source/sink mass balance. EQ3/6 calculations are used to benchmark STORM simulations of quartz dissolution and calcite precipitation.

\subsection{Aqueous Phase Advection and Diffusion}

For the case of 1-D, steady-state diffusion and advection of a single aqueous species, with constant production from a single reaction with depth, Equations (2.17) through (2.27) simplify to

$$
W=-\left(\theta_{a} \mathbf{D}^{a}\right) \frac{\partial^{2} C^{a}}{\partial z^{2}}+\left(\theta_{a} \mathbf{V}_{a}\right) \frac{\partial C^{a}}{\partial z}
$$

Assuming production occurs over a finite depth $0<z<L$ with a specified concentration $\left(C_{0}^{a}\right)$ at the top boundary $(z=0)$ and no diffusive flux at the lower boundary $(z=L)$, the aqueous phase concentration of that species is given by

$$
C_{z}^{a}=\frac{\mathbf{D}\left(\mathrm{e}^{\frac{L \mathbf{v}}{\mathbf{D}}}-\mathrm{e}^{\frac{(L-z) \mathbf{v}}{\mathbf{D}}}\right) W+\mathbf{v}\left(C_{0}^{a} \mathbf{v}-W z\right)}{\mathbf{v}^{2}}
$$

where $\mathbf{V}=\theta_{a} \mathbf{V}_{a}$ and $\mathbf{D}=\theta_{a} \mathbf{D}^{a}$. A STORM simulation of Halite dissolution was run that corresponds to this analytical solution. Both the Fully Coupled and the Operator Split solution modes were used.

Output from STORM was compared with the analytical solution. The model parameters are listed in Table 8.1; the results are shown in Figure 8.1. Results for STORM run in the Fully Coupled solution mode and the analytical solution agree well. Results for STORM run in the Operator Split mode agree, if the time step is constrained to a maximum value of 0.5 hour.

Table 8.1. Simulation Parameters for Aqueous Phase Advection and Diffusion

\begin{tabular}{||l|l||}
\hline \multicolumn{1}{|c|}{ Parameter } & \multicolumn{1}{c||}{ Value } \\
\hline Temperature, $\mathrm{C}$ & 25 \\
\hline$C_{0}^{a}, \mathrm{~mol} \mathrm{~kg}^{-1}$ & $1.0 \times 10^{-7}$ \\
\hline$D^{a}, \mathrm{~m}^{2} \mathrm{~s}^{-1}$ & $5.0 \times 10^{-4}$ \\
\hline$V_{a}, \mathrm{~m} \mathrm{~s}^{-1}$ & $1.6 \times 10^{-8}$ \\
\hline$\theta_{\mathrm{a}}$ & $5.2 \times 10^{-2}$ \\
\hline$W, \mathrm{~mol} \mathrm{~m}^{-3} \mathrm{~s}^{-1}$ & $1.2 \times 10^{-9}$ \\
\hline
\end{tabular}




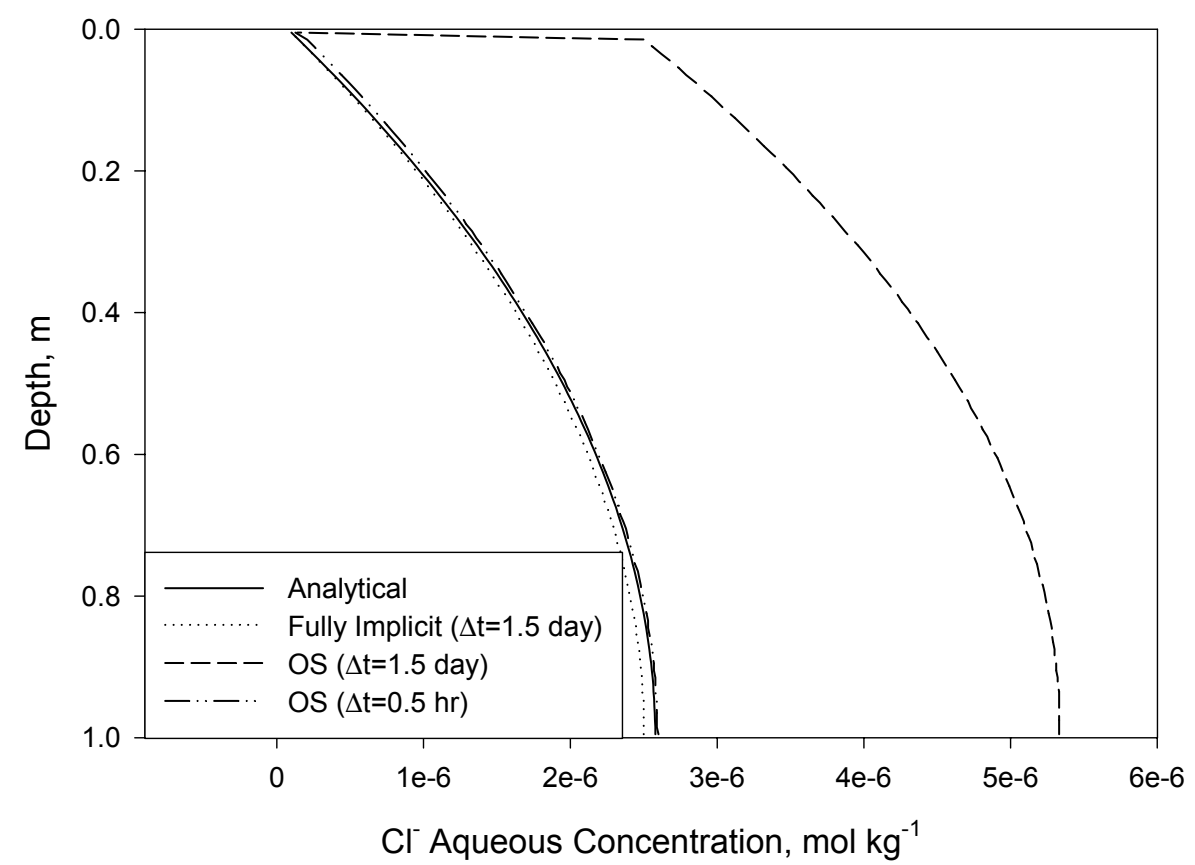

Figure 8.1. Comparison of STORM Results Using Either Fully Coupled or Operator Split Solution Modes to Analytical Solution for Constant Source, Advection, and Diffusion

\subsection{Gas Phase Advection and Diffusion}

\subsubsection{Gas Phase Diffusion Only}

For the case of 1-D, steady state diffusion of a single gas species, with constant production from a single reaction with depth, Equation (2.17) simplifies to

$$
W=-\left(\theta_{g} \mathbf{D}^{g} K_{H}^{\prime-1}+\theta_{a} \mathbf{D}^{a}\right) \frac{\partial^{2} C^{a}}{\partial z^{2}}
$$

Assuming production occurs over a finite depth $0<z<L$, with a specified concentration $\left(C_{0}^{a}\right)$ at the top boundary $(z=0)$ and no diffusive flux at the lower boundary $(z=L)$, the aqueous phase concentration of that gas species is given by

$$
C_{z}^{a}=C_{0}^{a}+\frac{W}{\left(\theta_{g} \mathbf{D}^{g} K_{H}^{\prime-1}+\theta_{a} \mathbf{D}^{a}\right)}\left(z L-\frac{z^{2}}{2}\right)
$$

and the gas phase concentration may be determined using Equation (2.16). A STORM simulation was run that corresponds to this analytical solution. The model parameters are listed in Table 8.2.

Results for simulations with two different $\mathrm{CO}_{2}(\mathrm{~g})$ production rates are shown in Figure 8.2 (open and closed diamond symbols). Results for STORM and the analytical solution agree quite well. 
Concentrations of $\mathrm{CO}_{2}(\mathrm{~g})$ shown are reasonable values found in vadose zones where organic matter is being decomposed by aerobic bacteria.

Table 8.2. Simulation Parameters for Gas Phase Diffusion

\begin{tabular}{|l|l|}
\hline \multicolumn{1}{|c|}{ Parameter } & \multicolumn{1}{c|}{ Value } \\
\hline Temperature, $\mathrm{C}$ & 15 \\
\hline$C_{0}^{a}$, atm & $3 \times 10^{-4}$ \\
\hline$K_{H}^{\prime}$ & 1.088 \\
\hline $\mathrm{D}^{\mathrm{g}}, \mathrm{m}^{2} \mathrm{~s}^{-1}$ & $1.6 \times 10^{-6}$ \\
\hline $\mathrm{D}^{\mathrm{a}}, \mathrm{m}^{2} \mathrm{~s}^{-1}$ & $5 \times 10^{-9}$ \\
\hline$\theta_{\mathrm{g}}$ & 0.24 \\
\hline$\theta_{\mathrm{a}}$ & 0.0266 \\
\hline $\mathrm{W}, \mathrm{mol} \mathrm{m}^{-3} \mathrm{~s}^{-1}$ & $4.65 \times 10^{-9}$ or $9.60 \times 10^{-9}$ \\
\hline \hline
\end{tabular}

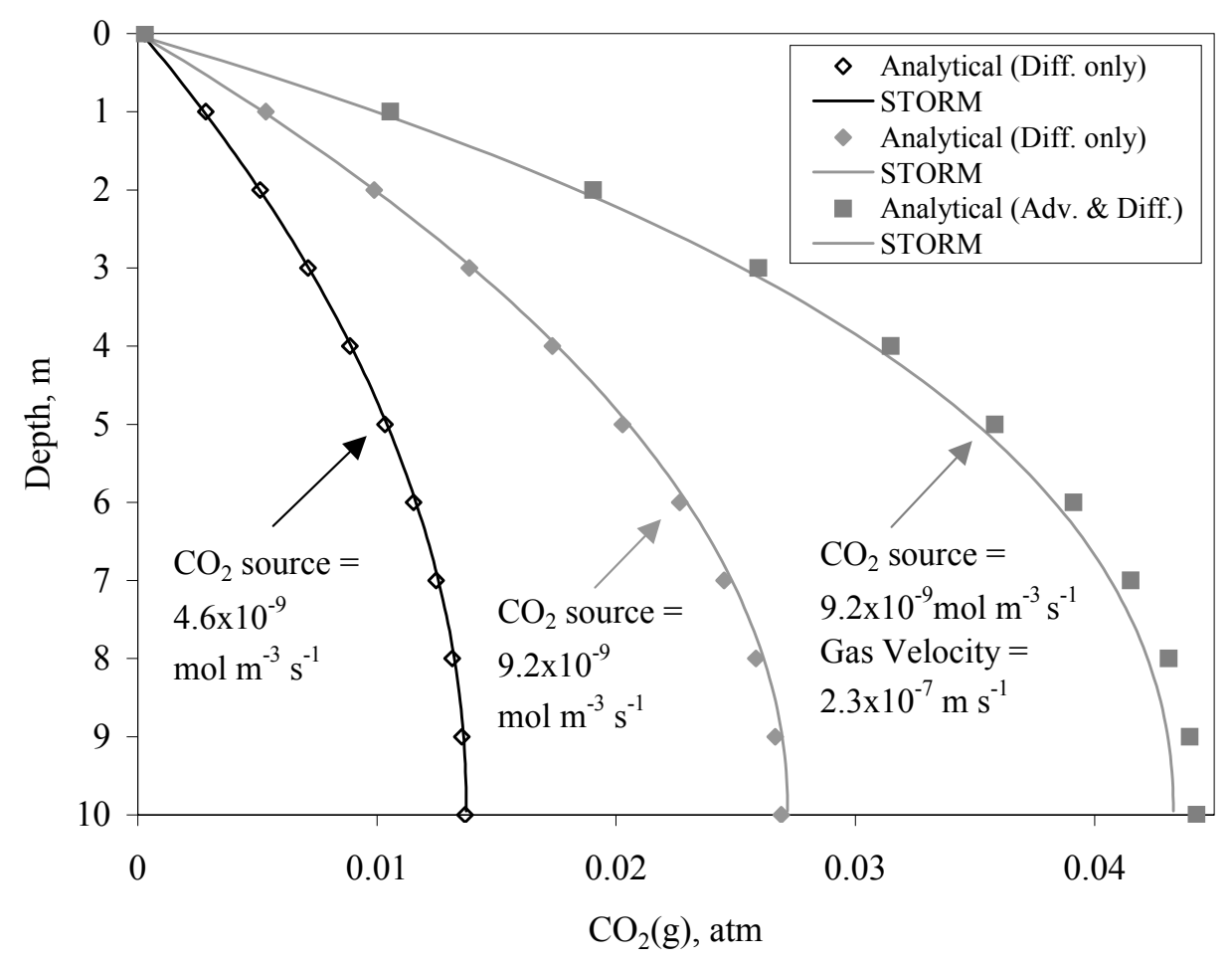

Figure 8.2. Results of Gas Phase Diffusion and Advection Test Cases, Comparing STORM with Analytical Solutions for Constant Gas-Phase Production and Diffusion with and Without Advection 


\subsubsection{Gas Phase Advection and Diffusion}

For the case of 1-dimensional, steady state diffusion and advection of a single gas species, with constant production from a single reaction with depth, Equation (2.17) simplifies to

$$
W=-\left(\theta_{g} \mathbf{D}^{g} K_{H}^{\prime-1}+\theta_{a} \mathbf{D}^{a}\right) \frac{\partial^{2} C^{a}}{\partial z^{2}}+\left(\theta_{g} \mathbf{V}_{g} K_{H}^{\prime-1}+\theta_{a} \mathbf{V}_{a}\right) \frac{\partial C^{a}}{\partial z}
$$

Assuming production occurs over a finite depth $0<z<L$, with a specified concentration $\left(C_{0}^{a}\right)$ at the top boundary $(z=0)$ and no diffusive flux at the lower boundary $(z=L)$, the aqueous phase concentration of that gas species is given by

$$
C_{z}^{a}=\frac{\mathbf{D}\left(\mathrm{e}^{\frac{L \mathbf{v}}{\mathbf{D}}}-\mathrm{e}^{\frac{(L-z) \mathbf{v}}{\mathbf{D}}}\right) W+\mathbf{v}\left(C_{0}^{a} \mathbf{v}-W z\right)}{\mathbf{v}^{2}}
$$

where $\mathbf{V}=\theta_{g} \mathbf{V}_{g} K_{H}^{\prime-1}+\theta_{a} \mathbf{V}_{a}$ and $\mathbf{D}=\theta_{g} \mathbf{D}^{g} K_{H}^{\prime-1}+\theta_{a} \mathbf{D}^{a}$. The gas phase concentration may be determined using Equation (2.16). A STORM simulation was run that corresponds to this analytical solution. The model parameters are listed in Table 8.2. The results are shown in green in Figure 8.2. Results for STORM and the analytical solution again agree quite well. The upward gas velocity of $2.3 \times 10^{-7} \mathrm{~m} \mathrm{~s}^{-1}$ increases the $\mathrm{CO}_{2}(\mathrm{~g})$ concentrations found at any given depth, as compared to a similar test case without gas advection (Figure 8.2, square symbols).

\subsection{Quartz Dissolution}

The chemical reaction solver of STORM is verified by comparing STORM results to EQ3/6. In STORM, advective and diffusive transport were made negligible by assuming a very low hydraulic conductivity for the porous media and very small aqueous diffusion coefficients for aqueous species. As an example of a simple kinetic reaction path, quartz sand reacts at $100^{\circ} \mathrm{C}$ with deionized water according to the reaction

$$
\text { Quartz } \rightleftarrows \mathrm{SiO}_{2}(a q)
$$

Quartz dissolves according to the $\mathrm{pH}$-independent rate law (Rimstidt and Barnes 1980)

$$
r_{q t z}=A_{S} k\left(1-\frac{Q}{K}\right)
$$


where $r_{q t z}$ is the dissolution rate $\left(\mathrm{mol} \mathrm{s}^{-1}\right), A_{s}$ is the surface area of the mineral $\left(500 \mathrm{~m}^{2}\right), k$ is the reaction rate constant $\left(2 \times 10^{-11} \mathrm{~mol} \mathrm{~m}^{-2} \mathrm{~s}^{-1}\right), K$ is the equilibrium coefficient $\left(10^{-3.9993}\right.$ at $\left.100^{\circ} \mathrm{C}\right)$, and $Q$ is the activity product, in this case equal to the concentration of $\mathrm{SiO}_{2}(\mathrm{aq})$.

After 8 days, $1 \mathrm{~kg}$ of water is saturated with $\mathrm{SiO}_{2}(\mathrm{aq})$ (Figure 8.3). The results from EQ3/6 and STORM are nearly identical.

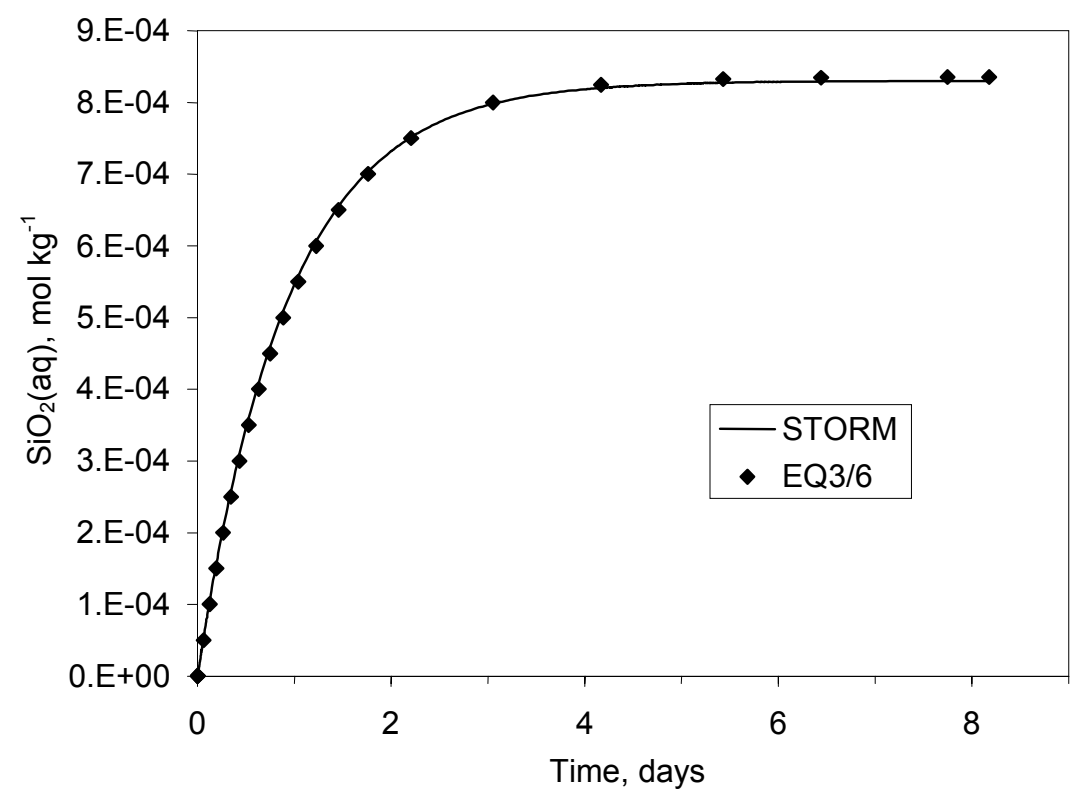

Figure 8.3. Aqueous Silica Concentration as Quartz Dissolves to Equilibrium in Deionized Water at $100^{\circ} \mathrm{C}$

\subsection{Calcite Precipitation}

A more complex kinetic reaction path is used in this verification example, where calcite precipitates from supersaturated solution at $25^{\circ} \mathrm{C}$ according to the reaction

$$
\text { Calcite }+\mathrm{H}^{+} \rightleftarrows \mathrm{Ca}^{2+}+\mathrm{HCO}_{3}^{-}
$$

Calcite dissolves according to the rate law (Reddy et al. 1981):

$$
r_{\text {calcite }}=A_{s} k\left(1-\frac{Q}{K}\right)
$$


where $r_{\text {calcite }}$ is the dissolution rate $\left(\mathrm{mol} \mathrm{s}^{-1}\right), A_{s}$ is the surface area of the mineral $\left(0.24168 \mathrm{~m}^{2}\right), k$ is the reaction rate constant $\left(7 \times 10^{-7} \mathrm{~mol} \mathrm{~m}^{-2} \mathrm{~s}^{-1}\right), K$ is the equilibrium coefficient $\left(10^{1.8487}\right.$ at $\left.25^{\circ} \mathrm{C}\right)$, and $Q$ is the ion-activity product, in this case equal to

$$
Q=\frac{\left[\mathrm{Ca}^{2+}\right]\left[\mathrm{HCO}_{3}^{-}\right]}{\left[\mathrm{H}^{+}\right]}
$$

Several other species participate in equilibrium reactions (Table 8.3) and are included in the simulations. The $\mathrm{CO}_{2}(\mathrm{~g})$ partial pressure is fixed at a partial pressure of $10^{-1.5421}$ bars. The initial total $\mathrm{Ca}$ concentration is $1.2732 \times 10^{-2} \mathrm{~mol} \mathrm{~kg}^{-1}$ and the initial $\mathrm{pH}$ is 7.5815 .

Precipitation of calcite in $1 \mathrm{~kg}$ of water proceeds rapidly, then virtually ceases after the solution has reached saturation (0.12 days). Results from EQ3/6 and STORM are nearly identical (Figure 8.4).

Table 8.3. Aqueous Species, Equilibrium Reactions, and Equilibrium Constants Related to Calcite Dissolution

\begin{tabular}{||l|c||}
\hline \multicolumn{1}{|c|}{ Reaction } & Log $\mathrm{K}$ at $25^{\circ} \mathrm{C}$ \\
\hline $\mathrm{OH}^{-}+\mathrm{H}^{+} \leftrightarrow \mathrm{H}_{2} \mathrm{O}$ & 13.9951 \\
\hline $\mathrm{CO}_{2}(a q)+\mathrm{H}_{2} \mathrm{O} \leftrightarrow \mathrm{H}^{+}+\mathrm{HCO}_{3}^{-}$ & -6.3447 \\
\hline $\mathrm{CO}_{2}(g) \leftrightarrow \mathrm{CO}_{2}(a q)$ & -1.4689 \\
\hline $\mathrm{CaHCO}_{3}^{+} \leftrightarrow \mathrm{Ca}^{2+}+\mathrm{HCO}_{3}^{-}$ & -1.0467 \\
\hline $\mathrm{CaCO}_{3}(a q)+\mathrm{H}^{+} \leftrightarrow \mathrm{Ca}^{2+}+\mathrm{HCO}_{3}^{-}$ & 7.0017 \\
\hline $\mathrm{CO}_{3}^{2-}+\mathrm{H}^{+} \leftrightarrow \mathrm{HCO}_{3}^{-}$ & 10.3288 \\
\hline $\mathrm{CaOH}^{+}+\mathrm{H}^{+} \leftrightarrow \mathrm{Ca}^{2+}+\mathrm{H}_{2} \mathrm{O}$ & 12.8500 \\
\hline
\end{tabular}




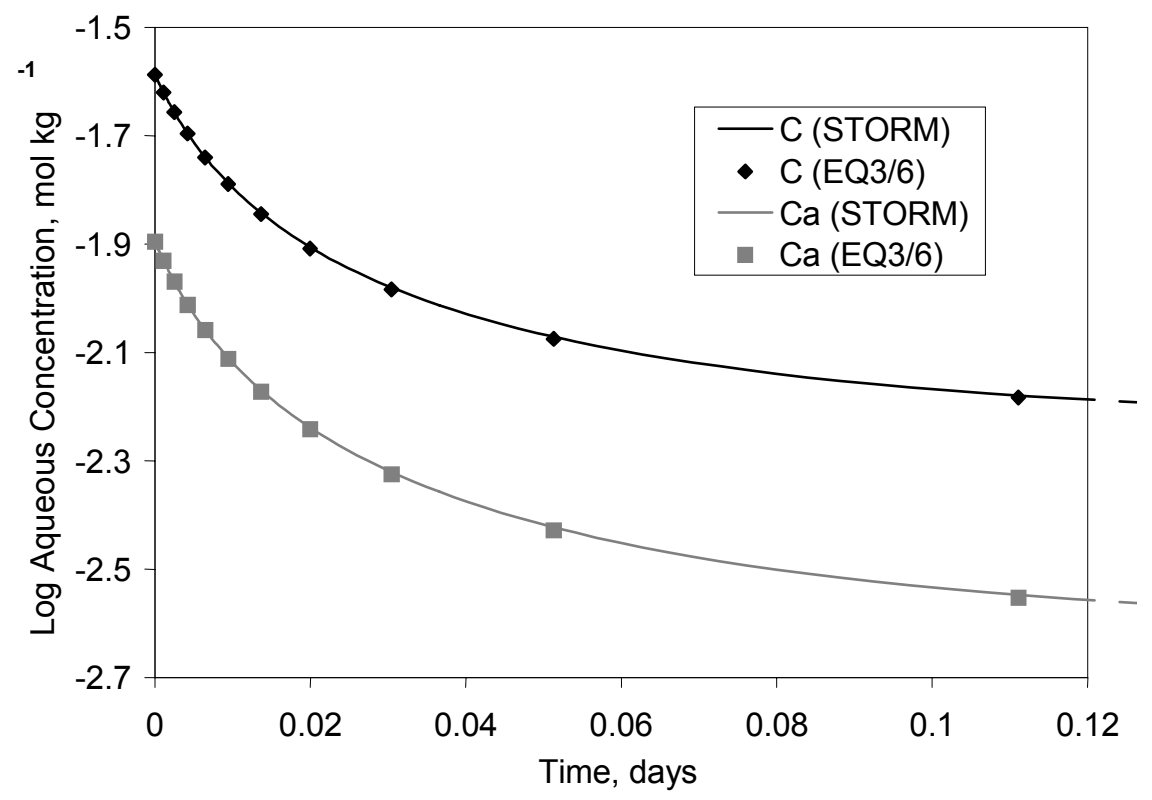

Figure 8.4. Total $\mathrm{C}$ and $\mathrm{Ca}$ Aqueous Concentrations as Calcite Precipitates from Supersaturated Solution 


\subsection{Example Simulation}

This simulation is presented an example of a large, complex problem, that is too large to be run on a conventional workstation, and must be run in parallel. A large inventory of radioactive and mixed waste is currently stored in 177 buried single- and double-shell tanks at the U.S. Department of Energy's (DOE) Hanford Site in southeastern Washington State. Liquid waste recovered from the tanks will be pretreated to separate the low activity fraction, which will be immobilized in glass at a waste treatment plant (WTP) and placed in a near-surface integrated disposal facility (IDF) (Mann et al. 2003). This simulation estimates the dissolution rate of LAWABP1 waste glass blocks buried in backfill soil, and calculates the flux of Technetium across the lower model boundary, which is used to estimate a potential drinking-water dose. The results of parallel scaleup tests for this example problem are also presented.

\subsection{Simulation Parameters}

Simulations were based on the latest conceptual design (Mann et al. 2003) of the IDF subsurface disposal facility trench (Figure 9.1). Because the trench is 764 feet wide, only a single stack of waste packages was modeled (Figure 9.2); this model incorporates the assumption that the trench is essentially infinite in the horizontal plane. The modeled domain was $1.52 \mathrm{~m}$ wide by $17.8 \mathrm{~m}$ tall, with 76 nodes in the horizontal direction and 890 nodes in the vertical direction. Three material zones are included in the simulation, waste glass, backfill in the trench surrounding the waste glass, and Hanford Sand below the trench. There is a sharp contrast between the unsaturated hydraulic properties of the waste glass, a fractured medium, and the surrounding backfill, a porous medium (Table 9.1).

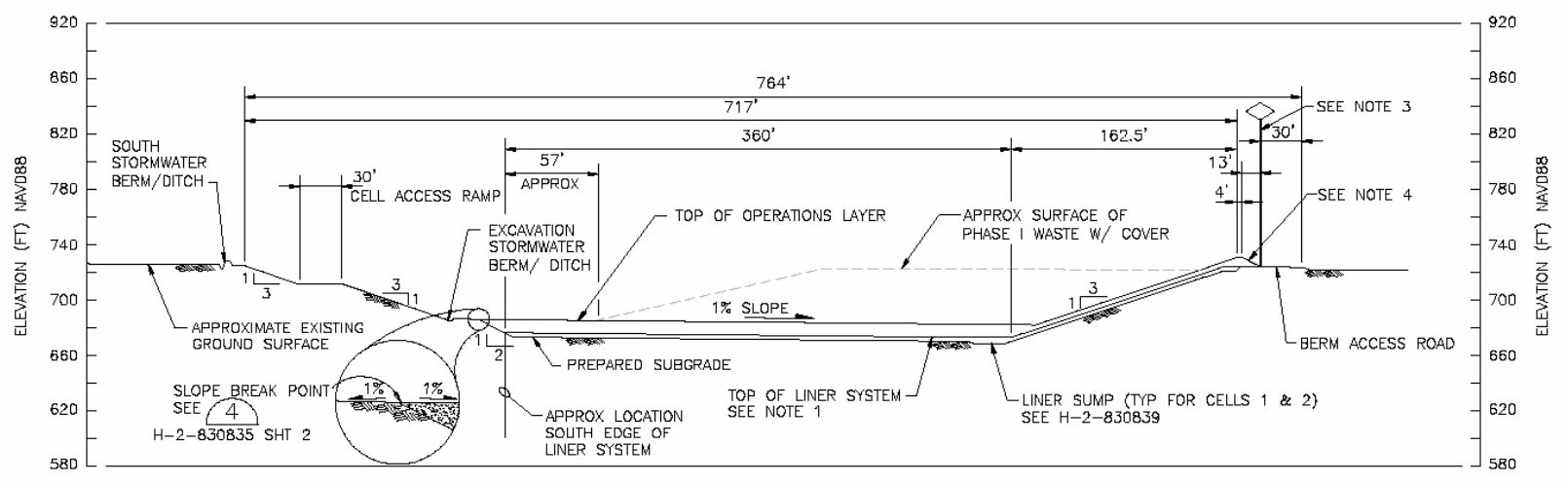

Figure 9.1. Cross Section of IDF Trench

The corrosion reaction for LAWABP1 glass (McGrail et al. 2000) used in the simulation is:

$$
\begin{aligned}
\text { LAWABP } 1+4.42 \times 10^{-1} \mathrm{H}^{+}+1.89 \times 10^{-1} \mathrm{H}_{2} \mathrm{O} \rightarrow 1.36 \times 10^{-1} \mathrm{AlO}_{2}^{-}+1.84 \times 10^{-1} \mathrm{~B}(\mathrm{OH})_{3}(\mathrm{aq}) \\
+1.13 \times 10^{-2} \mathrm{Cl}^{-}+1.82 \times 10^{-4} \mathrm{CrO}_{4}^{2-}+1.46 \times 10^{-3} \mathrm{~F}^{-}+2.16 \times 10^{-2} \mathrm{Fe}(\mathrm{OH})_{3}(\mathrm{aq}) \\
+3.23 \times 10^{-2} \mathrm{~K}^{+}+8.48 \times 10^{-3} \mathrm{La}^{3+}+1.71 \times 10^{-2} \mathrm{Mg}^{2+}+4.46 \times 10^{-1} \mathrm{Na}^{+} \\
+7.79 \times 10^{-4} \mathrm{HPO}_{4}^{2-}+8.63 \times 10^{-4} \mathrm{SO}_{4}^{2-}+4.82 \times 10^{-1} \mathrm{SiO}_{2}(\mathrm{aq})+6.59 \times 10^{-7} \mathrm{TcO}_{4}^{-} \\
+2.15 \times 10^{-2} \mathrm{Ti}(\mathrm{OH})_{4}(\mathrm{aq})+2.20 \times 10^{-2} \mathrm{Zn}^{2+}+2.94 \times 10^{-2} \mathrm{Zr}(\mathrm{OH})_{4}(\mathrm{aq})
\end{aligned}
$$




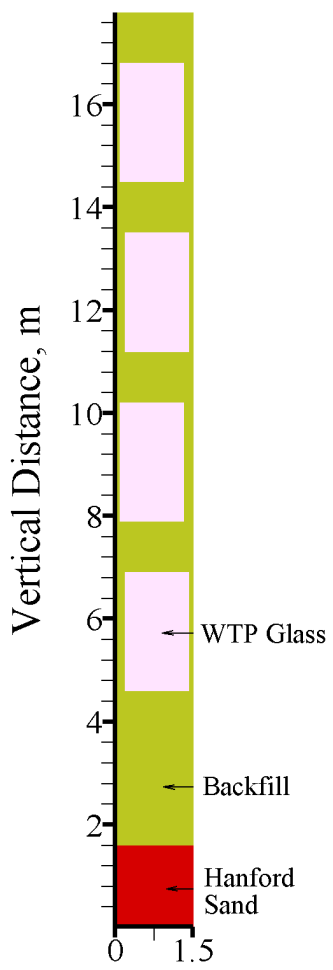

Horizontal Distance, $m$

Figure 9.2. Material Zonation for WTP Glass Waste Form Release Simulation

Table 9.1. Material Hydraulic Properties Used In Simulations (Meyer and Serne 1999)

\begin{tabular}{|l|c|c|c|c|c|c||}
\hline \multicolumn{1}{|c|}{ Material } & $\begin{array}{c}\text { Particle } \\
\text { Density } \\
\left(\mathbf{g} / \mathbf{c m}^{-3}\right)\end{array}$ & $\begin{array}{c}\text { Saturated } \\
\text { Water } \\
\text { Content }\end{array}$ & $\begin{array}{c}\text { Residual } \\
\text { Water } \\
\text { Content }\end{array}$ & $\begin{array}{c}\alpha\left(\mathbf{c m}^{-1}\right)(\mathbf{v a n} \\
\text { Genuchten 1980) }\end{array}$ & $\begin{array}{c}\text { n (van } \\
\text { Genuchten } \\
\mathbf{1 9 8 0})\end{array}$ & $\begin{array}{c}\text { Saturated Hydraulic } \\
\text { Conductivity } \\
\left(\mathbf{c m} / \mathbf{s}^{-1}\right)\end{array}$ \\
\hline Waste Glass & 2.68 & 0.020 & 0.00046 & 0.200 & 3.00 & $1.00 \times 10^{-2}$ \\
\hline Backfill & 2.76 & 0.316 & 0.049 & 0.035 & 1.72 & $1.91 \times 10^{-3}$ \\
\hline Hanford Sand & 2.63 & 0.375 & 0.041 & 0.055 & 1.77 & $2.88 \times 10^{-3}$ \\
\hline
\end{tabular}

Technetium is the only radionuclide considered in this example; it acts as a tracer in this environment and therefore gives an estimate of the maximum potential radionuclide release rate from the glass. The stoichiometric coefficients for technetium are based on the anticipated average waste package concentration (Wootan 1999). The normalized technetium release rate was calculated by summing the technetium release rate at each node in the model, and dividing by the total amount of technetium (in the glass) in the model domain at the start of the simulation.

Glass dissolution is assumed controlled by the following kinetic rate law:

$$
r_{g}=\vec{k} a_{\mathrm{H}^{+}}^{-\eta} e \frac{-E_{a}}{\mathrm{RT}}\left[1-\frac{a_{\mathrm{SiO}_{2}(\mathrm{aq})}}{K_{g}}\right]
$$


where

$$
\begin{array}{ll}
\mathrm{r}_{\mathrm{g}} & =\text { dissolution rate, } \mathrm{mol} \mathrm{m} \mathrm{s}^{-1} \\
\vec{k} & =\text { intrinsic rate constant, } 0.17 \mathrm{~mol} \mathrm{~m}^{-2} \mathrm{~s}^{-1} \\
a_{\mathrm{H}^{+}} & =\text {hydrogen ion activity (variable calculated by STORM) } \\
\eta & =\text { pH power law coefficient (neutral to basic), } 0.5 \\
\mathrm{E}_{\mathrm{a}} & =\text { activation energy, } 75 \mathrm{~kJ} \mathrm{~mol}^{-1} \\
\mathrm{R} & =\text { gas constant, } 8.134 \times 10^{-3} \mathrm{~kJ} \mathrm{~mol}^{-1} \mathrm{~K}^{-1} \\
\mathrm{~T} & =\text { temperature, } \mathrm{K}\left(\text { assumed constant at } 15^{\circ} \mathrm{C}\right) \\
a_{\mathrm{SiO}_{2}(\mathrm{aq})} & =\text { aqueous silica activity (variable calculated by } \mathrm{STORM} \\
\mathrm{K}_{\mathrm{g}} & =\text { pseudoequilibrium constant for glass, based on } \mathrm{SiO}_{2}(\mathrm{aq}) \text { activity, } 10^{-2.853}
\end{array}
$$

Eq. (9.2) is an approximation for glass because glass is metastable, and the reaction proceeds one way (i.e., glass dissolves). The unknown parameters in Eq. (9.1) $\left(\vec{k}, E_{a}, K_{g}\right.$, and $\left.\eta\right)$ are based on experimental data (McGrail et al. 2000). The mass density of LAWABP1 glass is $2.68 \mathrm{~g} \mathrm{~cm}^{-3}$.

An empirical model was used to calculate the diffusion coefficient for each aqueous species, based on experimental data for the backfill and Hanford Sand (Meyer and Serne 1999):

$$
D_{i}=1.486 D_{f} \theta^{1.956}
$$

where $D_{f}$ is the free water diffusion coefficient and $\theta$ is the water content. The dispersivity was assumed to be $1 \mathrm{~cm}$ based on the $2 \mathrm{~cm}$ grid size of the model to restrict the Peclet number (Bear 1979) to a maximum value of 2 . In addition, test results with LAWABP1 glass (and most other ILAW glasses) show that it was susceptible to a secondary reaction mechanism, alkali ion exchange (McGrail et al. 2001). This reaction resulted in the selective extraction of sodium via the reaction

$$
\text { LAWABP1-Na }+\mathrm{H}^{+} \rightarrow \mathrm{LAWABP} 1-\mathrm{H}+\mathrm{Na}^{+}
$$

where LAWABP1-Na represents the unreacted glass containing sodium and LAWABP1-H represents a hydrated glass where the sodium has been replaced with an equimolar amount of hydrogen. The rate of this reaction has been determined to be $4 \times 10^{-11} \mathrm{~mol} \mathrm{~m}^{-2} \mathrm{~s}^{-1}$ from single-pass flow-through experiments (McGrail et al. 2001). STORM keeps track of the amount of hydrated glass formed via Eq. (9.4) and then allows it to dissolve according to the same kinetic rate law, Eq. (9.2), as the parent glass.

The precipitation of secondary minerals, such as herschelite, lowers the amount of aqueous silica, which can affect the rate of glass dissolution. The secondary minerals likely to precipitate in the glass and surrounding backfill (Table 9.2) were determined by simulating the dissolution of LAWABP1 glass in deionized water at $15^{\circ} \mathrm{C}$ with the $\mathrm{EQ} 3 / 6$ code package (Wolery and Daveler 1992) and the data0.com.R8 database (Daveler and Wolery 1992; Wolery and Daveler 1992). Furthermore, aqueous equilibrium reactions involving sodium and other dissolved glass constituents increase the $\mathrm{pH}$, and hence the rate of glass dissolution, Eq. (9.2). The aqueous species in equilibrium with those produced by the glass dissolution reaction (Table 9.3) were also identified from the EQ3/6 simulations. 
Table 9.2. Secondary Mineral Reaction Network for LAWABP1 Glass

\begin{tabular}{|l|r||}
\hline \multicolumn{1}{|c|}{ Secondary Mineral Reaction } & Log K (15 \\
\hline & $\mathbf{C})$ \\
\hline Analcime $=0.96 \mathrm{AlO}_{2}{ }^{-}+0.96 \mathrm{Na}^{+}+2.04 \mathrm{SiO}_{2}(\mathrm{aq})$ & -9.86 \\
\hline Anatase $+2 \mathrm{H}_{2} \mathrm{O}=\mathrm{Ti}(\mathrm{OH})_{4}(\mathrm{aq})$ & -6.64 \\
\hline Baddeleyite $+2 \mathrm{H}_{2} \mathrm{O}=\mathrm{Zr}(\mathrm{OH})_{4}(\mathrm{aq})$ & -9.29 \\
\hline Gibbsite $=\mathrm{AlO}_{2}{ }^{-}+\mathrm{H}^{+}+\mathrm{H}_{2} \mathrm{O}$ & -13.10 \\
\hline Goethite $+\mathrm{H}_{2} \mathrm{O}=\mathrm{Fe}(\mathrm{OH})_{3}(\mathrm{aq})$ & -11.09 \\
\hline Herschelite $=1.62 \mathrm{Na}^{+}(\mathrm{aq})+0.50 \mathrm{~K}^{+}(\mathrm{aq})+2.26 \mathrm{AlO}_{2}{ }^{-}+4 \mathrm{SiO}_{2}(\mathrm{aq})+0.14 \mathrm{H}^{+}+5.93 \mathrm{H}_{2} \mathrm{O}$ & -40.94 \\
\hline $\mathrm{La}(\mathrm{OH})_{3}(\mathrm{am})+3 \mathrm{H}^{+}=3 \mathrm{H}_{2} \mathrm{O}+\mathrm{La}^{3+}$ & 22.55 \\
\hline Nontronite-Na $+2 \mathrm{H}_{2} \mathrm{O}=0.330 \mathrm{AlO}_{2}{ }^{-}+2 \mathrm{Fe}(\mathrm{OH})_{3}(\mathrm{aq})+0.330 \mathrm{Na}^{+}+3.67 \mathrm{SiO}_{2}(\mathrm{aq})$ & -43.33 \\
\hline Sepiolite $+8 \mathrm{H}^{+}=4 \mathrm{Mg}^{2+}+6 \mathrm{SiO}_{2}(\mathrm{aq})+11 \mathrm{H}_{2} \mathrm{O}$ & 31.29 \\
\hline SiO & -2.85 \\
\hline $\mathrm{Hydr}_{2}(\mathrm{am})=\mathrm{SiO}_{2}(\mathrm{aq})$ & 30.31 \\
\hline
\end{tabular}

Table 9.3. Equilibrium Reactions Considered in WTP Glass Simulation with Equilibrium Coefficients $(\mathrm{K})$ at $15^{\circ} \mathrm{C}$

\begin{tabular}{|c|c|}
\hline Reaction & Log K \\
\hline $\mathrm{BO}_{2}^{-}+\mathrm{H}_{2} \mathrm{O}+\mathrm{H}^{+}=\mathrm{B}(\mathrm{OH})_{3}(\mathrm{aq})$ & 9.35 \\
\hline $\mathrm{CO}_{2}(\mathrm{aq})+\mathrm{H}_{2} \mathrm{O}=\mathrm{H}^{+}+\mathrm{HCO}_{3}^{-}$ & -6.42 \\
\hline $\mathrm{CO}_{3}^{2-}+\mathrm{H}^{+}=\mathrm{HCO}_{3}^{-}$ & 10.43 \\
\hline $\mathrm{H}_{2} \mathrm{SiO}_{4}{ }^{2-}+\mathrm{H}^{+}=2 \mathrm{H}_{2} \mathrm{O}+\mathrm{SiO}_{2}(\mathrm{aq})$ & 22.96 \\
\hline $\mathrm{HCrO}_{4}^{-}=\mathrm{CrO}_{4}^{2-}+\mathrm{H}^{+}$ & -6.49 \\
\hline $\mathrm{HSiO}_{3}{ }^{-}+\mathrm{H}^{+}=\mathrm{SiO}_{2}(\mathrm{aq})+\mathrm{H}_{2} \mathrm{O}$ & 10.10 \\
\hline $\mathrm{KOH}(\mathrm{aq})+\mathrm{H}^{+}=\mathrm{H}_{2} \mathrm{O}+\mathrm{K}^{+}$ & 14.46 \\
\hline $\mathrm{LaCO}_{3}^{+}+\mathrm{H}^{+}=\mathrm{HCO}_{3}^{-}+\mathrm{La}^{3+}$ & 9.13 \\
\hline $\mathrm{MgB}(\mathrm{OH})_{4}{ }^{+}+\mathrm{H}^{+}=\mathrm{H}_{2} \mathrm{O}+\mathrm{B}(\mathrm{OH})_{3}(\mathrm{aq})+\mathrm{Mg}^{2+}$ & 7.35 \\
\hline $\mathrm{MgCO}_{3}(\mathrm{aq})+\mathrm{H}^{+}=\mathrm{HCO}_{3}^{-}+\mathrm{Mg}^{2+}$ & 7.50 \\
\hline $\mathrm{MgHCO}_{3}^{+}=\mathrm{HCO}_{3}^{-}+\mathrm{Mg}^{2+}$ & -1.04 \\
\hline $\mathrm{NaB}(\mathrm{OH})_{4}(\mathrm{aq})+\mathrm{H}^{+}=\mathrm{B}(\mathrm{OH})_{3}(\mathrm{aq})+\mathrm{Na}^{+}+\mathrm{H}_{2} \mathrm{O}$ & 8.97 \\
\hline $\mathrm{NaCO}_{3}^{-}+\mathrm{H}^{+}=\mathrm{HCO}_{3}^{-}+\mathrm{Na}^{+}$ & 9.82 \\
\hline $\mathrm{NaHCO}_{3}(\mathrm{aq})=\mathrm{HCO}_{3}^{-}+\mathrm{Na}^{+}$ & -0.24 \\
\hline $\mathrm{NaHSiO}_{3}(\mathrm{aq})+\mathrm{H}^{+}=\mathrm{Na}^{+}+\mathrm{SiO}_{2}(\mathrm{aq})+\mathrm{H}_{2} \mathrm{O}$ & 8.36 \\
\hline $\mathrm{NaOH}(\mathrm{aq})+\mathrm{H}^{+}=\mathrm{Na}^{+}+\mathrm{H}_{2} \mathrm{O}$ & 15.12 \\
\hline $\mathrm{OH}^{-}+\mathrm{H}^{+}=\mathrm{H}_{2} \mathrm{O}$ & 14.34 \\
\hline $\mathrm{ZnOH}^{+}=\mathrm{OH}^{-}+\mathrm{Zn}^{2+}$ & -14.19 \\
\hline $\mathrm{Zn}(\mathrm{OH})_{3}{ }^{-}+3 \mathrm{H}^{+}=3 \mathrm{H}_{2} \mathrm{O}+\mathrm{Zn}^{2+}$ & 28.84 \\
\hline
\end{tabular}

\subsection{Results and Discussion}

A sharp contrast between the unsaturated hydraulic properties of the waste glass (a fractured medium) and the surrounding backfill (a porous medium) causes water to flow around the glass blocks. This lowers both the water content in, and water flux through, the waste packages (Figure 9.4). The glass dissolution rate for these simulations is highest on the edges of the glass layers (Figure 9.3). In these 


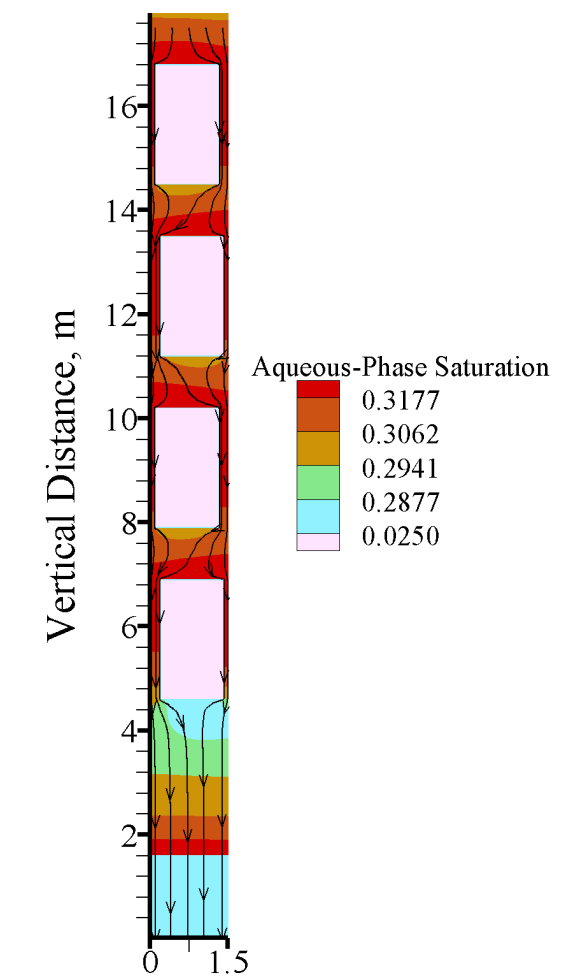

Horizontal Distance, $\mathrm{m}$

Figure 9.4. Simulated Steady-State Water Content and Flow Streamlines in Subsurface Disposal Facility Assuming Recharge Rate of $4.2 \mathrm{~mm} / \mathrm{yr}$

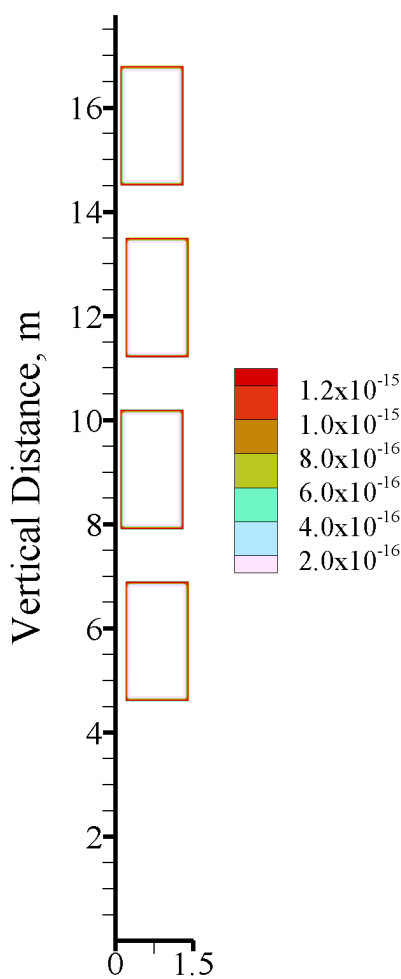

Horizontal Distance, $m$

Figure 9.3. Simulated Waste Glass Dissolution Rate $\left(\mathrm{mole} / \mathrm{m}^{2} / \mathrm{s}\right)$ at $10,000 \mathrm{yr}$

regions the $\mathrm{pH}$ is high (Figure 9.5), and $\mathrm{SiO}_{2}$ (aq) concentrations are low (Figure 9.6) due to diffusion and secondary mineral precipitation in the backfill. Technetium concentrations are high in the glass blocks but are diluted when diffusing into the wetter surrounding backfill (Figure 9.7). Data for the contour plots were taken from plot files output by STORM.

Assuming a water recharge rate of $4.2 \mathrm{~mm} / \mathrm{yr}$ yields a release rate of, $2.8 \times 10^{-8} \mathrm{yr}^{-1}$ (Figure 9.8). The relevant performance objective of the disposal system for protecting groundwater resources is a betaphoton drinking water dose of no more than $4 \mathrm{mrem} \mathrm{yr}^{-1}$ (40 CFR 141 1975). Based on estimated transport to a well $100 \mathrm{~m}$ down gradient of the disposal facility (Mann et al. 2000), at a recharge rate of $4.2 \mathrm{~mm} / \mathrm{yr}$, the maximum allowable technetium flux is $1.7 \times 10^{-4} \mathrm{yr}^{-1}$. The release rates estimated by STORM is 3.8 orders of magnitude less than the maximum allowable technetium release rate. 


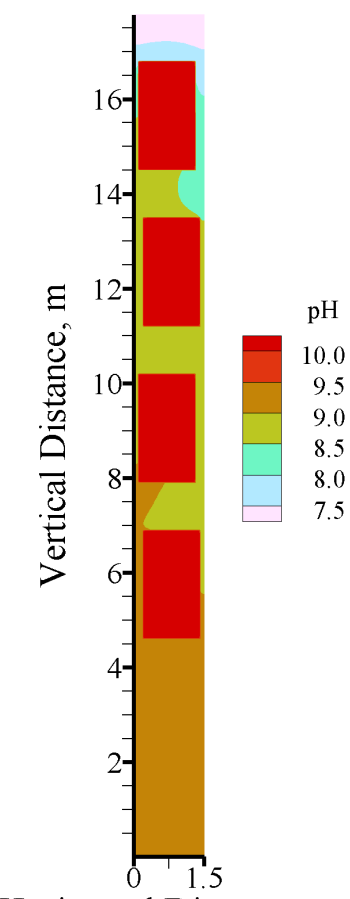

Horizontal Distance, $m$

Figure 9.5. Simulated $\mathrm{pH}$ at $10,000 \mathrm{yr}$

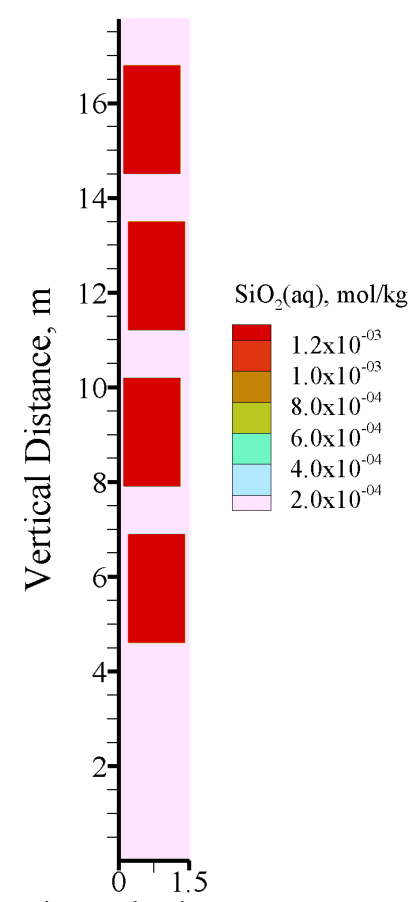

Horizontal Distance, $m$

Figure 9.6. Simulated $\mathrm{SiO}_{2}$ (aq) Concentrations (mole $/ \mathrm{kg}$ ) at $10,000 \mathrm{yr}$

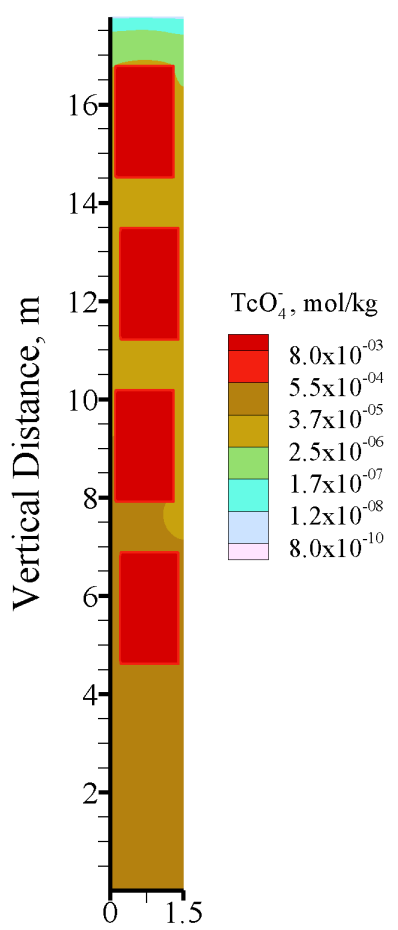

Horizontal Distance, $\mathrm{m}$

Figure 9.7. Simulated Technetium Concentrations (moles $/ \mathrm{kg}$ ) at $10,000 \mathrm{yr}$ 


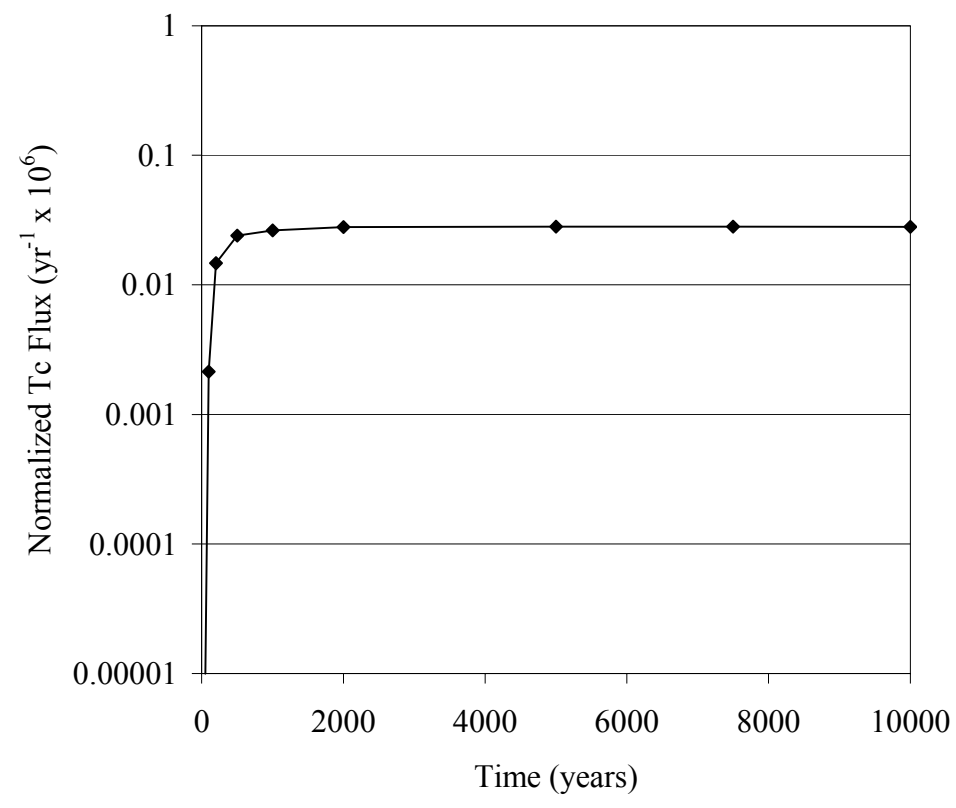

Figure 9.8. Technetium Flux Across Lower Model Boundary Relative to Total Initial Amount of Technetium in Model

\subsection{Parallel Scaleup}

In order to simulate the weathering of the waste glass over 10,000 years with the detail necessary to represent the complex, multidimensional flow through the subsurface disposal system, the simulation described in the previous section was run in parallel on the high-performance computing cluster at the Molecular Science Computing Facility (MSCF) at PNNL.

The simulation described in the previous section was run in parallel on the EMSL mpp2 supercomputer, out to a simulation time of five years. Progressively faster simulation times were achieved on 9 to 256 processors (Figure 9.9). The decrease in run time from 19.6 to $1.3 \mathrm{hr}$ is very good but less than ideal due to the overhead of interprocess communication and redundant calculations. 


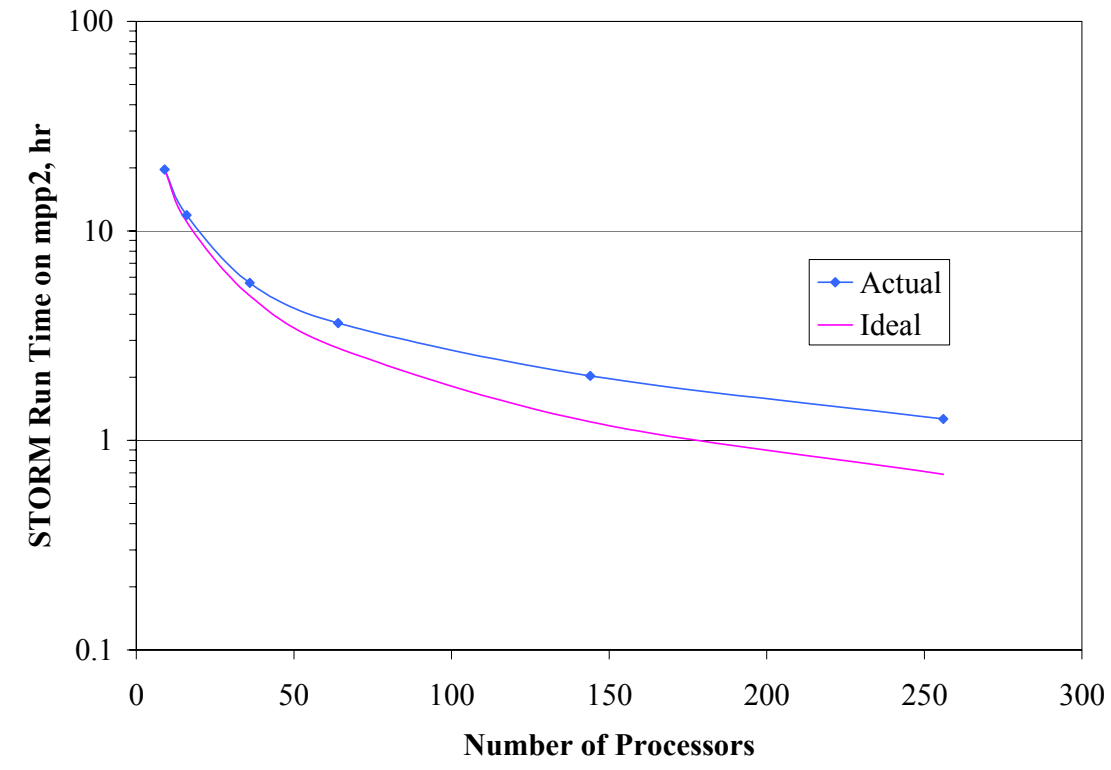

Figure 9.9. Scale-up of Parallel STORM on Multiple, Parallel Processors 


\subsection{References}

40 CFR 141. 1975. National Primary Drinking Water Regulations. U.S. Environmental Protection Agency, Washington, DC.

Barry DA, CT Miller, and PJ Culligan-Hensley. 1996. "Temporal discretization errors in non-iterative split-operator approaches to solving chemical reaction/groundwater transport models." J. Contam. Hydrol., 22:1-17.

Bear J. 1979. Hydraulics of Groundwater. McGraw-Hill, New York.

Bramley $\mathrm{R}$ and X Wang. 1995. A library of iterative methods for sparse linear systems. Indiana University, Bloomington.

Carman PC. 1937. "Fluid flow through a granular bed." Trans. Instn. Chem. Engrs., 15:150-156.

Chen Y, DW Engel, BP McGrail, and KS Lessor. 1995. AREST-CT V1.0 Software Verification. PNL-10692, Pacific Northwest Laboratory, Richland, WA.

Chen Y, P McGrail, and DW Engel. 1996. "A reaction transport model and its application to performance assessment of nuclear waste disposal." International Conference on Deep Geological Disposal of Radioactive Waste, Canadian Nuclear Society.

Chen Y, BP McGrail, and DW Engel. 1997. "Source-term analysis for Hanford low-activity tank waste using the reaction-transport code AREST-CT." Scientific Basis for Nuclear Waste Management XX, Pittsburgh.

Davies CW. 1962. Ion Association. Butterworths, London.

Dongarra JJ, JR Bunch, CB Moler, and GW Stewart. 1979. LINPACK User's Guide. Society of Industrial and Applied Mathematicians (SIAM), Philadelphia.

Dowd K and C Severance. 1998. High Performance Computing. O'Reilly \& Associates, Inc., Sebastopol, CA.

Ecology. 1996. Hanford Facility Agreement and Consent Order, Sixth Amendment, Washington State Department of Ecology, United States Environmental Protection Agency, and United States Department of Energy, Richland, WA.

Fair GM and LP Hatch. 1933. "Fundamental factors governing the streamline flow of water through sand." J. Am. Wat. Wks. Ass., 25:1551-1565.

Golub GH and CF Van Loan. 1983. Matrix Computations. The Johns Hopkins University Press, Baltimore.

Gupta AD, LW Lake, GA Pope, K Sepehrnoori, and MJ King. 1991. "High-resolution monotonic schemes for reservoir fluid flow simulation." In Situ, 15(3):289-317.

Harten A. 1983. "High-resolution schemes for hyperbolic conservation laws." J. Comp. Phys., 49:357.

Helgeson HC. 1969. "Thermodynamics of hydrothermal systems at elevated temperatures and pressures." American Journal of Science, 267:729-804.

Kirkner DJ and H Reeves. 1988. "Multicomponent mass transport with homogeneous and heterogeneous chemical reactions: Effect of chemistry on the choice of numerical algorithm 1. Theory." Water Resour. Res., 24:1719-1729.

Kreyszig E. 1979. Advanced Engineering Mathematics. John Wiley \& Sons, Inc., New York. 
Lichtner PC. 1985. "Continuum model for simultaneous chemical reaction and mass transport in hydrothermal systems." Geochim. Cosmochim. Acta, 49:779-800.

Mangold DC and C-F Tsang. 1991. "A summary of subsurface hydrological and hydrochemical models." Rev. Geophys., 29:51-79.

Mann FM, SH Finfrock, EJ Freeman, RJ Puigh, II, DH Bacon, MP Bergeron, BP McGrail, and SK Wurstner. 2000. White Paper Updating Conclusions of 1998 ILAW Performance Assessment. DOE/ORP-2000-07 Rev. 0, U.S. Department of Energy Office of River Protection, Richland, WA.

Mann FM, KC Burgard, WR Root, RJP II, SH Finfrock, R Khaleel, DH Bacon, EJ Freeman, BP McGrail, SK Wurstner, and PE Lamont. 2001. Hanford Immobilized Low-Activity Waste Performance Assessment: 2001 Version. DOE/ORP-2000-24 Rev. 0, U.S. Department of Energy Office of River Protection, Richland, WA.

Mann FM, BP McGrail, DH Bacon, RJ Serne, KM Krupka, RJ Puigh, R Khaleel, and SH Finfrock. 2003. Risk Assessment supporting the decision on the initial selection of supplemental ILAW technologies. RPP-17675 Rev.0, CH2M Hill, Richland, WA.

McGrail BP and DH Bacon. 1998. Selection of a Computer Code for Hanford Low-Level Waste Engineered-System Performance Assessment. PNNL-10830 Rev. 1, Pacific Northwest National Laboratory, Richland, WA.

McGrail BP, DH Bacon, JP Icenhower, WL Ebert, PF Martin, HT Schaef, and EA Rodriguez. 2000. Waste Form Release Data Package for the 2001 Immobilized Low-Activity Waste Performance Assessment. PNNL-13043 Rev. 1, Pacific Northwest National Laboratory, Richland, WA.

McGrail BP, DH Bacon, JP Icenhower, WL Ebert, PF Martin, HT Schaef, and EA Rodriguez. 2001. Waste Form Release Data Package for the 2001 Immobilized Low-Activity Waste Performance Assessment. PNNL-13043 Rev. 2, Pacific Northwest National Laboratory, Richland, WA.

Meijerink J and $\mathrm{H}$ van der Vorst. 1977. "An iterative solution method for linear systems of which the coefficient matrix is a symmetric M-matrix." Math. Comp., 31:148-162.

Meyer PD and RJ Serne. 1999. Near Field Hydrology Data Package for the Immobilized Low-Activity Waste 2001 Performance Assessment. PNNL-13035, Pacific Northwest National Laboratory, Richland, WA.

Ortoleva P, E Merino, C Moore, and J Chadam. 1987. "Geochemical self-organization II: The reactiveinfiltration instability." Am. J. Sci., 287:1008-1040.

Patankar SV. 1980. Numerical Heat Transfer and Fluid Flow. Hemisphere Publishing Corporation, Washington, DC.

Pitzer KS. 1973. "Thermodynamics of electrolytes - 1. Theoretical basis and general equations." Journal of Physical Chemistry, 4:249-265.

Press WH, SA Teukolsky, WT Vetterling, and BP Flannery. 1986. Numerical Recipes in FORTRAN: The Art of Scientific Computing. Cambridge University Press.

Reddy MM, LN Plummer, and E Busenberg. 1981. "Crystal growth of calcite from calcium bicarbonate solutions at constant $\mathrm{PCO}_{2}$ and $25^{\circ} \mathrm{C}$ : A test of a calcite dissolution model." Geochim. et Cosmochim. Acta, 45:1281-1289.

Reed MH. 1982. "Calculation of multicomponent chemical equilibria and reaction processes in system involving minerals, gases and and aqueous phase." Geochim. Cosmochim. Acta, 46:513-528.

Rimstidt JD and HL Barnes. 1980. "The kinetics of silica-water reactions." Geochim. et Cosmochim. Acta, 44:1683-1699. 
Sevougian SD, RS Schechter, and LW Lake. 1993. "Effect of partial local equilibrium on the propagation of precipitation/dissolution waves." Ind. Eng. Chem. Res., 32:2281-2304.

Steefel CI and AC Lasaga. 1994. "A coupled model for transport of multiple chemical species and kinetic precipitation/dissolution reactions with application to reactive flow in single phase hydrothermal systems.” Am. J. Sci., 294:529-592.

Strikwerda JC. 1989. Finite Difference Schemes and Partial Differential Equations. Chapman \& Hall, New York.

Sweby PK. 1984. "High resolution schemes using flux limiters for hyperbolic conservation laws." SIAM J. Numer. Anal, 21:995.

Talbott S. 1988. Managing Projects with Make. O'Reilly and Associates, Inc., Newton, MA.

Valocchi AJ and M Malmstead. 1992. "Accuracy of operator splitting for advection-dispersion-reaction problems.” Water Resour. Res., 28:1471-1476.

van der Vorst H. 1992. "Bi-CGSTAB: A fast and smoothly converging variant of Bi-CG for the solution of nonsymmetric linear systems." SIAM Journal of Scientific and Statistical Computing, 13:631-644.

van Genuchten MT. 1980. "A closed-form equation for predicting the hydraulic conductivity of unsaturated soils.” Soil Sci. Soc. Am. J., 44:892-898.

White MD and M Oostrom. 1996. STOMP: Subsurface Transport Over Multiple Phases, Theory Guide. PNNL-11217, Pacific Northwest National Laboratory, Richland, WA.

Wolery TJ. 1992. EQ3NR, A Computer Program for Geochemical Aqueous Speciation-Solubility Calculations: Theoretical Manual, User's Guide, and Related Documentation (Version 7.0). UCRL-MA110662 PT III, Lawrence Livermore National Laboratory, Livermore, CA.

Wootan DW. 1999. Immobilized Low-Activity Tank Waste Inventory Data Package. HNF-4921 Rev. 0, Fluor Daniel Northwest, Inc., Richland, WA.

Yeh GT and VS Tripathi. 1989. "A critical evaluation of recent developments in hydrogeochemical transport models of reactive multichemical components." Water Resour. Res., 25:93-108.

Yeh GT and VS Tripathi. 1991. "A model for simulating transport of reactive multispecies components: model development and demonstration." Water Resour. Res., 27:3075-3094.

Yeh T-CJ, R Srivastava, A Guszman, and T Harter. 1993. "A numerical model for water flow and chemical transport in variably saturated porous media." Ground Water, 31:634-644. 


\section{Appendix A}

\section{STORM Version 3.0 Input Data Format}




\section{Appendix A}

\section{STORM Version 3.0 Input Data Format}

\section{A.1 Simulation Title Card}

Card Title $\{\sim$ Simulation [ Title Card ] $\}$

Format: Char

Version Number

Format: Integer,

Simulation Title

Format: Char,

User Name

Format: Char,

Company Name

Format: Char

Input Creation Date

Format: Char

Input Creation Time ${ }^{\mathrm{a}}$

Format: Char $^{a}$,

Number of Simulation Note Lines ${ }^{\mathrm{a}}$

Format: Integer ${ }^{a}$,

For: Number of Simulation Note Lines

Simulation Notes ${ }^{\mathrm{a}}$

Format: Char $^{a}$ (maximum of 132 characters per line)

Endfor: Number of Simulation Note Lines

Endcard: Simulation Title Card 


\section{A.1.1 Simulation Title Card Example}

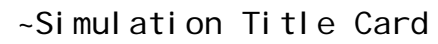

\section{A.2 Solution Control Card}

Card Title $^{\mathrm{a}}\{\sim$ Solution [ Control Card ] $\}$

Format: $\mathrm{Char}^{a}$

Execution Mode Option ${ }^{\mathrm{a}}$

If: Operational Mode Option $=\{$ Water $\}$

$\{$ Normal [ No Flow ] [ Dynamic Domain ] \}

$\{$ Restart[\{ No Flow ] [ Dynamic Domain ] \}

\{ Initial Conditions \}

Else:

\{ Normal \}

\{Restart \}

\{ Initial Conditions \}

Endif:

Format: $\operatorname{Char}^{a}$,

Operational Mode Option $^{\mathrm{a}}$

$\{$ Water $\}$

$\{$ Water-Air

\{Water-Air-Energy

Format: Char $^{a}$,

If: Execution Mode Option $=\{$ Initial Conditions $\}$

Endcard: Solution Control Card

Endif:

Number of Execution Time Periods ${ }^{\mathrm{a}}$

Format: Integer ${ }^{a}$,

For: Number of Execution Time Periods

If: Execution Mode Option $=\{$ Normal $\}$

Initial Time $^{\mathrm{a}}$, Units ${ }^{\mathrm{b}}(\mathrm{s})$, Final Time ${ }^{\mathrm{c}}$, Units $^{\mathrm{d}}(\mathrm{s})$,

Initial Time Step ${ }^{\mathrm{e}}$, $\operatorname{Units}^{\mathrm{f}}(\mathrm{s})$,

Maximum Time Step ${ }^{\mathrm{g}}$, Units ${ }^{\mathrm{h}}$ (s),

Time Step Acceleration Factor, 
Maximum Number of Newton-Raphson Iterations,

Convergence Criterion ${ }^{\mathrm{k}}$

Format: Real ${ }^{a}$, Char $^{b}$, Real $^{c}$, Char $^{d}$, Real $^{e}$, Char $^{f}$, Real $^{g}$, Char $^{h}$, Real $^{i}$, Integer $^{j}$, Real $^{k}$,

ElseIf: Execution Mode Option $=\{$ Restart $\}$

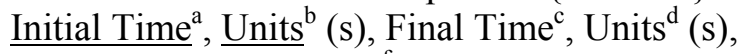

$\underline{\text { Initial Time Step }}^{\mathrm{e}}, \underline{\text { Units }}^{\mathrm{f}}(\mathrm{s})$,

Maximum Time Step ${ }^{\mathrm{g}}$, Units ${ }^{\mathrm{h}}$ (s),

Time Step Acceleration Factori,

Maximum Number of Newton-Raphson Iterations ${ }^{\mathrm{j}}$,

Convergence Criterion $^{\mathrm{k}}$

Format: $\underline{\text { Real }}^{a}, \underline{\text { Char }^{b}}$, Real $^{c}$, Char $^{d}, \underline{\text { Real }}^{e}, \underline{\text { Char }}{ }^{f}, \underline{\text { Real }}^{g}, \underline{\text { Char }}^{h}, \underline{\text { Real }}^{i}, \underline{\text { Integer }}^{j}, \underline{\text { Real }}^{k}$,

Endif:

Endfor: Number of Execution Time Periods

Maximum CPU Time ${ }^{\mathrm{a}}$, Units ${ }^{\mathrm{b}}$ (s),

Maximum Clock Time ${ }^{c}$, Units ${ }^{\mathrm{d}}(\mathrm{s})$,

Maximum Number of Time Steps ${ }^{\mathrm{e}}$

Format: Real ${ }^{a}$, Char $^{b}$, Real $^{c}$, Char $^{d}$, Integer ${ }^{e}$,

If: Operational Mode Option $=\{$ Water-Air $\}\{$ Water-Air-Energy $\}$

Aqueous Diffusion Option ${ }^{\mathrm{a}}$

\{Zero $\}$

\{ Constant

\{Variable

If: Aqueous Diffusion Option $=\{$ Constant $\}$

Dissolved Air Diffusion Coefficient ${ }^{\mathrm{b}}$, Unit $^{\mathrm{c}}\left(\mathrm{m}^{2} / \mathrm{s}\right)$

Format: Char $^{a}$, Real ${ }^{b}$, Char ${ }^{c}$,

Else:

Format: Char $^{a}$,

Endif:

Endif:

If: Operational Mode Option $=\{$ Water-Air $\}\{$ Water-Air-Energy $\}$

Gas Diffusion Option ${ }^{\mathrm{b}}$

\{Zero \}

\{ Constant

\{ Variable

\{Enhanced \}

If: Gas Diffusion Option $=\{$ Constant $\}$

Water Vapor Diffusion Coefficient ${ }^{\mathrm{b}}, \mathrm{Unit}^{\mathrm{c}}\left(\mathrm{m}^{2} / \mathrm{s}\right)$

Format: Char ${ }^{a}$, Real ${ }^{b}$, Char ${ }^{c}$,

Elseif: Gas Diffusion Option $=\{$ Enhanced $\}$

Clay Mass Fraction ${ }^{\mathrm{b}}$

Format: $\mathrm{Char}^{a}$, Real ${ }^{b}$,

\section{Endif:}

Endif: 
Number of Interfacial Averaging Variables ${ }^{\mathrm{a}}$

Format: Integer ${ }^{a}$,

For: Number of Interfacial Averaging Variables

Surface Variable Option ${ }^{\mathrm{a}}$

\{Aqueous Density $\}$ \{ Aqueous Relative Permeability

\{Aqueous Viscosity \} \{ Dissolved Air Diffusion \}

\{ Gas Density \} \{ Gas Relative Permeability

\{Gas Viscosity \} \{ Hydraulic Dispersion \}

\{ntrinsic Permeability $\}$ Thermal Conductivity

\{Water Vapor Diffusion \} \{Solute Diffusion\}

Interfacial Averaging Scheme Option ${ }^{\mathrm{b}}$

\{Harmonic \} \{ Geometric \} Arithmetic $\}$ \{Upwind

Format: Char ${ }^{a}$, Char $^{b}$,

Endfor: Number of Interfacial Averaging Variables

Endcard: Solution Control Card

\section{A.2.1 Solution Control Card Example}

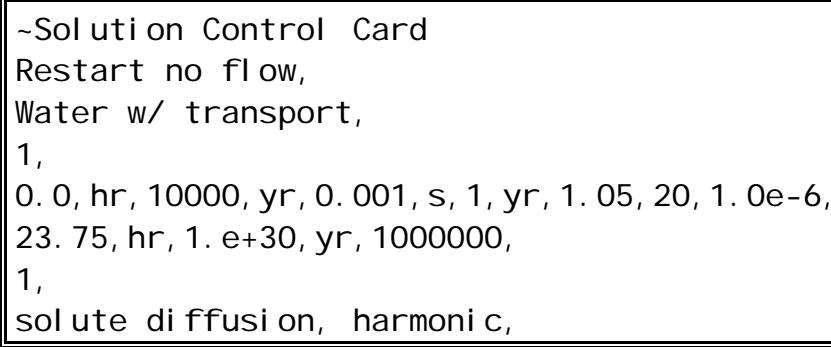

\section{A.3 Grid Card}

Card Title $^{\mathrm{a}}\{\sim$ Grid [ Card ] $\}$

Format: Char $^{a}$

Coordinate System Option ${ }^{\mathrm{a}}$

$\{$ Cartesian

\{Uniform Cartesian \}

Number of X-Dir. Nodes ${ }^{\mathrm{a}}$,

Number of Y-Dir. Nodes ${ }^{\mathrm{b}}$, (note: cannot be greater than 1 in current version)

Number of Z-Dir. Nodes ${ }^{\mathrm{c}}$

Format: Integer ${ }^{a}$, Integer ${ }^{b}$, Integer ${ }^{c}$,

If: Coordinate System Option $=\{$ Cartesian $\}$

For: Number of X-Dir. Nodes

$<$ Surface Position $^{\mathrm{a}}$, Units ${ }^{\mathrm{b}}(\mathrm{m}),>$ or $<$ Count $^{\mathrm{a}} @$ Node Width $^{\mathrm{b}}$, Units $^{\mathrm{c}}(\mathrm{m}),>$

Format: < Real ${ }^{a}$, Char $^{b}$, > or $<$ Integer $^{a} @$ Real $^{a}$, Char $^{b},>$

Endfor: Number of X-Dir. Nodes 


\begin{tabular}{|c|}
\hline 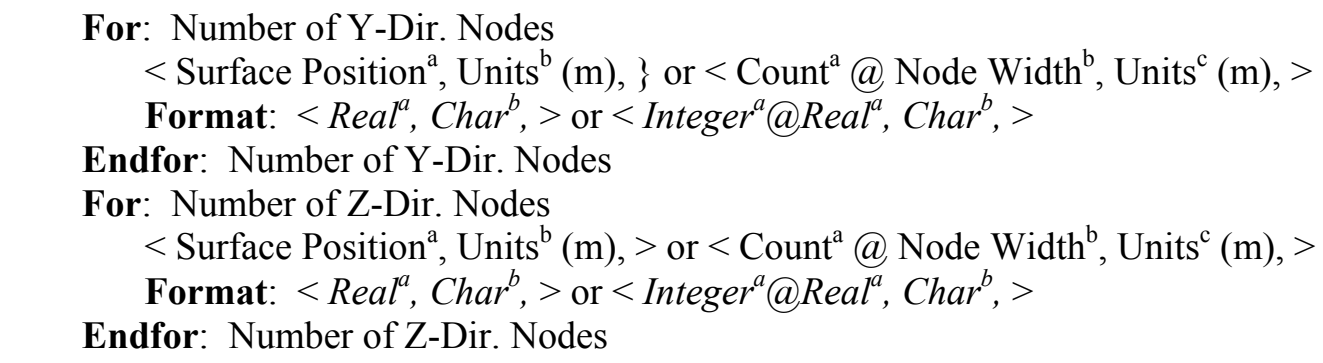 \\
\hline $\begin{array}{l}\text { Elseif: Coordinate System Option }=\{\text { Uniform Cartesian }\} \\
\text { X-Dir. Node Dimension }{ }^{\mathrm{a}}, \text { Units }^{\mathrm{b}}(\mathrm{m}) \\
\text { Format: Real }^{a}, \text { Char }^{b}, \\
\text { Y-Dir. Node Dimension } \\
\text { Format: } \text { Units }^{\mathrm{b}}(\mathrm{m}) \\
\text { Z-Dir. Node Dimension } \\
\text { Z }{ }^{a}, \text { Units }^{\mathrm{b}}(\mathrm{m}) \\
\text { Format: Real }{ }^{a}, \text { Char }^{b},\end{array}$ \\
\hline
\end{tabular}

Endcard: Grid Card

\section{A.3.1 Grid Card Examples}

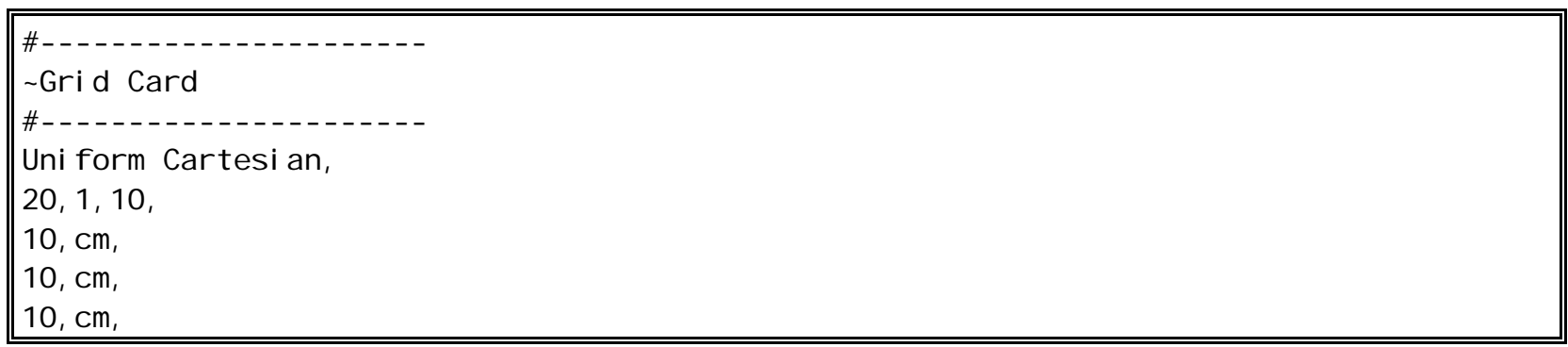

Carid Card

Cartesian,

$76,1,890$,

$0.0, m, 76 @ 0.02, m$,

$0.0, m, 1.0, m$,

$0.0, \mathrm{~m}, 890 @ 0.02, \mathrm{~m}$,

\section{A.4 Inactive Nodes Card}

Card Title $^{\mathrm{a}}\{\sim$ Inactive [ Nodes Card ] $\}$

Format: Char $^{a}$

Number of Inactive Node Domains ${ }^{\mathrm{a}}$

Format: Integer ${ }^{a}$,

For: Number of Inactive Node Domains

I-Start Index ${ }^{\mathrm{a}}$, I-End Index ${ }^{\mathrm{b}}$, 
J-Start Index ${ }^{\mathrm{c}}$, J-End Index ${ }^{\mathrm{d}}$,

$\mathrm{K}$-Start Index ${ }^{\mathrm{e}}, \mathrm{K}$-End Index ${ }^{\mathrm{f}}$

Format: Integer ${ }^{a}$, Integer $^{b}$, Integer $^{c}$, Integer ${ }^{d}$, Integer $^{e}$, Integer $^{f}$,

Endfor: Number of Inactive Node Domains

Endcard: Inactive Nodes Card

\section{A.4.1 Inactive Nodes Card Examples}

I nactive Nodes Card

4 ,

$1,1,1,1,14,113$,

$2,2,1,1,14,33$,

$2,2,1,1,101,113$,

$2,2,1,1,70,70$,

\#-........

$\sim$ nactive

$\#$.........

1 ,

$2,2,1,1,14,20$

$\#$

u nactive Nodes Card

\#

0 , no inactive nodes

\section{A.5 Boundary Conditions Card}

Card Title $^{\text {a }}\{\sim$ Boundary [ Conditions Card ] $\}$

Format: Char $^{a}$

Number of Boundary Condition Domains ${ }^{\mathrm{a}}$,

Format: Integer ${ }^{a}$,

For: Number of Boundary Condition Domains

Boundary Surface Direction Option ${ }^{\mathrm{a}}$,

$\{$ Bottom $\}$ \{ South $\}\{$ West $\}$ \{ East $\}$ North $\}\{$ Top $\}$

If: Operational Mode Option: Solution Control Card $=\{$ Water $\}$

Aqueous-Phase Boundary Type Option ${ }^{\mathrm{b}}$,

\{Dirichlet \} \{ Neumann \} \{ Zero Flux \} \{ Saturated \} \{ Unit Gradient

\{Free Gradient $\}$ \{ Hydraulic Gradient $\}$ \{ Initial Condition \}

Format: Char $^{a}$, Char $^{b},<$ Char $^{c},>$

Elseif: Operational Mode Option: Solution Control Card $=\{$ Water-Air $\}$

Aqueous-Phase Boundary Type Option ${ }^{\mathrm{b}}$ 


\begin{tabular}{|c||}
\hline \{Dirichlet $\}\{$ Neumann $\}\{$ Zero Flux $\}\{$ Unit Gradient $\}$ \\
\{Hydraulic Gradient $\}\{$ Initial Condition $\}$ \\
Gas-Phase Boundary Type Option \\
\{ Dirichlet $\}$ \{ Neumann $\}\{$ Zero Flux $\}\{$ Unit Gradient $\}$ \\
$\{$ Hydraulic Gradient $\{$ Initial Condition $\}$ \\
Format: Char $^{a}$, Char $^{b}$, Char $^{c},<$ Char $^{d},>$ \\
\hline
\end{tabular}

Elseif: Operational Mode Option: Solution Control Card $=\{$ Water-Air-Energy $\}$

Energy Boundary Type Option ${ }^{\text {b }}$

\{Dirichlet $\}$ \{ Neumann $\}$ \{ Zero Flux

\{Outflow $\{$ Initial Condition

Aqueous-Phase Boundary Type Option ${ }^{c}$

\{Dirichlet $\}$ Neumann $\}$ \{ Zero Flux

\{Unit Gradient \} \{ Hydraulic Gradient \} \{ Initial Condition \}

Gas-Phase Boundary Type Option ${ }^{\mathrm{d}}$

\{Dirichlet $\}$ \{ Neumann $\}$ \{ Zero Flux

\{Unit Gradient \} \{ Hydraulic Gradient \} Initial Condition \}

Format: Char $^{a}$, Char $^{b}$, Char $^{c}$, Char $^{d},<$ Char $^{e},>$

I-Start Index ${ }^{\mathrm{a}}$, I-End Index ${ }^{\mathrm{b}}, \mathrm{J}-$ Start Index $^{\mathrm{c}}$, J-End Index ${ }^{\mathrm{d}}$,

K-Start Index ${ }^{\mathrm{e}}, \mathrm{K}-$ End Index ${ }^{\mathrm{f}}$, Number of Boundary Times ${ }^{\mathrm{g}}$

Format: Integer ${ }^{a}$, Integer $^{b}$, Integer $^{c}$, Integer $^{d}$, Integer $^{e}$, Integer $^{f}$, Integer $^{g}$,

If: Operational Mode Option: Solution Control Card $=\{$ Water $\}$

For: Number of Boundary Times

Boundary Time ${ }^{\mathrm{a}}$, Units ${ }^{\mathrm{b}}$ (s),

If: Aqueous Boundary Type Option $=\{$ Dirichlet $\}\{$ Zero Flux $\}$ Aqueous Pressure ${ }^{\mathrm{c}}$, Units ${ }^{\mathrm{d}}(\mathrm{Pa})$,

Elseif: Aqueous-Phase Boundary Type Option $=\{$ Neumann $\}$

Aqueous Volumetric Flux ${ }^{\mathrm{c}}$, Units ${ }^{\mathrm{d}}(\mathrm{m} / \mathrm{s})$,

Elseif: Aqueous-Phase Boundary Type Option $=\{$ Hydraulic Gradient $\}$

Base Aqueous Pressure ${ }^{c}$, Units $^{\mathrm{d}}(\mathrm{Pa})$,

Else: Null ${ }^{\mathrm{c}}, \mathrm{Null}^{\mathrm{d}}$,

Endif:

Format: Real ${ }^{a}$, Char $^{b}$, Real $^{c}$, Char $^{d}$, Real $^{e}$, Char $^{f}$, Real $^{e}$, Char $^{f},<$ Real $^{e}$, Char $^{f},>$

Endfor: Number of Boundary Times

Elseif: Operational Mode Option: Solution Control Card $=\{$ Water-Air $\}$

For: Number of Boundary Times

Boundary Time ${ }^{\mathrm{a}}$, Units ${ }^{\mathrm{b}}$ (s),

If: Aqueous-Phase Boundary Type Option $=\{$ Dirichlet $\}\{$ Zero Flux $\}$ Aqueous Pressure ${ }^{\mathrm{e}}$, $\mathrm{Units}^{\mathrm{f}}(\mathrm{Pa})$,

Elseif: Aqueous-Phase Boundary Type Option $=\{$ Neumann $\}$

Aqueous Volumetric Flux ${ }^{\mathrm{e}}$, Units ${ }^{\mathrm{f}}(\mathrm{m} / \mathrm{s})$,

Elseif: Aqueous-Phase Boundary Type Option $=\{$ Hydraulic Gradient $\}$

Base Aqueous Pressure ${ }^{\mathrm{e}}$, $\operatorname{Units}^{\mathrm{f}}(\mathrm{Pa})$,

Else: Nulle, Null, 


\begin{tabular}{|l}
\hline Endif: \\
If: Gas-Phase Boundary Type Option $=\{$ Dirichlet $\}\{$ Zero Flux $\}$ \\
Gas Pressure ${ }^{\mathrm{g}}$, Units $^{\mathrm{h}}(\mathrm{Pa})$, \\
Elseif: Gas-Phase Boundary Type Option $=\{$ Neumann $\}$ \\
Gas Volumetric Flux ${ }^{\mathrm{g}}$, Units $^{\mathrm{h}}(\mathrm{m} / \mathrm{s})$, \\
Elseif: Gas-Phase Boundary Type Option $=\{$ Hydraulic Gradient $\}$ \\
Base Gas Pressure ${ }^{\mathrm{g}}$, Units $^{\mathrm{h}}(\mathrm{Pa})$, \\
Else: Null ${ }^{\mathrm{g}}$, Null $^{\mathrm{h}}$, \\
Endif: \\
Water Vapor Relative Humidity \\
Format: Real $^{\mathrm{i}}$, Char $^{b}$, Real $^{c}$, Char $^{d}$, Real $^{e}$, Char $^{f}$, \\
Real $^{g}$, Char $^{h}$, Real $^{g}$, Char $^{h},<$ Real $^{g}$, Char $^{h},>$ \\
Endfor: Number of Boundary Times \\
\hline
\end{tabular}

Elseif: Operational Mode Option: Solution Control Card = $\{$ Water-Air-Energy $\}$

For: Number of Boundary Times

Boundary Time ${ }^{\mathrm{a}}$, Units ${ }^{\mathrm{b}}$ (s),

If: Energy Boundary Type Option $=\{$ Dirichlet $\}$

Temperature ${ }^{\mathrm{c}}$ Units $^{\mathrm{d}}(\mathrm{C})$

Elseif: Energy Boundary Type Option $=\{$ Neumann $\}$

Energy Flux ${ }^{\mathrm{c}}$, Units $^{\mathrm{d}}\left(\mathrm{W} / \mathrm{m}^{\wedge} 2\right)$

Else: Null ${ }^{\mathrm{c}}$, Null ${ }^{\mathrm{d}}$,

Endif:

If: Aqueous-Phase Boundary Type Option $=\{$ Dirichlet $\}$ Zero Flux $\}$ Aqueous Pressure ${ }^{\mathrm{e}}$, Units ${ }^{\mathrm{f}}(\mathrm{Pa})$,

Elseif: Aqueous-Phase Boundary Type Option $=\{$ Neumann $\}$

Aqueous Volumetric Flux ${ }^{\mathrm{e}}$, Units ${ }^{\mathrm{f}}(\mathrm{m} / \mathrm{s})$,

Elseif: Aqueous-Phase Boundary Type Option $=\{$ Hydraulic Gradient $\}$

Base Aqueous Pressure ${ }^{\mathrm{e}}$, Units ${ }^{\mathrm{f}}(\mathrm{Pa})$,

Else: Null ${ }^{\mathrm{e}}$, Null,

Endif:

If: Gas-Phase Boundary Type Option $=\{$ Dirichlet $\}\{$ Zero Flux $\}$

Gas Pressure ${ }^{\mathrm{g}}$, Units ${ }^{\mathrm{h}}(\mathrm{Pa})$,

Elseif: Gas-Phase Boundary Type Option $=\{$ Neumann $\}$

Gas Volumetric Flux ${ }^{\mathrm{g}}$, Units ${ }^{\mathrm{h}}(\mathrm{m} / \mathrm{s})$,

Elseif: Gas-Phase Boundary Type Option $=\{$ Hydraulic Gradient $\}$

Base Gas Pressure ${ }^{\mathrm{g}}$, Units ${ }^{\mathrm{h}}(\mathrm{Pa})$,

Else: Null ${ }^{\mathrm{g}}$, Null ${ }^{\mathrm{h}}$,

Endif:

Water Vapor Relative Humidity ${ }^{\mathrm{i}}$

Format: Real ${ }^{a}$, Char $^{b}$, Real $^{c}$, Char $^{d}$, Real $^{e}$, Char $^{f}$, Real $^{g}$, Char $^{h}$, Real $^{i}$, Reali, Char ${ }^{k}$, Reali, Char ${ }^{k},<$ Real $^{j}, \mathrm{Char}^{k},>$

Endfor: Number of Boundary Times

Endfor: Number of Boundary Condition Domains

Endcard: Boundary Conditions Card

\section{A.5.1 Boundary Conditions Card Examples}




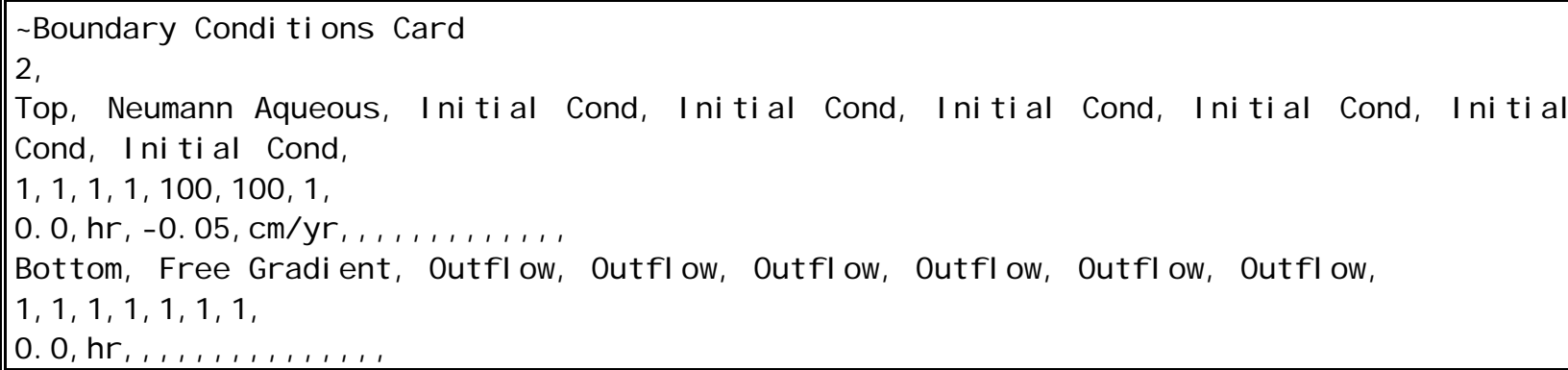

\section{A.6 Output Control Card}

Card Title $^{\mathrm{a}}\{\sim$ Output [ Control Card ] $\}$

Format: Char $^{a}$

Number of Reference Nodes ${ }^{\mathrm{a}}$,

Format: Integer ${ }^{a}$,

For: Number of Reference Nodes

I Index ${ }^{\mathrm{a}}, \mathrm{J}$ Index ${ }^{\mathrm{b}}, \mathrm{K}$ Index ${ }^{\mathrm{c}}$,

Endfor: Number of Reference Nodes

Format: Integer ${ }^{a}$, Integer ${ }^{b}$, Integer ${ }^{c}$,

Reference Node Screen Output Frequency ${ }^{\mathrm{a}}$,

Reference Node Output File Frequency ${ }^{\mathrm{b}}$,

Output Time Units ${ }^{c}$ (s),

Output Length Units ${ }^{\mathrm{d}}(\mathrm{m})$,

Screen Significant Digits ${ }^{\mathrm{e}}$,

Output File Significant Digits ${ }^{\mathrm{f}}$,

Plot File Significant Digits ${ }^{\mathrm{g}}$

Format: Integer ${ }^{a}$, Integer ${ }^{b}$, Char $^{c}$, Char $^{d}$, Integer ${ }^{e}$, Integer $^{f}$, Integer ${ }^{g}$,

Number of Reference Node Variables ${ }^{\mathrm{a}}$,

Format: Integer ${ }^{a}$,

For: Number of Reference Node Variables

Reference Node Variable Option ${ }^{\mathrm{a}}$, Reference Node Variable Units ${ }^{\mathrm{b}}$,

Format: Real ${ }^{a}$, Char $^{b}$,

Endfor: Number of Reference Node Variables

Number of Plot File Times ${ }^{\mathrm{a}}$

Format: Integer ${ }^{a}$,

For: Number of Plot File Times

Plot File Output Time ${ }^{\mathrm{a}}$, Units ${ }^{\mathrm{b}}$ (s)

Format: Real ${ }^{a}$ Char $^{b}$, 
Endfor: Number of Plot File Times

Number of Plot File Variables ${ }^{\mathrm{a}}$

Format: Integer ${ }^{a}$,

For: Number of Plot File Variables

Plot File Variable Option ${ }^{\mathrm{a}}$, Plot File Variable Units ${ }^{\mathrm{b}}$,

Format: Char $^{a}$, Char ${ }^{b}$,

Endfor: Number of Plot File Variables

Note: Refer to the following pages for Plot File Variable Options and Units.

Endcard: Output Control Card

\section{A.6.1 Reference Node Variable and Plot File Variable Options}

If: Operational Mode Option: Solution Control Card $=\{$ Water $\}$

\{apparent water sat [ uration ] \} \{ aqueous gauge pressure \}

$\{$ aqueous moisture cont [ ent ] $\}\{$ water aqueous mass frac [ tion ]\}

\{aqueous pressure $\}$ aqueous sat [ uration ] \}

$\{$ aqueous matrix sat [ uration ] \} \{ aqueous fracture sat [ uration ] \}

$\{$ air aqueous mass frac [ tion ] $\}\{$ aqueous relative perm [ eability ] \}

\{aqueous hydraulic head $\}$ aqueous density

$\{$ water aqueous conc [ entration ] $\}$ \{air aqueous conc [ entration ] \}

\{diffusive porosity $\}\{$ effective trapped air sat [ uration ] \} \{ gas fracture sat [ uration ] \}

$\{$ gas sat [ uration ] $\}\{$ gas pressure $\}\{$ gas matrix sat [ uration ] \}

$\{$ phase condition $\}$ \{rock/soil type $\}$

\{temperature $\}$ \{total water mass

$\{$ water source [ integral ] \}

$\{\mathrm{x}$ aqueous vol [ umetric flux (lower surface) ] \}

$\{$ xnc aqueous vol [ umetric flux (node centered) ] \}

$\{$ y aqueous vol [ umetric flux (lower surface) ] \}

$\{$ ync aqueous vol [ umetric flux (node centered) ] \}

$\{\mathrm{z}$ aqueous vol [ umetric flux (lower surface) ] \}

$\{$ znc aqueous vol [ umetric flux (node centered) ] \}

\section{A.6.2 Reference Node Variable and Plot File Variable Options}

EIseif: Operational Mode Option: Solution Control Card $=\{$ Water-Air $\}$

$\{$ air source [ integral ] $\}\{$ apparent water sat [ uration ] \}

$\{$ air aqueous mass frac [ tion ] $\}\{$ water aqueous mass frac [ tion ] \}

\{aqueous fracture sat [ uration] \} \{aqueous hydraulic head \}

$\{$ aqueous density $\}$ \{aqueous relative perm [ eability ] \}

$\{$ aqueous matrix sat [ uration ] $\}$ \{ air aqueous conc [ entration ] \}

\{aqueous pressure $\}$ aqueous gauge pressure

$\{$ aqueous sat [ uration ] $\}\{$ water aqueous conc [ entration ] \}

$\{$ aqueous moisture cont [ ent ] \} \{ diffusive porosity

$\{$ water gas conc [ entration ] $\}\{$ gas fracture sat [ uration ] \} 


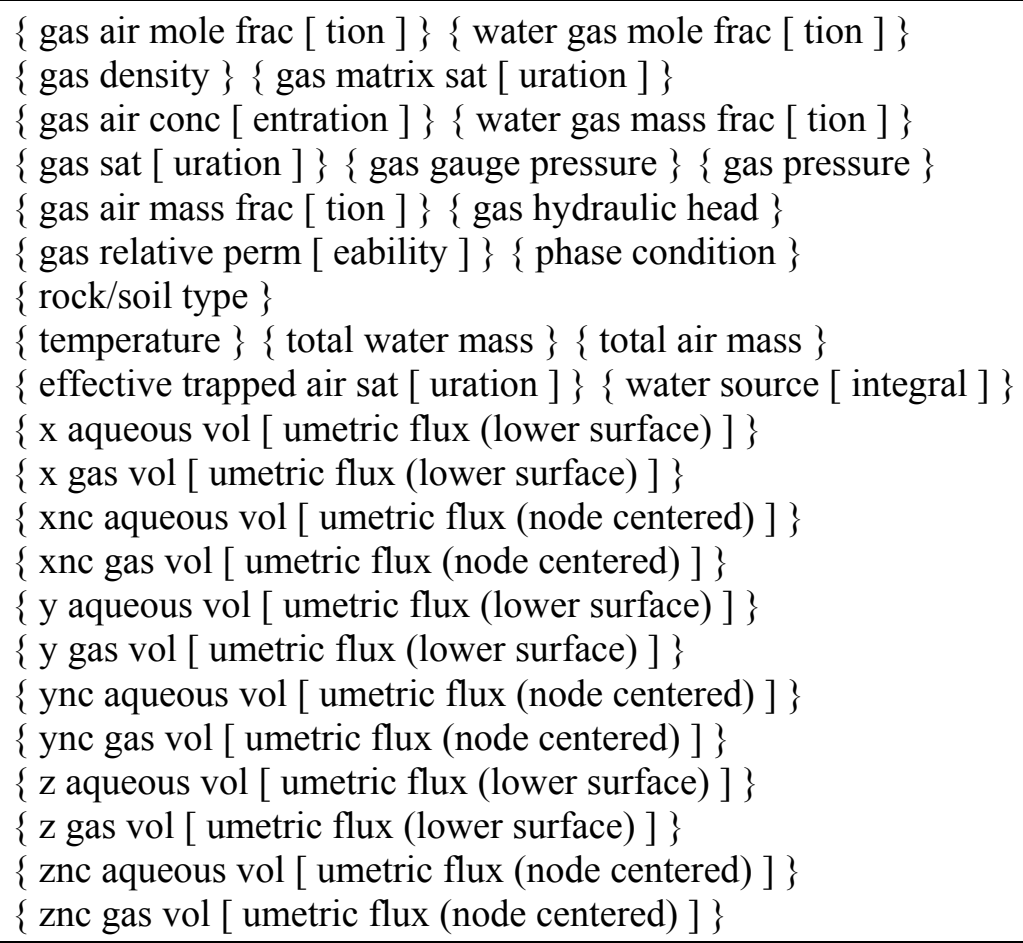

\section{A.6.3 Reference Node Variable and Plot File Variable Options}

Elseif: Operational Mode Option: Solution Control Card $=\{$ Water-Air-Energy $\}$

$\{$ air source [ integral ] $\}\{$ apparent water sat [ uration ] \}

$\{$ air aqueous mass frac [ tion ] $\}\{$ water aqueous mass frac [ tion ] \}

\{aqueous hydraulic head $\}$ \{air aqueous conc [ entration ] \}

$\{$ water aqueous conc [ entration ] \} \{ aqueous relative perm [ eability ]

$\{$ aqueous moisture cont [ ent ] \} \{ aqueous density

$\{$ aqueous fracture sat [ uration ] \} \{ aqueous gauge pressure \}

$\{$ aqueous matrix sat [ uration ] \} \{aqueous sat [ uration ] \} \{aqueous pressure \}

$\{$ diffusive porosity $\}$ \{ energy source [integral ] $\}\{$ gas matrix sat [ uration ]

$\{$ gas air mole frac [ tion ] $\}\{$ water gas conc [ entration ] $\}\{$ water gas mole frac [ tion ]

\{ gas air conc [ entration ] $\}\{$ gas fracture sat [ uration ] \} \{ gas sat [ uration ] \}

\{ gas gauge pressure $\}\{$ gas pressure $\}\{$ water gas mass frac [ tion ]

$\{$ gas air mass frac [ tion ] $\}$

$\{$ gas relative perm [ eability ] $\}$ gas density $\}$ gas hydraulic head

\{phase condition $\}$ \{rock/soil type

\{temperature $\}$ total water mass $\}$ \{total air mass \}

$\{$ effective trapped air sat [ uration ] $\}\{$ water source [ integral ] \}

$\{\mathrm{x}$ aqueous vol [ umetric flux (lower surface) ] \}

$\{\mathrm{x}$ gas vol [ umetric flux (lower surface) ] \}

$\{\mathrm{x}$ heat flux [ (lower surface) $]\}$

$\{\mathrm{x}$ thermal cond [ uctivity ] \}

$\{$ xnc aqueous vol [ umetric flux (node centered) ] \}

$\{$ xnc gas vol [ umetric flux (node centered) $]\}\{$ xnc heat flux [ (node centered) ]

$\{$ y aqueous vol [ umetric flux (lower surface) ] \}

$\{$ y gas vol [ umetric flux (lower surface) ] \}

$\{\mathrm{y}$ heat flux [ (lower surface) $]\}$ 


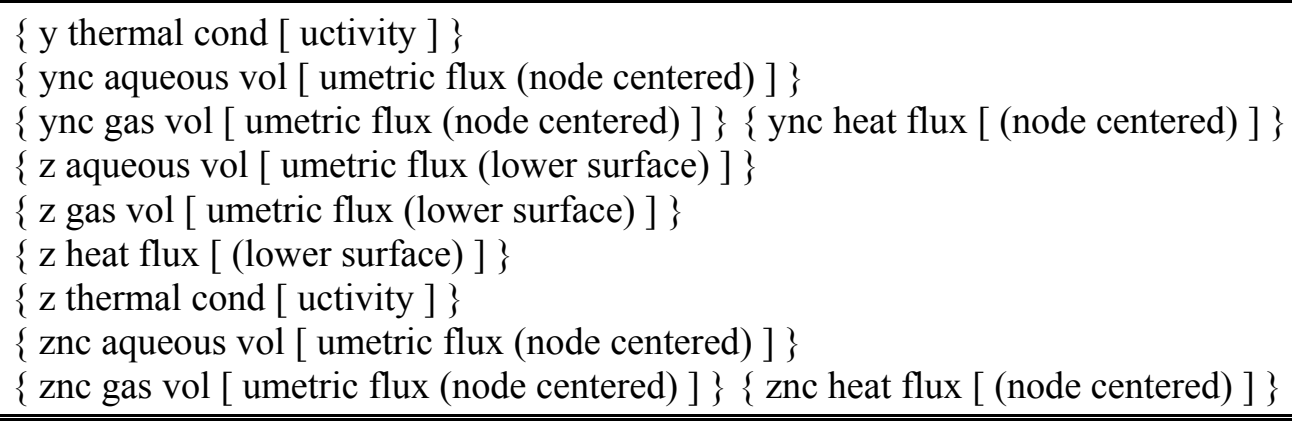

\section{A.6.4 Default Units for the Reference Node and Plot File Variables}

Air Concentration $\left(\mathrm{kg} / \mathrm{m}^{\wedge} 3\right)$

Water Concentration $\left(\mathrm{kg} / \mathrm{m}^{\wedge} 3\right)$

Aqueous Relative Permeability (null)

Aqueous Volumetric Flux (m/s)

Energy Source Integral (J)

Gas Sat [ uration ] (null)

Gauge Pressure $(\mathrm{Pa})$

Heat Flux $\left(\mathrm{W} / \mathrm{m}^{\wedge} 2\right)$

Phase Condition (null)

Relative Permeability (null)

Temperature $(\mathrm{C})$
Air Source Integral (kg)

Water Source Integral (kg)

Aqueous Sat [ uration ] (null)

Density $\left(\mathrm{kg} / \mathrm{m}^{\wedge} 3\right)$

Gas Relative Permeability (null)

Gas Volumetric Flux (m/s)

Head (m),

Moisture Content (null)

Pressure $(\mathrm{Pa})$

Sat [ uration ] (null)

Thermal Conductivity (W/m K)

\section{A.6.5 Output Control Card Example}

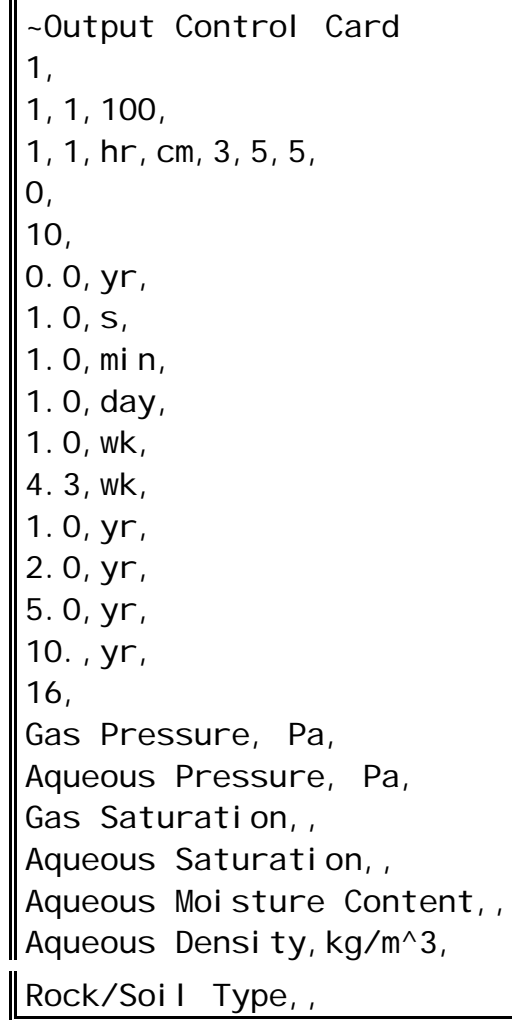




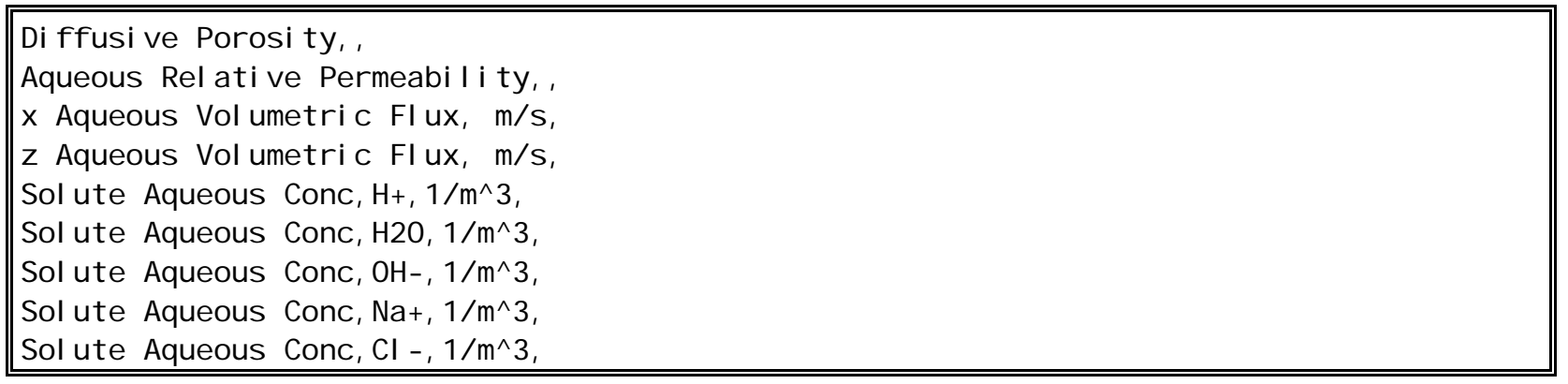

\section{A.7 Source Card}

Card Title ${ }^{\mathrm{a}}\{\sim$ Source [ Card ] $\}$

Format: Char $^{a}$

Number of Source Domains ${ }^{\mathrm{a}}$,

Format: Integer ${ }^{\mathrm{a}}$,

For: Number of Source Domains

If: Operational Mode Option: Solution Control Card $=\{$ Water $\}$

Source Type Option ${ }^{\mathrm{a}}$

\{Aqueous Volumetric $\}$ Aqueous Mass $\}$ W Well \} I-Start Index ${ }^{\mathrm{b}}$, I-End Index ${ }^{\mathrm{c}}$, J-Start Index ${ }^{\mathrm{d}}$, J-End Index ${ }^{\mathrm{e}}$, K-Start Index ${ }^{\mathrm{f}}$, K-End Index ${ }^{\mathrm{g}}$, Number of Source Times ${ }^{\mathrm{h}}$, Format: Char $^{a}$, Integer ${ }^{b}$, Integer $^{c}$, Integer ${ }^{d}$, Integer $^{e}$, Integer $^{f}$, Integer $^{g}$, Integer $^{h}$,

Elseif: Operational Mode Option: Solution Control Card $=\{$ Water-Air $\}$

Source Type Option ${ }^{\mathrm{a}}$

\{Aqueous Volumetric \} A Aqueous Mass \} Gas Volumetric w/ Mass Fraction

\{Gas Volumetric w/ Relative Humidity \} Gas Mass w/ Mass Fraction \}

\{ Gas Mass w/ Relative Humidity \}

I-Start Index ${ }^{\mathrm{b}}$, I-End Index ${ }^{\mathrm{c}}$, J-Start Index ${ }^{\mathrm{d}}$, J-End Index ${ }^{\mathrm{e}}$,

K-Start Index ${ }^{\mathrm{f}}, \mathrm{K}$-End Index ${ }^{\mathrm{g}}$, Number of Source Times ${ }^{\mathrm{h}}$,

Format: Char $^{a}$, Integer $^{b}$, Integer $^{c}$, Integer $^{d}$, Integer $^{e}$, Integer $^{f}$, Integer $^{g}$, Integer $^{h}$,

Elseif: Operational Mode Option: Solution Control Card = $\{$ Water-Air-Energy $\}$

Source Type Option ${ }^{\mathrm{a}}$

\{Power \{ Power Density $\}$ Aqueous Volumetric \} Aqueous Mass

\{Gas Volumetric w/ Mass Fraction \} \{ Gas Volumetric w/ Relative Humidity

\{ Gas Mass w/ Mass Fraction \} \{ Gas Mass w/ Relative Humidity \}

I-Start Index ${ }^{\mathrm{b}}$, I-End Index ${ }^{\mathrm{c}}$, J-Start Index ${ }^{\mathrm{d}}$, J-End Index ${ }^{\mathrm{e}}$,

K-Start Index ${ }^{\mathrm{f}}, \mathrm{K}-$ End Index ${ }^{\mathrm{g}}$, Number of Source Times ${ }^{\mathrm{h}}$,

Format: Char $^{a}$, Integer $^{b}$, Integer $^{c}$, Integer $^{d}$, Integer $^{e}$, Integer $^{f}$, Integer $^{g}$, Integer $^{h}$,

If: Operational Mode Option: Solution Control Card $=\{$ Water $\}$

For: Number of Source Times

Source Time ${ }^{\mathrm{a}}$, Units $^{\mathrm{b}}(\mathrm{s})$, 


\begin{tabular}{|c|}
\hline 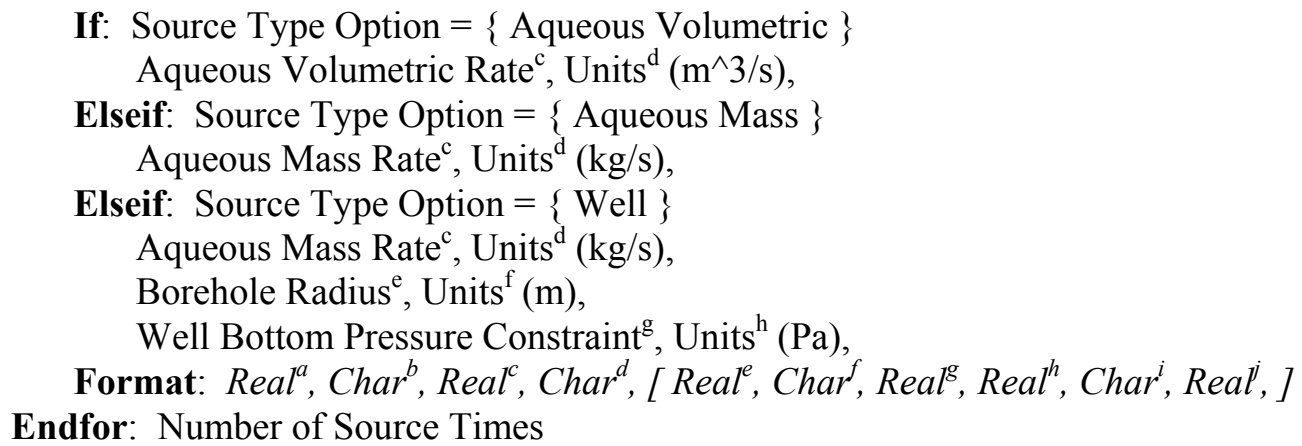 \\
\hline
\end{tabular}

Elseif: Operational Mode Option: Solution Control Card $=\{$ Water-Air $\}$

For: Number of Source Times

Source Time ${ }^{\mathrm{a}}$, Units ${ }^{\mathrm{b}}(\mathrm{s})$,

If: Source Type Option $=\{$ Aqueous Volumetric $\}$

Pressure $^{\mathrm{c}}$, Units $^{\mathrm{d}}(\mathrm{Pa})$, Aqueous Volumetric Rate ${ }^{\mathrm{e}}, \operatorname{Units}^{\mathrm{f}}\left(\mathrm{m}^{\wedge} 3 / \mathrm{s}\right)$,

Dissolved Air Mass Fraction ${ }^{\mathrm{g}}$

Elseif: Source Type Option $=\{$ Aqueous Mass $\}$

Pressure $^{\mathrm{c}}$, Units ${ }^{\mathrm{d}}(\mathrm{Pa})$, Aqueous Mass Rate ${ }^{\mathrm{e}}, \operatorname{Units}^{\mathrm{f}}(\mathrm{kg} / \mathrm{s})$,

Dissolved Air Mass Fraction ${ }^{\mathrm{g}}$

Elseif: Source Type Option $=\{$ Gas Mass w/ Mass Fraction $\}$

Pressure $^{\mathrm{c}}$, Units ${ }^{\mathrm{d}}(\mathrm{Pa})$, Gas Mass Rate ${ }^{\mathrm{e}}, \operatorname{Units}^{\mathrm{f}}(\mathrm{kg} / \mathrm{s})$,

Water Vapor Mass Fraction ${ }^{\mathrm{g}}$

Elseif: Source Type Option $=\{$ Gas Mass w/ Relative Humidity $\}$

Pressure $^{\mathrm{c}}, \operatorname{Units}^{\mathrm{d}}(\mathrm{Pa})$, Gas Mass Rate ${ }^{\mathrm{e}}$, Units $^{\mathrm{f}}(\mathrm{kg} / \mathrm{s})$,

Water Vapor Relative Humidity ${ }^{\mathrm{g}}$

Elseif: Source Type Option $=\{$ Gas Volumetric w/ Mass Fraction $\}$

Pressure $^{\mathrm{c}}$, Units ${ }^{\mathrm{d}}(\mathrm{Pa})$, Gas Volumetric Rate ${ }^{\mathrm{e}}$, Units ${ }^{\mathrm{f}}\left(\mathrm{m}^{\wedge} 3 / \mathrm{s}\right)$,

Water Vapor Mass Fraction ${ }^{\mathrm{g}}$

Elseif: Source Type Option $=\{$ Gas Volumetric w/ Relative Humidity $\}$

Pressure $^{\mathrm{c}}$, Units ${ }^{\mathrm{d}}(\mathrm{Pa})$, Gas Volumetric Rate ${ }^{\mathrm{e}}$, Units ${ }^{\mathrm{f}}\left(\mathrm{m}^{\wedge} 3 / \mathrm{s}\right)$,

Water Vapor Relative Humidity ${ }^{\mathrm{g}}$

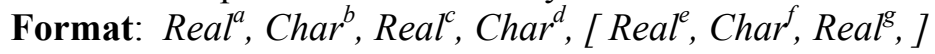

Endfor: Number of Source Times

Elseif: Operational Mode Option: Solution Control Card $=\{$ Water-Air-Energy $\}$

For: Number of Source Times

Source Time ${ }^{\mathrm{a}}$, Units ${ }^{\mathrm{b}}$ (s),

If: Source Type Option $=\{$ Power $\}$

Power ${ }^{\mathrm{c}}$, Units ${ }^{\mathrm{d}}(\mathrm{W})$

Elseif: Source Type Option $=\{$ Power Density $\}$

Power Density ${ }^{\mathrm{c}}$, Units ${ }^{\mathrm{d}}\left(\mathrm{W} / \mathrm{m}^{\wedge} 3\right)$

Elseif: Source Type Option $=\{$ Aqueous Volumetric $\}$

Temperature $^{\mathrm{c}}, \operatorname{Units}^{\mathrm{d}}(\mathrm{C})$, Pressure $^{\mathrm{e}}, \operatorname{Units}^{\mathrm{f}}(\mathrm{Pa})$,

Aqueous Volumetric Rate ${ }^{\mathrm{g}}$, Units ${ }^{\mathrm{h}}\left(\mathrm{m}^{\wedge} 3 / \mathrm{s}\right)$, Dissolved Air Mass Fraction ${ }^{\mathrm{i}}$

Elseif: Source Type Option $=\{$ Aqueous Mass $\}$

Temperature $^{\mathrm{c}}, \operatorname{Units}^{\mathrm{d}}(\mathrm{C})$, Pressure $^{\mathrm{e}}, \operatorname{Units}^{\mathrm{f}}(\mathrm{Pa})$,

Aqueous Mass Rate ${ }^{\mathrm{g}}$, Units ${ }^{\mathrm{h}}(\mathrm{kg} / \mathrm{s})$, Dissolved Air Mass Fraction ${ }^{\mathrm{i}}$ 


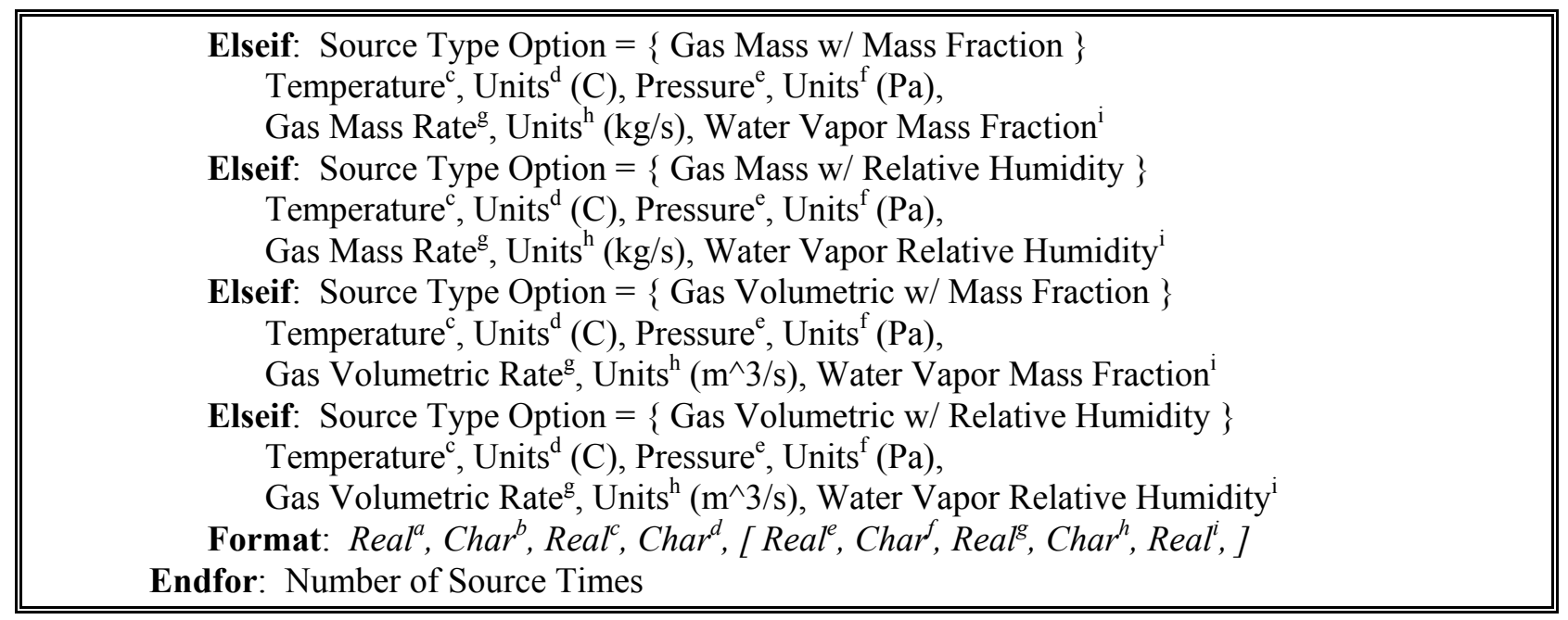

\section{A.7.1 Source Card Examples}

$\sim$ Source Card

1 ,

Aqueous Volumetric, 2, 2, 1, 1, 71, 71,6,

0 , mi n, 0.319307, gal/mi n,

$37.4675, \mathrm{mi} \mathrm{n}, 0.319307, \mathrm{gal} / \mathrm{min}$,

$74.9232, \mathrm{~min}, 0.30205, \mathrm{gal} / \mathrm{min}$,

$112.379, \mathrm{mi} \mathrm{n}, 0.303402, \mathrm{gal} / \mathrm{min}$,

$149.835, \mathrm{~min}, 0.307011, \mathrm{gal} / \mathrm{min}$,

187.29 , mi n, 0.311148, gal/mi n,

source Card

1 ,

WelI $1,1,1,1,1,20,1$,

$0, s,-0,5, f^{\wedge} 3 / s, 4,0, i n, 101325,0, P a$,

\section{A.8 Saturation Function Card}

Card Title $^{\mathrm{a}}\{\sim$ Saturation Function [ Card ] $\}$

Format: Char $^{a}$

For: Number of Rock/Soil Types

If: Operational Mode Option: Solution Control Card $=\{$ Water $\}\{$ Water-Air $\}$ or $\{$ Water-AirEnergy

Rock/Soil Name,

Saturation Function ${ }^{\mathrm{b}}$,

\{Nonhysteretic van Genuchten \} Nonhysteretic Brooks and Corey

\{ Hysteretic van Genuchten $\}$ \{ Hysteretic Brooks and Corey

\{ Haverkamp $\}$ T Tabular [ Water Content ] \}

If: Saturation Function Option $=\{$ Nonhysteretic van Genuchten $\}$

If: Rock/Soil Name $=\{\{$ Fractured $\}\}$ 


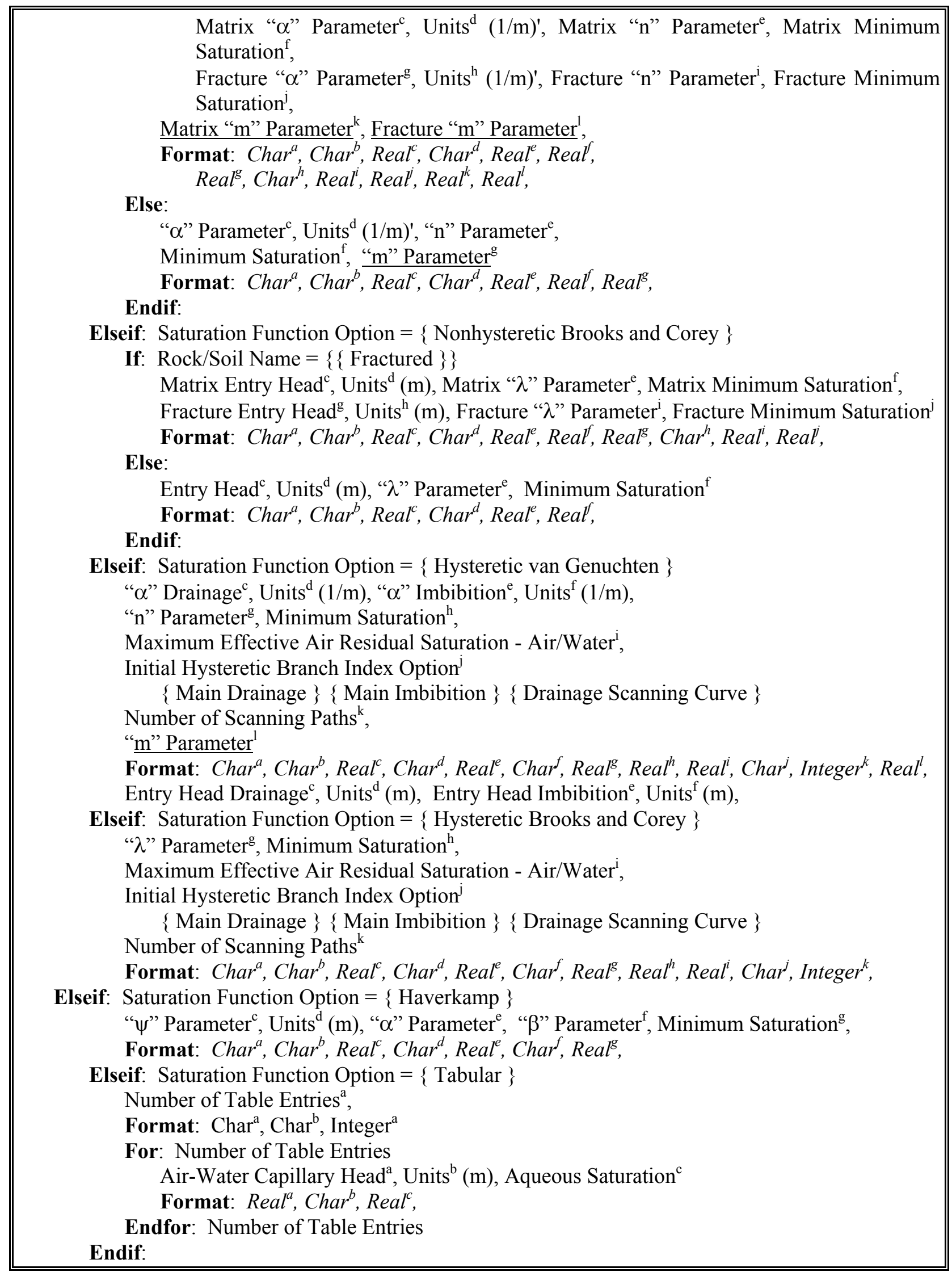




\section{A.8.1 Saturation Function Card Examples}

Saturation Function Card

Sand1, Nonhysteretic Van Genuchten, $0.0283,1 / \mathrm{cm}, 7.6,0.32,1.0,1.8,2.25$,

Saturation Function Card

Sand, Nonhysteretic Van Genuchten, $1.563,1 / \mathrm{m}, 5.4,0.15$, ,

Saturation Function Card

Geologic Media, Nonhysteretic van Genuchten, $0.2,1 / \mathrm{cm}, 1.8,0.0$, ,

Saturation Function Card

72.0, dynes/cm, 47.8, dynes/cm, ,

$20 / 30$ Ot awa Sand, Entrapment

1. 0 ,

S Saturation Function Card

SP1, Nonhysteretic Brooks and Corey, 4.32, cm, 0.528,

SP2, Nonhysteretic Brooks and Corey, 6.41, cm, 0.415,

SM-ML1, Nonhystereti C Brooks and Corey, 10.0, cm, 0.383,,

SM-SP1, Nonhysteretic Brooks and Corey, 9.0, cm, 0.430,

SP3, Nonhysteretic Brooks and Corey, 10.7, cm, 0.494,

SW1, Nonhysteretic Brooks and Corey, 1.17, cm, 0.346,

\section{A.9 Initial Conditions Card}

Card Title $^{\mathrm{a}}\{\sim$ Initial [ Conditions Card ] $\}$

Format: Char $^{a}$

If: Operational Mode Option: Solution Control Card $=\{$ Water $\}\{$ Water-Air $\}$

\{ Water-Air-Energy

Initial Saturation Option ${ }^{\mathrm{a}}$, Initial Saturation Option ${ }^{\mathrm{b}}$,

\{ Gas Pressure, Aqueous Pressure \}

\{ Gas Pressure, Aqueous Saturation \}

\{Aqueous Pressure, Aqueous Saturation \}

Format: Char $^{a}$, Char $^{b}$,

Number of Initial Conditions Domains ${ }^{\mathrm{a}}$

Format: Integer ${ }^{a}$,

For: Number of Initial Conditions Domains

If: Operational Mode Option: Solution Control Card $=\{$ Water $\}$

Variable Name Option ${ }^{\mathrm{a}}$

\{Aqueous Pressure \} \{ Gas Pressure \} \{ Temperature \} Aqueous Saturation

Elseif: Operational Mode Option: Solution Control Card $=\{$ Water-Air $\}\{$ Water-Air-Energy $\}$

Variable Name Option ${ }^{\mathrm{a}}$

\{Aqueous Pressure $\}$ \{ Gas Pressure $\}$ \{ Temperature $\}$

\{Aqueous Saturation \} \{ Air Partial Pressure \} A Air Gas Mole Fraction

\{Air Aqueous Mole Fraction $\}$ \{ Trapped Air Saturation \} 


\begin{tabular}{|c|}
\hline 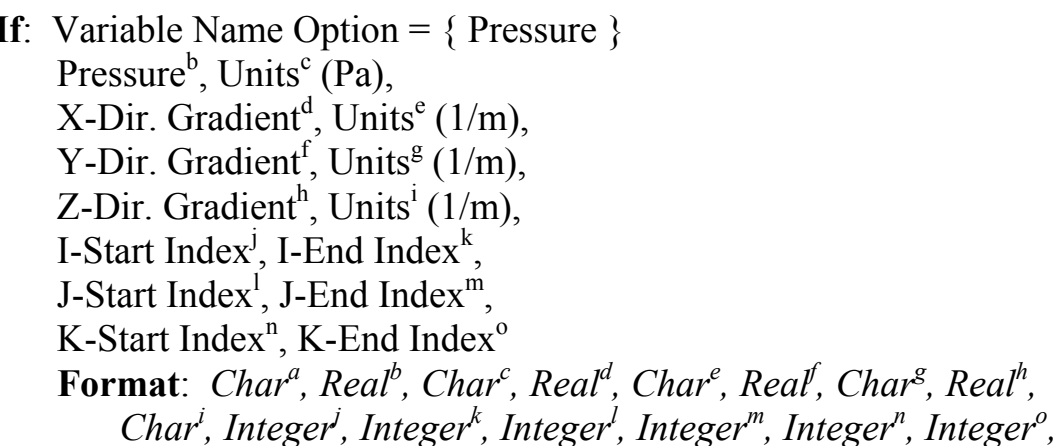 \\
\hline
\end{tabular}

Elseif: Variable Name Option $=\{$ Temperature $\}$

Temperature $^{\mathrm{b}}$, Units ${ }^{\mathrm{c}}(\mathrm{C})$,

X-Dir. Gradient ${ }^{\mathrm{d}}$, Units ${ }^{\mathrm{e}}(1 / \mathrm{m})$,

Y-Dir. Gradient ${ }^{\mathrm{f}}$, Units ${ }^{\mathrm{g}}(1 / \mathrm{m})$,

Z-Dir. Gradient ${ }^{\mathrm{h}}$, Units ${ }^{\mathrm{i}}(1 / \mathrm{m})$,

I-Start Index ${ }^{\mathrm{j}}$, I-End Index ${ }^{\mathrm{k}}$,

J-Start Index ${ }^{1}$, J-End Index ${ }^{\mathrm{m}}$,

K-Start Index ${ }^{\mathrm{n}}$, K-End Index ${ }^{\mathrm{o}}$

Format: Char $^{a}$, Real $^{b}$, Char $^{c}$, Real $^{d}$, Char $^{e}$, Reall $^{\prime}$, Char $^{g}$, Real $^{h}$,

Char $^{i}$, Integer $^{j}$, Integer $^{k}$, Integer ${ }^{l}$, Integer ${ }^{m}$, Integer $^{n}$, Integer $^{o}$,

Elseif: Variable Name Option $=\{$ Saturation $\}$

Saturation $^{\mathrm{b}}$, Null ${ }^{\mathrm{c}}$,

X-Dir. Gradient ${ }^{\mathrm{d}}$, Units ${ }^{\mathrm{e}}(1 / \mathrm{m})$,

Y-Dir. Gradient ${ }^{\mathrm{f}}$, Units $^{\mathrm{g}}(1 / \mathrm{m})$,

Z-Dir. Gradient ${ }^{\mathrm{h}}$, Units ${ }^{\mathrm{i}}(1 / \mathrm{m})$,

I-Start Index ${ }^{\mathrm{j}}$, I-End Index ${ }^{\mathrm{k}}$,

J-Start Index ${ }^{1}$, J-End Index ${ }^{\mathrm{m}}$,

K-Start Index ${ }^{\mathrm{n}}$, K-End Index ${ }^{\mathrm{o}}$

Format: Char ${ }^{a}$, Real $^{b}$, Null $^{c}$, Real $^{d}$, Char $^{e}$, Real $^{f}$, Char $^{g}$, Real $^{h}$, Char $^{i}$, Integer $^{j}$, Integer $^{k}$, Integer $^{l}$, Integer $^{m}$, Integer ${ }^{n}$, Integer ${ }^{o}$,

Elseif: Variable Name Option $=\{$ Mass Fraction $\}$

Mole Fraction ${ }^{\mathrm{b}}, \mathrm{Null}^{\mathrm{c}}$,

X-Dir. Gradient ${ }^{\mathrm{d}}$, Units ${ }^{\mathrm{e}}(1 / \mathrm{m})$,

Y-Dir. Gradient ${ }^{\mathrm{f}}$, Units ${ }^{\mathrm{g}}(1 / \mathrm{m})$,

Z-Dir. Gradient ${ }^{\mathrm{h}}$, Units ${ }^{\mathrm{i}}(1 / \mathrm{m})$,

I-Start Index ${ }^{\mathrm{j}}$, I-End Index ${ }^{\mathrm{k}}$,

J-Start Index ${ }^{1}$, J-End Index ${ }^{\mathrm{m}}$,

K-Start Index ${ }^{\mathrm{n}}$, K-End Index ${ }^{\mathrm{o}}$

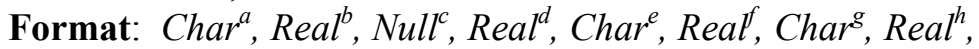

Char $^{i}$, Integer ${ }^{j}$, Integer ${ }^{k}$, Integer ${ }^{l}$, Integer ${ }^{m}$, Integer ${ }^{n}$, Integer ${ }^{o}$,

Elseif: Variable Name Option $=\{$ Mole Fraction $\}$

Mole Fraction ${ }^{\mathrm{b}}$,Null ${ }^{\mathrm{c}}$,

X-Dir. Gradient ${ }^{\mathrm{d}}$, Units ${ }^{\mathrm{e}}(1 / \mathrm{m})$,

Y-Dir. Gradient ${ }^{\mathrm{f}}$, Units ${ }^{\mathrm{g}}(1 / \mathrm{m})$, 
Z-Dir. Gradient ${ }^{\mathrm{h}}$ Units $^{\mathrm{i}}(1 / \mathrm{m})$,

I-Start Index ${ }^{\mathrm{j}}$, I-End Index ${ }^{\mathrm{k}}$,

J-Start Index ${ }^{1}$, J-End Index ${ }^{\mathrm{m}}$,

$\mathrm{K}$-Start Index ${ }^{\mathrm{n}}, \mathrm{K}$-End Index ${ }^{\mathrm{o}}$

Format: Char ${ }^{a}$, Real $^{b}$, Null $^{c}$, Real $^{d}$, Char $^{e}$, Realf $^{\prime}$ Char $^{g}$, Real $^{h}$, Char $^{i}$, Integer $^{j}$, Integer $^{k}$, Integer $^{l}$, Integer $^{m}$, Integer $^{n}$, Integer $^{o}$,

Elseif: Variable Name Option $=\{$ Relative Humidity $\}$

Relative Humidity ${ }^{\mathrm{b}} \mathrm{Null}^{\mathrm{c}}$,

X-Dir. Gradient ${ }^{\mathrm{d}}$, Units ${ }^{\mathrm{e}}(1 / \mathrm{m})$,

Y-Dir. Gradient ${ }^{\mathrm{f}}$, Units $^{\mathrm{g}}(1 / \mathrm{m})$,

Z-Dir. Gradient ${ }^{\mathrm{h}}$, Units ${ }^{\mathrm{i}}(1 / \mathrm{m})$,

I-Start Index ${ }^{\mathrm{j}}$, I-End Index ${ }^{\mathrm{k}}$,

J-Start Index ${ }^{1}$, J-End Index ${ }^{\mathrm{m}}$,

K-Start Index ${ }^{\mathrm{n}}$, K-End Index ${ }^{\mathrm{o}}$

Format: Char ${ }^{a}$, Real $^{b}$, Null $^{c}$, Real ${ }^{d}$, Char ${ }^{e}$, Real $^{\prime}$, Char $^{g}$, Real ${ }^{h}$,

Char $^{i}$, Integer $^{j}$, Integer $^{k}$, Integer ${ }^{l}$, Integer ${ }^{m}$, Integer $^{n}$, Integer $^{o}$,

Endfor: Number of Initial Conditions Domains

Endcard: Initial Conditions Card

\section{A.9.1 Initial Conditions Card Examples}

I nitial Conditions Card

Aqueous Pressure, Gas Pressure,

4 ,

Temper at ure, 20, C, 0,1/ $\mathrm{m}, 0,1 / \mathrm{m}, 0,1 / \mathrm{m}, 1,52,1,1,1,113$,

Aqueous Pressure, $183254, \mathrm{~Pa}, 0,1 / \mathrm{m}, 0,1 / \mathrm{m},-9793.52,1 / \mathrm{m}, 1,52,1,1,1,113$,

Aqueous Pressure, 92956, Pa, 0,1/ m, 0,1/ $\mathrm{m}, 0,1 / \mathrm{m}, 2,2,1,1,71,100$,

Gas Pressure, $92956, \mathrm{~Pa}, 0,1 / \mathrm{m}, 0,1 / \mathrm{m}, 0,1 / \mathrm{m}, 1,52,1,1,1,113$,

I nitial Conditions Card

Gas Pressure, Aqueous Pressure,

1 ,

Aqueous Pressure, 121325., Pa, , , , - $9793.5331,1 / \mathrm{m}, 1,20,1,1,1,10$,

unitial Conditions Card

Aqueous Saturation, Gas Pressure,

3 ,

Aqueous Saturation, 0.5, , , , , , 1, 50, 1, 1, 1, 1,

Gas Pressure, $101330, \mathrm{~Pa}, 1,1,1,1,50,1,1,1,1$,

Temper at ure, $70.0, C_{1}, \ldots, 1,1,50,1,1,1,1$, 


\section{A.10 Mechanical Properties Card}

Card Title $^{\mathrm{a}}\{\sim$ Mechanical [ Properties Card ] $\}$

Format: Char $^{a}$

For: Number of Rock/Soil Types

If: Operational Mode Option: Solution Control Card $=\{$ Water $\}$

Rock/Soil Name ${ }^{a}$,

Particle Density $^{\mathrm{b}}(2650.0), \underline{\text { Units }}^{\mathrm{c}}\left(\mathrm{kg} / \mathrm{m}^{\wedge} 3\right)$,

If: Rock/Soil Name $=\{\{$ Fractured $\}\}$

Matrix Total Porosity ${ }^{\mathrm{d}}$, Matrix Diffusive Porosity ${ }^{\mathrm{e}}$,

Fracture Total Porosity ${ }^{\mathrm{f}}$, Fracture Diffusive Porosity ${ }^{\mathrm{g}}$,

Specific Storativity ${ }^{\mathrm{h}}$, Units $^{\mathrm{i}}(1 / \mathrm{m})$,

Format: Char $^{a}$, Real $^{b}$, Char $^{c}$, Real $^{d}$, Real ${ }^{e}$, Real $^{\prime}$, Real $^{g}$, Real $^{h}$, Char $^{i}$,

Else:

Total Porosity ${ }^{\mathrm{d}}$, Diffusive Porosity ${ }^{\mathrm{e}}$,

Specific Storativity ${ }^{\mathrm{f}}$ Units $^{\mathrm{g}}(1 / \mathrm{m})$,

Format: Char $^{a}$, Real $^{b}$, Char $^{c}$, Real $^{d}$, Real, Real, Char $^{g}$,

Endif:

Elseif: Operational Mode Option: Solution Control Card $=\{$ Water-Air $\}\{$ Water-Air-Energy $\}$

Rock/Soil Name ${ }^{a}$,

Particle Density $^{\mathrm{b}}(2650.0), \underline{\text { Units }}^{\mathrm{c}}\left(\mathrm{kg} / \mathrm{m}^{\wedge} 3\right)$,

If: Rock/Soil Name $=\{\{$ Fractured $\}\}$

Matrix Total Porosity ${ }^{\mathrm{d}}$, Matrix Diffusive Porosity ${ }^{\mathrm{e}}$,

Fracture Total Porosity ${ }^{\mathrm{f}}$, Fracture Diffusive Porosity ${ }^{\mathrm{g}}$,

$\underline{\text { Specific Storativity }}^{\mathrm{h}}$, Units $^{\mathrm{i}}(1 / \mathrm{m})$,

Tortuosity Function Option ${ }^{\mathrm{j}}$

\{Constant $\}$ Millington and Quirk \}

If: Tortuosity Function Option $=\{$ Constant $\}$

Aqueous-Phase Tortuosity ${ }^{\mathrm{k}}$, Gas-Phase Tortuosity ${ }^{1}$,

Format: Char $^{a}$, Real $^{b}$, Char $^{c}$, Real $^{d}$, Real $^{e}$, Real ,

Real $^{g}$, Real $^{h}$, Char $^{i}$, Char $^{j}$, Real $^{k}$, Real $^{\prime}$,

Else:

Format: Char $^{a}$, Real $^{b}$, Char $^{c}$, Real $^{d}$, Real $^{e}$, Real , Real $^{g}$, Real $^{h}$, Char $^{i}, \mathrm{Char}^{j}$,

Endif:

Else:

Total Porosity ${ }^{\mathrm{d}}$, Diffusive Porosity ${ }^{\mathrm{e}}$,

$\underline{\text { Specific Storativity }}^{\mathrm{f}}{\underline{\text { Units }^{\mathrm{g}}}}^{\mathrm{g}}(1 / \mathrm{m})$,

Tortuosity Function Option ${ }^{\mathrm{h}}$

\{Constant $\}$ \{ Millington and Quirk

If: Tortuosity Function Option $=\{$ Constant $\}$

Aqueous-Phase Tortuosity, Gas-Phase Tortuosity ${ }^{\mathrm{j}}$,

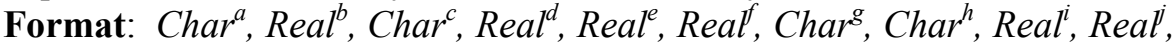

Else:

Format: Char $^{a}$, Real $^{b}$, Char $^{c}$, Real $^{d}$, Real $^{e}$, Real $^{f}$, Char $^{g}$, Char $^{h}$,

\section{Endif:}

Endif: 
Endfor: Number of Rock/Soil Types

Endcard: Mechanical Properties Card

\section{A.10.1 Mechanical Properties Card Examples}

Mechanical Properties Card

$S P 1,2.63, \mathrm{~g} / \mathrm{cm}^{\wedge} 3,0.19,0.19,1$, ,

SP2, 2.63, $\mathrm{g} / \mathrm{cm}^{\wedge} 3,0.24,0.24, \ldots$,

SM-ML 1, 2.63, g/ cm^3,0.35,0.35, , ,

SM- SP $1,2.63, \mathrm{~g} / \mathrm{cm}^{\wedge} 3,0.37,0.37, \ldots$

SP 3, 2.63, g/ c $\mathrm{m}^{\wedge} 3,0.27,0.27,1$,

SW1, 2.63, $\mathrm{g} / \mathrm{cm}^{\wedge} 3,0.28,0.28, \ldots$,

US, $2.63, \mathrm{~g} / \mathrm{cm}^{\wedge} 3,0.96,0.96, \ldots$,

Mechanical Properties Card

Sand $1,2650, \mathrm{~kg} / \mathrm{m}^{\wedge} 3,0.34,0.34,0$, Mill ington and Quirk, ,

Mechanical Properties Card

Sand, $2650, \mathrm{~kg} / \mathrm{m}^{\wedge} 3,0.4,0.4$, , Const ant, 0.5,0.5,

Mechanical Properties Card

Geologic Media, , 0.35,0.35, , Constant Diffusion, 1.0,

Mechanical Properties Card

$20 / 30$ Ot tawa Sand, $2650, \mathrm{~kg} / \mathrm{m}^{\wedge} 3,0.3431,0.3431,0,1 / \mathrm{m}$, Millington and Quirk,

\section{A.11 Hydraulic Properties Card}

Card Title $^{\mathrm{a}}\{\sim$ Hydraulic [ Properties Card ] $\}$

Format: Char $^{a}$

For: Number of Rock/Soil Types

Rock/Soil Namea,

If: Rock/Soil Name $=\{\{$ Fractured $\}\}$

X-Dir. Matrix Intrinsic Perm. or Hydraulic Cond. ${ }^{b}$, Units $^{c}\left(\mathrm{~m}^{\wedge} 2\right.$ or hc $\left.\mathrm{m} / \mathrm{s}\right)$,

Y-Dir. Matrix Intrinsic Perm. or Hydraulic Cond. ${ }^{d}$, Units $^{\mathrm{e}}\left(\mathrm{m}^{\wedge} 2\right.$ or hc $\left.\mathrm{m} / \mathrm{s}\right)$,

Z-Dir. Matrix Intrinsic Perm. or Hydraulic Cond. ${ }^{\mathrm{f}}$, Units $^{\mathrm{g}}\left(\mathrm{m}^{\wedge} 2\right.$ or hc $\left.\mathrm{m} / \mathrm{s}\right)$,

X-Dir. Fracture Intrinsic Perm. or Hydraulic Cond. ${ }^{\mathrm{h}}$, Units ${ }^{\mathrm{i}}\left(\mathrm{m}^{\wedge} 2\right.$ or hc $\left.\mathrm{m} / \mathrm{s}\right)$,

Y-Dir. Fracture Intrinsic Perm. or Hydraulic Cond. ${ }^{j}$, Units ${ }^{\mathrm{k}}\left(\mathrm{m}^{\wedge} 2\right.$ or hc $\left.\mathrm{m} / \mathrm{s}\right)$,

Z-Dir. Fracture Intrinsic Perm. or Hydraulic Cond. ${ }^{1}$ Units ${ }^{\mathrm{m}}\left(\mathrm{m}^{\wedge} 2\right.$ or hc $\left.\mathrm{m} / \mathrm{s}\right)$,

Intrinsic Permeability Function ${ }^{n}$,

\{Constant \} \{Kozeny and Carmen \} \{ Fair and Hatch \}

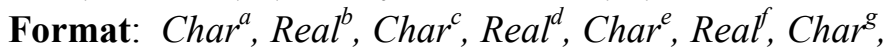

Real $^{h}$, Char $^{i}$, Real ${ }^{j}$, Char $^{k}$, Real $^{l}$, Char $^{m}$, Char $^{n}$,

Else:

X-Dir. Intrinsic Permeability or Hydraulic Conductivity ${ }^{b}$, Units $^{c}\left(\mathrm{~m}^{\wedge} 2\right.$ or hc $\left.\mathrm{m} / \mathrm{s}\right)$,

Y-Dir. Intrinsic Permeability or Hydraulic Conductivity ${ }^{\mathrm{d}}$, Units ${ }^{\mathrm{e}}\left(\mathrm{m}^{\wedge} 2\right.$ or hc $\left.\mathrm{m} / \mathrm{s}\right)$,

Z-Dir. Intrinsic Permeability or Hydraulic Conductivity ${ }^{\mathrm{f}}, \mathrm{Units}^{\mathrm{g}}\left(\mathrm{m}^{\wedge} 2\right.$ or hc $\left.\mathrm{m} / \mathrm{s}\right)$, 


Intrinsic Permeability Function ${ }^{h}$,
$\quad$ Constant $\}\{$ Kozeny and Carmen $\}\{\text { Fair and Hatch }\}^{\text {Format: } \text { Char }^{a}, \text { Real }^{b}, \text { Char }^{c}, \text { Real }^{d}, \text { Char }^{e}, \text { Realf }^{\prime}, \text { Char }^{g}, \text { Char }^{h},}$
Endif:
Endfor: Number of Rock/Soil Types

Endcard: Hydraulic Properties Card

\section{A.11.1 Hydraulic Properties Card Examples}

Hydraulic Properties Card

$20 / 30$ Ot t awa Sand, $1.6 \mathrm{e}-7, \mathrm{~cm}^{\wedge} 2,1.6 \mathrm{e}-7, \mathrm{~cm}^{\wedge} 2,1.6 \mathrm{e}-7, \mathrm{~cm}^{\wedge} 2, \mathrm{Fair}$ and Hat $\mathrm{ch}$,

นHydralic Properties Card

Geologic Media, 1.020408e-9, m^2, , 1.020408e-9, m^2, Kozeny and Carmen,

Hydraulic Properties Card

Sand $1,1,1,7,5, h c \mathrm{~m} / \mathrm{day}$, Constant,

$\sim$ Hydraulic Properties Card

SP1,4,1987e-12, $\mathrm{m}^{\wedge} 2,, 4,1987 \mathrm{e}-12, \mathrm{~m}^{\wedge} 2$, Constant,

SP2, 9. $3436 \mathrm{e}-13, \mathrm{~m}^{\wedge} 2,,, 9.3436 \mathrm{e}-13, \mathrm{~m}^{\wedge} 2$, Constant,

SM-ML1, 5. $3223 \mathrm{e}-13, \mathrm{~m}^{\wedge} 2,,, 5.3223 \mathrm{e}-13, \mathrm{~m}^{\wedge} 2$, Constant,

SM-SP1, 7.695e-12, $\mathrm{m}^{\wedge} 2,, 1,5.13 \mathrm{e}-12, \mathrm{~m}^{\wedge} 2$, Constant,

SP3, 5. $505 \mathrm{e}-12, \mathrm{~m}^{\wedge} 2,,, 3.67 \mathrm{e}-12, \mathrm{~m}^{\wedge} 2$, Constant,

SW1, 9.195e-12, $\mathrm{m}^{\wedge} 2,1,6.13 \mathrm{e}-12, \mathrm{~m}^{\wedge} 2$, Constant,

US, 1.0e+04, hc c m/ hr, , 1.0e+06, hc c m/ hr, Constant,

Hydraulic Properties Card

Sand, 5.0e-4, hc ft/s, 5.0e-4, hc ft/s, 5.0e-4, hc ft/s, constant,

\section{A.12 Thermal Properties Card}

Card Title $^{\mathrm{a}}\{\sim$ Thermal [ Properties Card ] $\}$

Format: Char $^{a}$

For: Number of Rock/Soil Types

Rock/Soil Name ${ }^{\mathrm{a}}$,

Thermal Conductivity Function Option ${ }^{\mathrm{b}}$,

\{Constant $\}\{$ Parallel $\}\{$ Linear $\}$ Somerton $\}$ C Campbell $\}$

If: Thermal Conductivity Function Option $=\{$ Constant $\}$

X-Dir. Thermal Conductivity ${ }^{\mathrm{c}}$, Units ${ }^{\mathrm{d}}(\mathrm{W} / \mathrm{m} \mathrm{K})$,

Y-Dir. Thermal Conductivity ${ }^{\mathrm{e}}, \operatorname{Units}^{\mathrm{f}}(\mathrm{W} / \mathrm{m} \mathrm{K})$,

Z-Dir. Thermal Conductivity ${ }^{\mathrm{g}}$, Units ${ }^{\mathrm{h}}(\mathrm{W} / \mathrm{m} \mathrm{K})$,

Specific Heat ${ }^{\mathrm{i}}$, Units ${ }^{\mathrm{j}}(\mathrm{J} / \mathrm{kg} \mathrm{K})$

Format: Char $^{a}$, Char $^{b}$, Real $^{c}$, Char $^{d}$, Real $^{e}$, Char $^{f}$, Real $^{g}$, Char $^{h}$, Real $^{i}$, Char $^{j}$,

Elseif: Thermal Conductivity Function Option $=\{$ Parallel $\}$

X-Dir. Rock/Soil Grain Thermal Conductivity ${ }^{\mathrm{c}}$, Units ${ }^{\mathrm{d}}$ (W/m K), 


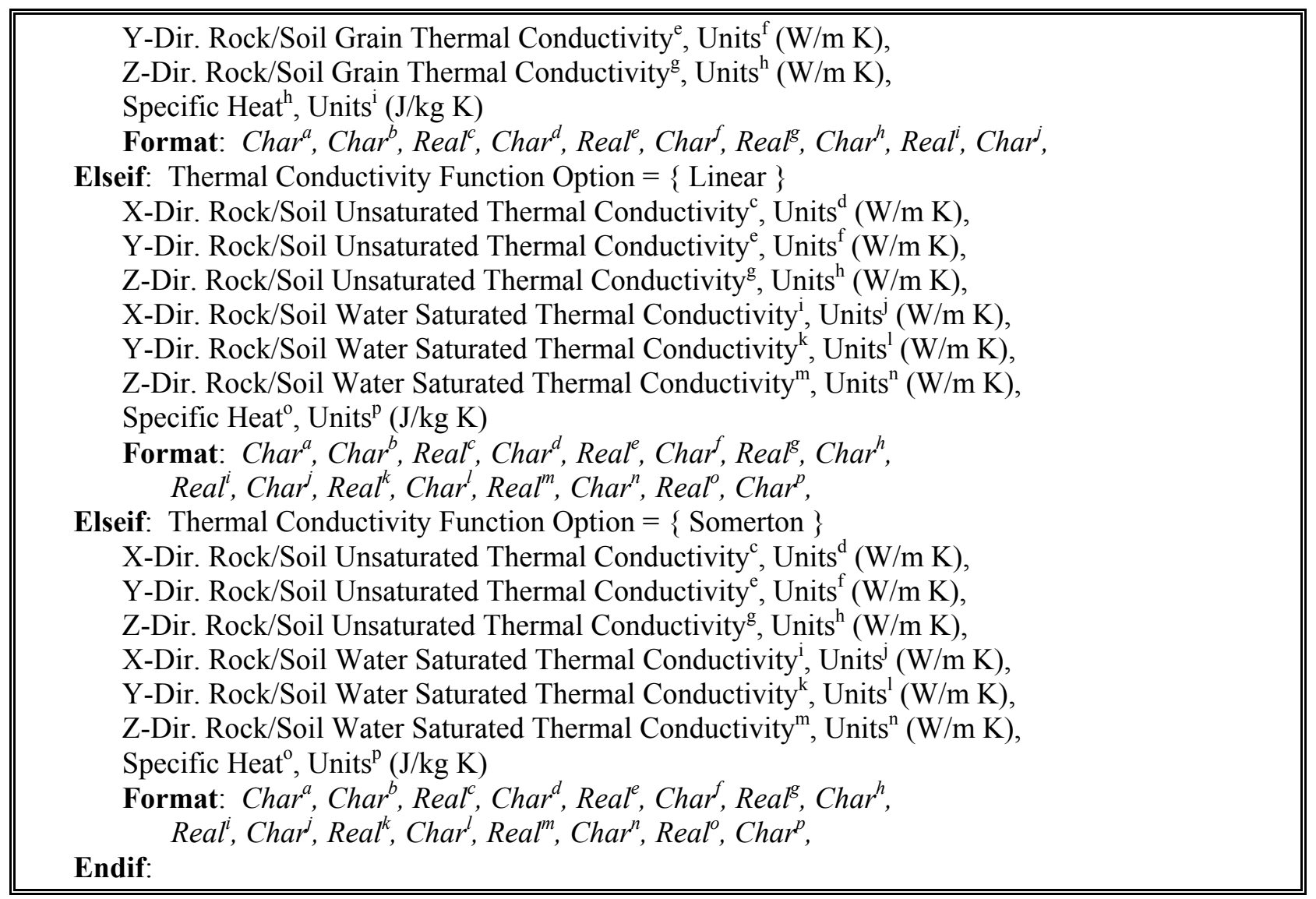

Endfor: Number of Rock/Soil Types

Endcard: Thermal Properties Card

\section{A.12.1 Thermal Properties Card Examples}

Thermal Properties Card

Sand, Somert on, 0.582, W/ m K, , , 1.13, W/ m K, , , , 700, J/kg K,

\section{A.13 Rock/Soil Zonation Card}

Card Title $^{\mathrm{a}}\{\sim$ Rock/Soil [ Zonation Card ] \}

Format: Char $^{a}$

Number of Rock/Soil Zonation Domains ${ }^{\mathrm{a}}$

Format: Integer ${ }^{a}$,

For: Number of Rock/Soil Zonation Domains

Rock/Soil Name,

I-Start Index ${ }^{\mathrm{b}}$ I-End Index ${ }^{\mathrm{c}}$,

J-Start Index ${ }^{\mathrm{d}}$, J-End Index ${ }^{\mathrm{e}}$,

K-Start Index ${ }^{\mathrm{f}}, \mathrm{K}$-End Index ${ }^{\mathrm{g}}$,

Format: Char ${ }^{a}$, Integer ${ }^{b}$, Integer ${ }^{c}$, Integer ${ }^{d}$, Integer ${ }^{e}$, Integer $^{f}$, Integer $^{g}$,

Endfor: Number of Rock/Soil Zonation Domains 
Endcard: Rock/Soil Zonation Card

\section{A.13.1 Rock/Soil Zonation Card Examples}

Rock/Soil Zonation Card

1 ,

$20 / 30$ Ot t a wa Sand, 1, 1, 1, 1, 1, 92,

$\sim$ Rock/Soil Zonation Card

7 ,

$S P 1,1,50,1,1,86,113$,

$S P 2,1,50,1,1,63,85$,

SM-ML1, 1, 50, 1, 1,69,69,

SM- SP1, 1, 50, 1, 1, 46, 62,

SP 3, 1, 50, 1, 1, 16, 45,

SW1 1, 50, 1, 1, 1, 15,

US, 2, 2, 1, 1, 71, 100,

r Rock/Soil Zonation Card

1 ,

Sand 1, 1, 20,1, 1, 1, 10,

\section{A.14 Aqueous Relative Permeability Card}

Card Title $^{\mathrm{a}}\{\sim$ Aqueous Rel [ ative Permeability Card ] \}

Format: Char $^{a}$

For: Number of Rock/Soil Types

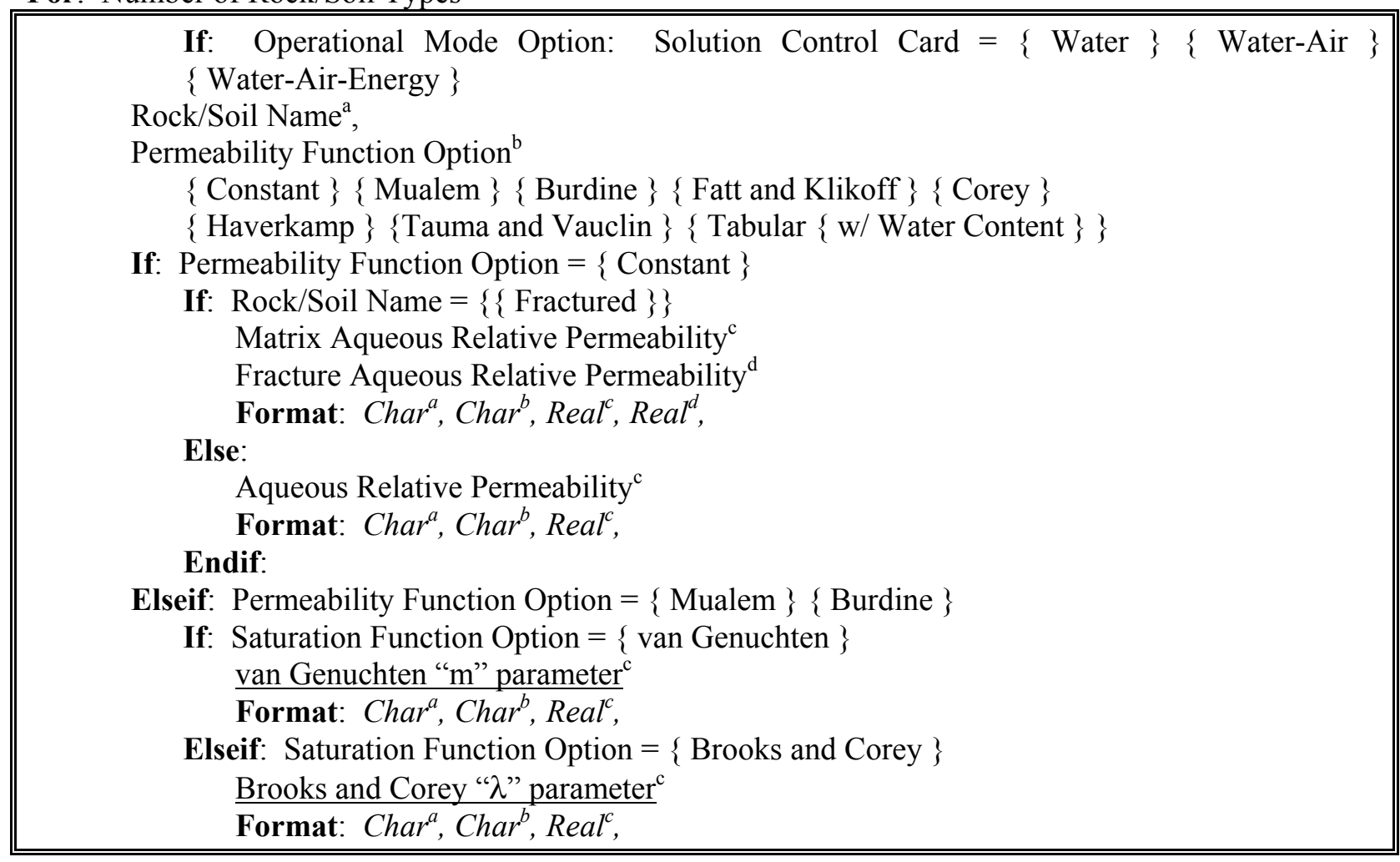




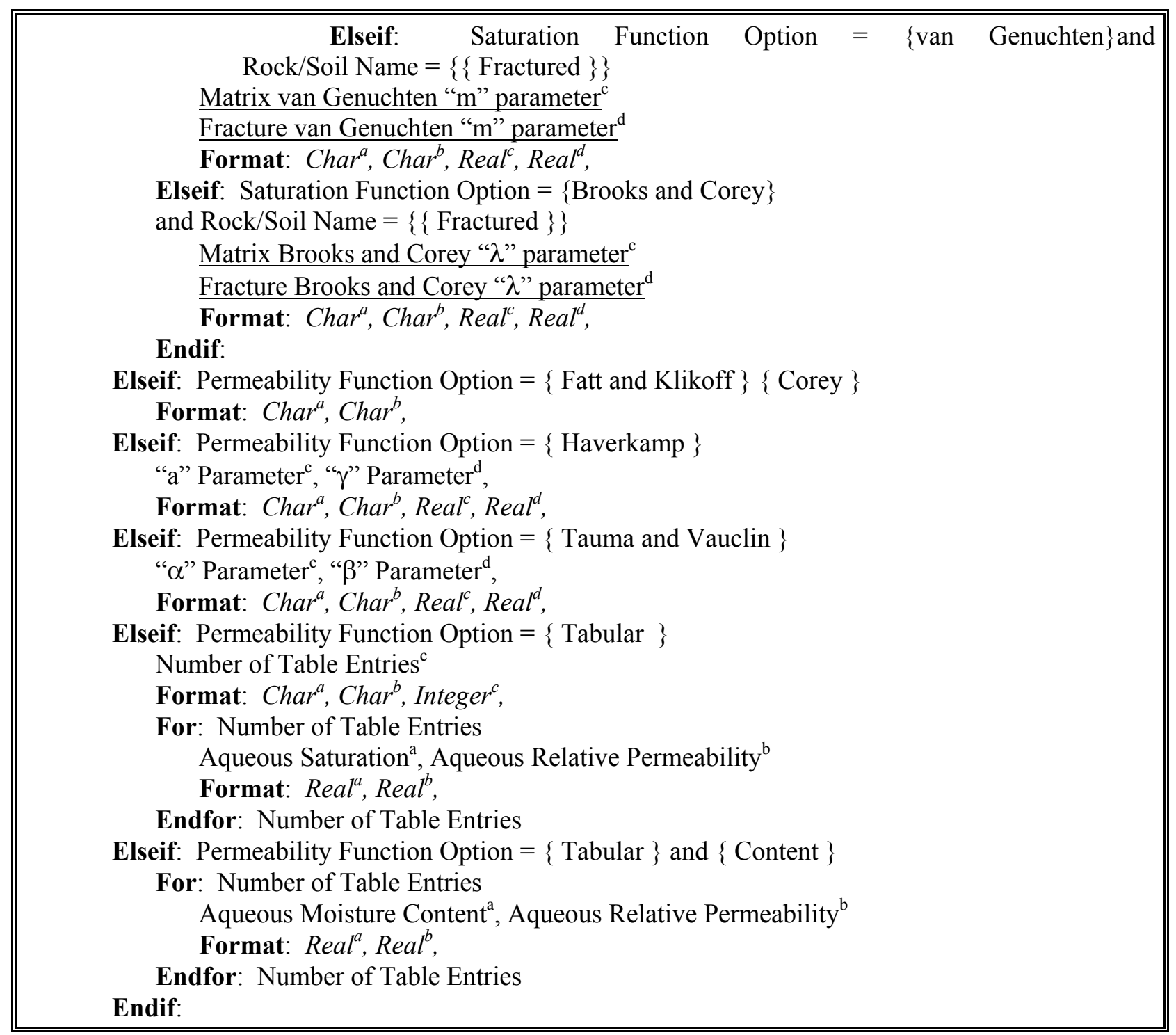

Endfor: Number of Rock/Soil Types

Endcard: Aqueous Relative Permeability Card

\section{A.14.1 Aqueous Relative Permeability Card Examples}

aqueous Relative Permeability Card

Sand, Mual em,

Aqueous Relative Permeability Card

$20 / 30$ Ot t awa Sand, Mual em, 0.56,

Aqueous Relative Permeability Card

Sand, Fatt and KI i koff,

Aqueous Relative Permeability Card

SM-ML 1, Burdi ne, , 
SW1, Burdine,

SP 3, Burdine,

SM-SP1, Burdi ne, ,

SP2, Burdine,

SP1, Burdine,

US, Touma and Vaucli n, 1.0,2.0,

\section{A.15 Surface Flux Card}

Card Title $^{\mathrm{a}}\{\sim$ Surface [ Flux Card ] $\}$

Format: Char $^{a}$

Number of Surface Flux Inputs ${ }^{\mathrm{a}}$

Format: Integer ${ }^{\mathrm{a}}$,

For: Number of Surface Flux Inputs:

If: Operational Mode Option: Solution Control Card $=\{$ Water $\}$

Surface Flux Type Option ${ }^{\mathrm{a}}$

\{Aqueous Volumetric Flux \} Aqueous Mass Flux

Elseif: Operation Mode Option: Solution Control Card $=\{$ Water-Air $\}$

Surface Flux Type Option ${ }^{\text {a }}$

\{Aqueous Volumetric Flux $\}$ Aqueous Mass Flux $\}$ Gas Volumetric Flux

\{ Gas Mass Flux \}

Elseif: Operation Mode Option: Solution Control Card $=\{$ Water-Air-Energy $\}$

Surface Flux Type Option ${ }^{\mathrm{a}}$

\{Aqueous Volumetric Flux\} \{Aqueous Mass Flux \} \{ Gas Volumetric Flux

$\{$ Gas Mass Flux $\}$ \{ Heat Flux \}

Endif:

If: Surface Flux Type Option $=\{$ Heat Flux $\}$

$\operatorname{Units}^{\mathrm{b}}(\mathrm{W}), \operatorname{Units}^{\mathrm{c}}(\mathrm{J})$,

ElseIf: Surface Flux Type Option $=\{$ Volumetric Flux $\}$

$\operatorname{Units}^{\mathrm{b}}\left(\mathrm{m}^{\wedge} 3 / \mathrm{s}\right)$, Units ${ }^{\mathrm{c}}\left(\mathrm{m}^{\wedge} 3\right)$,

Elseif: Surface Flux Type Option $=\{$ Mass Flux $\}$

Endif:

Units $^{\mathrm{b}}(\mathrm{kg} / \mathrm{s})$, Units $^{\mathrm{c}}(\mathrm{kg})$,

Surface Flux Orientation Option ${ }^{\mathrm{d}}$

\{West $\}$ East $\}$ South

\{North $\}$ Top $\}$ \{ Bottom

I-Start Index , I-End Index ${ }^{\mathrm{f}}$,

J-Start Index ${ }^{\mathrm{g}}$, J-End Index ${ }^{\mathrm{h}}$,

K-Start Index ${ }^{i}$, K-End Index ${ }^{j}$,

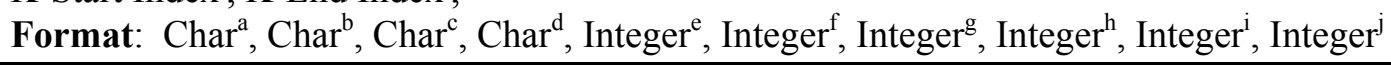

Endfor: Number of Surface Flux Inputs 


\section{Endcard:}

\section{A.15.1 Surface Flux Card Example}

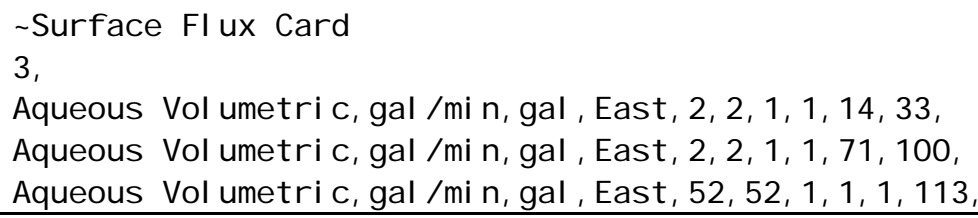

\section{A.16 Gas Relative Permeability Card}

Card Title $^{\mathrm{a}}\{\sim$ Gas Rel [ ative Permeability Card ] \}

Format: Char $^{a}$

For: Number of Rock/Soil Types

If: Operational Mode: Solution Control Card $=\{$ Water-Air $\}\{$ Water-Air-Energy $\}$

Rock/Soil Name ${ }^{\mathrm{a}}$,

Permeability Function Option ${ }^{\mathrm{b}}$

$\{$ Constant $\}\{$ Mualem $\}$ \{ Burdine $\}$ \{Fatt and Klikoff $\}$ C Corey $\}\{$ Tabular

If: Permeability Function Option $=\{$ Constant $\}$

If: Rock/Soil Name $=\{\{$ Fractured $\}\}$

Matrix Gas Relative Permeability ${ }^{\mathrm{c}}$, Fracture Gas Relative Permeability ${ }^{\mathrm{d}}$

Format: Char $^{\mathrm{a}}$, Char $^{\mathrm{b}}$, Real $^{\mathrm{c}}$, Real ${ }^{\mathrm{d}}$,

Else:

Gas Relative Permeability ${ }^{\mathrm{c}}$

Format: Char ${ }^{\mathrm{a}}$, $\mathrm{Char}^{\mathrm{b}}$, $\mathrm{Real}^{\mathrm{c}}$,

Endif:

Elseif: Permeability Function Option $=\{$ Mualem $\}$ or $\{$ Burdine $\}$

If: Saturation Function Option $=\{$ van Genuchten $\}$

van Genuchten "m" parameter

Format: Char ${ }^{\mathrm{a}}, \mathrm{Char}^{\mathrm{b}}, \mathrm{Real}^{\mathrm{c}}$,

Elseif: Saturation Function Option $=\{$ Brooks and Corey $\}$

Brooks and Corey " $\lambda$ " parameter ${ }^{c}$

Format: Char $^{\mathrm{a}}, \mathrm{Char}^{\mathrm{b}}, \mathrm{Real}^{\mathrm{c}}$,

Elseif: Saturation Function Option $=\{$ van Genuchten $\}$ and

Rock/Soil Name $=\{\{$ Fractured $\}\}$

Matrix van Genuchten " $m$ " parameter ${ }^{c}$

Fracture van Genuchten "m" parameter

Format: Char $^{\mathrm{a}}, \mathrm{Char}^{\mathrm{b}}$, Real $^{\mathrm{c}}$, ,eal $^{\mathrm{d}}$,

Elseif: Saturation Function Option $=\{$ Brooks and Corey $\}$ and

Rock/Soil Name $=\{\{$ Fractured $\}\}$

Matrix Brooks and Corey " $\lambda$ " parameter ${ }^{c}$

Fracture Brooks and Corey " $\lambda$ " parameter ${ }^{\mathrm{d}}$

Format: Char $^{\mathrm{a}}, \mathrm{Char}^{\mathrm{b}}, \underline{\text { Real }}^{\mathrm{c}}, \underline{\text { Real }}^{\mathrm{d}}$,

Endif:

Elseif: Permeability Function Option $=\{$ Tabular $\}$

Number of Table Entries ${ }^{c}$

Format: Char $^{\mathrm{a}}{ }^{,}$Char $^{\mathrm{b}}$, Integer $^{\mathrm{c}}$,

For: Number of Table Entries 


\section{Gas Saturation $^{\mathrm{a}}$, Gas Relative Permeability ${ }^{\mathrm{b}}$}

Format: Real ${ }^{\mathrm{a}}$, Real ${ }^{\mathrm{b}}$,

Endfor:

Endif:

Endfor: Number Rock/Soil Types

Endcard: Aqueous Relative Permeability Card

\section{A.16.1 Gas Relative Permeability Card Examples}

Gas Rel ative Permeability Card

Sand, Mual em,

$\sim$ Gas Rel

$20 / 30$ Ot t awa Sand, Mual em, 0.56,

Gas Relative Permeability Card

Sand, Fatt and KI i koff,

$\sim$ Gas Rel ative Permeability

SM- ML 1, Burdi ne, ,

SW1, Burdine,

\# Tabular Input

SP3, Tabular, 6,

1. 0, 1.0,

$0.8,0.64$

$0.6,0.36$

$0.4,0.16$,

$0.2,0.04$,

$0.0,0.0$,

SM-SP1, Mual e m, 0.5,

SP2, Burdine,

SP1, Burdine, ,

US, Touma and Vaucl in, 1, 0, 2,0,

\section{A.17 Solute/Fluid Interactions Card}

Card Title $^{\mathrm{a}}\{\sim$ Solute/Fluid [ Interactions Card ] $\}$

Format: Char $^{a}$

Number of Solutes

Format: Integer,

For: Number of Solutes

If: Operational Mode Option: Solution Control Card $=\{$ Water $\}$

Solute Name ${ }^{\mathrm{a}}$,

Effective Diffusion Option ${ }^{\mathrm{b}}$,

\{ Constant \}

\{Conventional \} Note: $D_{\ell}^{C}=\tau_{\ell} s_{\ell} n_{D} D_{\ell}^{C}$ 
$\{$ Empirical $\}$ Note: Kemper and van Schaik $D_{\ell_{e}}^{c}=D_{\ell}^{c}$ a e $e^{\left(b n_{D} s_{\ell}\right)}$

If: Effective Diffusion Option $=\{$ Empirical $\}$

Solute Partition Option ${ }^{\mathrm{c}}$,

$\{$ Continuous [ Concentration Dependent ] $\}$ Note:

$$
C_{\ell}=\frac{C}{n_{D} s_{\ell}+\left(1-n_{T}\right) \rho_{s} K_{s \ell}}
$$

\{ Noncontinous [ Concentration Dependent] \} Note:

$$
C_{\ell}=\frac{C}{s_{\ell} n_{D}+s_{\ell}\left(1-n_{T}\right) \rho_{s} K_{s \ell}}
$$

IfDef: Radioactive

Half-Life ${ }^{\mathrm{d}}$, Units ${ }^{\mathrm{e}}$ (s),

Format: Char $^{a}$, Char ${ }^{b}$, Char $^{c}$, Real $^{d}$, Char ${ }^{e}$,

ElseifDef: Reactive

Number of Reactions ${ }^{\mathrm{d}}$,

For: Number of Reactions:

First-Order Decay Constant ${ }^{\mathrm{e}}$, $\operatorname{Units}^{\mathrm{f}}$ (s),

Endfor: Number of Reactions

Format: Char $^{a}$, Char $^{b}$, Char $^{c}$, Integer $^{d},<$ Real $^{e}$, Char $^{f},>$

\section{EndifDef:}

Else:

Aqueous-Phase Molecular Diffusion Coefficient @ $20 \mathrm{C}^{\mathrm{c}}, \mathrm{Units}^{\mathrm{d}}\left(\mathrm{m}^{\wedge} 2 / \mathrm{s}\right)$,

Solute Partition Option ${ }^{\mathrm{e}}$,

$\{$ Continuous [ Concentration Dependent ] $\}$ Note:

$$
C_{\ell}=\frac{C}{n_{D} s_{\ell}+\left(1-n_{T}\right) \rho_{s} K_{s \ell}}
$$

\{ Noncontinous [ Concentration Dependent] \} Note:

$$
C_{\ell}=\frac{C}{s_{\ell} n_{D}+s_{\ell}\left(1-n_{T}\right) \rho_{s} K_{s \ell}}
$$

IfDef: Radioactive

Half-Life ${ }^{\mathrm{f}}$ Units $^{\mathrm{g}}$ (s),

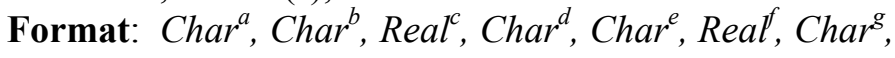

ElseifDef: Reactive

Number of Reactions ${ }^{\mathrm{f}}$,

For: Number of Reactions:

First-Order Decay Constant ${ }^{\mathrm{g}}$, Units ${ }^{\mathrm{h}}$ (s),

Endfor: Number of Reactions

Format: Char $^{a}$, Char $^{b}$, Real $^{c}$, Char $^{d}$, Char $^{e}$, Integer $^{f},<$ Real $^{g}$, Char $^{h},>$

EndifDef:

\section{Endif:}

Elseif: Operational Mode Option: Solution Control Card $=\{$ Water-Air $\}$

\{ Water-Air-Energy \}

Solute Name ${ }^{\mathrm{a}}$,

Aqueous-Phase Molecular Diffusion Coefficient @ $20 \mathrm{C}^{\mathrm{b}}, \mathrm{Units}^{\mathrm{c}}\left(\mathrm{m}^{\wedge} 2 / \mathrm{s}\right)$,

Gas-Phase Molecular Diffusion Coefficient @20 C ${ }^{\mathrm{d}}$, Units ${ }^{\mathrm{e}}\left(\mathrm{m}^{\wedge} 2 / \mathrm{s}\right)$,

Gas-Aqueous Partition Function Option ${ }^{\mathrm{f}}$

\{ Constant \}

\{ Temperature Dependent $\}$ Note:

$$
\ln \left(K_{g \ell}\right)=a+\frac{b}{T}+c \ln (T)+d T+e T^{2}
$$

If: Gas-Aqueous Partition Function Option $=\{$ Constant $\}$ 


\begin{tabular}{|c|}
\hline 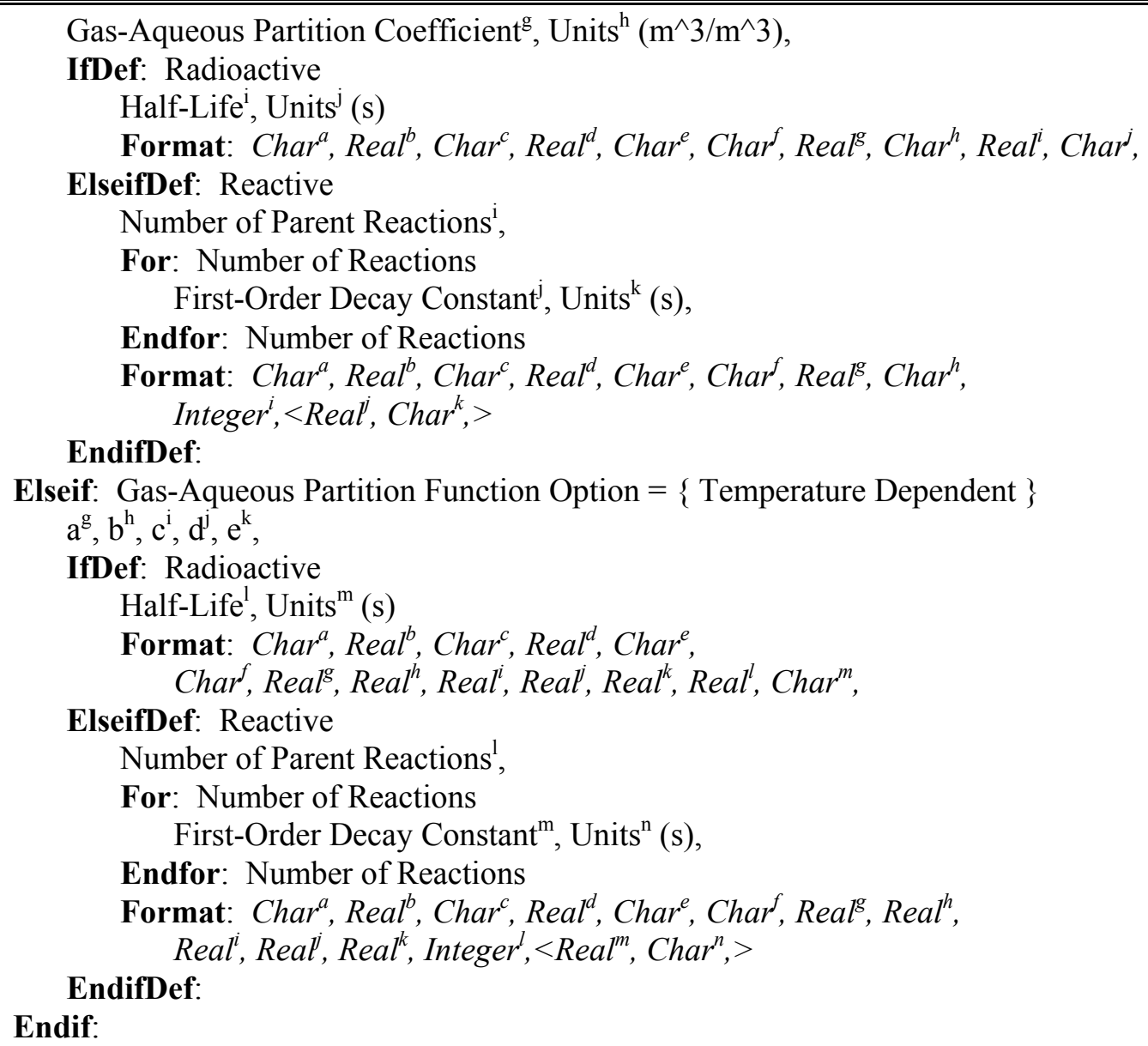 \\
\hline
\end{tabular}

\section{Endcard:}

\section{A.17.1 Solute/Fluid Interactions Card Examples}

Sol ute/FI uid Interaction Card

1 ,

TCE, Conventional $, 9.6283 \mathrm{e}-6, \mathrm{~cm}^{\wedge} 2 / \mathrm{s}$, Cont i nuous, $1000, \mathrm{yr}$,

0 ,

Sol ute/FIuid I nteraction Card

4 ,

Tc-99, Empirical, Noncontinuous, 1. e20, yr,

U-238, Empirical, Noncontinuous, 1. e20, yr,

Np-237, Empirical, Noncontinuous, 1. e20, yr,

Pu-239, Empirical, Noncontinuous, 1. e20, yr,

0 ,

Solute/FIuid Interaction Card

7 ,

Dithionite, Conventional, 1, e-5, cm^2/s, Conti nuous, 2, 5, hr, 18, hr,

Sulfite, Conventional, 1. e- 5, cm^2/s, Conti nuous, 0 ,

Bisulfite, Conventional, 1, e- $5, \mathrm{~cm}^{\wedge} 2 / \mathrm{s}$, Continuous, 0 , 


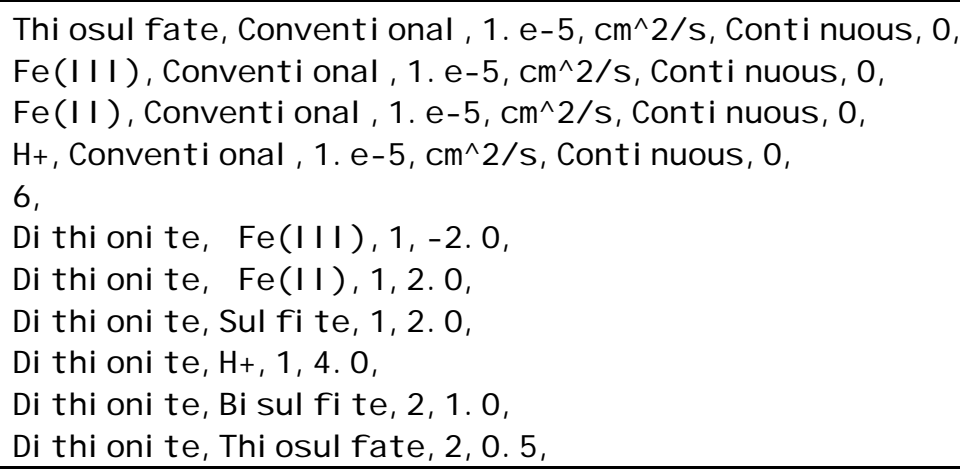

\section{A.18 Solute/Porous Media Interactions Card}

Card Title $^{\mathrm{a}}\{\sim$ Solute/Porous [ Media Interactions Card ] $\}$

Format: Char $^{a}$

For: Number of Rock/Soil Types

Rock/Soil Name ${ }^{a}$,

Longitudinal Dispersivity ${ }^{\mathrm{b}}, \operatorname{Units}^{\mathrm{c}}(\mathrm{m})$,

Transverse Dispersivity ${ }^{\mathrm{d}}, \operatorname{Units}^{\mathrm{e}}(\mathrm{m})$

Format: Char $^{a}$, Real $^{b}$, Char $^{c}$, Real $^{d}$, Char $^{e}$,

For: Number of Solutes

Solute Name ${ }^{\mathrm{a}}$,

Solid-Aqueous Partition Coefficient ${ }^{\mathrm{b}}$, Units ${ }^{\mathrm{c}}\left(\mathrm{m}^{\wedge} 3 / \mathrm{kg}\right)$

If: Solute Partition Option: Solute/Fluid Interactions Card $=\{$ Concentration Dependent $\}$

Dependent Solute Name ${ }^{\mathrm{d}}$,

"a" Parameter", "b" Parameter,

If: Effective Diffusion Option: Solute/Fluid Interactions Card $=\{$ Empirical $\}$

Aqueous Molecular Diffusion Coefficient ${ }^{\mathrm{d}}$, Units ${ }^{\mathrm{e}}\left(\mathrm{m}^{\wedge} 2 / \mathrm{s}\right), \mathrm{a}^{\mathrm{f}}, \mathrm{b}^{\mathrm{g}}$,

Format: Char $^{a}$, Real $^{b}$, Char $^{c}$, Char $^{d}$, Real ${ }^{e}$, Real $^{f}$, Real $^{g}$, Char $^{h}$, Real $^{i}$, Real $^{j}$, Else:

Format: Char $^{a}$, Real $^{b}$, Char $^{c}$, Char $^{d}$, Real $^{e}$, Real

\section{Endif:}

Else:

If: Effective Diffusion Option: Solute/Fluid Interactions Card $=\{$ Empirical $\}$

Aqueous Molecular Diffusion Coefficient ${ }^{\mathrm{d}}, \operatorname{Units}^{\mathrm{e}}\left(\mathrm{m}^{\wedge} 2 / \mathrm{s}\right), \mathrm{a}^{\mathrm{f}}, \mathrm{b}^{\mathrm{g}}$,

Format: Char $^{a}$, Real $^{b}$, Char $^{c}$, Real $^{d}$, Char $^{e}$, Real $^{f}$, Real $^{g}$,

Else:

Format: Char $^{a}$, Real $^{b}$, Char $^{c}$,

\section{Endif:}

Endif:

Endfor: Number of Solutes

Endfor: Number of Rock/Soil Types

Endcard:

\section{A.18.1 Solute/Porous Media Interaction Card Examples}

Sol ute/Porous Media Interaction Card

SP 1, , , , 
TCE, $0,0, \mathrm{~m}^{\wedge} 3 / \mathrm{kg}$,

$S P 2, \ldots,$,

TCE, 0,0, $\mathrm{m}^{\wedge} 3 / \mathrm{kg}$,

SM-ML $1,,,$, ,

$T C E, 0,0, m^{\wedge} 3 / \mathrm{kg}$,

SM-SP1, , , ,

$T C E, 0,0, m^{\wedge} 3 / \mathrm{kg}$,

$S P 3, \ldots, \ldots$

TCE, $0,0, \mathrm{~m}^{\wedge} 3 / \mathrm{kg}$,

$S W 1, \ldots, \ldots$

TCE, $0,0, \mathrm{~m}^{\wedge} 3 / \mathrm{kg}$,

US, , , ,

$T C E, 0,0, m^{\wedge} 3 / \mathrm{kg}$,

Sol ute/Porous Media Interaction Card

Backfill Soil, , , ,

$\mathrm{Tc}-99,0 ., c \mathrm{~m}^{\wedge} 3 / \mathrm{g}, 7.8894 \mathrm{e}-2, \mathrm{~m}^{\wedge} 2 / \mathrm{yr}, 0.005,10$.

U. $238,0.67, \mathrm{~cm}^{\wedge} 3 / \mathrm{g}, 7.8894 \mathrm{e}-2, \mathrm{~m}^{\wedge} 2 / \mathrm{yr}, 0.005,10$, ,

$\mathrm{Np}-237,3.0, \mathrm{~cm}^{\wedge} 3 / \mathrm{g}, 7.8894 \mathrm{e}-2, \mathrm{~m}^{\wedge} 2 / \mathrm{yr}, 0.005,10$, ,

Pu-239,21.0, cm^3/g, 7.8894e-2, $\mathrm{m}^{\wedge} 2 / \mathrm{yr}, 0.005,10$,

Hanford Sand, , , ,

$T c-99,0 ., c m^{\wedge} 3 / g, 7.8894 \mathrm{e}-2, \mathrm{~m}^{\wedge} 2 / \mathrm{yr}, 0.005,10 .$,

U. $238,0.67, \mathrm{~cm}^{\wedge} 3 / \mathrm{g}, 7.8894 \mathrm{e}-2, \mathrm{~m}^{\wedge} 2 / \mathrm{yr}, 0.005,10$, ,

$\mathrm{Np}-237,3.0, \mathrm{~cm}^{\wedge} 3 / \mathrm{g}, 7.8894 \mathrm{e}-2, \mathrm{~m}^{\wedge} 2 / \mathrm{yr}, 0.005,10$.,

Pu-239,21.0,cm^3/g, 7.8894e-2, $\mathrm{m}^{\wedge} 2 / \mathrm{yr}, 0.005,10$,

Hanford Gravel, , , ,

$T c-99,0 ., c m^{\wedge} 3 / g, 7.8894 \mathrm{e}-2, \mathrm{~m}^{\wedge} 2 / \mathrm{yr}, 0.005,10$,

U. $238,0.67, \mathrm{~cm}^{\wedge} 3 / \mathrm{g}, 7.8894 \mathrm{e}-2, \mathrm{~m}^{\wedge} 2 / \mathrm{yr}, 0.005,10$, ,

$\mathrm{Np}-237,3.0, \mathrm{~cm}^{\wedge} 3 / \mathrm{g}, 7.8894 \mathrm{e}-2, \mathrm{~m}^{\wedge} 2 / \mathrm{yr}, 0.005,10$.,

Pu-239, 21, 0, cm^3/g, 7.8894e-2, $\mathrm{m}^{\wedge} 2 / \mathrm{yr}, 0.005,10$,

Ringold Formation, , , ,

Tc-99,0.,cm^3/g, 7.8894e-2, $\mathrm{m}^{\wedge} 2 / \mathrm{yr}, 0.005,10$,

U. $238,0.67, \mathrm{~cm}^{\wedge} 3 / \mathrm{g}, 7.8894 \mathrm{e}-2, \mathrm{~m}^{\wedge} 2 / \mathrm{yr}, 0.005,10$,

$\mathrm{Np}-237,3,0, \mathrm{~cm}^{\wedge} 3 / \mathrm{g}, 7.8894 \mathrm{e}-2, \mathrm{~m}^{\wedge} 2 / \mathrm{yr}, 0.005,10$, ,

Pu-239, 21.0, c m^3/g, 7.8894e-2, $\mathrm{m}^{\wedge} 2 / \mathrm{yr}, 0.005,10$,

Gl ass Waste, ,.,

$T c-99,0 ., c m^{\wedge} 3 / g, 3.1557 \mathrm{e}-7, \mathrm{~m}^{\wedge} 2 / \mathrm{yr}, 1,0 .$,

U. $238,0 ., c m^{\wedge} 3 / g, 3.1557 \mathrm{e}-7, \mathrm{~m}^{\wedge} 2 / \mathrm{yr}, 1,0 .$,

$\mathrm{Np}-237,0, c \mathrm{~m}^{\wedge} 3 / \mathrm{g}, 3.1557 \mathrm{e}-7, \mathrm{~m}^{\wedge} 2 / \mathrm{yr}, 1,0 .$,

Pu-239, 0., cm^3/g, 3.1557e- $7, \mathrm{~m}^{\wedge} 2 / \mathrm{yr}, 1 ., 0 .$,

Sol ute/Porous Media Interaction Card

Soil, , , ,

Dithionite, $0,0, \mathrm{~m}^{\wedge} 3 / \mathrm{kg}$,

Sulfite, 0,0, $\mathrm{m}^{\wedge} 3 / \mathrm{kg}$,

Bisulfite, 0.0, $\mathrm{m}^{\wedge} 3 / \mathrm{kg}$,

Thi osulfate, $0.0, \mathrm{~m}^{\wedge} 3 / \mathrm{kg}$,

$\mathrm{Fe}(\mathrm{I} \mid \mathrm{l}), 0.0, \mathrm{~m}^{\wedge} 3 / \mathrm{kg}$,

$\mathrm{Fe}(\mathrm{II}), 0,0, \mathrm{~m}^{\wedge} 3 / \mathrm{kg}$,

$\mathrm{H}+, 0,0, \mathrm{~m}^{\wedge} 3 / \mathrm{kg}$,

\section{A.19 Reactive Transport Control Card}


Card Title $^{\mathrm{a}}\{\sim$ Reactive [ Transport Control Card ] $\}$

Format: Char $^{a}$

Reactive Transport Execution Mode Option ${ }^{\mathrm{a}}$

$\{$ Normal \}

Format: Char $^{a}$,

Reactive Transport Solution Mode Option ${ }^{\mathrm{a}}$

\{ Fully Coupled \}

\{Operator Split \}

Format: Char $^{a}$,

Aitken extrapolation scheme switch ${ }^{\mathrm{a}}$, Column scan switch ${ }^{\mathrm{b}}$, Non-reacting solver switch $^{\mathrm{c}}$, Effective reaction area switch ${ }^{\mathrm{d}}$, Texture solver / transport solver coupling switch, Porosity update with precipitation/dissolution ${ }^{\mathrm{f}}$, Lower boundary condition switch (if $0, \mathrm{dC} / \mathrm{dz}=0$, if $\left.1, \mathrm{C}=0\right)^{\mathrm{g}}$, Water consumption in chemical reactions / water flow coupling switch ${ }^{\mathrm{h}}$, Gas consumption in chemical reactions / gas flow coupling switch ${ }^{\mathrm{i}}$, Diffusive/Dispersive time step limiting switch ${ }^{\mathrm{j}}$,

Format: Integer $(0 \text { or } 1)^{\mathrm{a}}$, Integer $(0 \text { or } 1)^{\mathrm{b}}$, Integer $(0 \text { or } 1)^{\mathrm{c}}$, Integer $(0 \text { or } 1)^{\mathrm{d}}$, Integer $(0 \text { or } 1)^{\mathrm{e}}$, Integer $(0$ or 1$)^{\mathrm{f}}$, Integer $(0 \text { or } 1)^{\mathrm{g}}$, Integer $(0 \text { or } 1)^{\mathrm{h}}$, Integer $(0 \text { or } 1)^{\mathrm{i}}$, Integer $(0 \text { or } 1)^{\mathrm{j}}$

Log Maximum Concentration ${ }^{\mathrm{a}}$, Crank-Nicolson parameter $^{\mathrm{b}}$, Error in non-reacting species ${ }^{\mathrm{c}}$, Error in equation point solver $^{\mathrm{d}}$, Error in transport point solver ${ }^{\mathrm{e}}$, Error in texture solver ${ }^{\mathrm{f}}$, Error in normal concentration solver ${ }^{\mathrm{g}}$

Format: Real ${ }^{\mathrm{a}}, \mathrm{Real}^{\mathrm{b}}$, Real ${ }^{\mathrm{c}}, \mathrm{Real}^{\mathrm{d}}$, Real ${ }^{\mathrm{e}}, \mathrm{Real}^{\mathrm{f}}, \mathrm{Real}^{\mathrm{g}}$

\section{A.19.1 Reactive Transport Control Card Example}

Reactive Transport Control card

Nor mal,

Ful I y coupled,

$0,0,0,0,1,0,0,0,0,0$,

1. $0 E+00,1.0 E+00,1.0 E-06,1.0 E-07,1.0 E-03,5.0 E-03,2.0 E-03$,

\section{A.20 Aqueous Species Card}

Card Title ${ }^{\mathrm{a}}\{\sim$ Aqueous Species [ Card ] \}

Format: Char $^{a}$

Number of Aqueous Species ${ }^{\mathrm{a}}$

Format: Integer ${ }^{\mathrm{a}}$

For: Number of Aqueous Species

Name of Aqueous Species ${ }^{a}$, Charge ${ }^{b}$, Hard Core Diameter ${ }^{c}$, Number of Elements ${ }^{d}$

Format: Char $^{\mathrm{a}}$, Real ${ }^{\mathrm{b}}$, Real ${ }^{\mathrm{c}}$, Integer ${ }^{\mathrm{d}}$

For: Number of Elements in Aqueous Species

Stoichiometric Coefficient of Element ${ }^{\mathrm{a}}$, Element Name ${ }^{\mathrm{b}}$

Format: Real ${ }^{\mathrm{a}}$, Char $^{\mathrm{b}}$,

Endfor: Number of Elements in Aqueous Species 
Endfor: Number of Aqueous Species

Endcard:

\section{A.20.1 Aqueous Species Card Example}

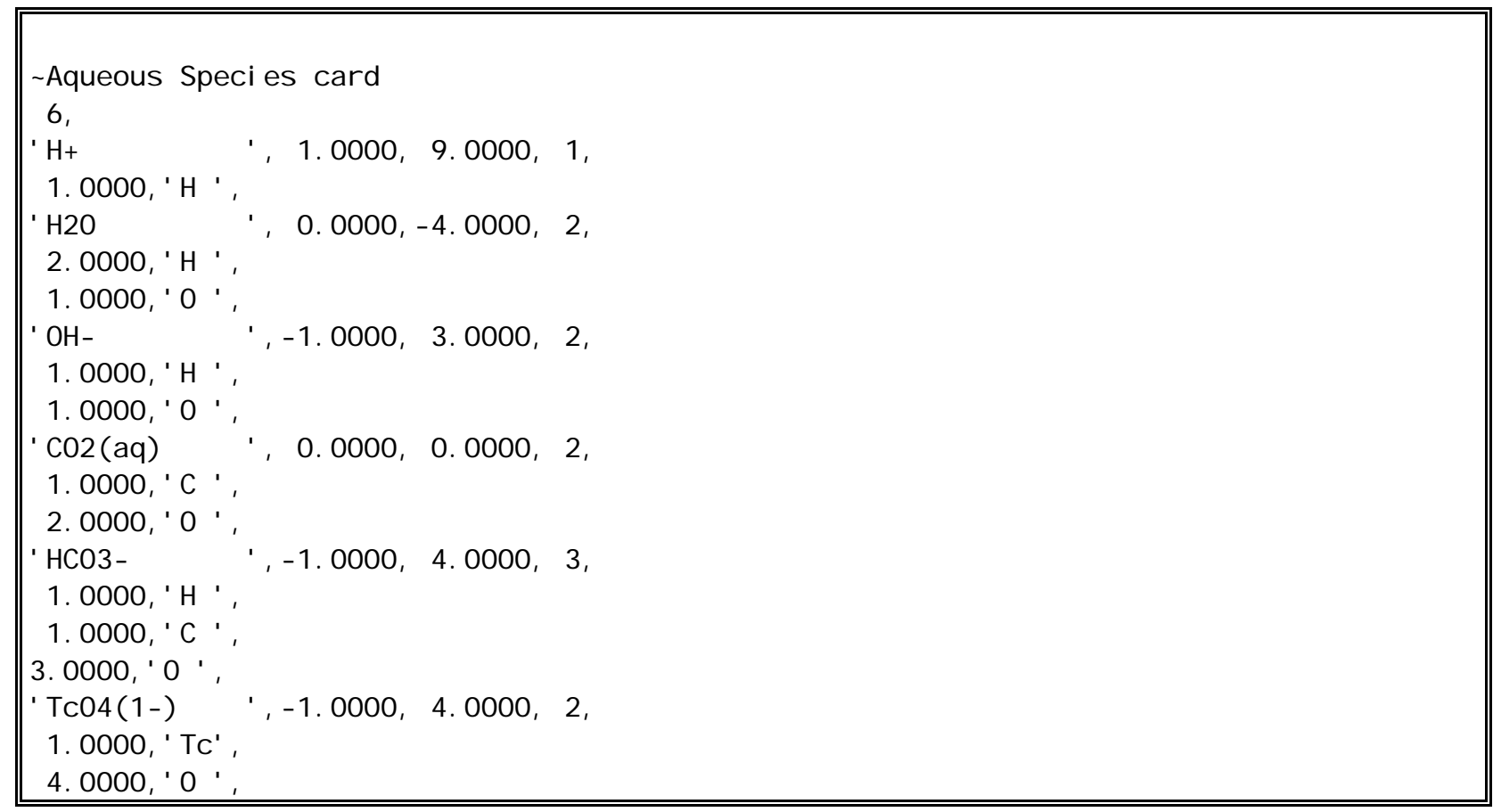

\section{A.21 Gas Species Card}

Card Title $^{\mathrm{a}}\{\sim$ Gas Species [ Card ] $\}$

Format: Char $^{a}$

Number of Gas Species ${ }^{\mathrm{a}}$

Format: Integer ${ }^{\mathrm{a}}$

For: Number of Gas Species

Name of Gas Species ${ }^{\mathrm{a}}$, Name of Equilibrium Aqueous Species ${ }^{\mathrm{b}}$, Gas Species Concentration

Dependency (fixed or transient) ${ }^{\mathrm{c}}$, Gas Diffusion Coefficient $\left(\mathrm{m}^{2} \mathrm{~s}^{-1}\right)^{\mathrm{d}}$,

Number of Elements in Gaseous Species ${ }^{\mathrm{e}}$

Format: Char $^{\mathrm{a}}, \mathrm{Char}^{\mathrm{b}}, \mathrm{Char}^{\mathrm{c}}$, Real ${ }^{\mathrm{d}}$, Integer ${ }^{\mathrm{e}}$

For: Number of Elements in Gas Species

Stoichiometric Coefficient of Element ${ }^{\mathrm{a}}$, Element Name ${ }^{\mathrm{b}}$

Format: Real ${ }^{\mathrm{a}}$, Char ${ }^{\mathrm{b}}$,

Endfor: Number of Elements in Gas Species

Number of Terms in Henry's Coefficient vs. Temperature Curve Fit ${ }^{\text {a }}$

Format: Integer ${ }^{\mathrm{a}}$

For: Number of Terms in Henry's Coefficient vs. Temperature Curve Fit log Henry's Law Equilibrium Coefficient Term (M/atm), 
Format: Real $^{\mathrm{a}}$

Endfor: Number of Terms in Henry's Coefficient vs. Temperature Curve Fit

Endfor: Number of Gas Species

Endcard:

\section{A.21.1 Gas Species Card Example}

\begin{tabular}{|c|c|}
\hline 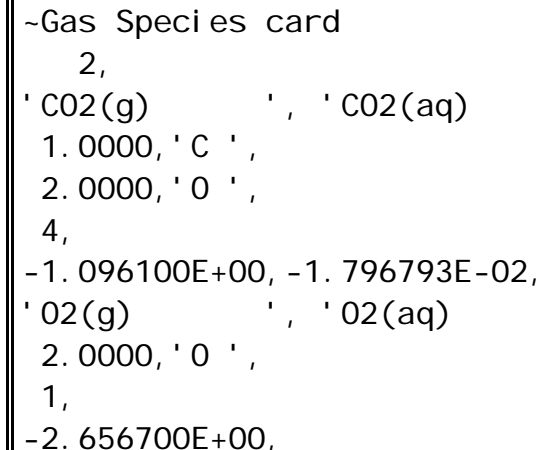 & $\begin{array}{l}\text { ', 'transient', } 1.69443 E-06,2, \\
\begin{array}{l}\text { 1. } 321931 E-04,-3.982381 E-07, \\
\text { ', 'fixed', } 2.40000 \text { E-06, 1, }\end{array}\end{array}$ \\
\hline
\end{tabular}

\section{A.22 Components Card}

Card Title $^{\mathrm{a}}\{\sim$ Components [ Card ] $\}$

Format: Char $^{a}$

Number of Components ${ }^{\mathrm{a}}$

Format: Integer ${ }^{\mathrm{a}}$

For: Number of Components

Name of Component ${ }^{\mathrm{a}}$, Molecular Mass $\left(\mathrm{kg} \mathrm{kmol}^{-1}\right)^{\mathrm{b}}$, Number of Aqueous Species ${ }^{\mathrm{c}}$,

Format: Char $^{\mathrm{a}}$, Real $^{\mathrm{b}}$, Integer ${ }^{\mathrm{c}}$

For: Number Aqueous Species

Stoichiometric Coefficient of Aqueous Species ${ }^{\mathrm{a}}$, Aqueous Species Name

Format: Real ${ }^{\mathrm{a}}$, Char ${ }^{\mathrm{b}}$,

Endfor: Number of Aqueous Species

Endfor: Number of Aqueous Species

\section{Endcard:}




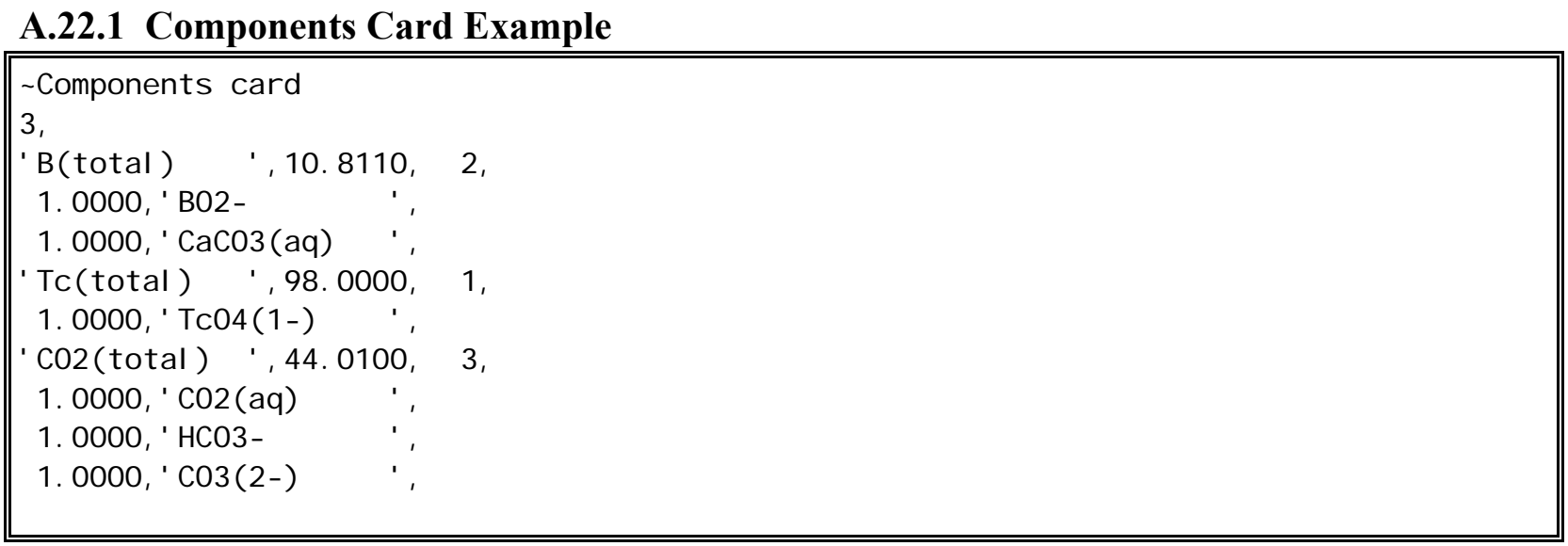

\section{A.23 Solid Species Card}

Card Title $^{\mathrm{a}}\{\sim$ Solid Species [ Card ] $\}$

Format: Char $^{a}$

Number of Solid Species ${ }^{\mathrm{a}}$

Format: Integer ${ }^{\mathrm{a}}$

For: Number of Solid Species

Name of Solid Species ${ }^{\mathrm{a}}$, Mass Density $\left(\mathrm{Mg} \mathrm{m}^{-3}\right)$, Number of Elements ${ }^{\mathrm{c}}$

Format: Char $^{\mathrm{a}}$, Real ${ }^{\mathrm{b}}$, Real ${ }^{\mathrm{c}}$, Integer ${ }^{\mathrm{d}}$

For: Number of Elements in Solid Species

Stoichiometric Coefficient of Element ${ }^{\mathrm{a}}$, Element Name ${ }^{\mathrm{b}}$

Format: Real ${ }^{\mathrm{a}}$, Char ${ }^{\mathrm{b}}$,

Endfor: Number of Elements in Solid Species

Endfor: Number of Solid Species

\section{Endcard:}

\section{A.23.1 Solid Species Card Example}

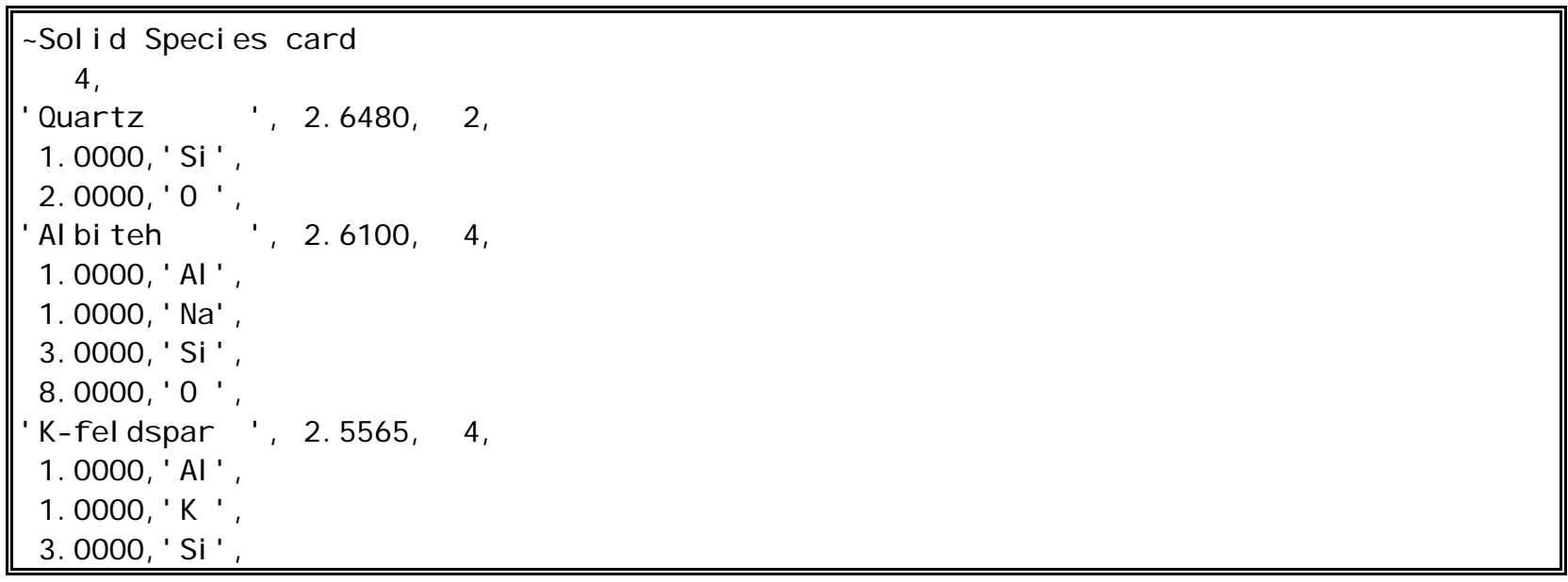




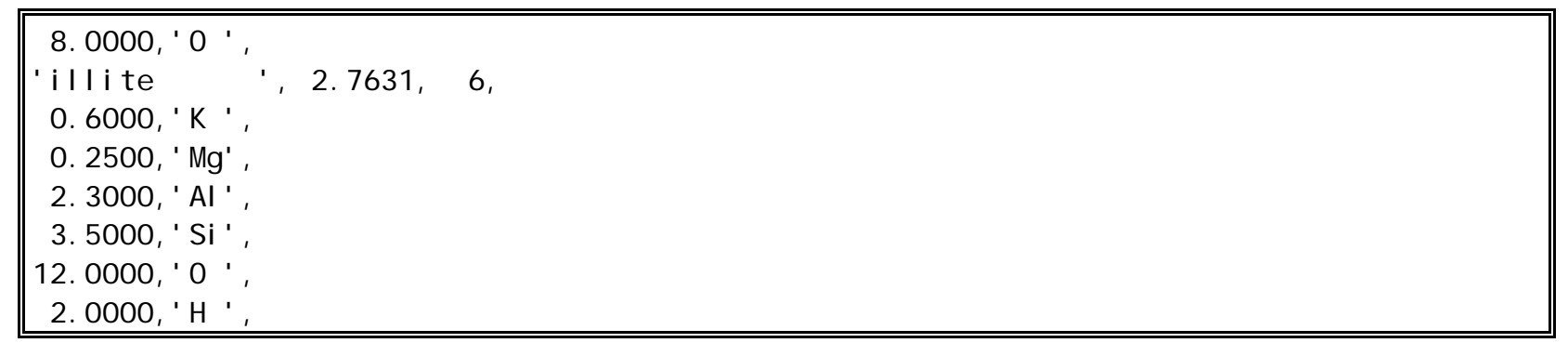

\section{A.24 Fast Reactions Card}

Card Title $^{\mathrm{a}}\{\sim$ Fast Reactions [ Card ] $\}$

Format: Char $^{a}$

Number of Fast Reactions ${ }^{\text {a }}$

Format: Integer ${ }^{\mathrm{a}}$

For: Number of Fast Reactions

Number of Aqueous Species ${ }^{\mathrm{a}}$

Format: Integer ${ }^{\mathrm{a}}$

For: Number Aqueous Species

Stoichiometric Coefficient of Aqueous Species ${ }^{a}$, Aqueous Species Name

Format: Real ${ }^{\mathrm{a}}$, Char $^{\mathrm{b}}$,

Endfor: Number of Aqueous Species

Number of Coefficients in Equilibrium Constant vs. temperature polynomial function ${ }^{\mathrm{a}}$,

Exponential Factor of Equilibrium Reaction ${ }^{\mathrm{b}}$

Format: Integer ${ }^{\mathrm{a}}$, Real $^{\mathrm{b}}$

For: Number of Coefficients in Equilibrium Constant vs. temperature polynomial function Coefficient in Equilibrium Constant vs. temperature polynomial function ${ }^{\mathrm{a}}$

Format: Real ${ }^{\mathrm{a}}, \mathrm{Real}^{\mathrm{a}}, \mathrm{Real}^{\mathrm{a}}, \ldots$

Endfor:

Endfor: Number of Fast Reactions

\section{Endcard:}

\section{A.24.1 Fast Reactions Card Example}

\begin{tabular}{|c|c|}
\hline 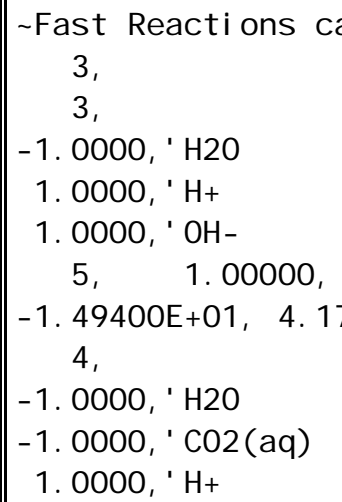 & $\begin{array}{l}1, \\
\vdots, \\
0 E-02,-1.91700 E-04,4.95000 E-07,-6.15900 E-10, \\
\therefore, \\
\therefore,\end{array}$ \\
\hline
\end{tabular}




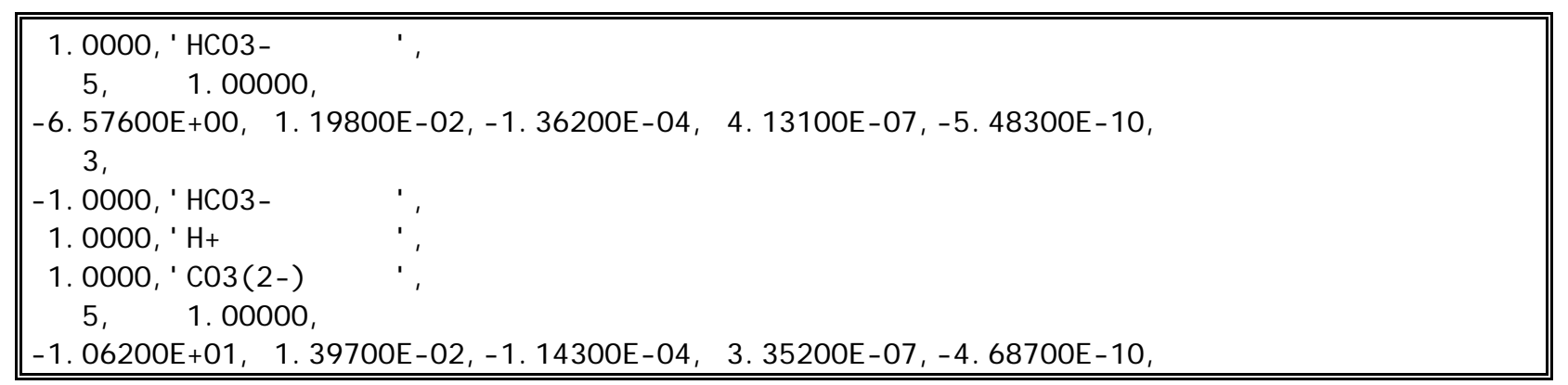

\section{A.25 Kinetic Aqueous Reactions Card}

Card Title $^{\mathrm{a}}\{\sim$ Kinetic Aqueous Reactions [ Card ] $\}$

Format: Char $^{a}$

Number of Kinetic Aqueous Reactions ${ }^{\text {a }}$

Format: Integer ${ }^{\mathrm{a}}$

For: Number of Kinetic Aqueous Reactions

Endfor:

Number of Aqueous Species ${ }^{\mathrm{a}}$

Format: Integer ${ }^{\mathrm{a}}$

For: Number Aqueous Species

Stoichiometric Coefficient of Aqueous Species ${ }^{\mathrm{a}}$, Aqueous Species Name

Format: Real ${ }^{\mathrm{a}}$, Char ${ }^{\mathrm{b}}$,

Endfor: Number of Aqueous Species

Number of Coefficients in Equilibrium Constant vs. temperature polynomial function ${ }^{\mathrm{a}}$,

Exponential Factor of Equilibrium Reaction ${ }^{\mathrm{b}}$

Format: Integer ${ }^{\mathrm{a}}$, Real ${ }^{\mathrm{b}}$

For: Number of Coefficients in Equilibrium Constant vs. temperature polynomial function

Coefficient in Equilibrium Constant vs. temperature polynomial function ${ }^{\mathrm{a}}$

Format: Real ${ }^{\mathrm{a}}, \mathrm{Real}^{\mathrm{a}}, \mathrm{Real}^{\mathrm{a}}, \ldots$

Endfor:

Rate Constant of Aqueous Kinetic Reaction ${ }^{\mathrm{a}}$

Format: Real $^{\mathrm{a}}$

\section{Endcard:}

\section{A.25.1 Kinetic Aqueous Reactions Card Example}

rinetic Aqueous Reactions card

0 , 


\section{A.26 Slow Reactions Card}

Card Title $^{\text {a }}\{\sim$ Slow Reactions [ Card ] $\}$

Format: Char $^{a}$

Number of Slow Reactions ${ }^{\text {a }}$

Format: Integer ${ }^{\mathrm{a}}$

For: Number of Slow Reactions

Mass Action Law Type ${ }^{\mathrm{a}}\{$ Full $=1\}\{$ Reduced $=-1\}\{$ Glass $=0\}$,

Number of Solid Species in Slow Reaction ${ }^{\mathrm{b}}\{1$ or 2$\}$

Format: Integer ${ }^{\mathrm{a}}$, Integer ${ }^{\mathrm{b}}$

For: Number of Solid Species in Slow Reaction

Stoichiometric Coefficient of Solid Species in Slow Reaction ${ }^{a}$, Name of Solid Species ${ }^{b}$

Format: Real ${ }^{\mathrm{a}}$, Character $^{\mathrm{b}}$

EndFor: Number of Solid Species in Slow Reaction

Number of Aqueous Species ${ }^{\mathrm{a}}$

Format: Integer ${ }^{\mathrm{a}}$

For: Number of Aqueous Species

Stoichiometric Coefficient of Aqueous Species ${ }^{\mathrm{a}}$, Aqueous Species Name ${ }^{\mathrm{b}}$,

Species Participation in Reduced Mass Action Reaction Rate Calculation,

$\{-1=$ Forward Rate Only $\},\{0=$ Neither $\},\{1=$ Equilibrium Only $\}$

Stoichiometric Coefficient of Aqueous Species Used in Equilibrium Calculations ${ }^{\mathrm{d}}$

Format: Real ${ }^{\mathrm{a}}$, $\mathrm{Char}^{\mathrm{b}}$, Real ${ }^{\mathrm{c}}$, Real ${ }^{\mathrm{d}}$,

Endfor: Number of Aqueous Species

Rate Constant of Solid Reaction ${ }^{\mathrm{a}}$, Activation Energy of Solid Reaction ${ }^{\mathrm{b}}$, Exponential Factor of Solid Reaction $^{\mathrm{c}}$, Exponential Factor of $\mathrm{pH}$ dependence of dissolution Reaction ${ }^{\mathrm{d}}$, Number of

Coefficients in Equilibrium Constant vs. temperature polynomial Function ${ }^{\mathrm{e}}$,

Format: Real ${ }^{\mathrm{a}}$, Real ${ }^{\mathrm{b}}, \mathrm{Real}^{\mathrm{c}}, \mathrm{Real}^{\mathrm{d}}$, Integer ${ }^{\mathrm{e}}$

For: Number of Coefficients in Equilibrium Constant vs. temperature polynomial function

Coefficient in Equilibrium Constant vs. temperature polynomial function ${ }^{\mathrm{a}}$

Format: Real ${ }^{\mathrm{a}}, \mathrm{Real}^{\mathrm{a}}, \mathrm{Real}^{\mathrm{a}}, \ldots$

Endfor:

Endfor: Number of Slow Reactions

Endcard:

\section{A.26.1 Slow Reactions Card Example}

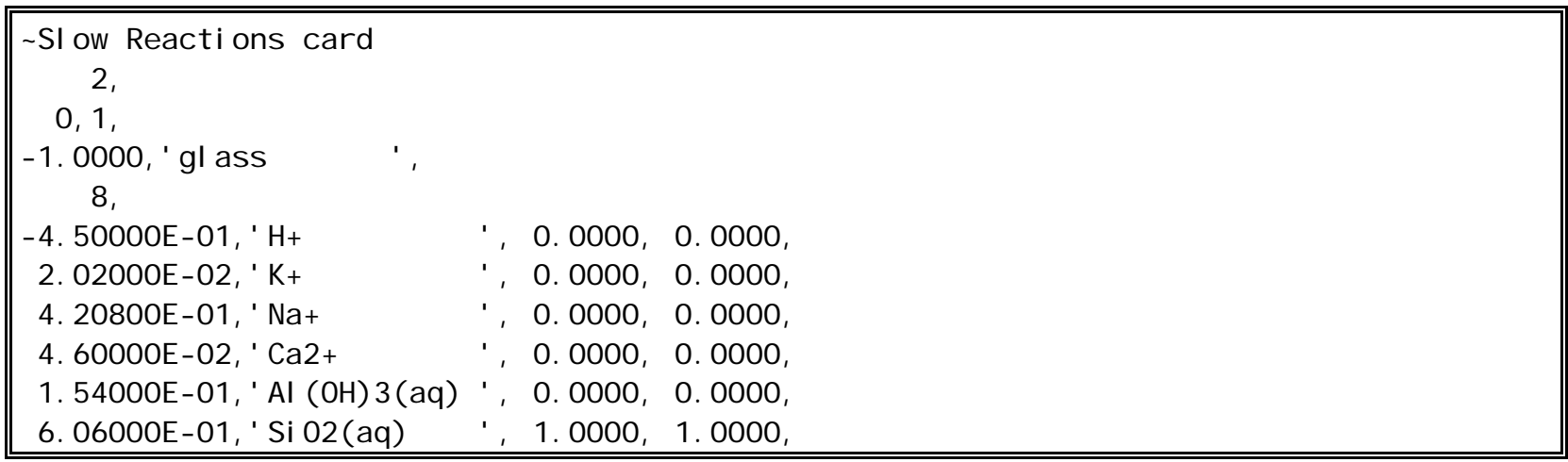




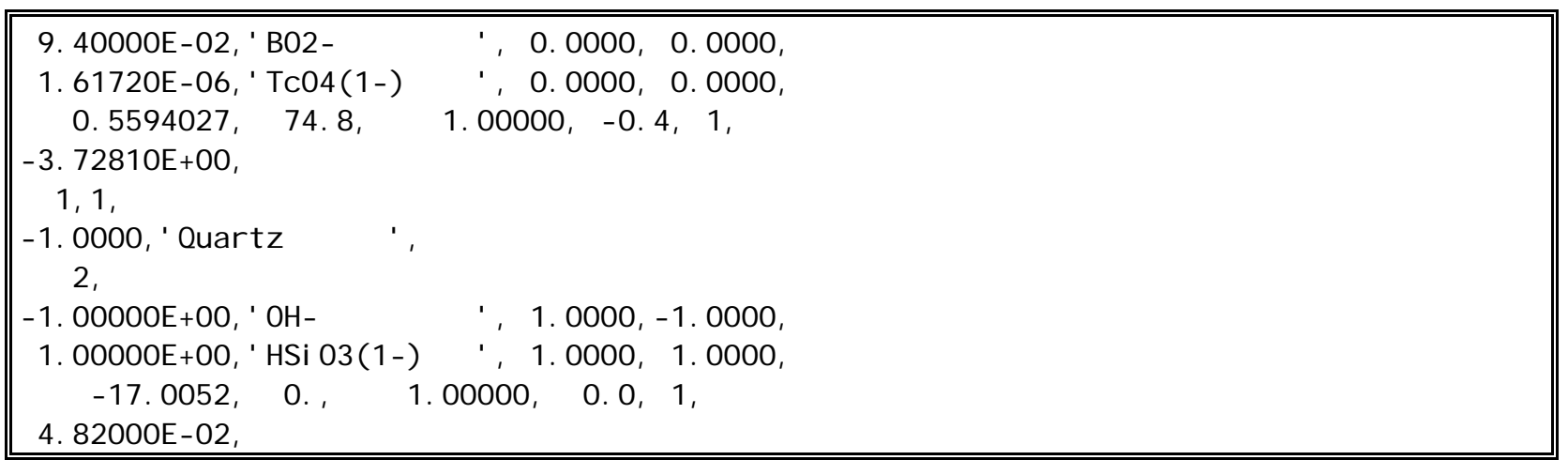

\section{A.27 Fluid Decay Card}

Card Title $^{\mathrm{a}}\{\sim$ Fluid Decay [ Card ] $\}$

Format: Char $^{a}$

Number of Fluid Decay Reactions ${ }^{\text {a }}$

Format: Integer ${ }^{\mathrm{a}}$

For: Number of Fluid Decay Reactions

Endfor:

Endcard:

\section{A.27.1 Fluid Decay Card Example}

$\sim$ FI uid Decay card

0 ,

\section{A.28 Solid Decay Card}

Card Title $^{\mathrm{a}}\{\sim$ Solid Decay [ Card ] $\}$

Format: Char $^{a}$

Number of Solid Decay Reactions ${ }^{\text {a }}$

Format: Integer ${ }^{\mathrm{a}}$

For: Number of Solid Decay Reactions

Endfor:

Endcard: 


\section{A.28.1 Solid Decay Card Example}

$\sim$ Sol id Decay card

0 ,

\section{A.29 Lithology Card}

Card Title $\{\sim$ Lithology [ Card ] $\}$

Format: Char $^{a}$

For: Number of Rock/Soil Types

Rock/Soil Name ${ }^{\mathrm{a}}$, Number of Solid Species ${ }^{\mathrm{b}}$,

Format: Char ${ }^{\mathrm{a}}$, Integer ${ }^{\mathrm{b}}$,

For: Number of Solid Species

Name of Solid Species ${ }^{a}$, Relative Solid Volume ${ }^{b}$, Specific Surface Area ${ }^{c}$

Format: Char $^{\mathrm{a}}, \mathrm{Real}^{\mathrm{b}}$, Real ${ }^{\mathrm{c}}$

Endfor:

Endfor:

Endcard:

\section{A.29.1 Lithology Card Example}

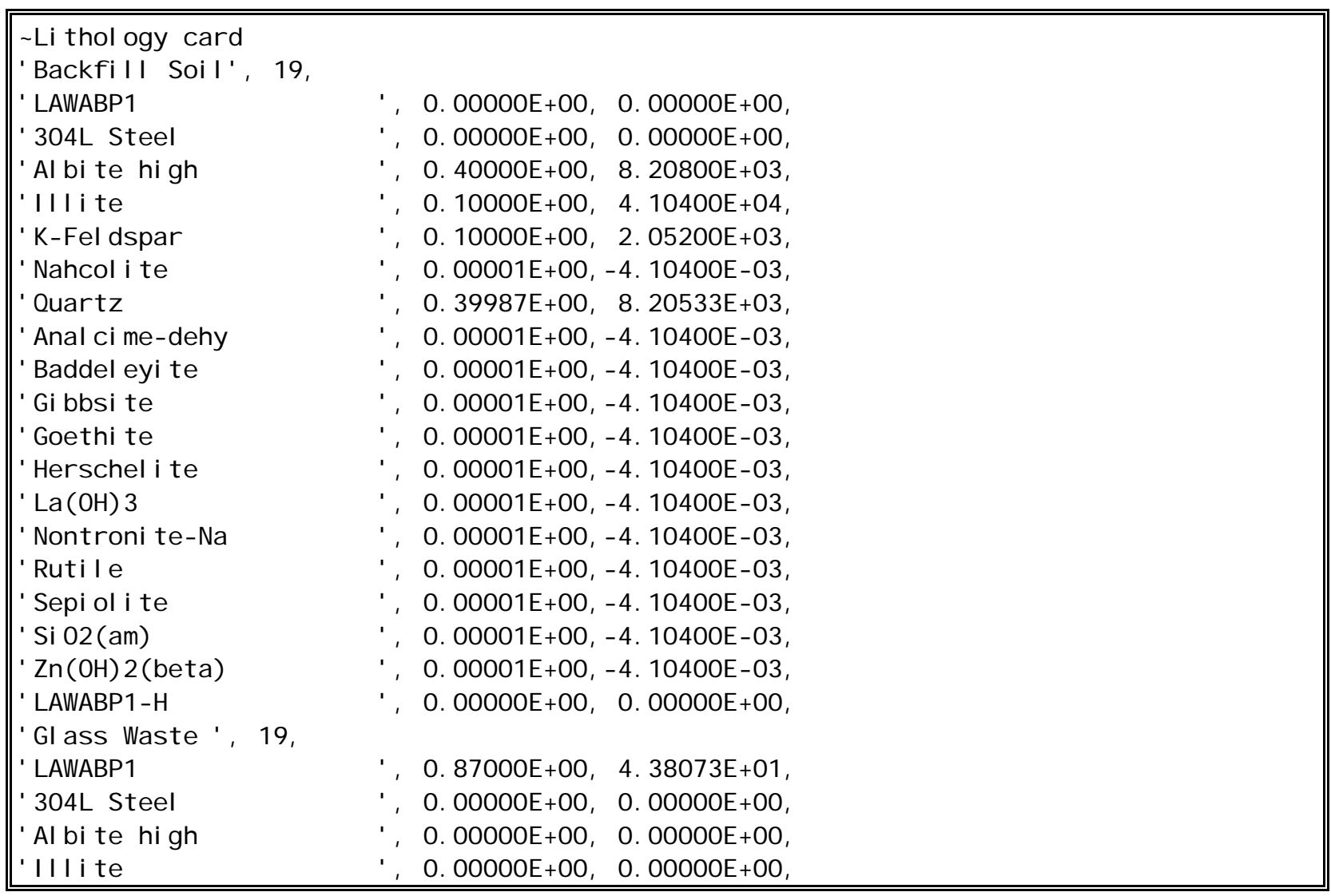




\begin{tabular}{|c|c|}
\hline 'K-Feldspar & $\therefore \quad 0.00000 E+00, \quad 0.00000 E+00$, \\
\hline ' Nahcolite & ', $0.00001 \mathrm{E}+00,-5.88000 \mathrm{E}-03$, \\
\hline ' Quartz & $\therefore \quad 0.00000 E+00,0.00000 E+00$, \\
\hline 'Analci me-dehy & $\therefore \quad 0.00001 E+00,-5.88000 E-03$, \\
\hline 'Baddel eyite & ', $0.00001 \mathrm{E}+00,-5.88000 \mathrm{E}-03$, \\
\hline 'Gibbsite & ' $\quad 0.00001 E+00,-5.88000 E-03$, \\
\hline 'Goethite & $', 0.00001 E+00,-5.88000 E-03$, \\
\hline 'Herschelite & ', $0.00001 \mathrm{E}+00,-5.88000 \mathrm{E}-03$, \\
\hline ' $\mathrm{La}(\mathrm{OH}) 3$ & ' $\quad 0.00001 E+00,-5.88000 E-03$, \\
\hline 'Nontronite- $\mathrm{Na}$ & $', 0.00001 E+00,-5.88000 E-03$, \\
\hline 'Rutile & $', 0.00001 E+00,-5.88000 E-03$, \\
\hline 'Sepiolite & $', 0.00001 E+00,-5.88000 E-03$, \\
\hline ' Si 02 ( a m) & $', 0.00001 E+00,-5.88000 E-03$, \\
\hline ' $\mathrm{Zn}(\mathrm{OH}) 2$ (bet a) & $', 0.00001 E+00,-5.88000 E-03$, \\
\hline ' LAWABP 1 - H & $\therefore, 0.00001 \mathrm{E}+00, \quad 5.03532 \mathrm{E}-04$, \\
\hline
\end{tabular}

\section{A.30 Reaction BC Card}

Card Title $^{\mathrm{a}}\{\sim$ Reaction BC [ Card ] $\}$

Format: Char $^{a}$

Number of Boundary Segments ${ }^{\text {a }}$

Format: Integer ${ }^{\mathrm{a}}$

For: Number of Boundary Segments

Boundary Segment Number ${ }^{\mathrm{a}}$, Boundary Condition Type ${ }^{\mathrm{b}}\{1=$ Diriclet $\}$

Format: Integer ${ }^{\mathrm{a}}$, Integer ${ }^{\mathrm{b}}$

Number of Solids in Equilibrium with Solution,

Format: Integer ${ }^{\mathrm{a}}$,

For: Number of Solids in Equilibrium with Solution,

Reaction Number of Solid Reaction ${ }^{\mathrm{a}}$

Format: Integer ${ }^{\mathrm{a}}$,

Endfor:

Number of Kinetic Reactions in Equilibrium with Solution ${ }^{\mathrm{a}}$,

Format: Char, Integer ${ }^{\mathrm{a}}$,

For: Number of Kinetic Reactions in Equilibrium with Solution,

Reaction Number of Kinetic Reaction ${ }^{\text {a }}$

Format: Integer ${ }^{\mathrm{a}}$,

\section{Endfor:}

Number of Gases in Equilibrium with Solution ${ }^{\mathrm{a}}$,

Format: Integer ${ }^{\mathrm{a}}$,

For: Number of Gases in Equilibrium with Solution,

Gas name $^{\mathrm{a}}$, Gas partial pressure (atm) ${ }^{\mathrm{b}}$

Format: $\operatorname{Char}^{a}$, Real ${ }^{\mathrm{b}}$,

\section{Endfor:}


Number of Specified Aqueous Species Concentrations ${ }^{\mathrm{a}}$

Format: Integer ${ }^{\mathrm{a}}$,

For: Number of Specified Aqueous Species Concentrations,

Specifiec Total Concentration ${ }^{a}$, Number of Aqueous Species ${ }^{b}$

Format: Real ${ }^{\mathrm{a}}$, Integer ${ }^{\mathrm{b}}$

Stoichiometric Coefficient of Aquous Species ${ }^{\mathrm{a}}$, Name of Aqueous Species ${ }^{\mathrm{b}}$

Format: Real $^{\mathrm{a}}$, Char ${ }^{\mathrm{a}}$,

Endfor:

\section{Endfor:}

\section{Endcard:}

\section{A.30.1 Reaction BC Card Example}

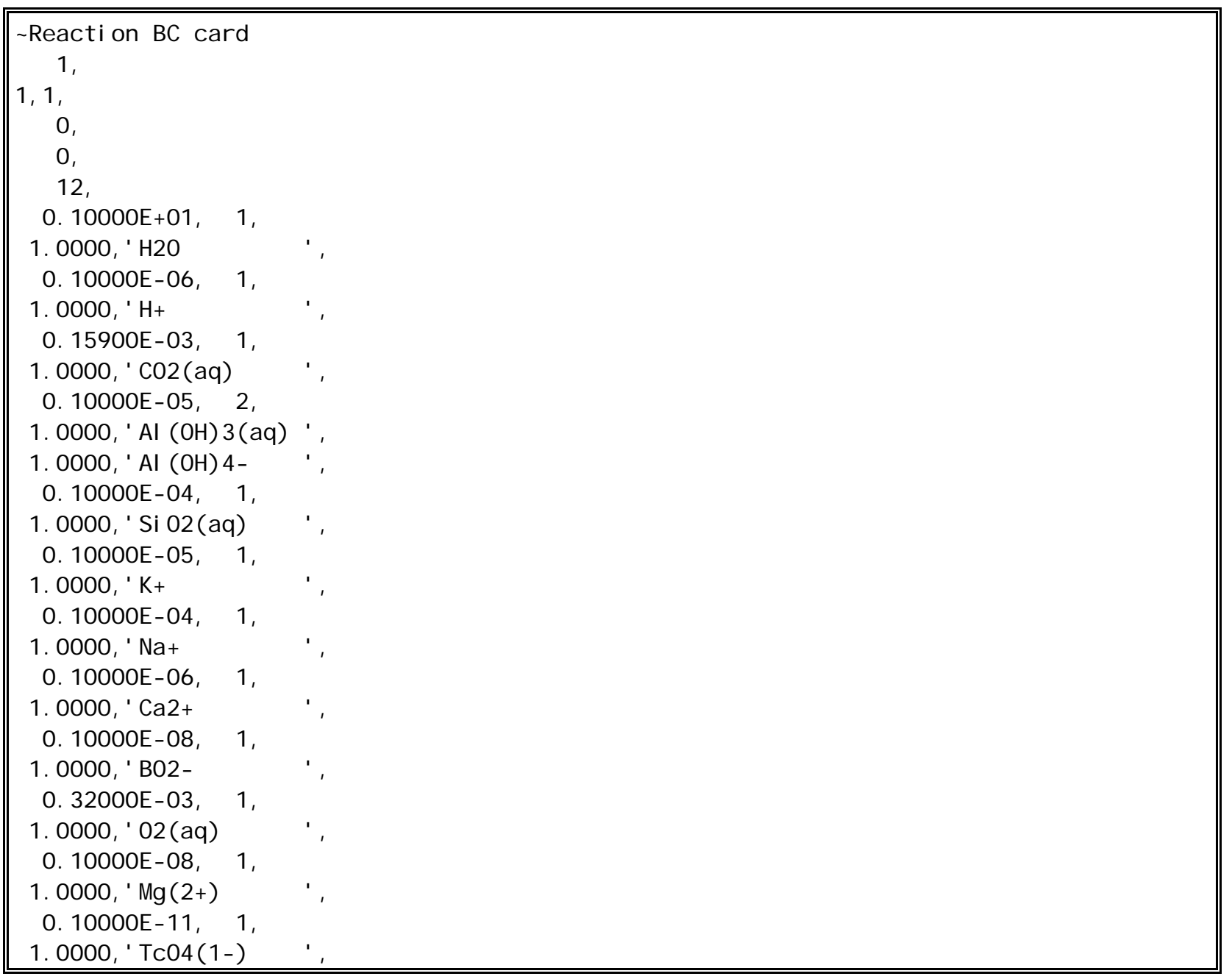




\section{A.31 Reaction IC card}

Card Title $^{\mathrm{a}}\{\sim$ Reaction IC [ Card ] $\}$

Format: Char $^{a}$

Number of Reaction Initial Conditions ${ }^{a}$

Format: Integer ${ }^{\mathrm{a}}$

For: Number of Reaction Initial Conditions

Initial Condition Zone Number ${ }^{\mathrm{a}}$, Boundary Condition Type ${ }^{\mathrm{b}}\{1=$ Uniform $\}$

Format: Integer ${ }^{\mathrm{a}}$, Integer ${ }^{\mathrm{b}}$

Number of Solids in Equilibrium with Solution ${ }^{\mathrm{a}}$,

Format: Integer ${ }^{\mathrm{a}}$,

For: Number of Solids in Equilibrium with Solution,

Reaction Number of Solid Reaction ${ }^{\mathrm{a}}$

Format: Integer ${ }^{\mathrm{a}}$,

Endfor:

Number of Kinetic Reactions in Equilibrium with Solution ${ }^{\mathrm{a}}$,

Format: Char, Integer ${ }^{\mathrm{a}}$,

For: Number of Kinetic Reactions in Equilibrium with Solution,

Reaction Number of Kinetic Reaction ${ }^{\text {a }}$

Format: Integer ${ }^{\mathrm{a}}$,

Endfor:

Number of Gases in Equilibrium with Solution ${ }^{\mathrm{a}}$,

Format: Integer ${ }^{\mathrm{a}}$,

For: Number of Gases in Equilibrium with Solution,

Gas name ${ }^{\mathrm{a}}$, Gas partial pressure (atm) ${ }^{\mathrm{b}}$

Format: Char $^{a}$, Real ${ }^{\mathrm{b}}$,

\section{Endfor:}

Number of Specified Aqueous Species Concentrations ${ }^{\mathrm{a}}$

Format: Integer ${ }^{\mathrm{a}}$,

For: Number of Specified Aqueous Species Concentrations,

Specifiec Total Concentration ${ }^{\mathrm{a}}$, Number of Aqueous Species ${ }^{\mathrm{b}}$

Format: Real ${ }^{\mathrm{a}}$, Integer ${ }^{\mathrm{b}}$

Stoichiometric Coefficient of Aquous Species ${ }^{\mathrm{a}}$, Name of Aqueous Species ${ }^{\mathrm{b}}$

Format: Real ${ }^{\mathrm{a}}$, Char ${ }^{\mathrm{a}}$,

Endfor:

Endfor:

Endcard: 


\section{A.31.1 Reaction IC Card Example}

Reaction I C card

0 ,

Note: the default is for the initial conditions to be equal to the inlet boundary conditions; otherwise input is identical to Reaction BC Card. 
Appendix B

STORM Version 3.0 UNIX Scripts 


\section{Appendix B \\ STORM Version 3.0 UNIX Scripts}

\section{B.1 Makefile}

Example makefile for compiling parallel STORM under the Linux operating system with the Intel compiler

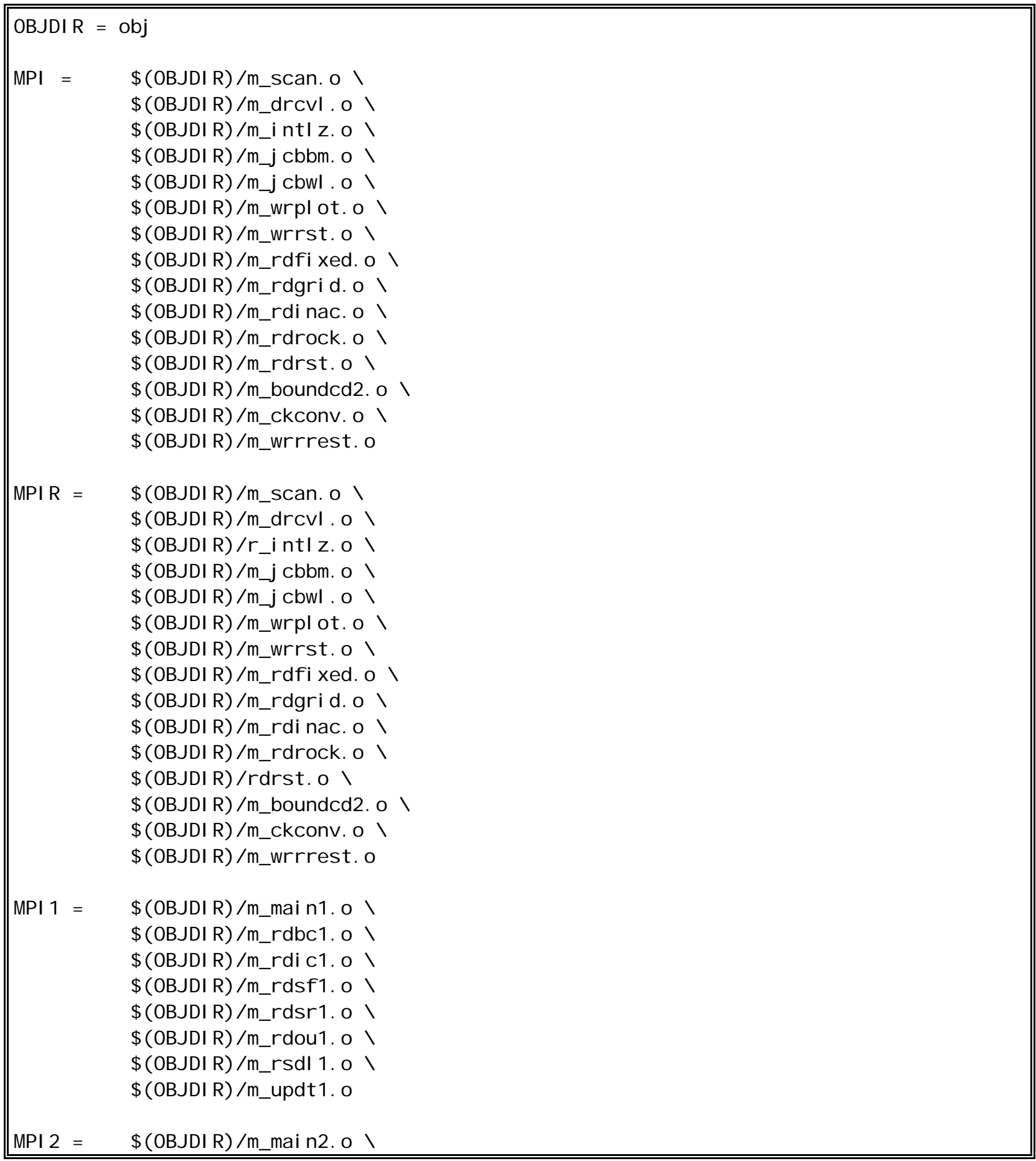

B. 1 


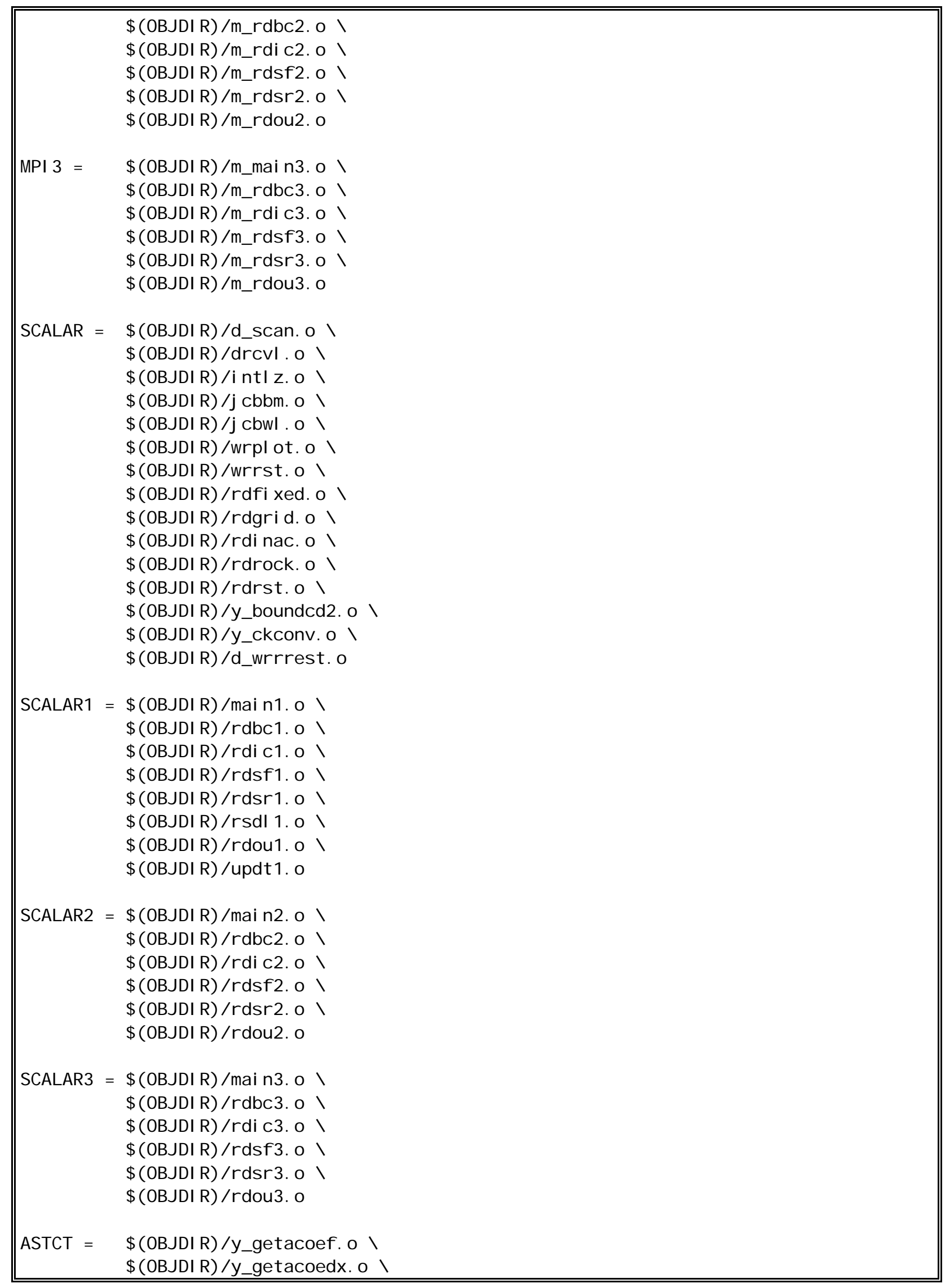

B. 2 


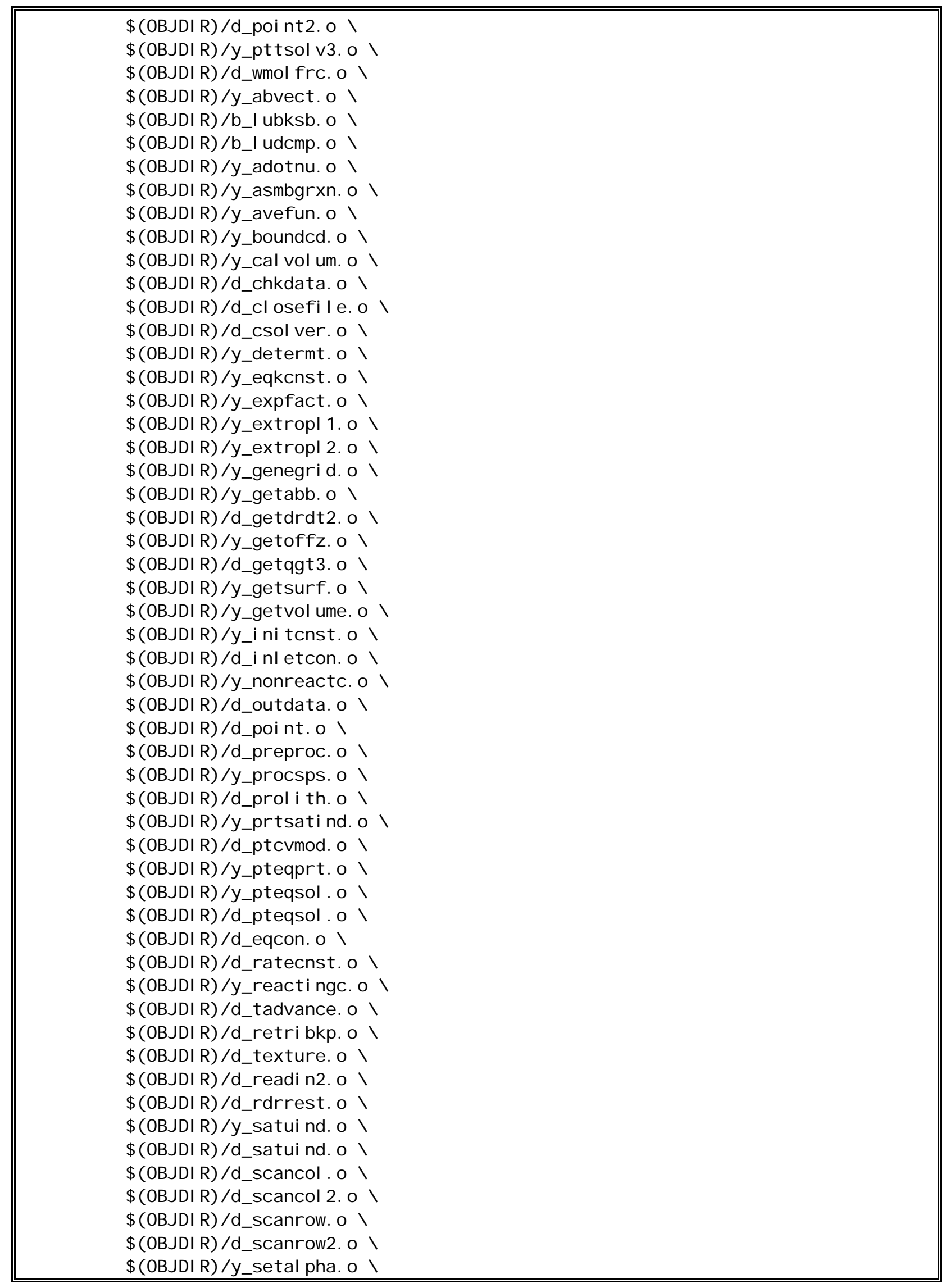

B. 3 


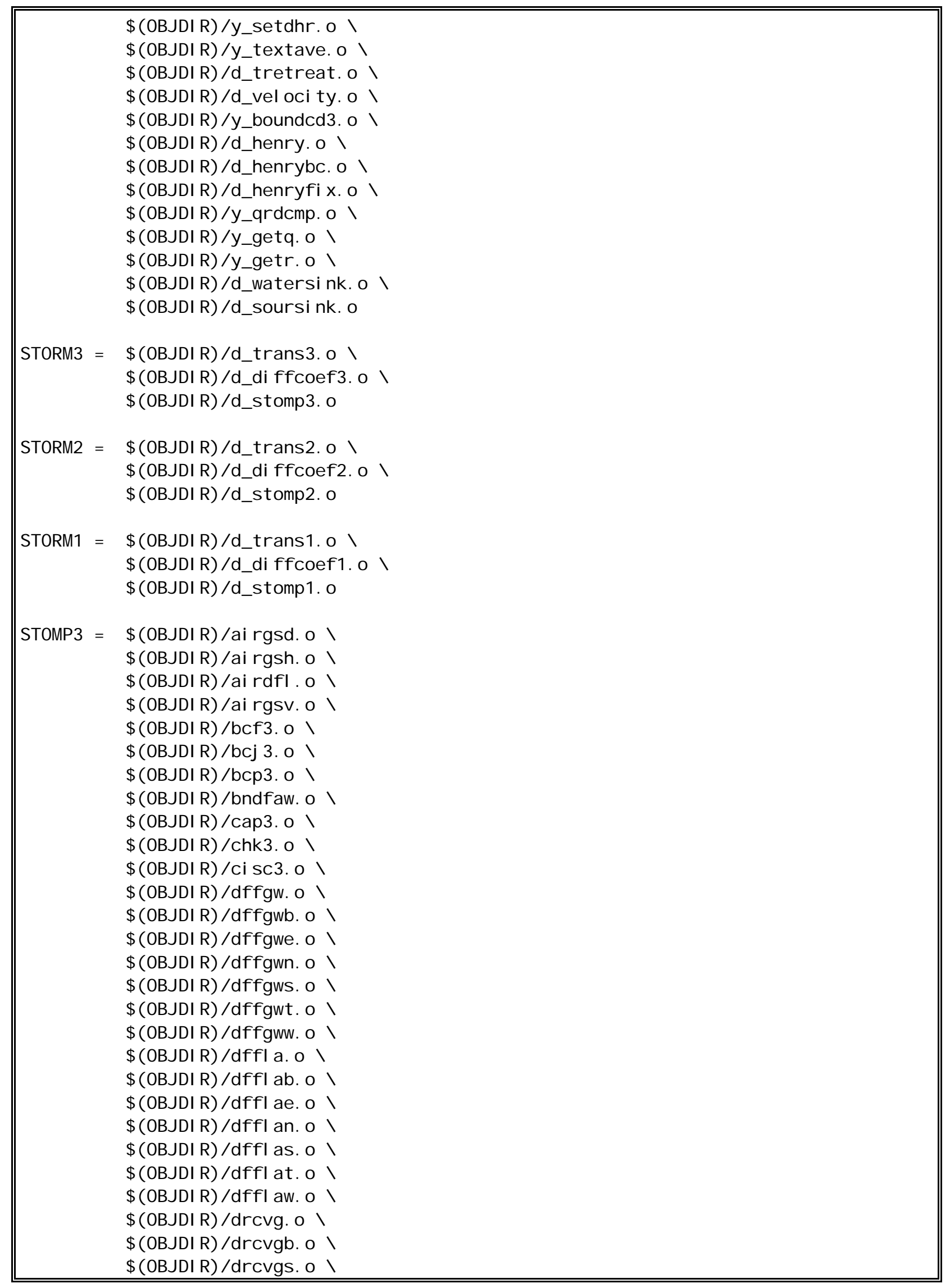

B. 4 


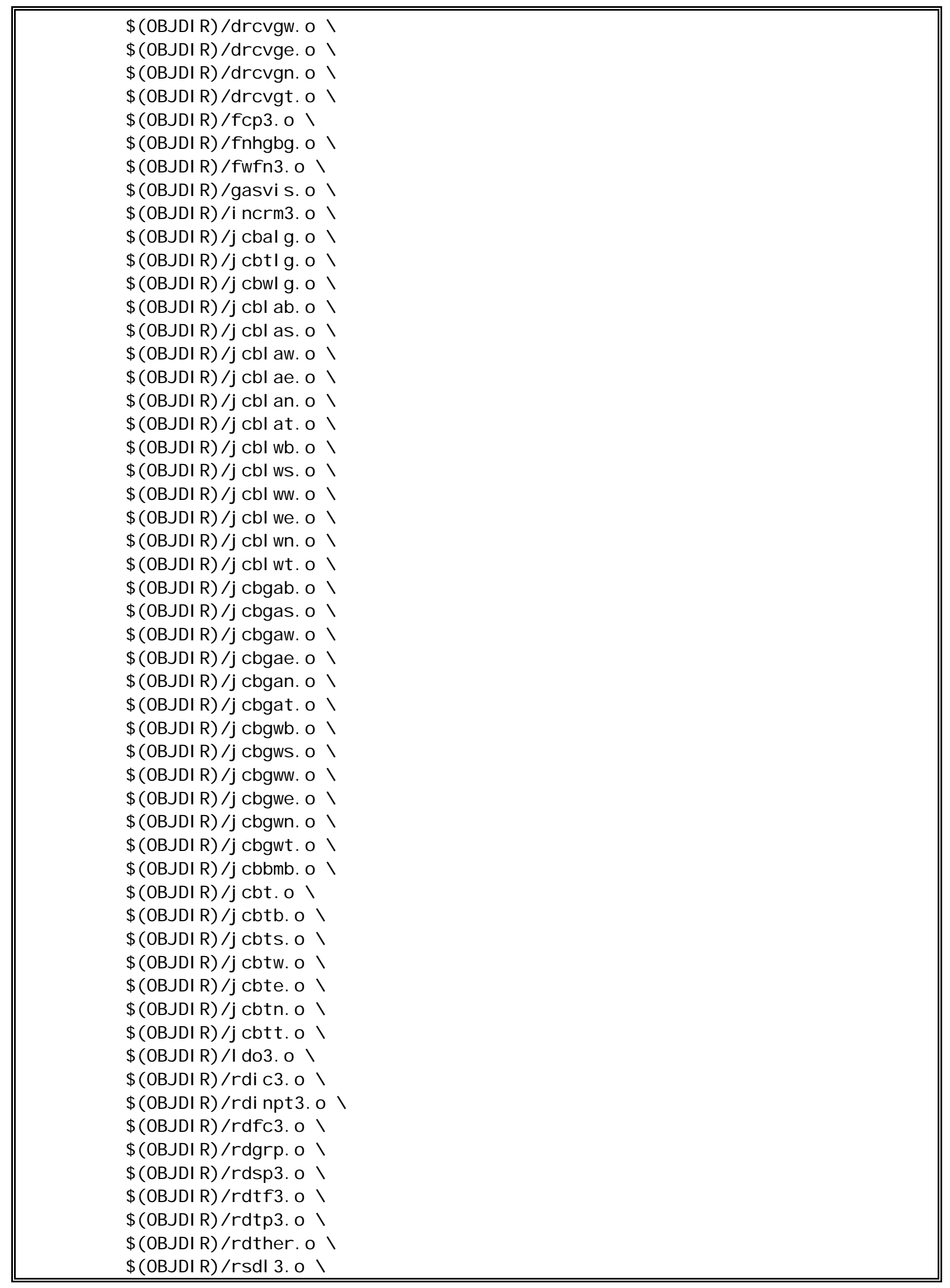

B. 5 


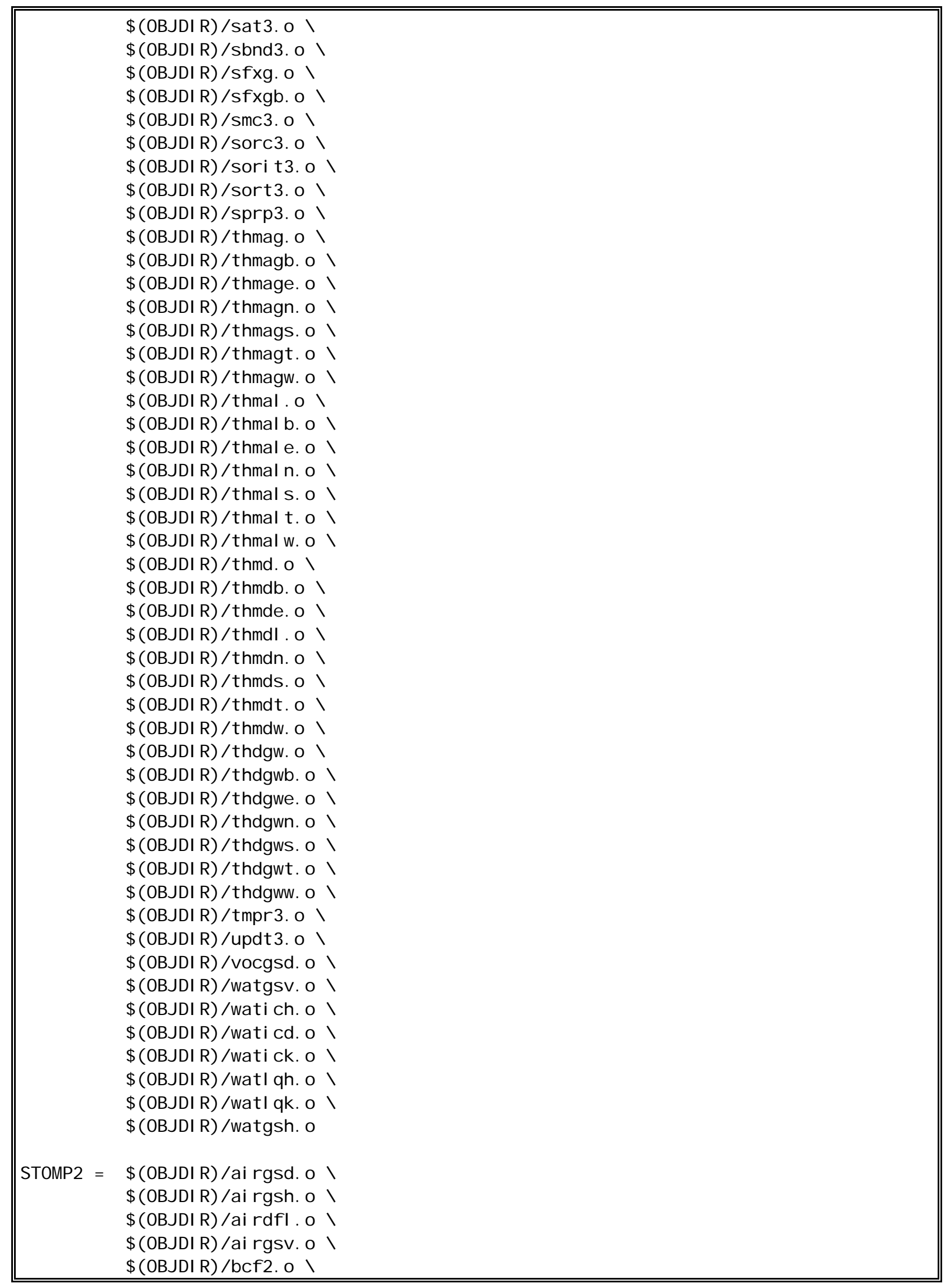




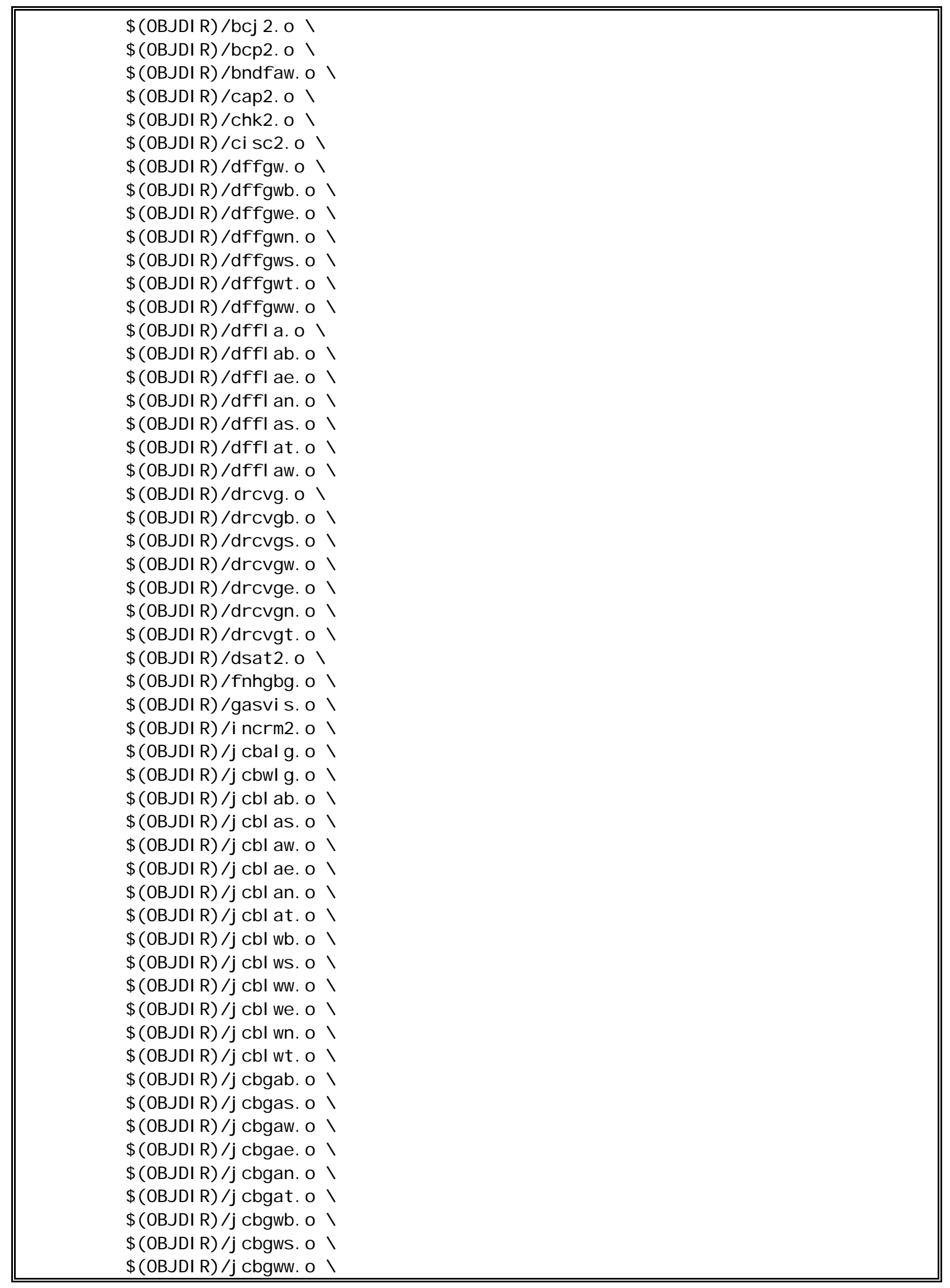

B. 7 


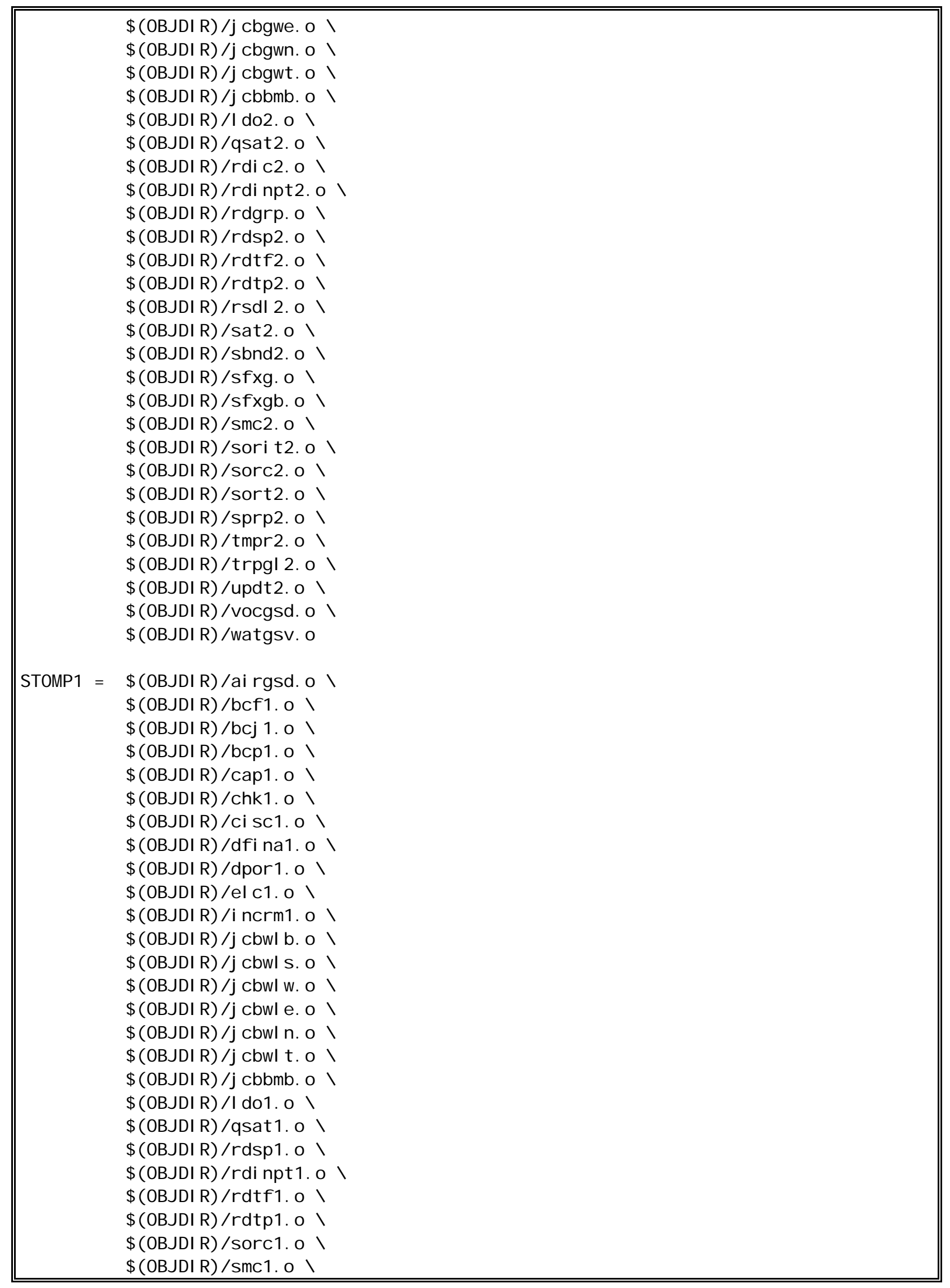

B. 8 


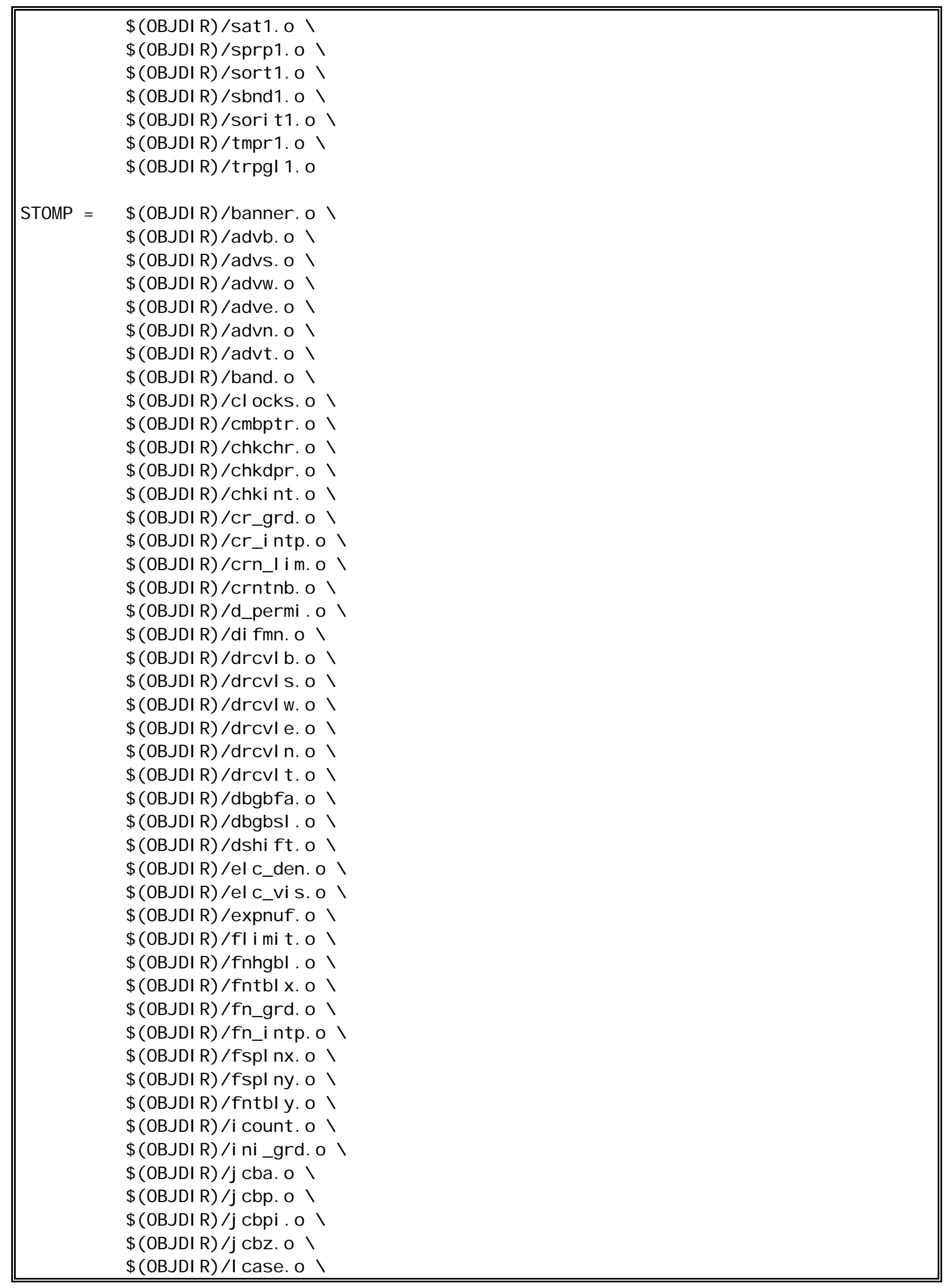

B. 9 


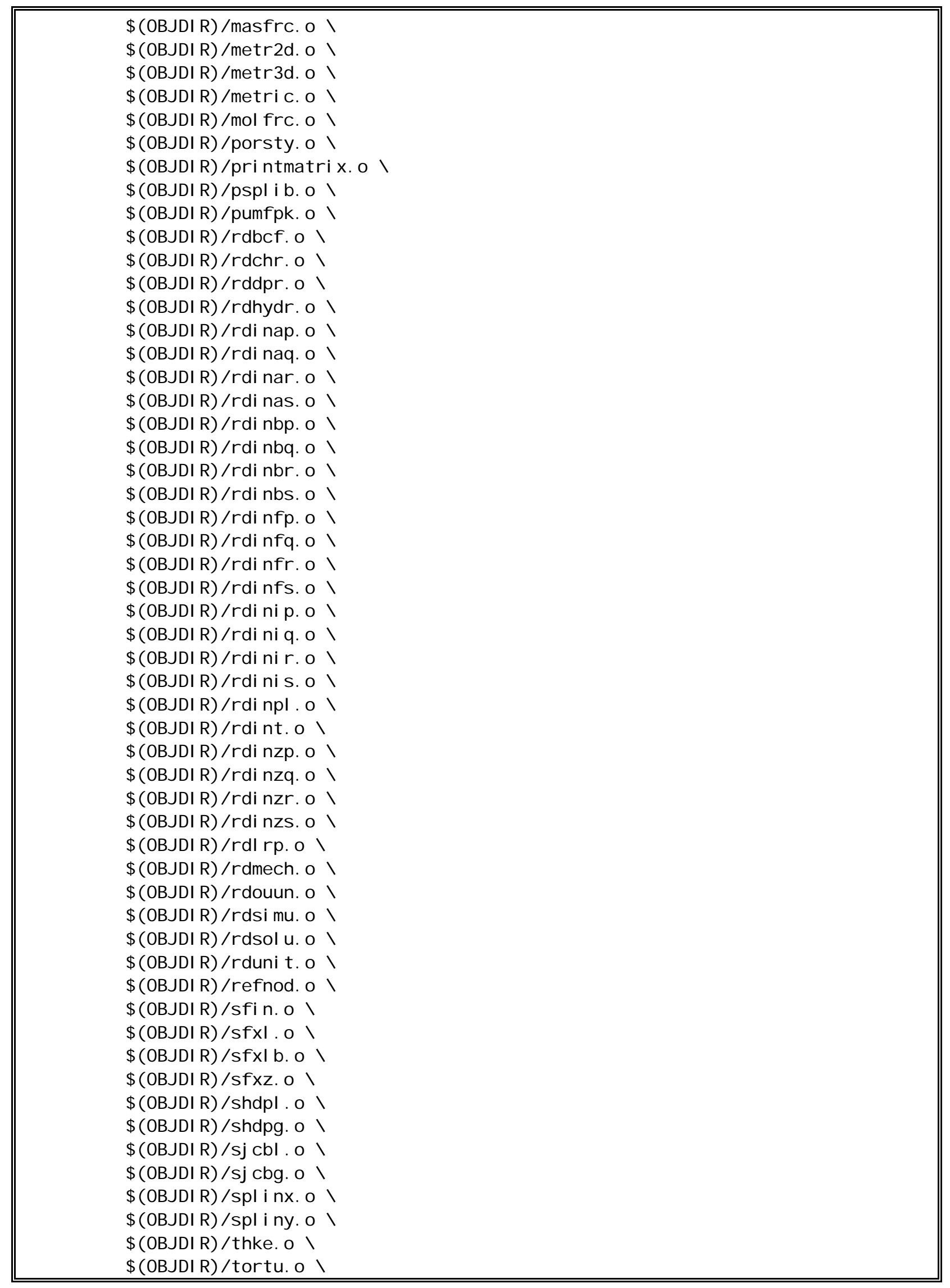

B. 10 


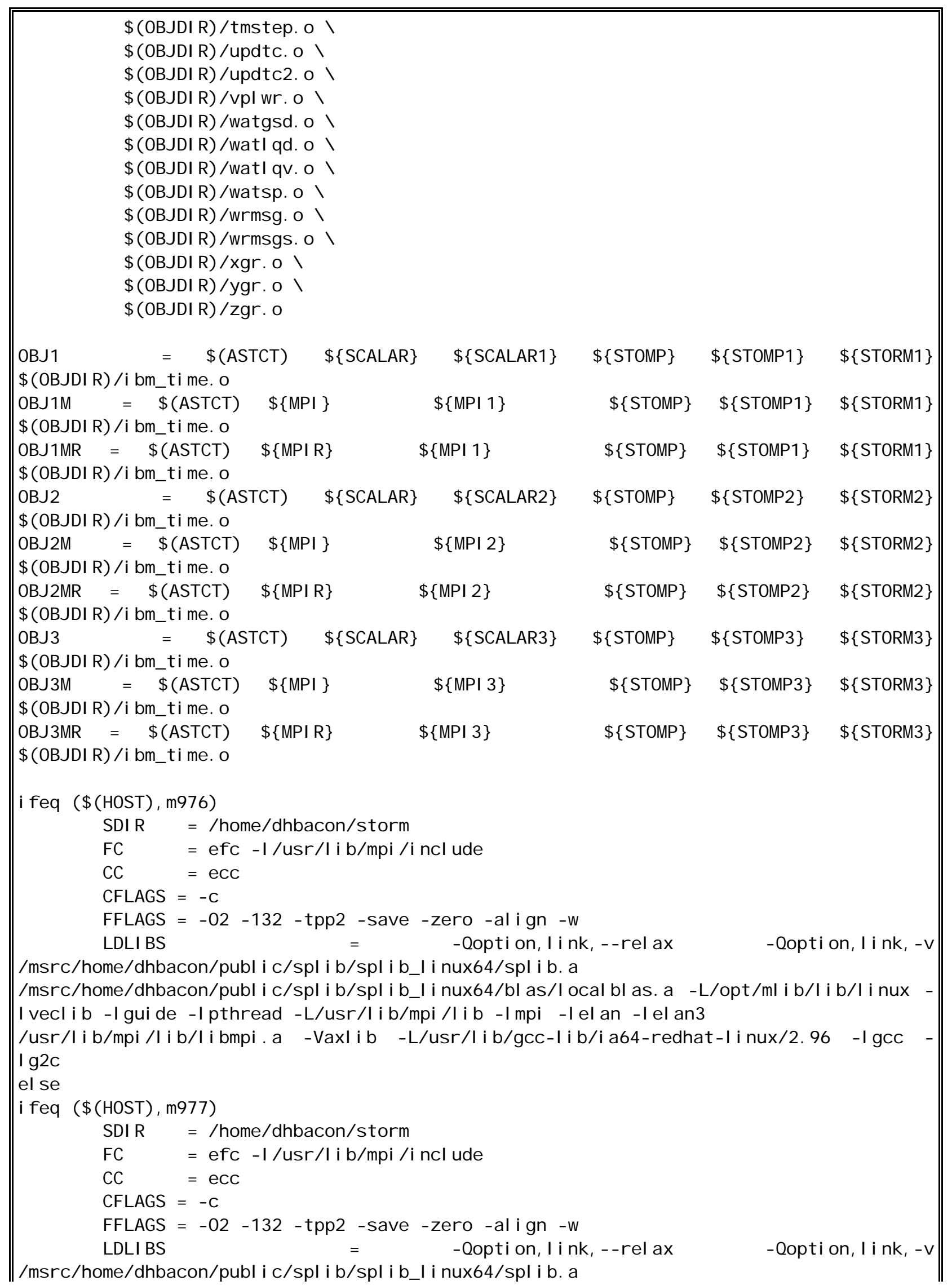

B.11 


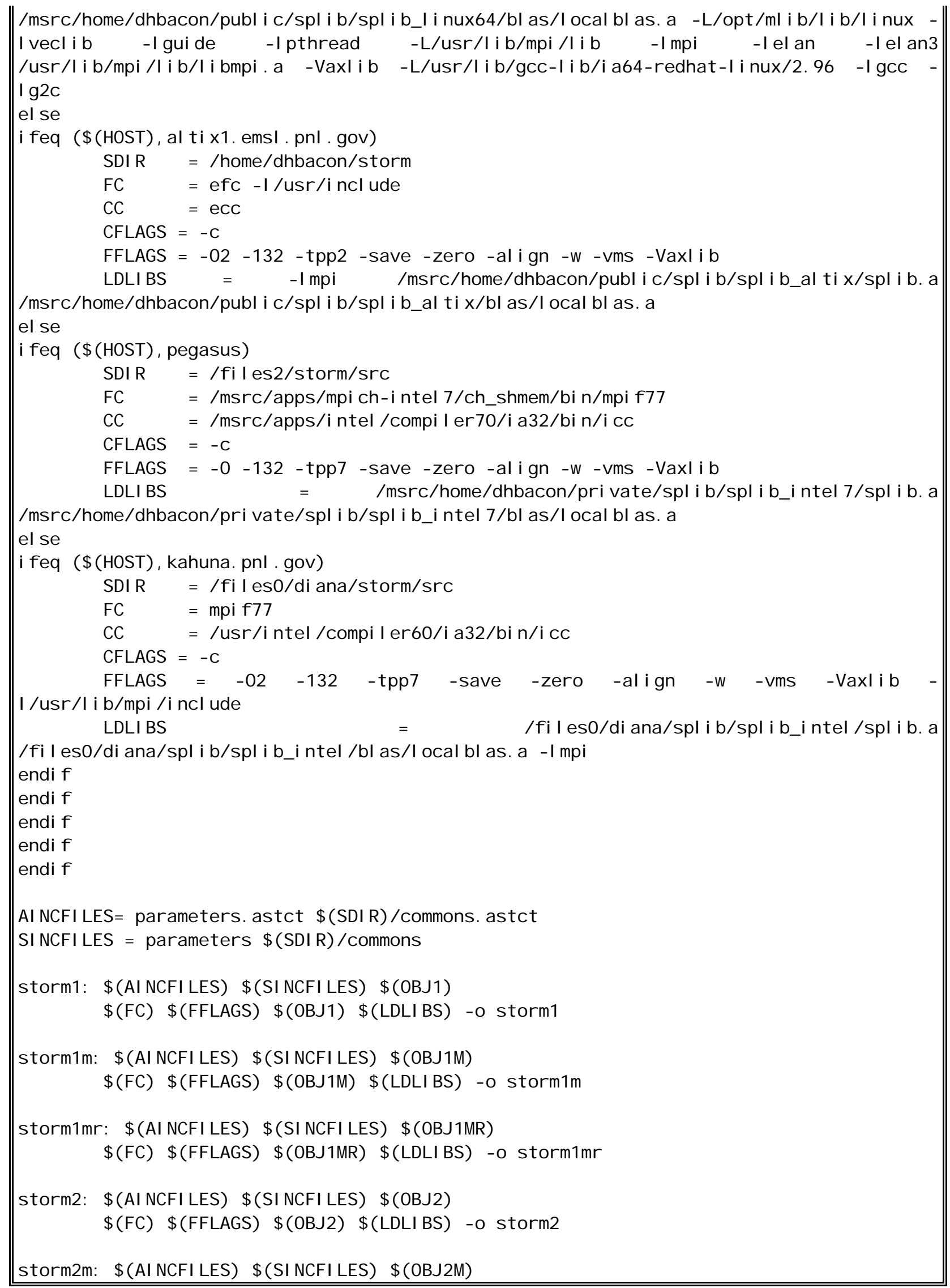

B. 12 


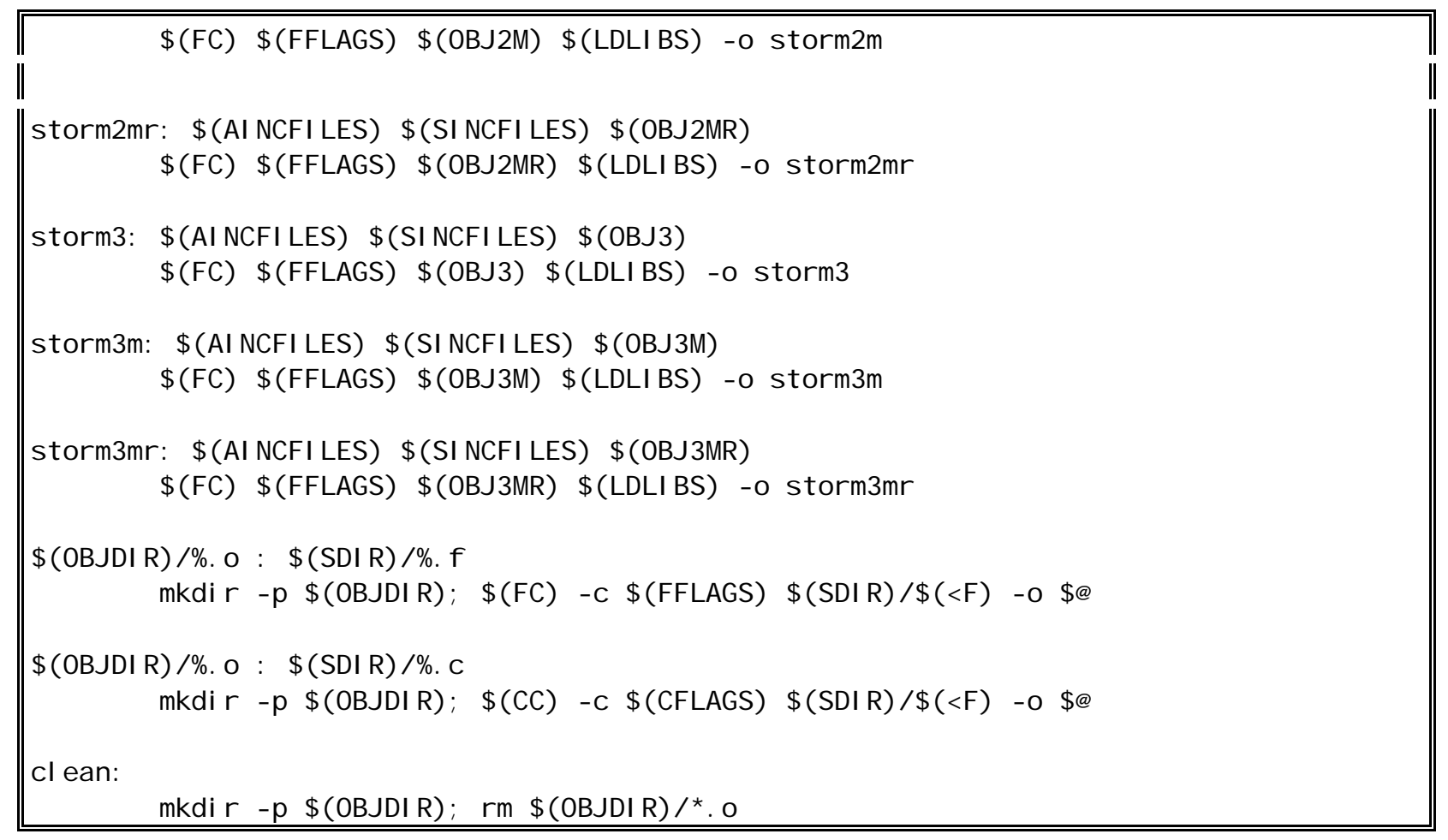

\section{B.2 Run Script}

Example script for running parallel STORM on mpp2

\begin{tabular}{|c|c|c|c|}
\hline \multicolumn{4}{|c|}{ \#BSUB - P ab1234 } \\
\hline \#BSUB & $-n 64$ & & \\
\hline \#BSUB & -W 24:00 & & \\
\hline \#BS UB & - J storml m_bulkvit_refractory_ $09_{-} 8 \times 8 p$ & & \\
\hline \#BS UB & - e error_ $0 . \%]$ & & \\
\hline \#BS UB & - 0 screen_ $0 . \%$ & & \\
\hline \#BSUB & - udiana.bacon@pnl.gov & & \\
\hline \#BS UB & $-\mathrm{N}$ & & \\
\hline prun & $-v$ & 64 & st or ml m \\
\hline
\end{tabular}




\section{Appendix C}

\section{Data Files}




\section{Appendix C \\ Data Files}

\section{C.1 Input File for Aqueous Phase Advection and Diffusion Test Case}

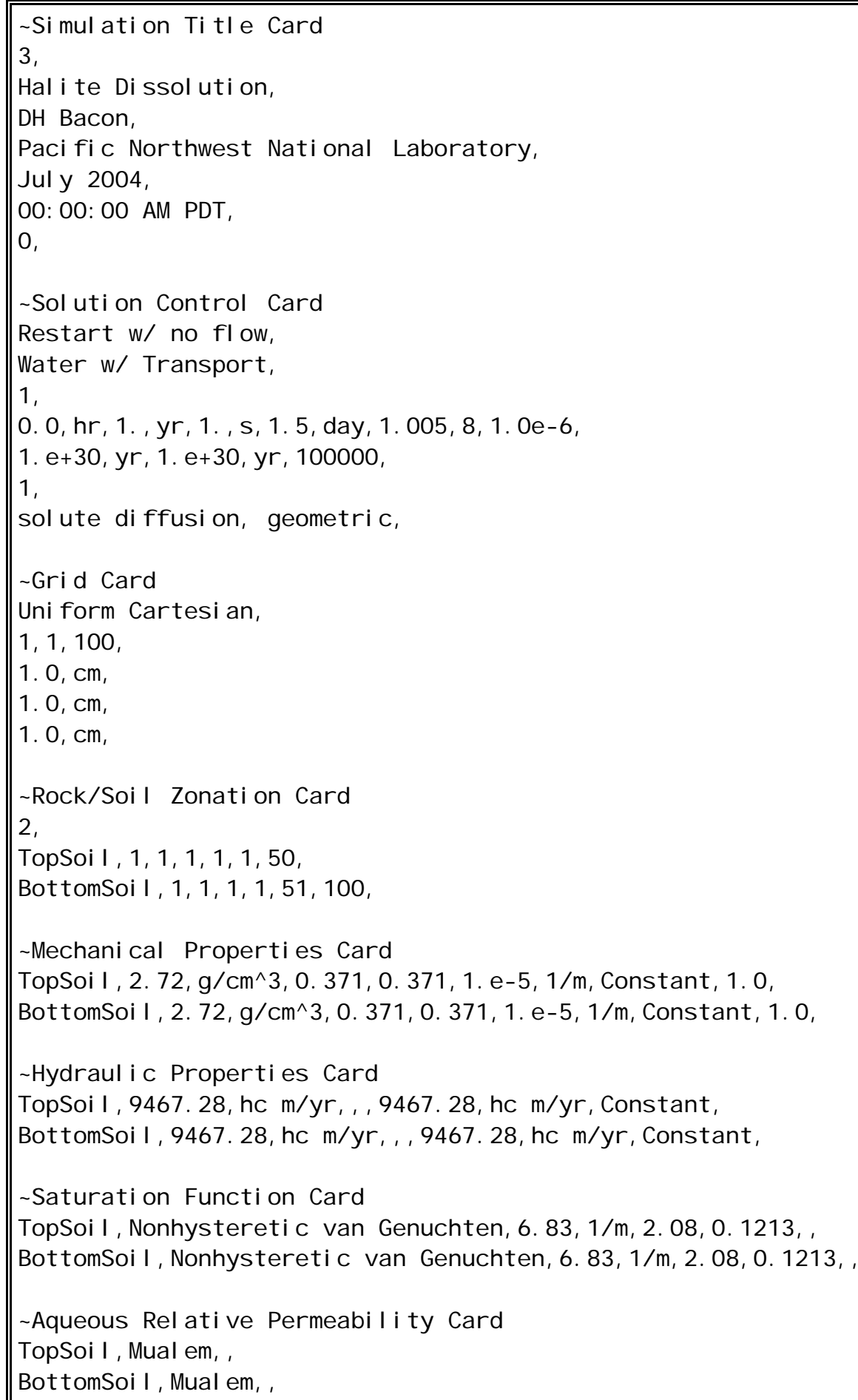




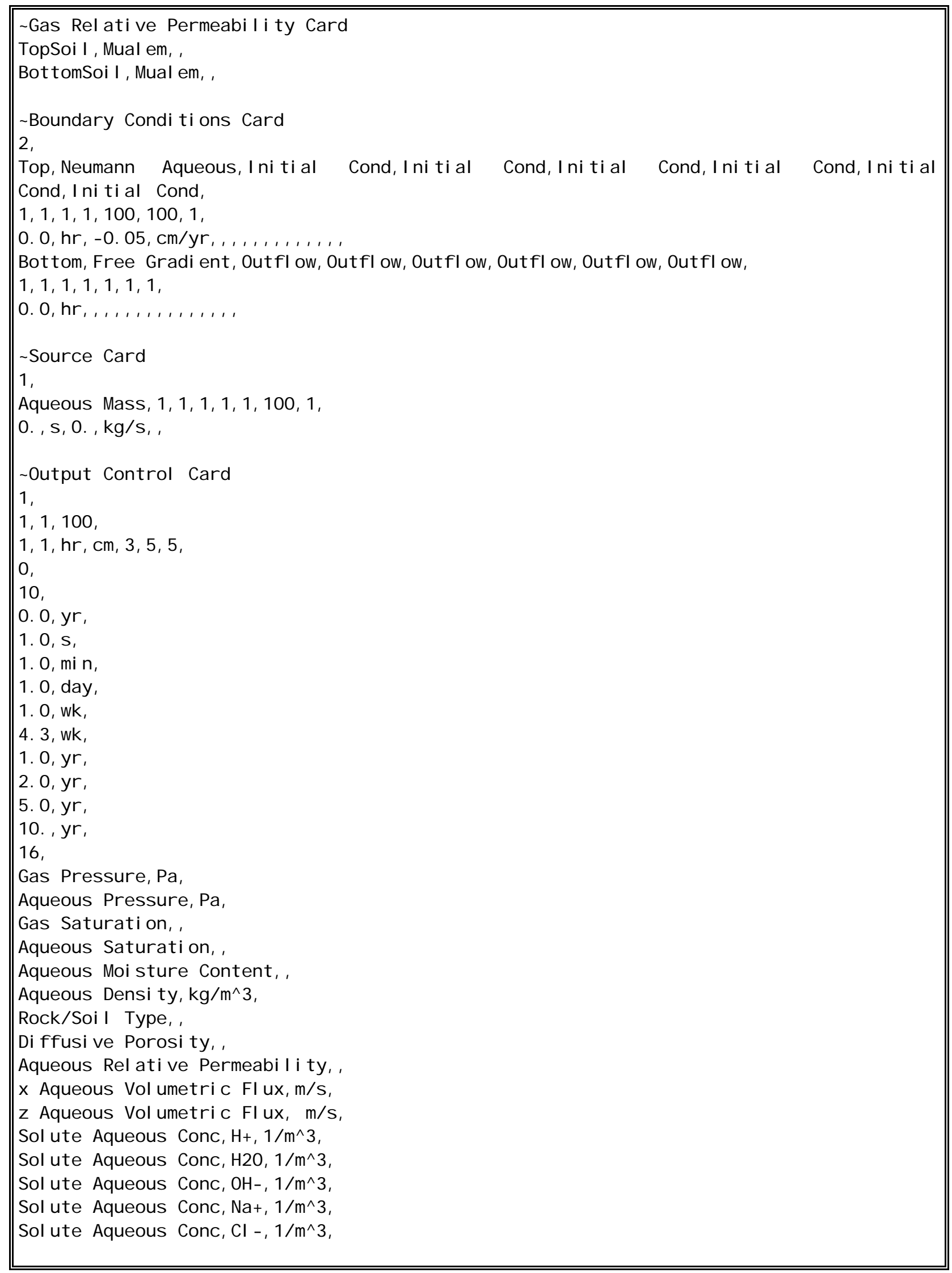




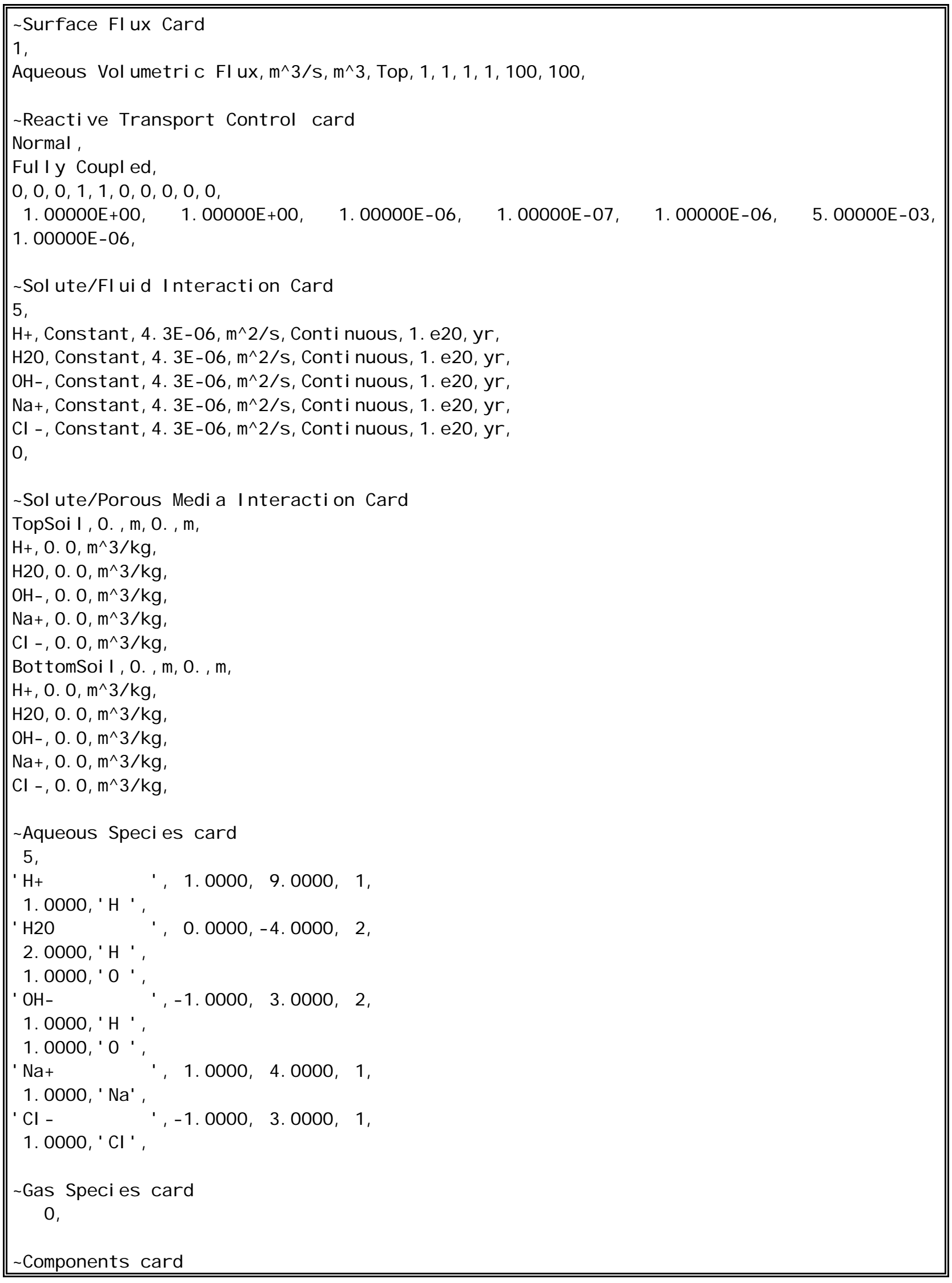




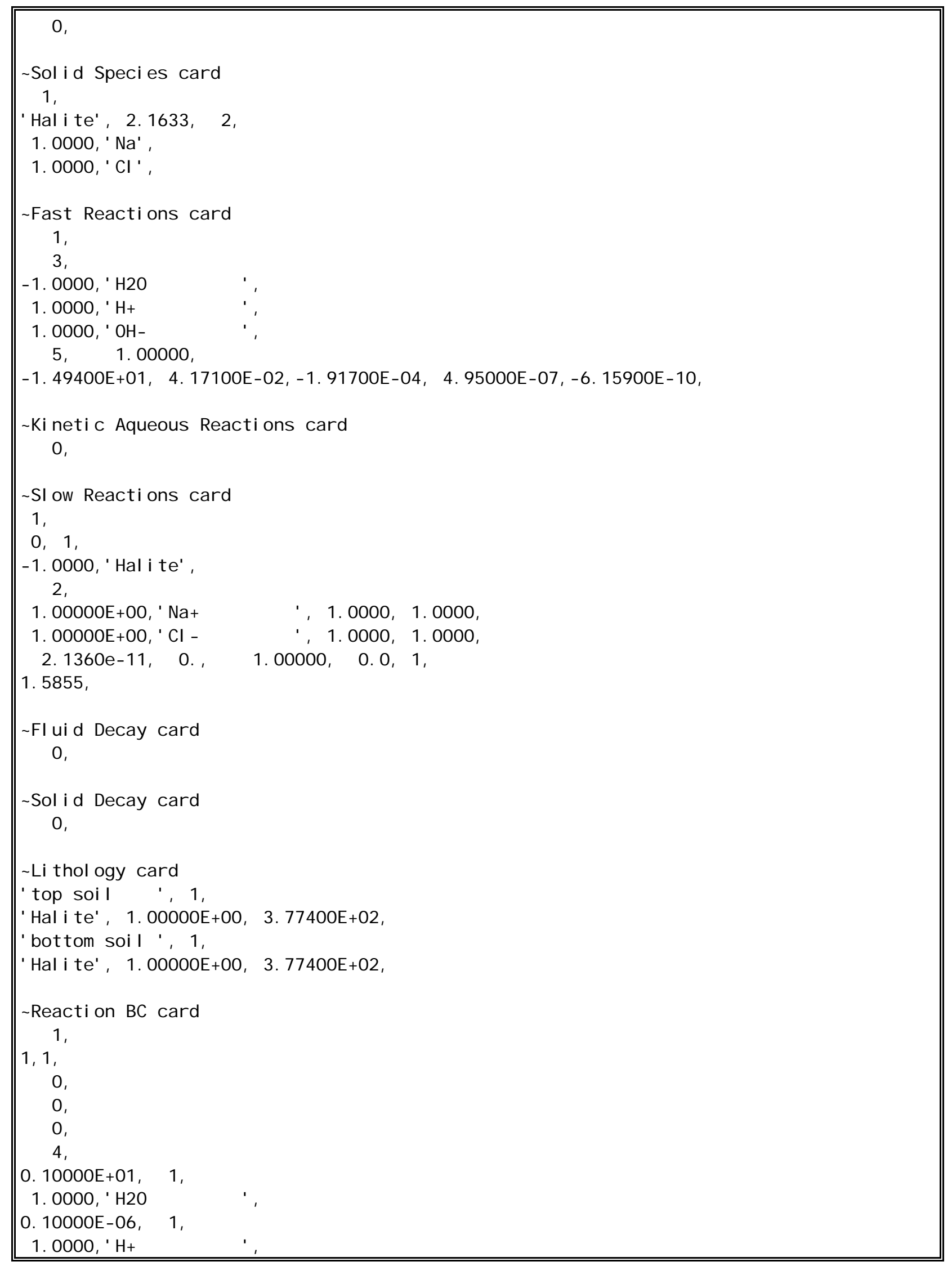




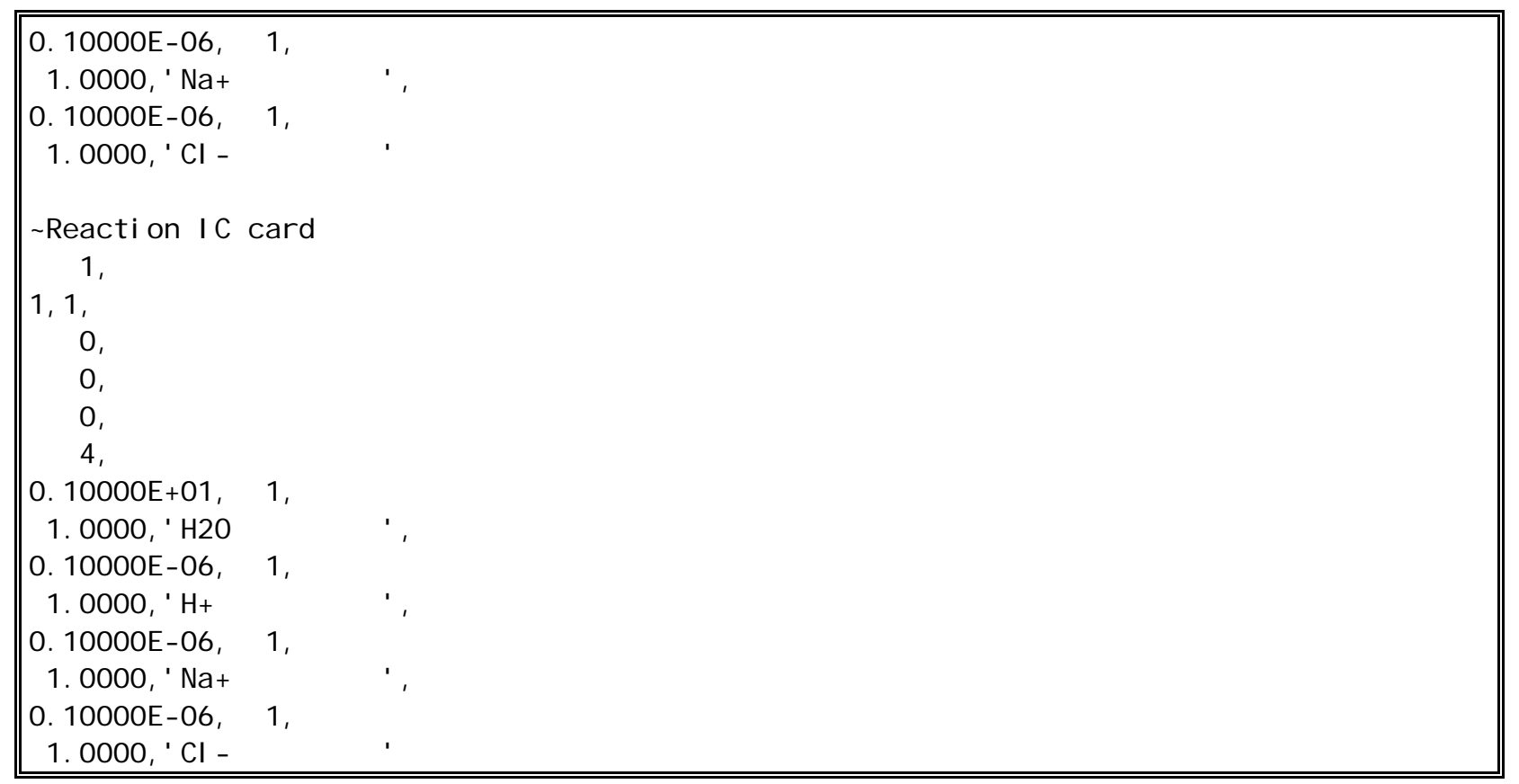




\section{C.2 STORM Input Data File for Gas Phase Advection/Diffusion Test Case}

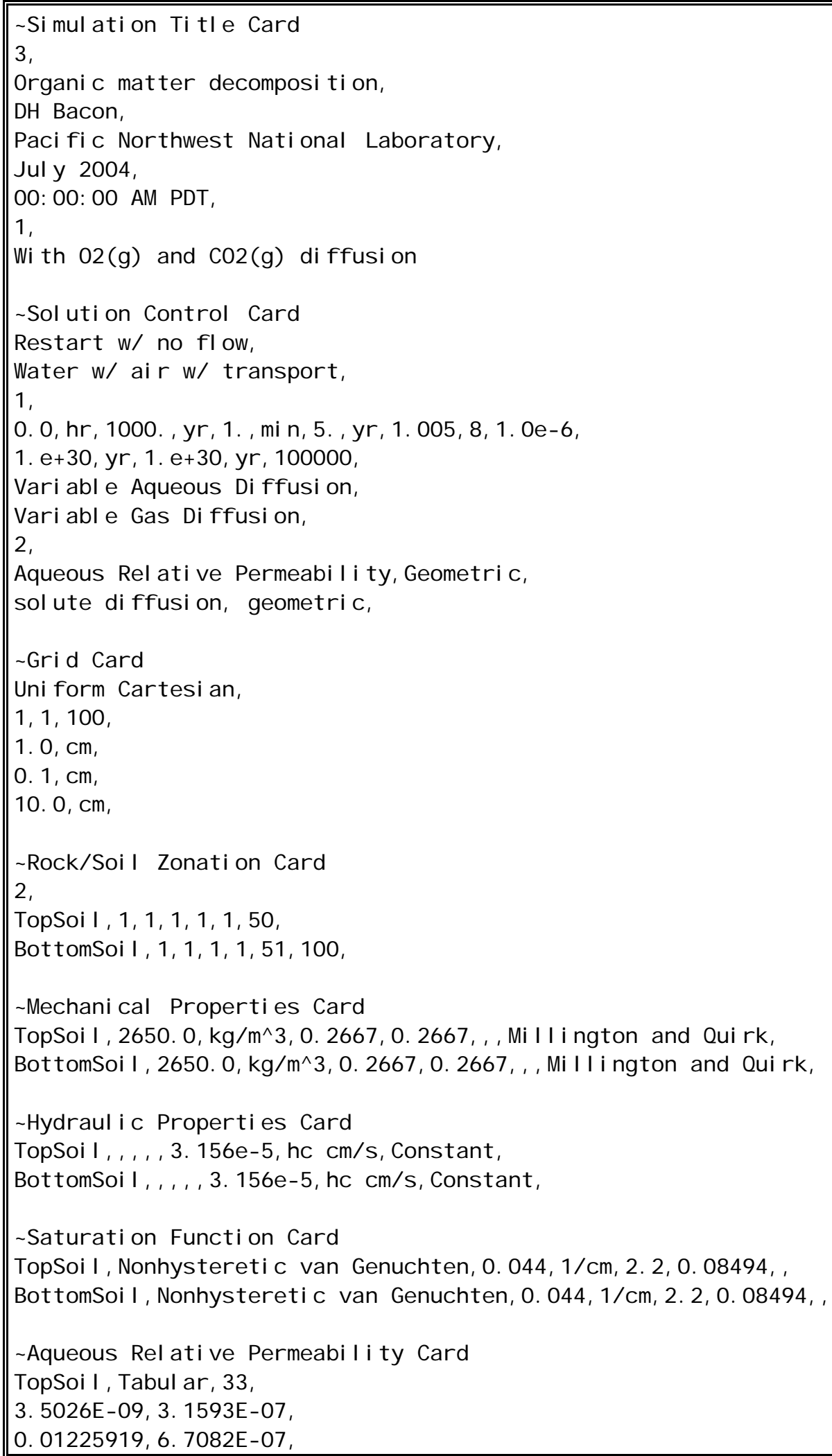




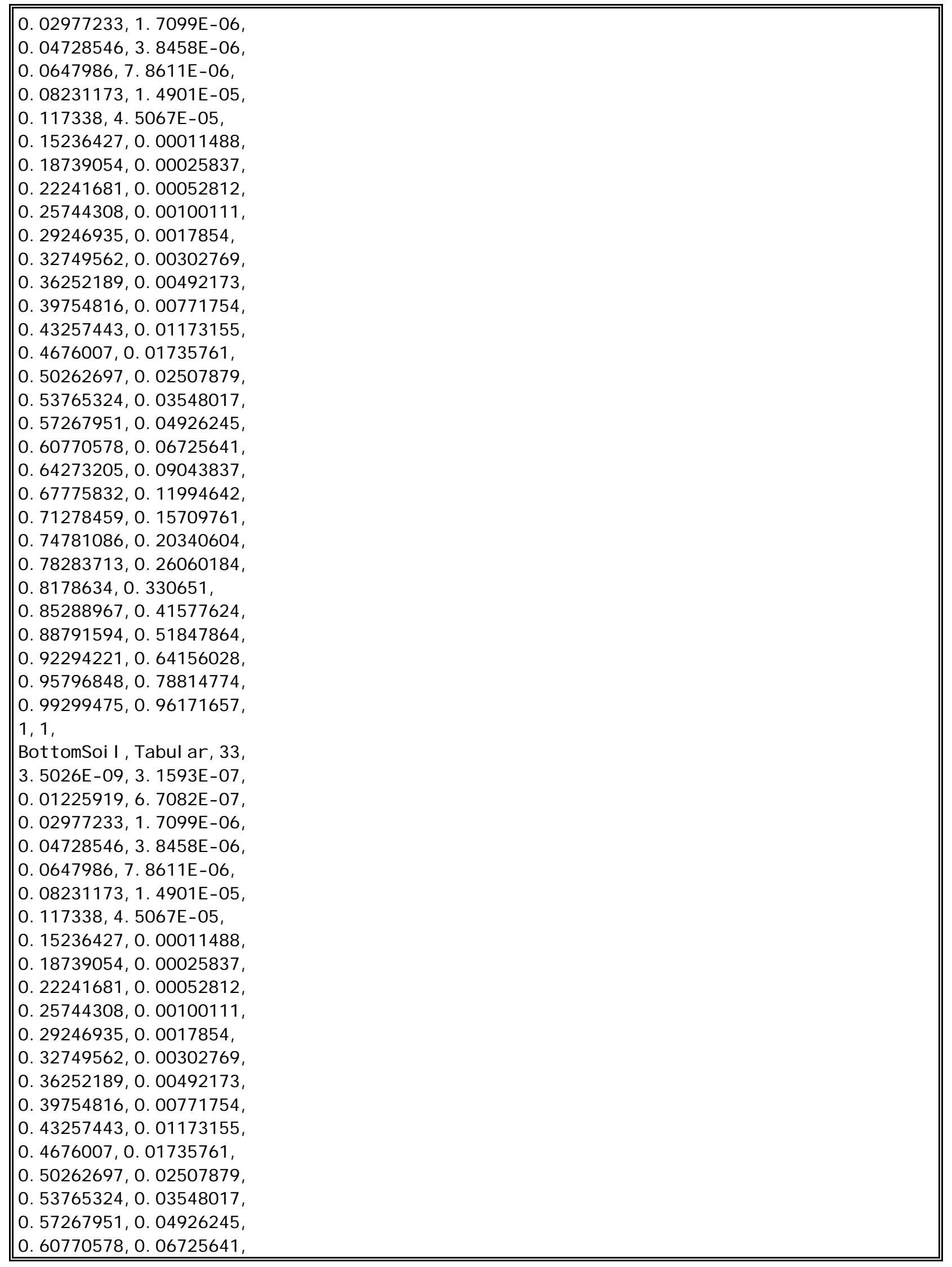

C. 7 


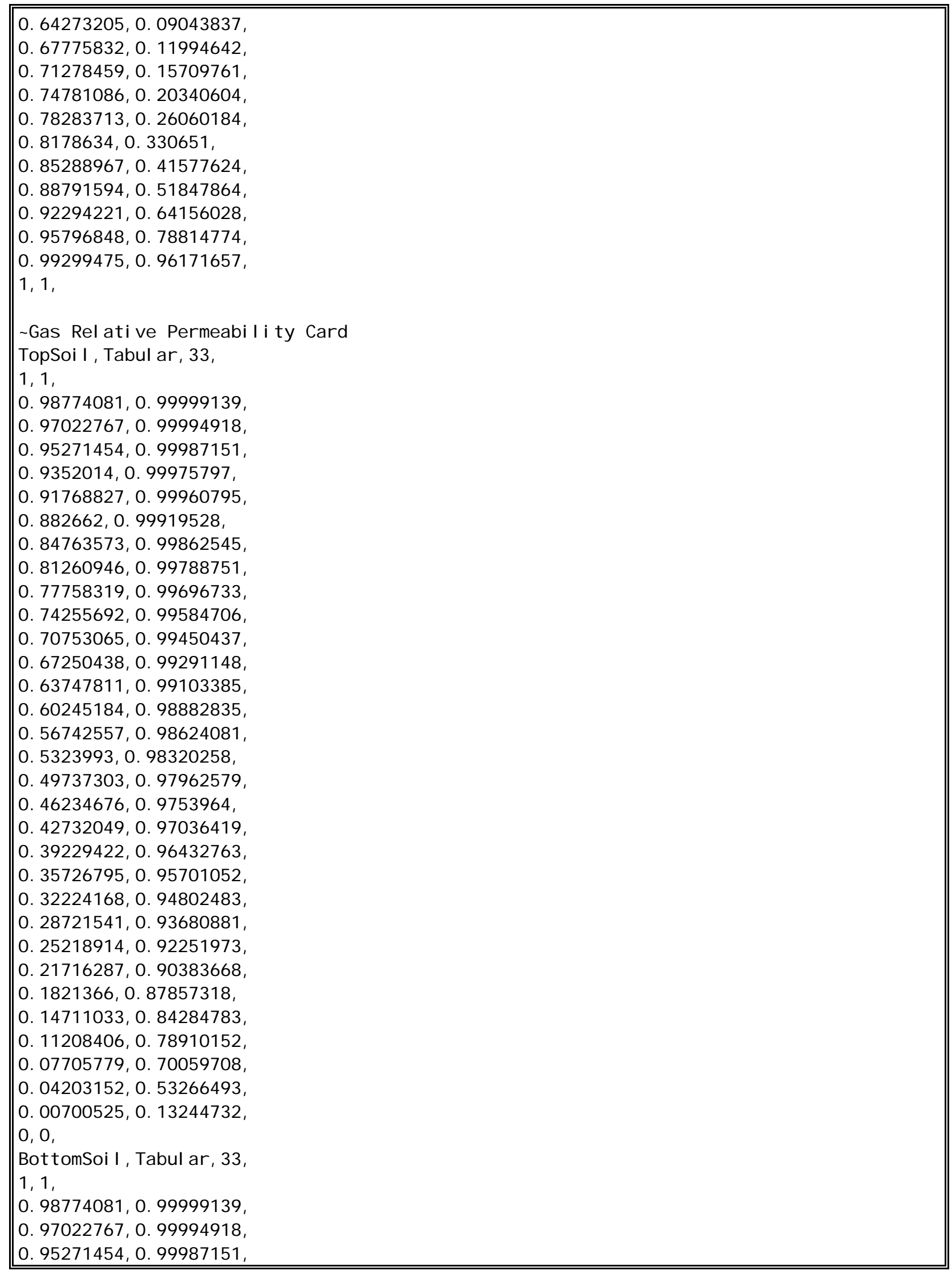




\begin{tabular}{|c|c|}
\hline 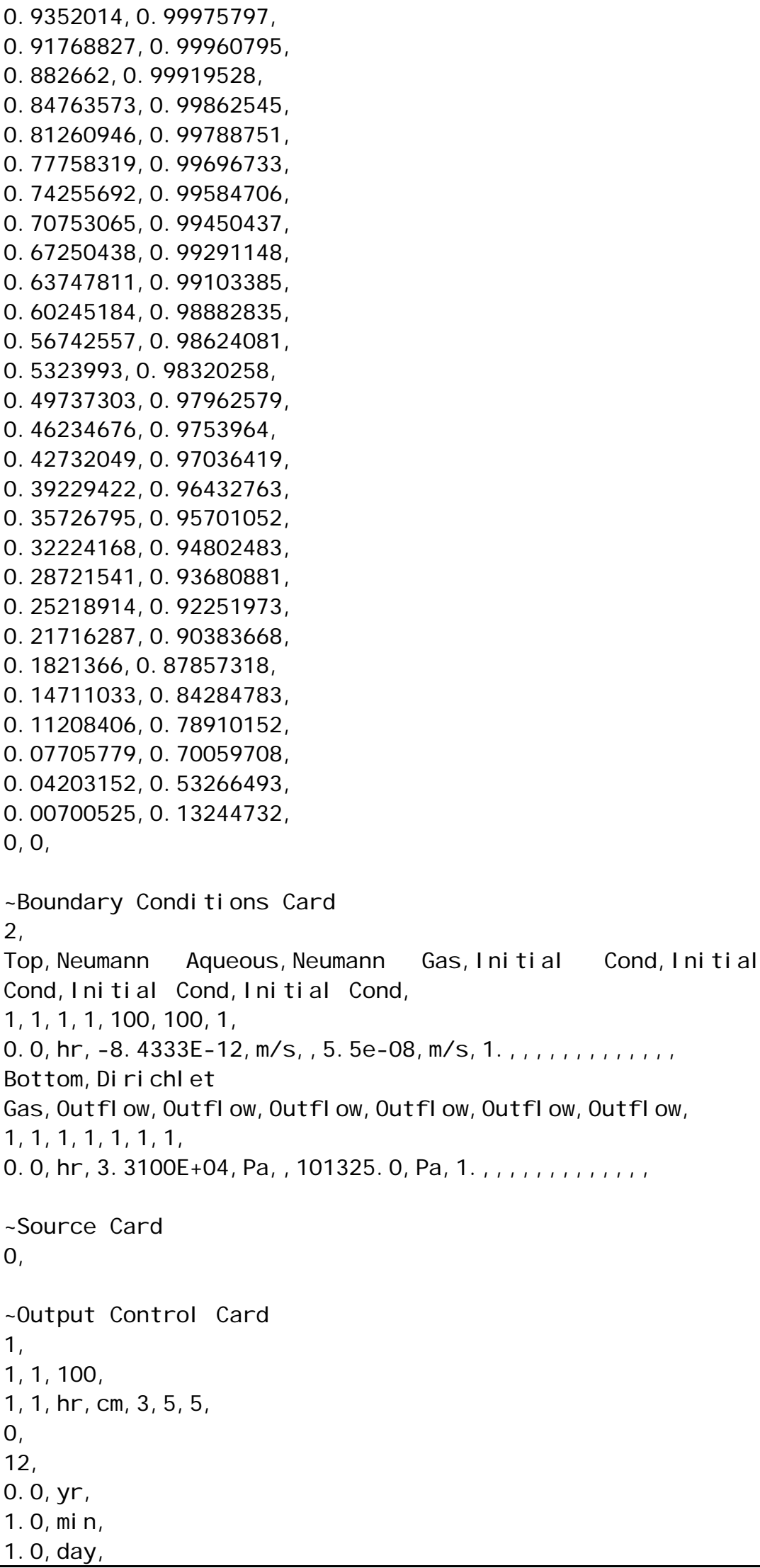 & Cond, Initial Cond, Initial \\
\hline
\end{tabular}




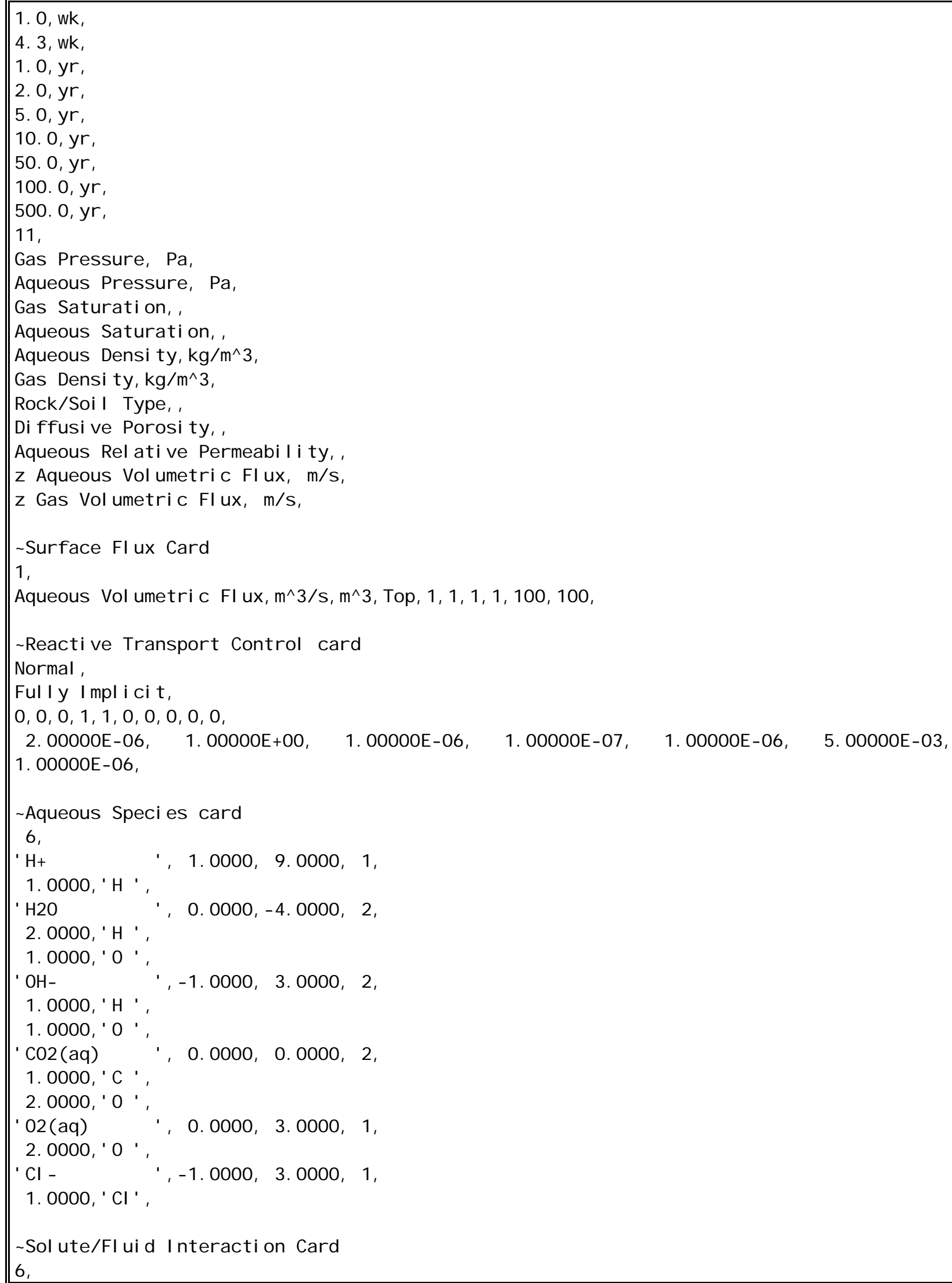




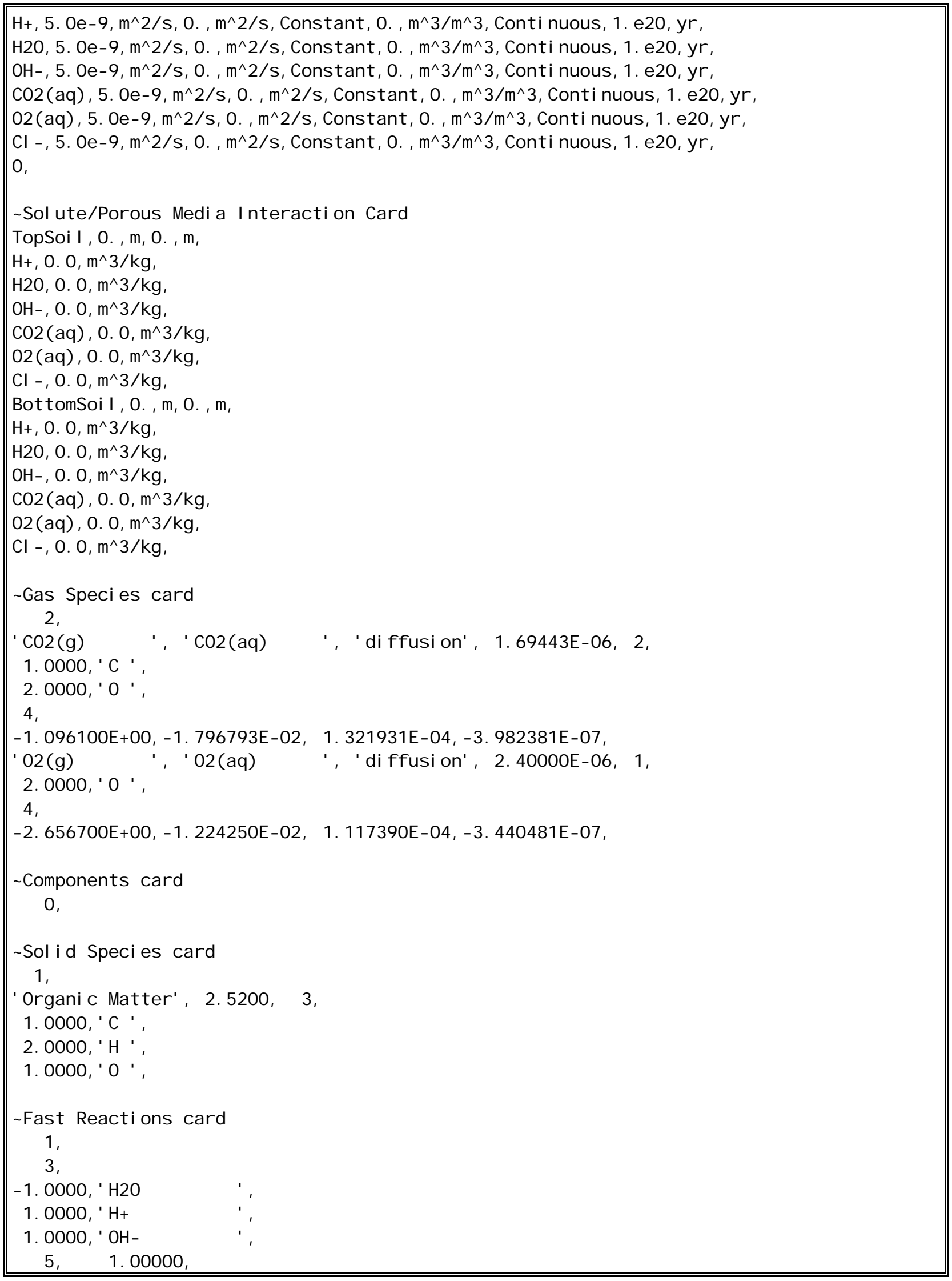




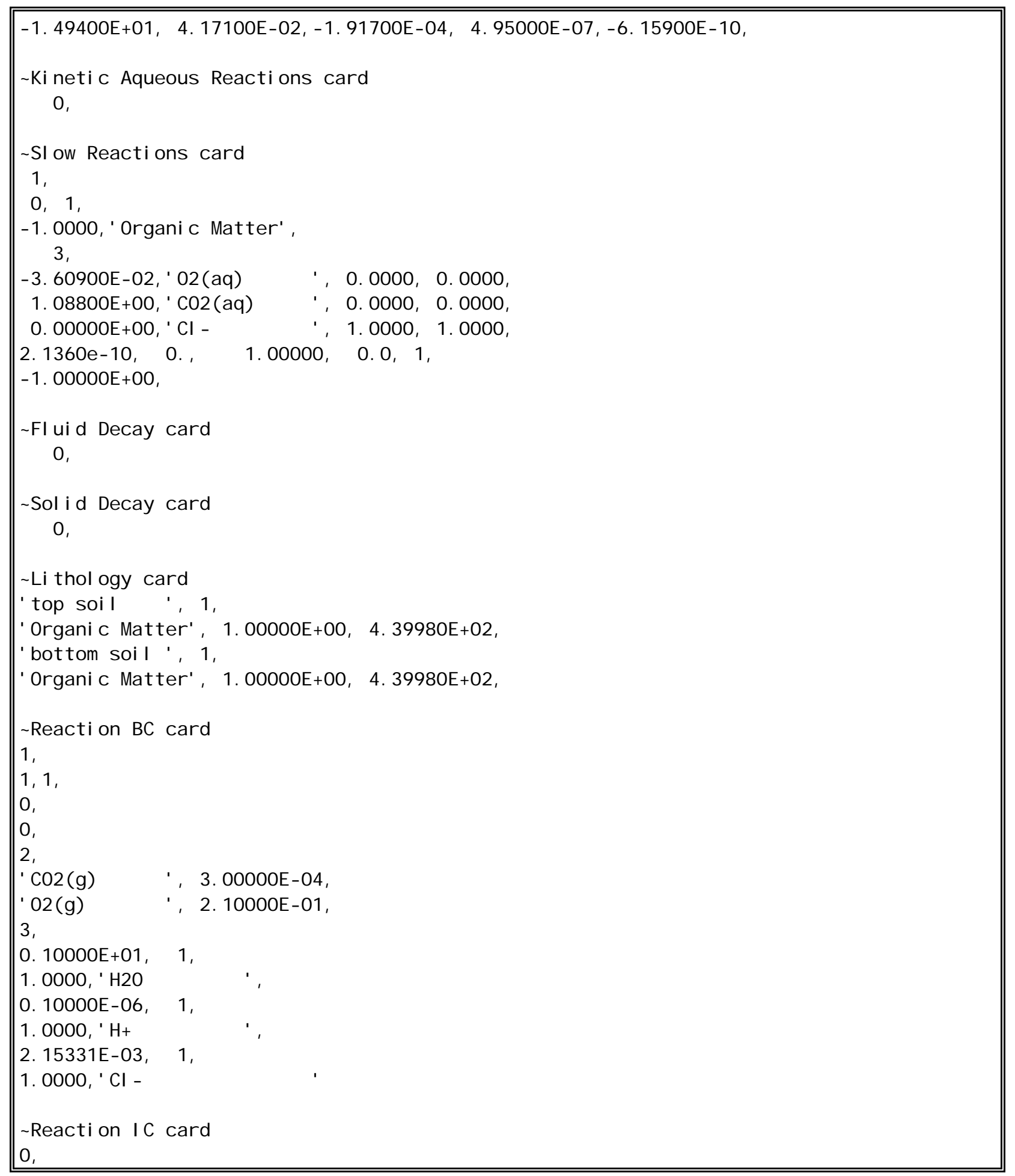




\section{C.3 STORM Input File for Quartz Dissolution Test Case}

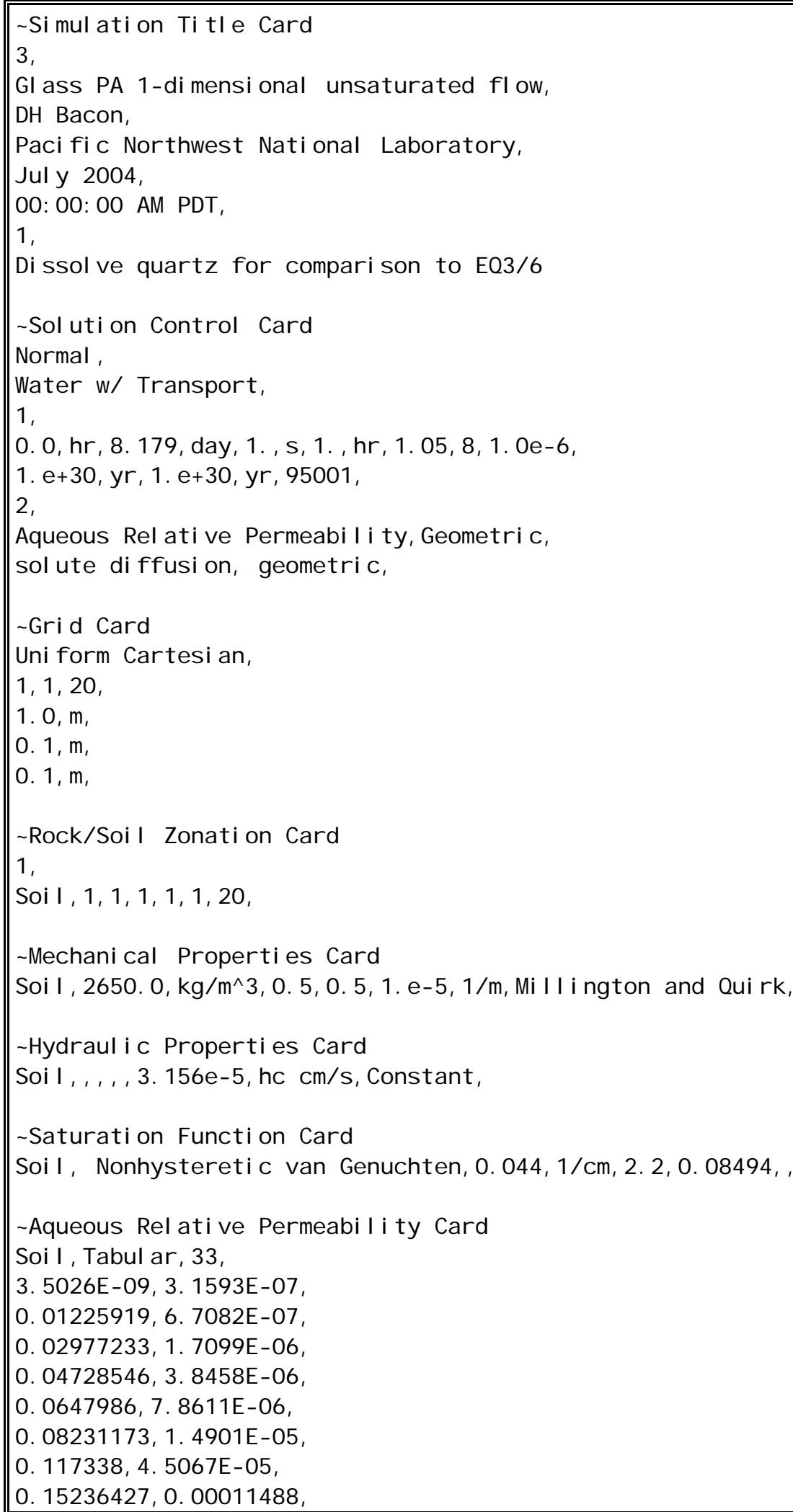




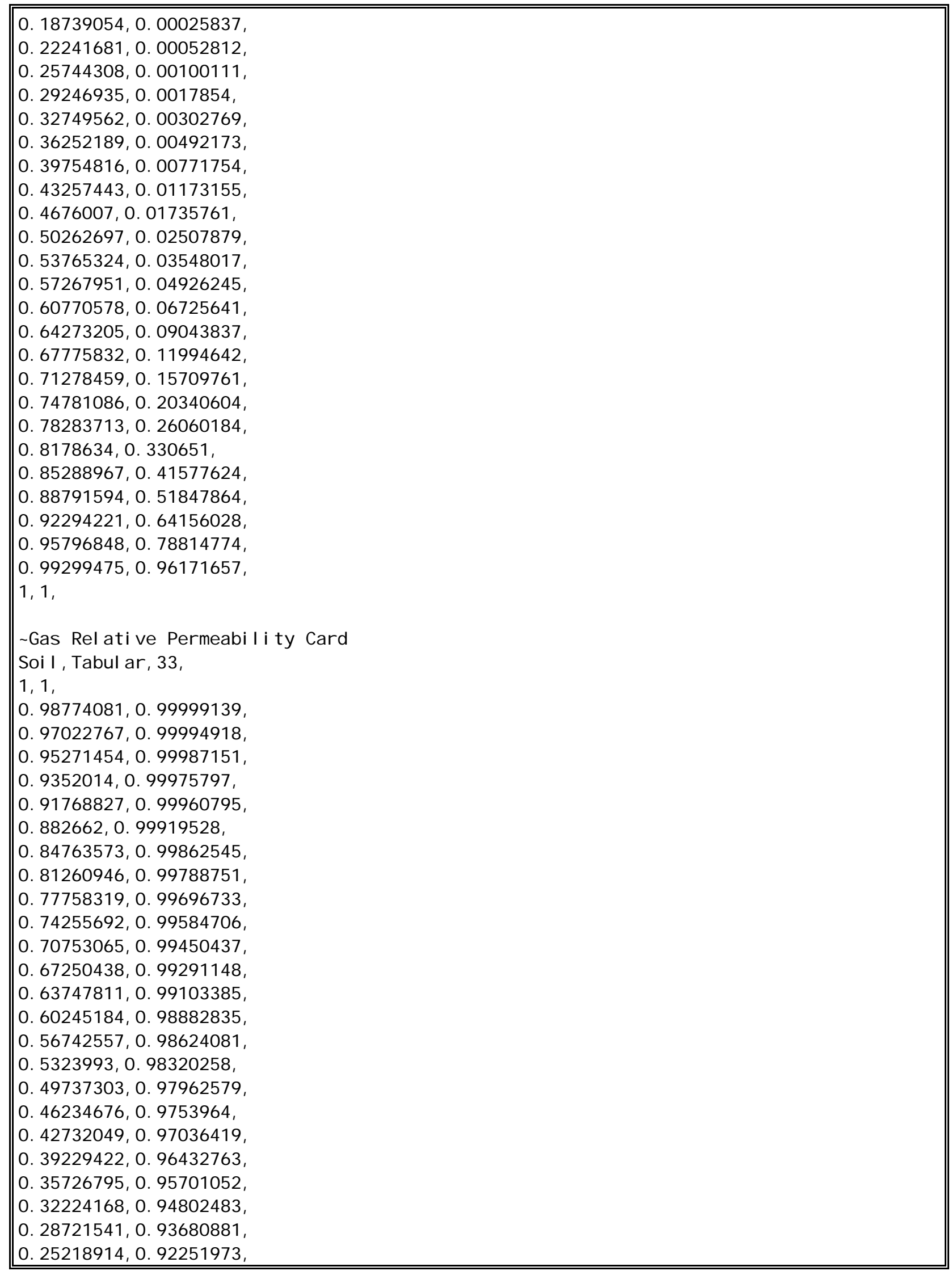

C.14 


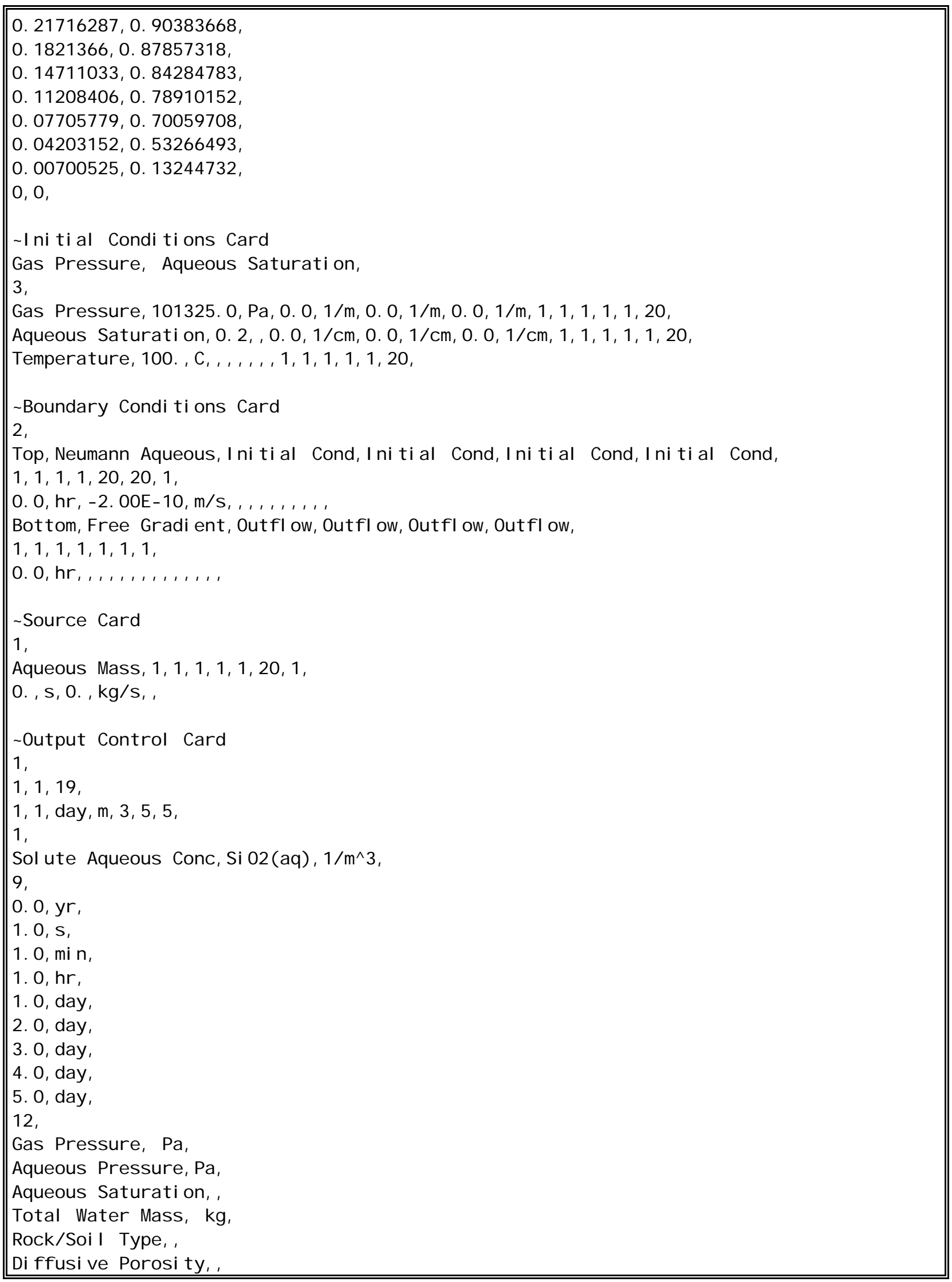




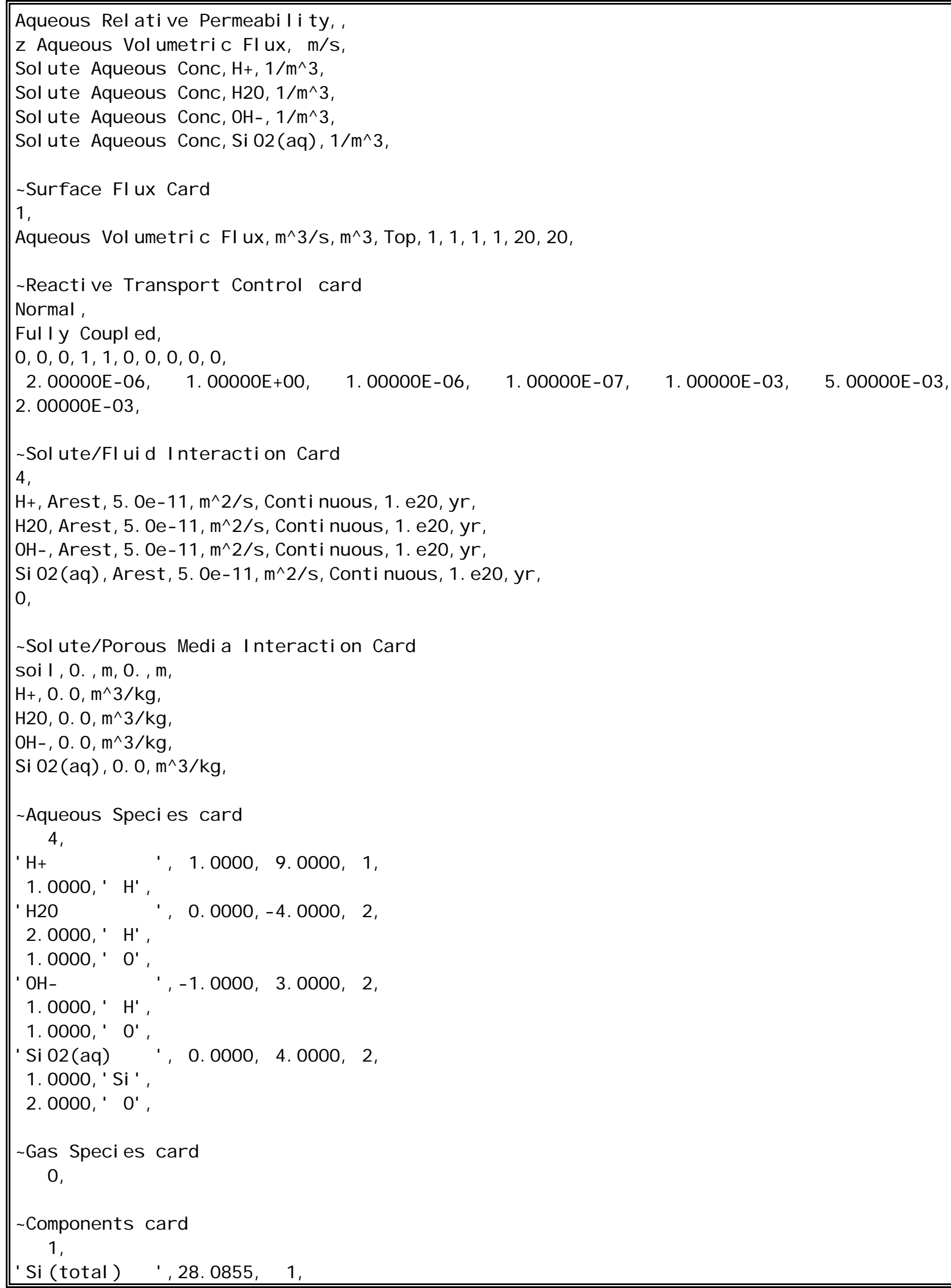




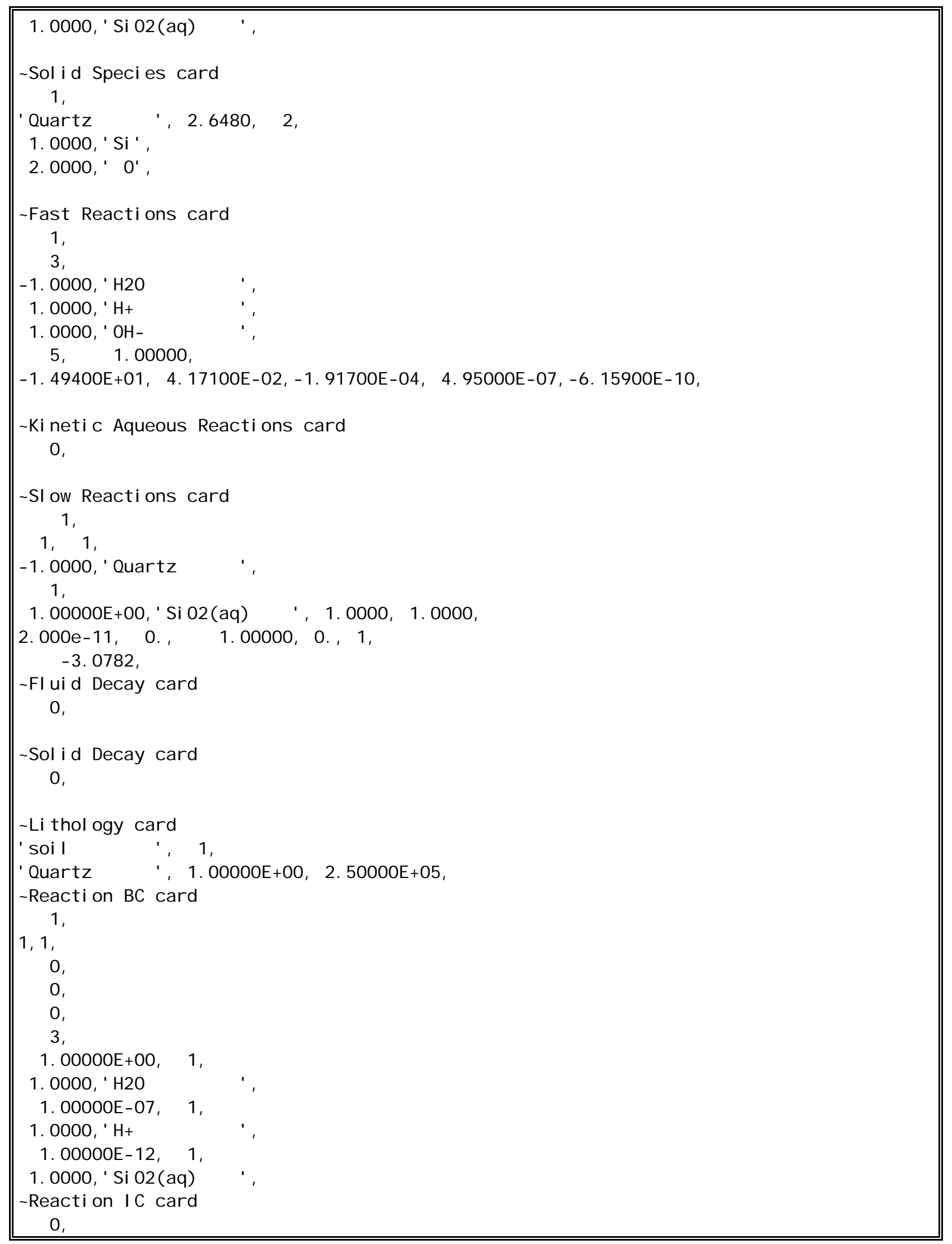




\section{C.4 STORM Input File for Calcite Precipitation Test Case}

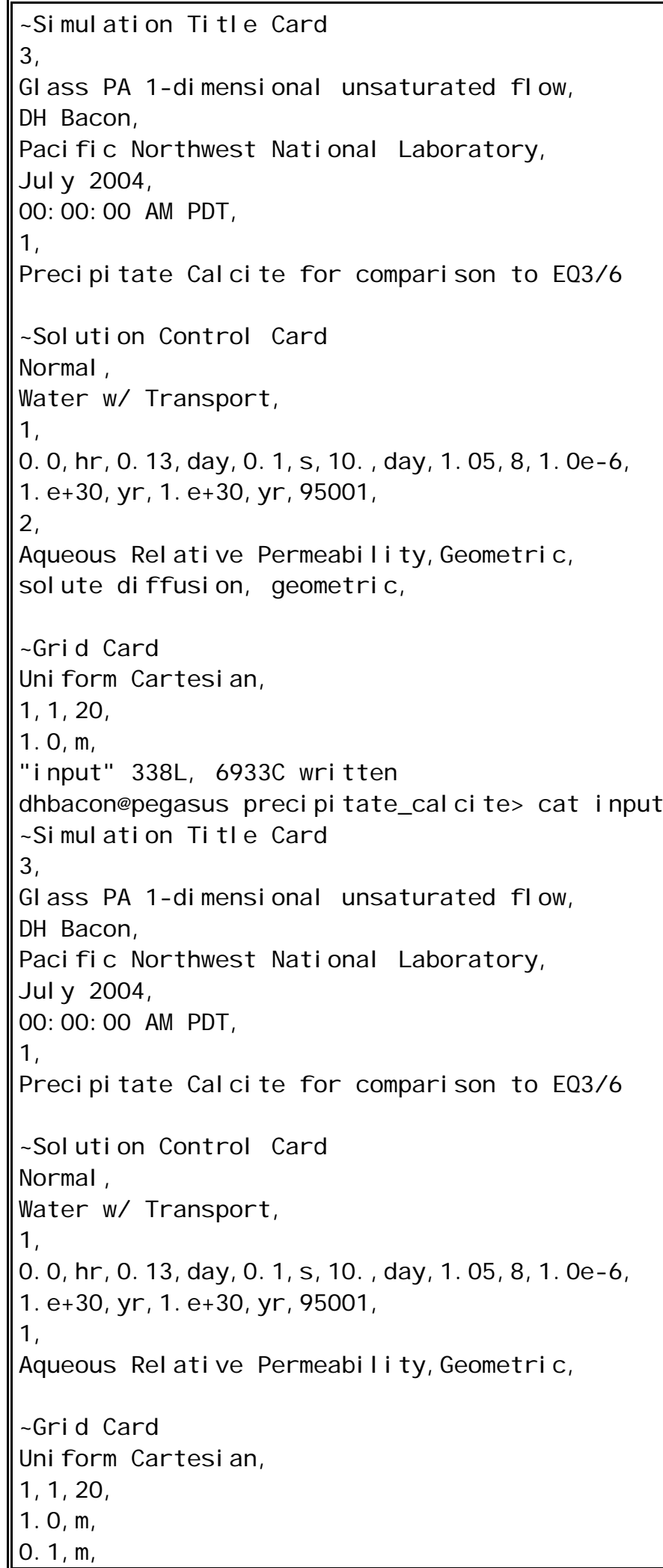




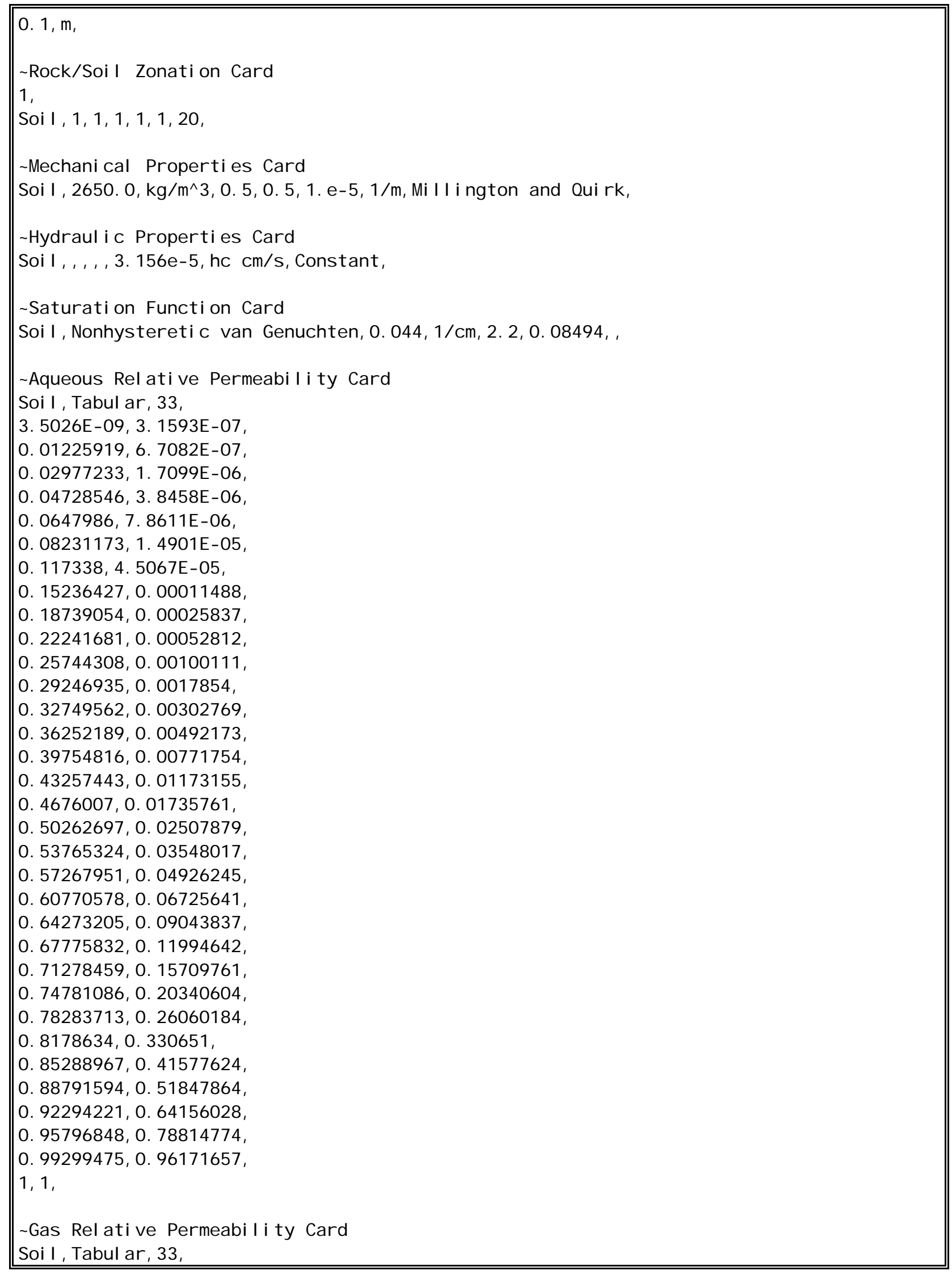




\begin{tabular}{|c|}
\hline 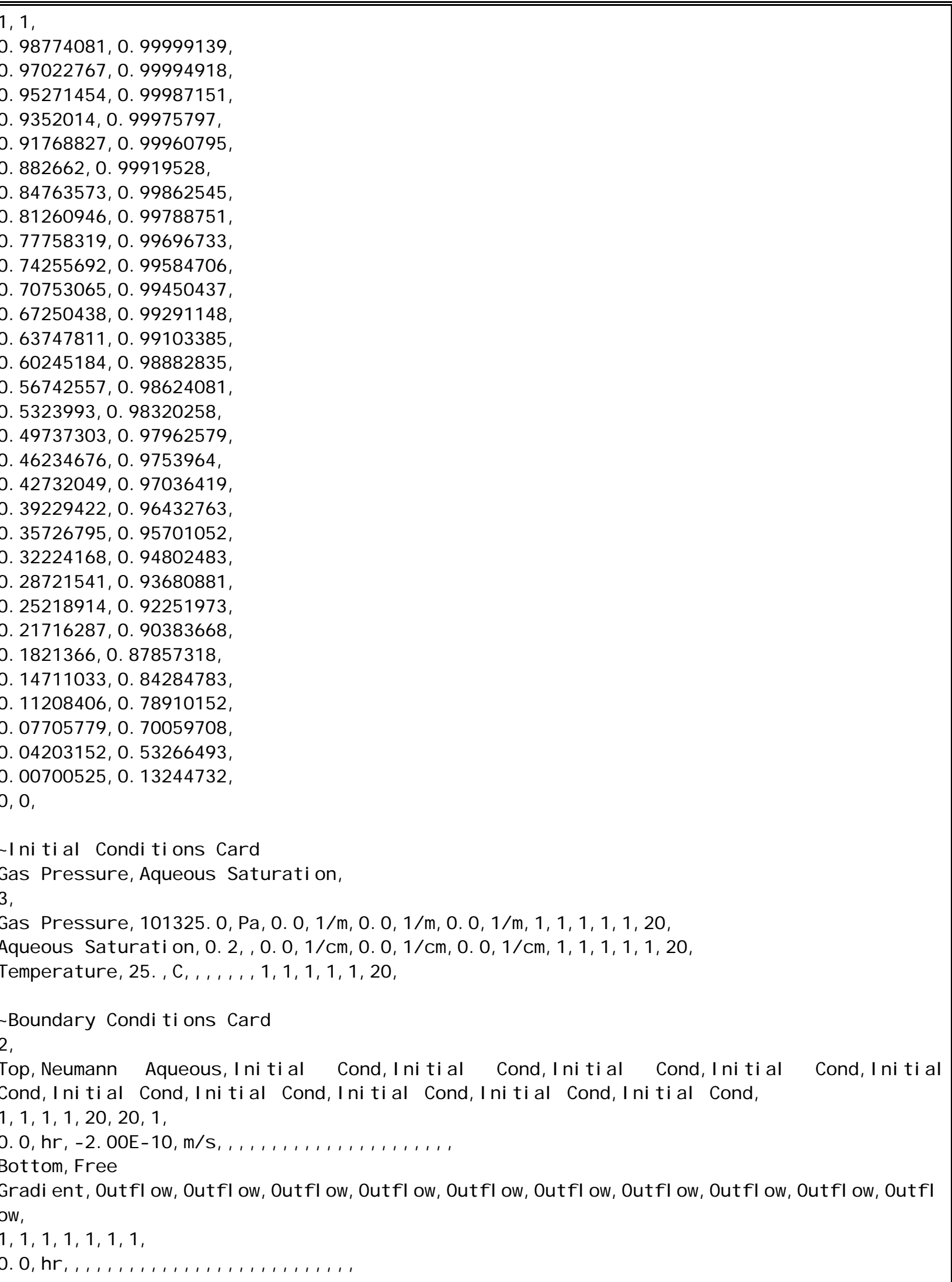 \\
\hline
\end{tabular}




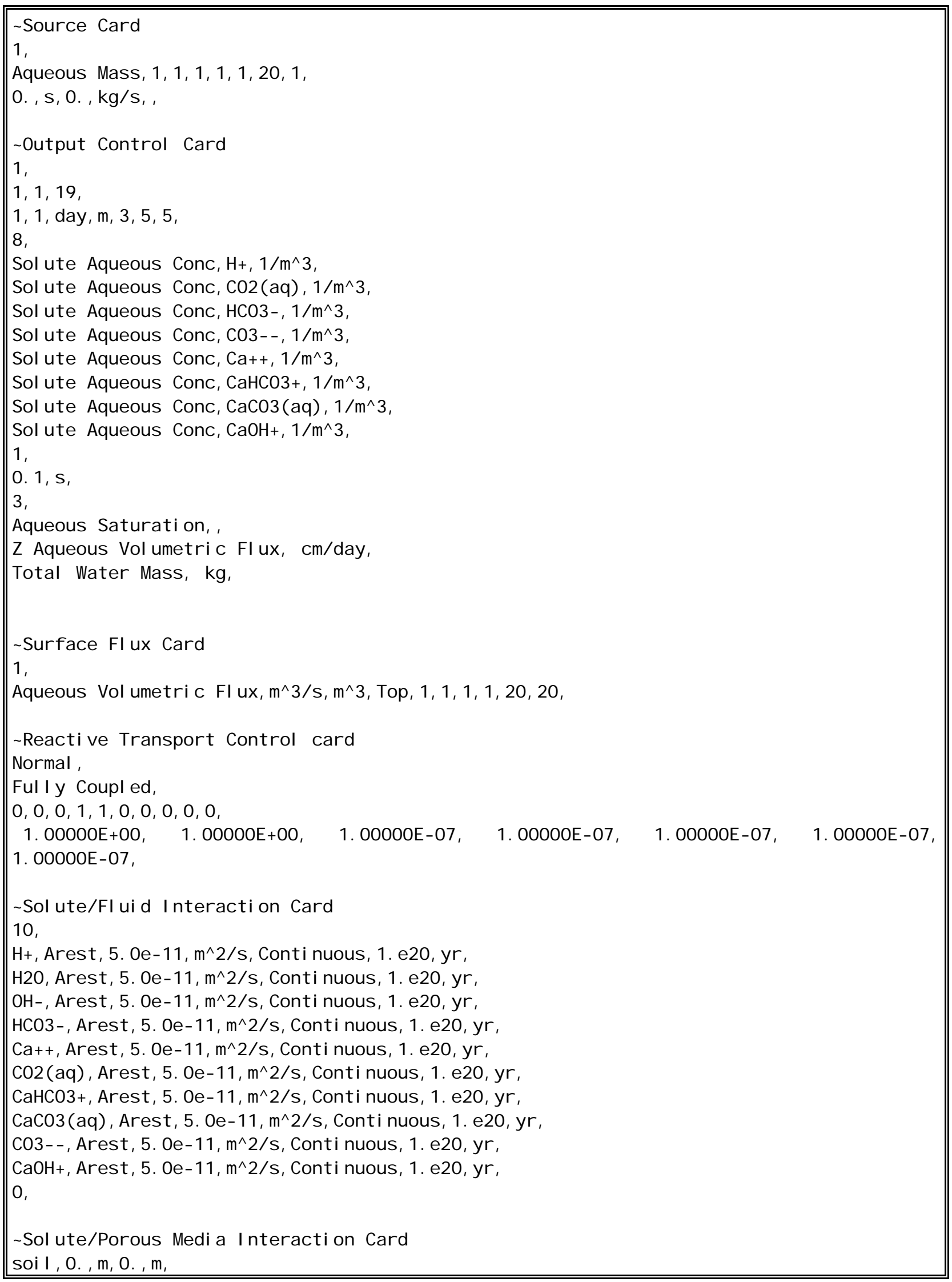




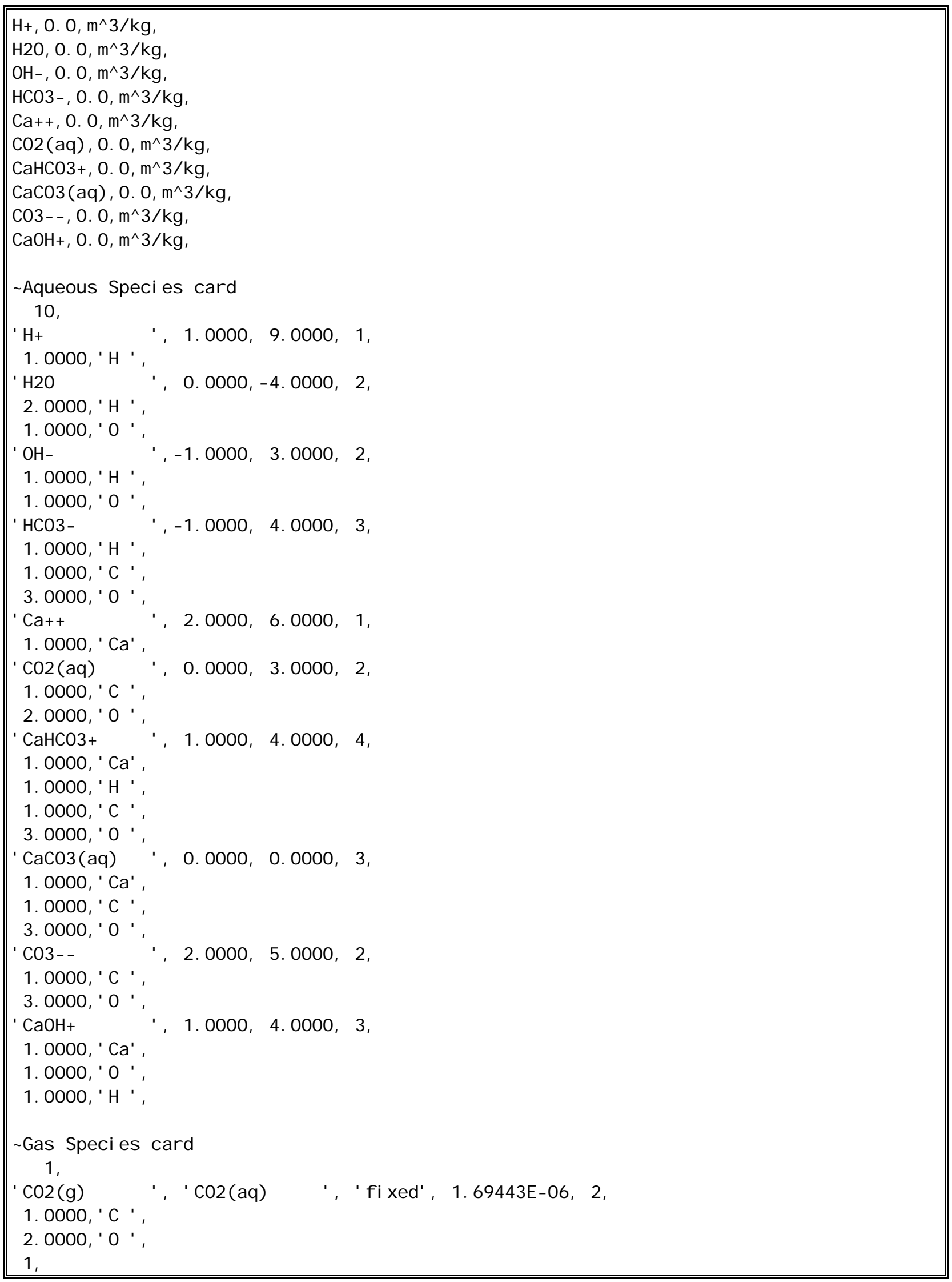




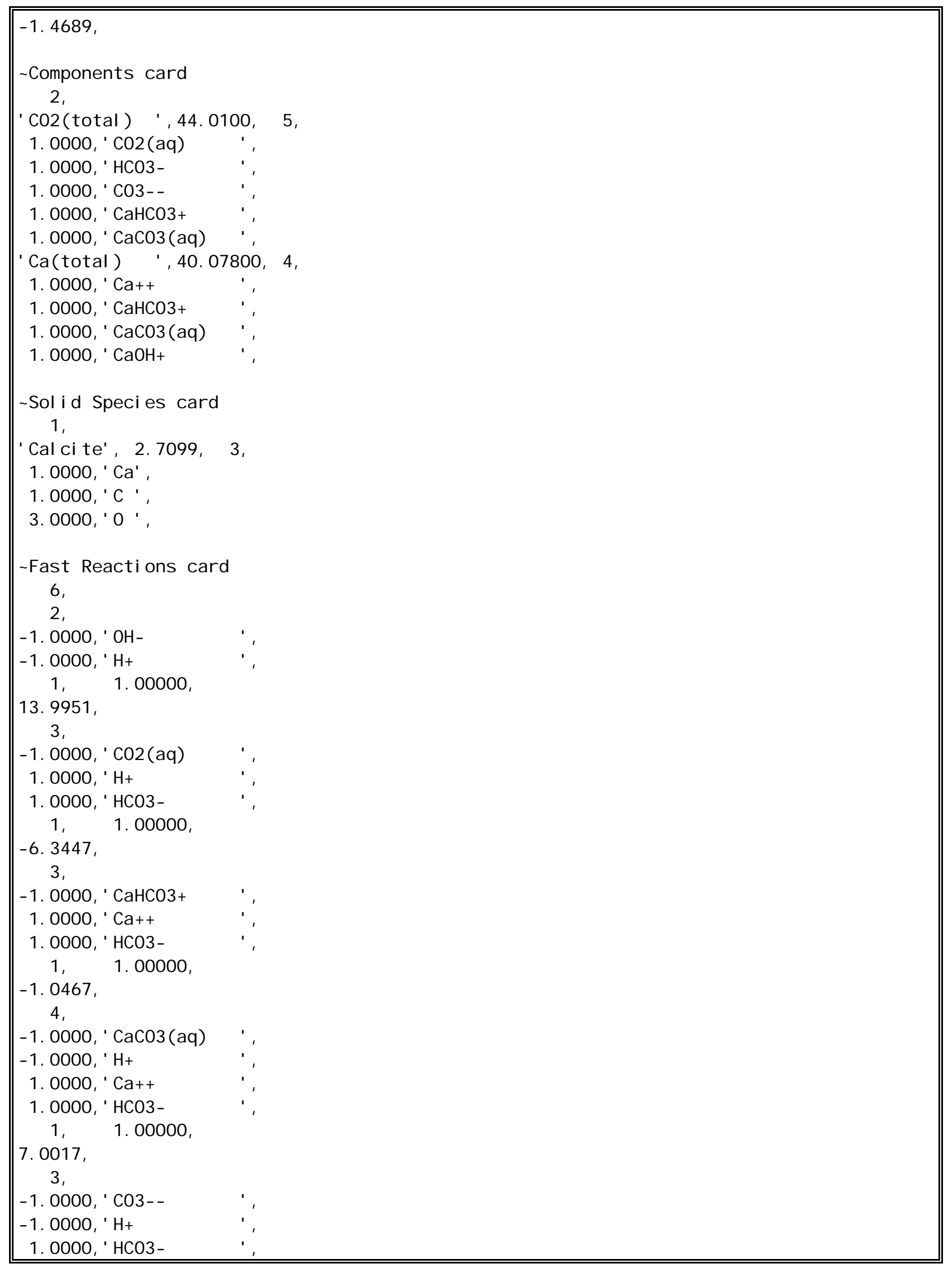




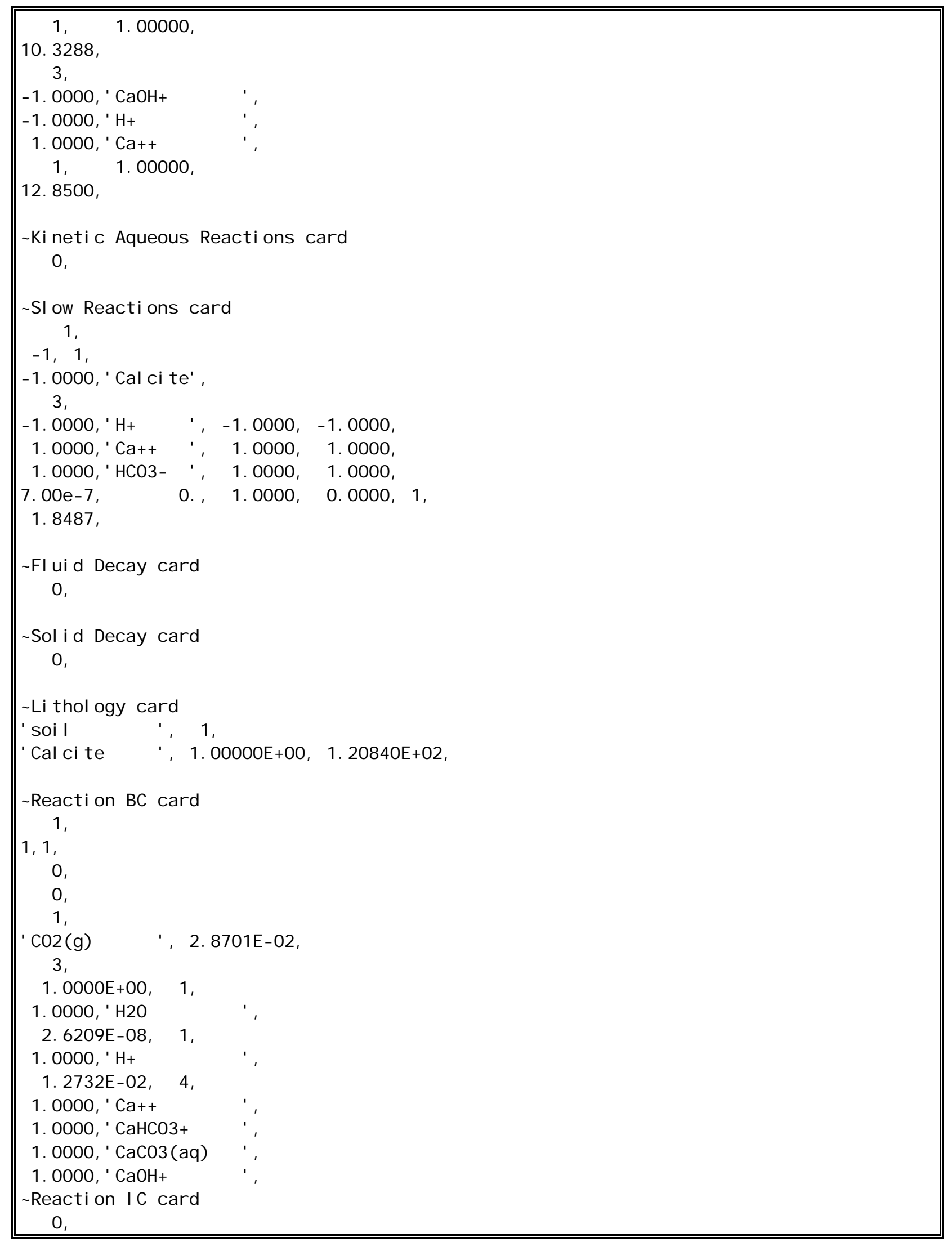




\section{C.5 STORM Input File for Example Simulation}

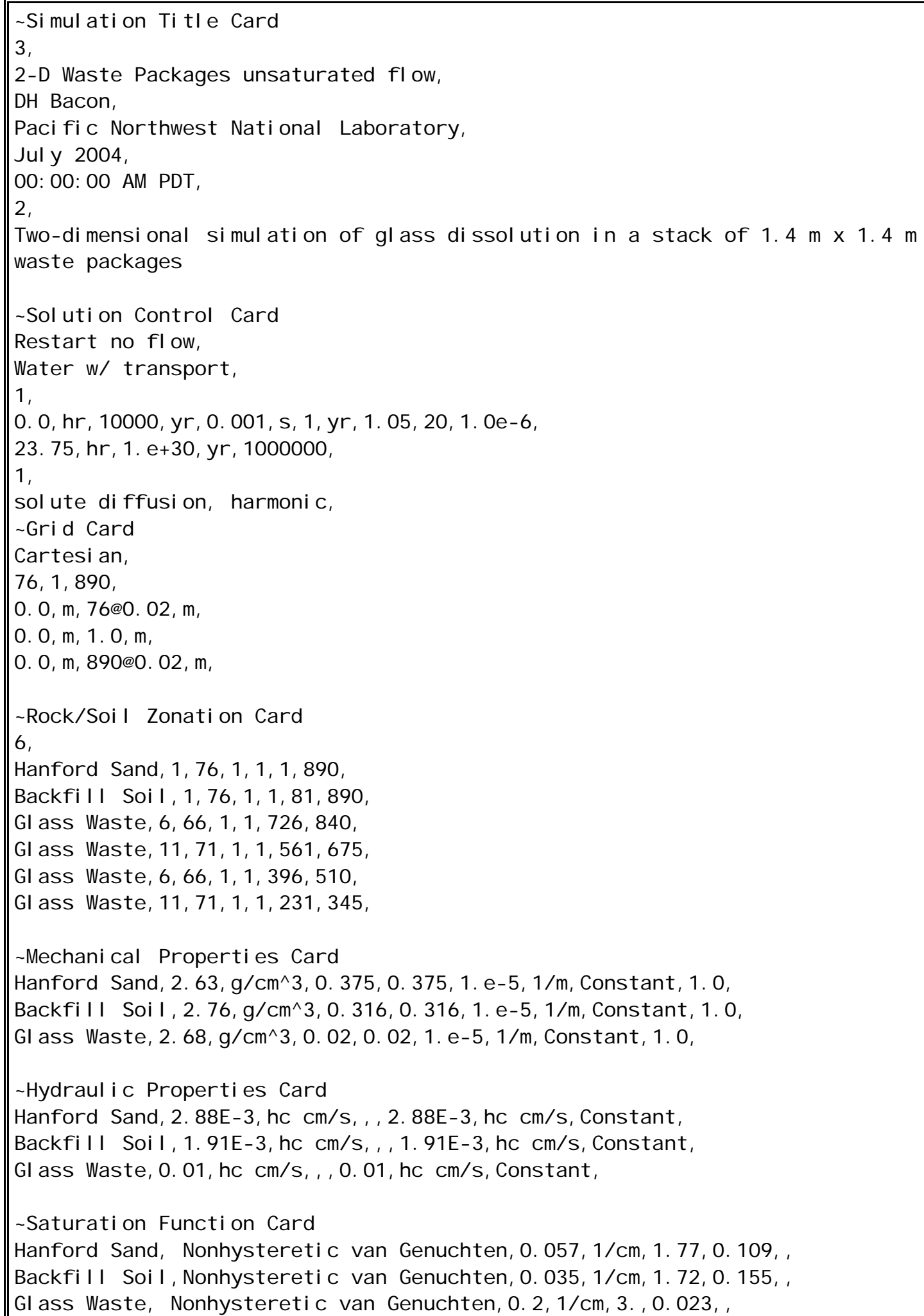




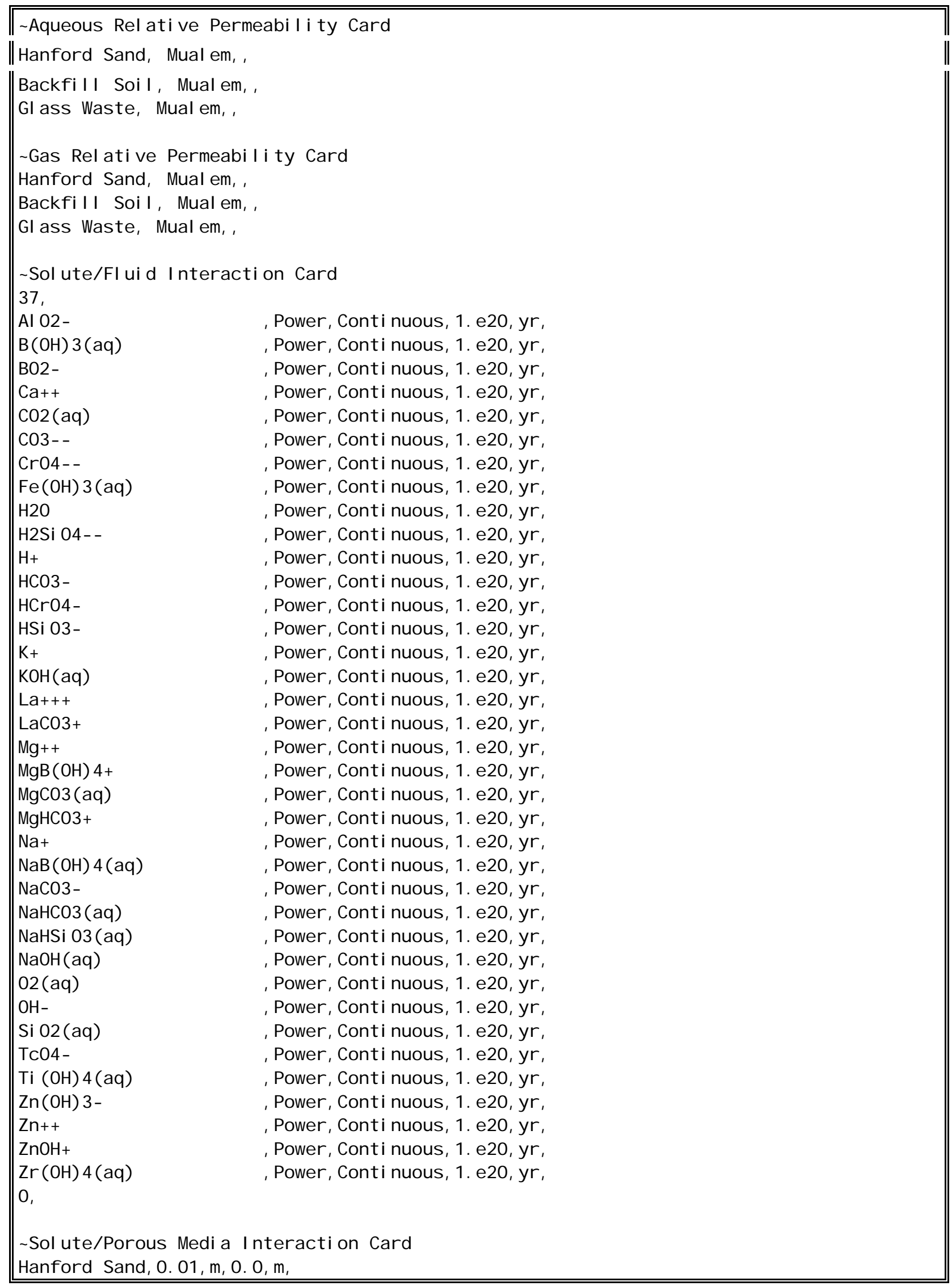




\begin{tabular}{|c|c|}
\hline Al 02 - & $, 0.0, \mathrm{~m}^{\wedge} 3 / \mathrm{kg}, 1.84 \mathrm{e}-5, \mathrm{~cm}^{\wedge} 2 / \mathrm{s}, 1.486,1.956$, \\
\hline$B(O H) 3(a q)$ & $, 0.0, \mathrm{~m}^{\wedge} 3 / \mathrm{kg}, 1.84 \mathrm{e}-5, \mathrm{~cm}^{\wedge} 2 / \mathrm{s}, 1.486,1.956$ \\
\hline $\mathrm{BO2}-$ & $, 0.0, \mathrm{~m}^{\wedge} 3 / \mathrm{kg}, 1.84 \mathrm{e}-5, \mathrm{~cm}^{\wedge} 2 / \mathrm{s}, 1.486,1.956$, \\
\hline $\mathrm{Ca}++$ & $0.0, \mathrm{~m}^{\wedge} 3 / \mathrm{kg}, 1.84 \mathrm{e}-5, \mathrm{~cm}^{\wedge} 2 / \mathrm{s}, 1.486,1.956$ \\
\hline $\cos (\mathrm{aq})$ & $, 0.0, \mathrm{~m}^{\wedge} 3 / \mathrm{kg}, 1.84 \mathrm{e}-5, \mathrm{~cm}^{\wedge} 2 / \mathrm{s}, 1.486,1.956$, \\
\hline $\mathrm{CO3-.}$ & $, 0.0, \mathrm{~m}^{\wedge} 3 / \mathrm{kg}, 1.84 \mathrm{e}-5, \mathrm{~cm}^{\wedge} 2 / \mathrm{s}, 1.486,1.956$, \\
\hline $\mathrm{CrO}-\mathrm{-}$ & $, 0.0, \mathrm{~m}^{\wedge} 3 / \mathrm{kg}, 1.84 \mathrm{e}-5, \mathrm{~cm}^{\wedge} 2 / \mathrm{s}, 1.486,1.956$, \\
\hline $\mathrm{Fe}(\mathrm{OH}) 3(\mathrm{aq})$ & $, 0.0, \mathrm{~m}^{\wedge} 3 / \mathrm{kg}, 1.84 \mathrm{e}-5, \mathrm{~cm}^{\wedge} 2 / \mathrm{s}, 1.486,1.956$, \\
\hline $\mathrm{H} 2 \mathrm{O}$ & $, 0.0, \mathrm{~m}^{\wedge} 3 / \mathrm{kg}, 1.84 \mathrm{e}-5, \mathrm{~cm}^{\wedge} 2 / \mathrm{s}, 1.486,1.956$, \\
\hline H2Si $04--$ & $, 0.0, \mathrm{~m}^{\wedge} 3 / \mathrm{kg}, 1.84 \mathrm{e}-5, \mathrm{~cm}^{\wedge} 2 / \mathrm{s}, 1.486,1.956$ \\
\hline $\mathrm{H}+$ & $, 0.0, \mathrm{~m}^{\wedge} 3 / \mathrm{kg}, 1.84 \mathrm{e}-5, \mathrm{~cm}^{\wedge} 2 / \mathrm{s}, 1.486,1.956$, \\
\hline $\mathrm{HCO}-$ & $, 0.0, \mathrm{~m}^{\wedge} 3 / \mathrm{kg}, 1.84 \mathrm{e}-5, \mathrm{~cm}^{\wedge} 2 / \mathrm{s}, 1.486,1.956$, \\
\hline $\mathrm{HCrO} O 4-$ & $, 0.0, \mathrm{~m}^{\wedge} 3 / \mathrm{kg}, 1.84 \mathrm{e}-5, \mathrm{~cm}^{\wedge} 2 / \mathrm{s}, 1.486,1.956$, \\
\hline HS I 03 - & $, 0.0, \mathrm{~m}^{\wedge} 3 / \mathrm{kg}, 1.84 \mathrm{e}-5, \mathrm{~cm}^{\wedge} 2 / \mathrm{s}, 1.486,1.956$, \\
\hline$K+$ & $, 0.0, \mathrm{~m}^{\wedge} 3 / \mathrm{kg}, 1.84 \mathrm{e}-5, \mathrm{~cm}^{\wedge} 2 / \mathrm{s}, 1.486,1.956$, \\
\hline $\mathrm{KOH}(\mathrm{aq})$ & $, 0.0, \mathrm{~m}^{\wedge} 3 / \mathrm{kg}, 1.84 \mathrm{e}-5, \mathrm{~cm}^{\wedge} 2 / \mathrm{s}, 1.486,1.956$ \\
\hline $\mathrm{La}+++$ & $, 0.0, \mathrm{~m}^{\wedge} 3 / \mathrm{kg}, 1.84 \mathrm{e}-5, \mathrm{~cm}^{\wedge} 2 / \mathrm{s}, 1.486,1.956$ \\
\hline $\mathrm{LaCO}+$ & $, 0.0, \mathrm{~m}^{\wedge} 3 / \mathrm{kg}, 1.84 \mathrm{e}-5, \mathrm{~cm}^{\wedge} 2 / \mathrm{s}, 1.486,1.956$ \\
\hline $\mathrm{Mg}++$ & $, 0.0, \mathrm{~m}^{\wedge} 3 / \mathrm{kg}, 1.84 \mathrm{e}-5, \mathrm{~cm}^{\wedge} 2 / \mathrm{s}, 1.486,1.956$, \\
\hline $\mathrm{MgB}(\mathrm{OH}) 4+$ & $, 0.0, \mathrm{~m}^{\wedge} 3 / \mathrm{kg}, 1.84 \mathrm{e}-5, \mathrm{~cm}^{\wedge} 2 / \mathrm{s}, 1.486,1.956$ \\
\hline $\operatorname{MgCO}(\mathrm{aq})$ & $, 0.0, \mathrm{~m}^{\wedge} 3 / \mathrm{kg}, 1.84 \mathrm{e}-5, \mathrm{~cm}^{\wedge} 2 / \mathrm{s}, 1.486,1.956$ \\
\hline $\mathrm{MgHCO}+$ & $, 0.0, \mathrm{~m}^{\wedge} 3 / \mathrm{kg}, 1.84 \mathrm{e}-5, \mathrm{~cm}^{\wedge} 2 / \mathrm{s}, 1.486,1.956$, \\
\hline $\mathrm{Na}+$ & $, 0.0, \mathrm{~m}^{\wedge} 3 / \mathrm{kg}, 1.84 \mathrm{e}-5, \mathrm{~cm}^{\wedge} 2 / \mathrm{s}, 1.486,1.956$, \\
\hline $\mathrm{NaB}(\mathrm{OH}) 4(\mathrm{aq})$ & $, 0.0, \mathrm{~m}^{\wedge} 3 / \mathrm{kg}, 1.84 \mathrm{e}-5, \mathrm{~cm}^{\wedge} 2 / \mathrm{s}, 1.486,1.956$ \\
\hline $\mathrm{NaCO} 3$ & $, 0.0, \mathrm{~m}^{\wedge} 3 / \mathrm{kg}, 1.84 \mathrm{e}-5, \mathrm{~cm}^{\wedge} 2 / \mathrm{s}, 1.486,1.956$ \\
\hline $\mathrm{NaHCO}$ ( a q) & $, 0.0, \mathrm{~m}^{\wedge} 3 / \mathrm{kg}, 1.84 \mathrm{e}-5, \mathrm{~cm}^{\wedge} 2 / \mathrm{s}, 1.486,1.956$, \\
\hline $\mathrm{NaHS}$ i 03 ( a q ) & $, 0.0, \mathrm{~m}^{\wedge} 3 / \mathrm{kg}, 1.84 \mathrm{e}-5, \mathrm{~cm}^{\wedge} 2 / \mathrm{s}, 1.486,1.956$ \\
\hline $\mathrm{NaOH}(\mathrm{aq})$ & $, 0.0, \mathrm{~m}^{\wedge} 3 / \mathrm{kg}, 1.84 \mathrm{e}-5, \mathrm{~cm}^{\wedge} 2 / \mathrm{s}, 1.486,1.956$, \\
\hline $02(\mathrm{aq})$ & $, 0.0, \mathrm{~m}^{\wedge} 3 / \mathrm{kg}, 1.84 \mathrm{e}-5, \mathrm{~cm}^{\wedge} 2 / \mathrm{s}, 1.486,1.956$ \\
\hline $\mathrm{OH}-$ & $, 0.0, \mathrm{~m}^{\wedge} 3 / \mathrm{kg}, 1.84 \mathrm{e}-5, \mathrm{~cm}^{\wedge} 2 / \mathrm{s}, 1.486,1.956$ \\
\hline Si $02(a q)$ & $, 0.0, \mathrm{~m}^{\wedge} 3 / \mathrm{kg}, 1.84 \mathrm{e}-5, \mathrm{~cm}^{\wedge} 2 / \mathrm{s}, 1.486,1.956$ \\
\hline TсO4- & $, 0.0, \mathrm{~m}^{\wedge} 3 / \mathrm{kg}, 1.84 \mathrm{e}-5, \mathrm{~cm}^{\wedge} 2 / \mathrm{s}, 1.486,1.956$ \\
\hline $\mathrm{Ti}(\mathrm{OH}) 4(\mathrm{aq})$ & $, 0.0, \mathrm{~m}^{\wedge} 3 / \mathrm{kg}, 1.84 \mathrm{e}-5, \mathrm{~cm}^{\wedge} 2 / \mathrm{s}, 1.486,1.956$, \\
\hline $\mathrm{Zn}(\mathrm{OH}) 3 \cdot$ & $, 0.0, \mathrm{~m}^{\wedge} 3 / \mathrm{kg}, 1.84 \mathrm{e}-5, \mathrm{~cm}^{\wedge} 2 / \mathrm{s}, 1.486,1.956$ \\
\hline$Z n++$ & $, 0.0, \mathrm{~m}^{\wedge} 3 / \mathrm{kg}, 1.84 \mathrm{e}-5, \mathrm{~cm}^{\wedge} 2 / \mathrm{s}, 1.486,1.956$ \\
\hline $\mathrm{ZnOH}+$ & $, 0.0, \mathrm{~m}^{\wedge} 3 / \mathrm{kg}, 1.84 \mathrm{e}-5, \mathrm{~cm}^{\wedge} 2 / \mathrm{s}, 1.486,1.956$ \\
\hline $\mathrm{Zr}(\mathrm{OH}) 4(\mathrm{aq})$ & $, 0.0, \mathrm{~m}^{\wedge} 3 / \mathrm{kg}, 1.84 \mathrm{e}-5, \mathrm{~cm}^{\wedge} 2 / \mathrm{s}, 1.486,1.956$ \\
\hline Backfill Soi & $0, m$ \\
\hline Al 02 & $, 0.0, \mathrm{~m}^{\wedge} 3 / \mathrm{kg}, 1.84 \mathrm{e}-5, \mathrm{~cm}^{\wedge} 2 / \mathrm{s}, 1.486,1.956$ \\
\hline$B(O H) 3(a q)$ & $, 0.0, \mathrm{~m}^{\wedge} 3 / \mathrm{kg}, 1.84 \mathrm{e}-5, \mathrm{~cm}^{\wedge} 2 / \mathrm{s}, 1.486,1.956$ \\
\hline BO2 - & $, 0.0, \mathrm{~m}^{\wedge} 3 / \mathrm{kg}, 1.84 \mathrm{e}-5, \mathrm{~cm}^{\wedge} 2 / \mathrm{s}, 1.486,1.956$ \\
\hline $\mathrm{Ca}++$ & $, 0.0, \mathrm{~m}^{\wedge} 3 / \mathrm{kg}, 1.84 \mathrm{e}-5, \mathrm{~cm}^{\wedge} 2 / \mathrm{s}, 1.486,1.956$ \\
\hline $\operatorname{co2}(\mathrm{aq})$ & $, 0.0, \mathrm{~m}^{\wedge} 3 / \mathrm{kg}, 1.84 \mathrm{e}-5, \mathrm{~cm}^{\wedge} 2 / \mathrm{s}, 1.486,1.956$ \\
\hline CO3.- & $, 0.0, \mathrm{~m}^{\wedge} 3 / \mathrm{kg}, 1.84 \mathrm{e}-5, \mathrm{~cm}^{\wedge} 2 / \mathrm{s}, 1.486,1.956$ \\
\hline $\mathrm{CrO}-\mathrm{-}$ & $, 0.0, \mathrm{~m}^{\wedge} 3 / \mathrm{kg}, 1.84 \mathrm{e}-5, \mathrm{~cm}^{\wedge} 2 / \mathrm{s}, 1.486,1.956$, \\
\hline $\mathrm{Fe}(\mathrm{OH}) 3(\mathrm{aq})$ & $, 0.0, \mathrm{~m}^{\wedge} 3 / \mathrm{kg}, 1.84 \mathrm{e}-5, \mathrm{~cm}^{\wedge} 2 / \mathrm{s}, 1.486,1.956$ \\
\hline $\mathrm{H} 2 \mathrm{O}$ & $, 0.0, \mathrm{~m}^{\wedge} 3 / \mathrm{kg}, 1.84 \mathrm{e}-5, \mathrm{~cm}^{\wedge} 2 / \mathrm{s}, 1.486,1.956$ \\
\hline H2Si $04--$ & $, 0.0, \mathrm{~m}^{\wedge} 3 / \mathrm{kg}, 1.84 \mathrm{e}-5, \mathrm{~cm}^{\wedge} 2 / \mathrm{s}, 1.486,1.956$ \\
\hline $\mathrm{H}+$ & $, 0.0, \mathrm{~m}^{\wedge} 3 / \mathrm{kg}, 1.84 \mathrm{e}-5, \mathrm{~cm}^{\wedge} 2 / \mathrm{s}, 1.486,1.956$ \\
\hline $\mathrm{HCO}-$ & $, 0.0, \mathrm{~m}^{\wedge} 3 / \mathrm{kg}, 1.84 \mathrm{e}-5, \mathrm{~cm}^{\wedge} 2 / \mathrm{s}, 1.486,1.956$ \\
\hline $\mathrm{HCrO} O 4-$ & $, 0.0, \mathrm{~m}^{\wedge} 3 / \mathrm{kg}, 1.84 \mathrm{e}-5, \mathrm{~cm}^{\wedge} 2 / \mathrm{s}, 1.486,1.956$, \\
\hline HS i 03 - & $, 0.0, \mathrm{~m}^{\wedge} 3 / \mathrm{kg}, 1.84 \mathrm{e}-5, \mathrm{~cm}^{\wedge} 2 / \mathrm{s}, 1.486,1.956$ \\
\hline$K+$ & $, 0.0, \mathrm{~m}^{\wedge} 3 / \mathrm{kg}, 1.84 \mathrm{e}-5, \mathrm{~cm}^{\wedge} 2 / \mathrm{s}, 1.486,1.956$, \\
\hline
\end{tabular}




\begin{tabular}{|c|c|}
\hline $\mathrm{KOH}(\mathrm{aq})$ & $, 0.0, \mathrm{~m}^{\wedge} 3 / \mathrm{kg}, 1.84 \mathrm{e}-5, \mathrm{~cm}^{\wedge} 2 / \mathrm{s}, 1.486,1.956$, \\
\hline $\mathrm{La}+++$ & $, 0.0, \mathrm{~m}^{\wedge} 3 / \mathrm{kg}, 1.84 \mathrm{e}-5, \mathrm{~cm}^{\wedge} 2 / \mathrm{s}, 1.486,1.956$, \\
\hline $\mathrm{LaCO}+$ & $, 0.0, \mathrm{~m}^{\wedge} 3 / \mathrm{kg}, 1.84 \mathrm{e}-5, \mathrm{~cm}^{\wedge} 2 / \mathrm{s}, 1.486,1.956$, \\
\hline$M g++$ & $, 0.0, \mathrm{~m}^{\wedge} 3 / \mathrm{kg}, 1.84 \mathrm{e}-5, \mathrm{~cm}^{\wedge} 2 / \mathrm{s}, 1.486,1.956$, \\
\hline $\mathrm{MgB}(\mathrm{OH}) 4+$ & $, 0.0, \mathrm{~m}^{\wedge} 3 / \mathrm{kg}, 1.84 \mathrm{e}-5, \mathrm{~cm}^{\wedge} 2 / \mathrm{s}, 1.486,1.956$, \\
\hline $\operatorname{MgCO}(\mathrm{aq})$ & $, 0.0, \mathrm{~m}^{\wedge} 3 / \mathrm{kg}, 1.84 \mathrm{e}-5, \mathrm{~cm}^{\wedge} 2 / \mathrm{s}, 1.486,1.956$, \\
\hline $\mathrm{MgHCO}+$ & $, 0.0, \mathrm{~m}^{\wedge} 3 / \mathrm{kg}, 1.84 \mathrm{e}-5, \mathrm{~cm}^{\wedge} 2 / \mathrm{s}, 1.486,1.956$ \\
\hline $\mathrm{Na}+$ & $, 0.0, \mathrm{~m}^{\wedge} 3 / \mathrm{kg}, 1.84 \mathrm{e}-5, \mathrm{~cm}^{\wedge} 2 / \mathrm{s}, 1.486,1.956$ \\
\hline $\mathrm{NaB}(\mathrm{OH}) 4(\mathrm{aq})$ & $, 0.0, \mathrm{~m}^{\wedge} 3 / \mathrm{kg}, 1.84 \mathrm{e}-5, \mathrm{~cm}^{\wedge} 2 / \mathrm{s}, 1.486,1.956$ \\
\hline $\mathrm{NaCO}$ & $, 0.0, \mathrm{~m}^{\wedge} 3 / \mathrm{kg}, 1.84 \mathrm{e}-5, \mathrm{~cm}^{\wedge} 2 / \mathrm{s}, 1.486,1.956$, \\
\hline $\mathrm{NaHCO}$ ( a q) & $, 0.0, \mathrm{~m}^{\wedge} 3 / \mathrm{kg}, 1.84 \mathrm{e}-5, \mathrm{~cm}^{\wedge} 2 / \mathrm{s}, 1.486,1.956$, \\
\hline NaHSi 03 ( aq) & $, 0.0, \mathrm{~m}^{\wedge} 3 / \mathrm{kg}, 1.84 \mathrm{e}-5, \mathrm{~cm}^{\wedge} 2 / \mathrm{s}, 1.486,1.956$, \\
\hline $\mathrm{NaOH}(\mathrm{aq})$ & $, 0.0, \mathrm{~m}^{\wedge} 3 / \mathrm{kg}, 1.84 \mathrm{e}-5, \mathrm{~cm}^{\wedge} 2 / \mathrm{s}, 1.486,1.956$, \\
\hline $02(\mathrm{aq})$ & $, 0.0, \mathrm{~m}^{\wedge} 3 / \mathrm{kg}, 1.84 \mathrm{e}-5, \mathrm{~cm}^{\wedge} 2 / \mathrm{s}, 1.486,1.956$, \\
\hline $\mathrm{OH}-$ & $, 0.0, \mathrm{~m}^{\wedge} 3 / \mathrm{kg}, 1.84 \mathrm{e}-5, \mathrm{~cm}^{\wedge} 2 / \mathrm{s}, 1.486,1.956$ \\
\hline Si $02(\mathrm{aq})$ & $, 0.0, \mathrm{~m}^{\wedge} 3 / \mathrm{kg}, 1.84 \mathrm{e}-5, \mathrm{~cm}^{\wedge} 2 / \mathrm{s}, 1.486,1.956$, \\
\hline TCO4- & $, 0.0, \mathrm{~m}^{\wedge} 3 / \mathrm{kg}, 1.84 \mathrm{e}-5, \mathrm{~cm}^{\wedge} 2 / \mathrm{s}, 1.486,1.956$, \\
\hline $\mathrm{Ti}(\mathrm{OH}) 4(\mathrm{aq})$ & $, 0.0, \mathrm{~m}^{\wedge} 3 / \mathrm{kg}, 1.84 \mathrm{e}-5, \mathrm{~cm}^{\wedge} 2 / \mathrm{s}, 1.486,1.956$, \\
\hline $\mathrm{Zn}(\mathrm{OH}) 3 \cdot$ & $, 0.0, \mathrm{~m}^{\wedge} 3 / \mathrm{kg}, 1.84 \mathrm{e}-5, \mathrm{~cm}^{\wedge} 2 / \mathrm{s}, 1.486,1.956$, \\
\hline$Z n++$ & $, 0.0, \mathrm{~m}^{\wedge} 3 / \mathrm{kg}, 1.84 \mathrm{e}-5, \mathrm{~cm}^{\wedge} 2 / \mathrm{s}, 1.486,1.956$, \\
\hline $\mathrm{ZnOH}+$ & $, 0.0, \mathrm{~m}^{\wedge} 3 / \mathrm{kg}, 1.84 \mathrm{e}-5, \mathrm{~cm}^{\wedge} 2 / \mathrm{s}, 1.486,1.956$, \\
\hline $\mathrm{Zr}(\mathrm{OH}) 4(\mathrm{aq})$ & $, 0.0, \mathrm{~m}^{\wedge} 3 / \mathrm{kg}, 1.84 \mathrm{e}-5, \mathrm{~cm}^{\wedge} 2 / \mathrm{s}, 1.486,1.956$ \\
\hline Gl ass Waste, & $\mathrm{m}$ \\
\hline Al 02 & $, 0.0, \mathrm{~m}^{\wedge} 3 / \mathrm{kg}, 1.84 \mathrm{e}-5, \mathrm{~cm}^{\wedge} 2 / \mathrm{s}, 1.486,1.956$ \\
\hline$B(O H) 3(a q)$ & $, 0.0, \mathrm{~m}^{\wedge} 3 / \mathrm{kg}, 1.84 \mathrm{e}-5, \mathrm{~cm}^{\wedge} 2 / \mathrm{s}, 1.486,1.956$, \\
\hline $\mathrm{BO} 2-$ & $, 0.0, \mathrm{~m}^{\wedge} 3 / \mathrm{kg}, 1.84 \mathrm{e}-5, \mathrm{~cm}^{\wedge} 2 / \mathrm{s}, 1.486,1.956$, \\
\hline $\mathrm{Ca}++$ & $, 0.0, \mathrm{~m}^{\wedge} 3 / \mathrm{kg}, 1.84 \mathrm{e}-5, \mathrm{~cm}^{\wedge} 2 / \mathrm{s}, 1.486,1.956$, \\
\hline $\cos (\mathrm{aq})$ & $, 0.0, \mathrm{~m}^{\wedge} 3 / \mathrm{kg}, 1.84 \mathrm{e}-5, \mathrm{~cm}^{\wedge} 2 / \mathrm{s}, 1.486,1.956$, \\
\hline $\mathrm{CO3}-\cdot$ & $, 0.0, \mathrm{~m}^{\wedge} 3 / \mathrm{kg}, 1.84 \mathrm{e}-5, \mathrm{~cm}^{\wedge} 2 / \mathrm{s}, 1.486,1.956$ \\
\hline $\mathrm{CrO}-\mathrm{H}$ & $, 0.0, \mathrm{~m}^{\wedge} 3 / \mathrm{kg}, 1.84 \mathrm{e}-5, \mathrm{~cm}^{\wedge} 2 / \mathrm{s}, 1.486,1.956$ \\
\hline $\mathrm{Fe}(\mathrm{OH}) 3(\mathrm{aq})$ & $, 0.0, \mathrm{~m}^{\wedge} 3 / \mathrm{kg}, 1.84 \mathrm{e}-5, \mathrm{~cm}^{\wedge} 2 / \mathrm{s}, 1.486,1.956$, \\
\hline $\mathrm{H} 2 \mathrm{O}$ & $, 0.0, \mathrm{~m}^{\wedge} 3 / \mathrm{kg}, 1.84 \mathrm{e}-5, \mathrm{~cm}^{\wedge} 2 / \mathrm{s}, 1.486,1.956$, \\
\hline $\mathrm{H} 2 \mathrm{Si} 04-$ & $, 0.0, \mathrm{~m}^{\wedge} 3 / \mathrm{kg}, 1.84 \mathrm{e}-5, \mathrm{~cm}^{\wedge} 2 / \mathrm{s}, 1.486,1.956$, \\
\hline$H+$ & $, 0.0, \mathrm{~m}^{\wedge} 3 / \mathrm{kg}, 1.84 \mathrm{e}-5, \mathrm{~cm}^{\wedge} 2 / \mathrm{s}, 1.486,1.956$, \\
\hline $\mathrm{HCO3-}$ & $, 0.0, \mathrm{~m}^{\wedge} 3 / \mathrm{kg}, 1.84 \mathrm{e}-5, \mathrm{~cm}^{\wedge} 2 / \mathrm{s}, 1.486,1.956$, \\
\hline $\mathrm{HCrO} 04-$ & $, 0.0, \mathrm{~m}^{\wedge} 3 / \mathrm{kg}, 1.84 \mathrm{e}-5, \mathrm{~cm}^{\wedge} 2 / \mathrm{s}, 1.486,1.956$, \\
\hline HS i 03 - & $, 0.0, \mathrm{~m}^{\wedge} 3 / \mathrm{kg}, 1.84 \mathrm{e}-5, \mathrm{~cm}^{\wedge} 2 / \mathrm{s}, 1.486,1.956$ \\
\hline$K+$ & $, 0.0, \mathrm{~m}^{\wedge} 3 / \mathrm{kg}, 1.84 \mathrm{e}-5, \mathrm{~cm}^{\wedge} 2 / \mathrm{s}, 1.486,1.956$, \\
\hline $\mathrm{KOH}(\mathrm{aq})$ & $, 0.0, \mathrm{~m}^{\wedge} 3 / \mathrm{kg}, 1.84 \mathrm{e}-5, \mathrm{~cm}^{\wedge} 2 / \mathrm{s}, 1.486,1.956$, \\
\hline$L a+++$ & $, 0.0, \mathrm{~m}^{\wedge} 3 / \mathrm{kg}, 1.84 \mathrm{e}-5, \mathrm{~cm}^{\wedge} 2 / \mathrm{s}, 1.486,1.956$, \\
\hline $\mathrm{LaCO}+$ & $, 0.0, \mathrm{~m}^{\wedge} 3 / \mathrm{kg}, 1.84 \mathrm{e}-5, \mathrm{~cm}^{\wedge} 2 / \mathrm{s}, 1.486,1.956$ \\
\hline$M g++$ & $, 0.0, \mathrm{~m}^{\wedge} 3 / \mathrm{kg}, 1.84 \mathrm{e}-5, \mathrm{~cm}^{\wedge} 2 / \mathrm{s}, 1.486,1.956$, \\
\hline $\mathrm{MgB}(\mathrm{OH}) 4+$ & $, 0.0, \mathrm{~m}^{\wedge} 3 / \mathrm{kg}, 1.84 \mathrm{e}-5, \mathrm{~cm}^{\wedge} 2 / \mathrm{s}, 1.486,1.956$ \\
\hline $\operatorname{MgCO3}(\mathrm{aq})$ & $, 0.0, \mathrm{~m}^{\wedge} 3 / \mathrm{kg}, 1.84 \mathrm{e}-5, \mathrm{~cm}^{\wedge} 2 / \mathrm{s}, 1.486,1.956$ \\
\hline $\mathrm{MgHCO}+$ & $, 0.0, \mathrm{~m}^{\wedge} 3 / \mathrm{kg}, 1.84 \mathrm{e}-5, \mathrm{~cm}^{\wedge} 2 / \mathrm{s}, 1.486,1.956$ \\
\hline $\mathrm{Na}+$ & $, 0.0, \mathrm{~m}^{\wedge} 3 / \mathrm{kg}, 1.84 \mathrm{e}-5, \mathrm{~cm}^{\wedge} 2 / \mathrm{s}, 1.486,1.956$, \\
\hline $\mathrm{NaB}(\mathrm{OH}) 4(\mathrm{aq})$ & $, 0.0, \mathrm{~m}^{\wedge} 3 / \mathrm{kg}, 1.84 \mathrm{e}-5, \mathrm{~cm}^{\wedge} 2 / \mathrm{s}, 1.486,1.956$, \\
\hline $\mathrm{NaCO}-$ & $, 0.0, \mathrm{~m}^{\wedge} 3 / \mathrm{kg}, 1.84 \mathrm{e}-5, \mathrm{~cm}^{\wedge} 2 / \mathrm{s}, 1.486,1.956$, \\
\hline $\mathrm{NaHCO}(\mathrm{aq})$ & $, 0.0, \mathrm{~m}^{\wedge} 3 / \mathrm{kg}, 1.84 \mathrm{e}-5, \mathrm{~cm}^{\wedge} 2 / \mathrm{s}, 1.486,1.956$, \\
\hline $\mathrm{NaHSi} 03(\mathrm{aq})$ & $, 0.0, \mathrm{~m}^{\wedge} 3 / \mathrm{kg}, 1.84 \mathrm{e}-5, \mathrm{~cm}^{\wedge} 2 / \mathrm{s}, 1.486,1.956$ \\
\hline $\mathrm{NaOH}(\mathrm{aq})$ & $, 0.0, \mathrm{~m}^{\wedge} 3 / \mathrm{kg}, 1.84 \mathrm{e}-5, \mathrm{~cm}^{\wedge} 2 / \mathrm{s}, 1.486,1.956$ \\
\hline $02(\mathrm{aq})$ & $, 0.0, \mathrm{~m}^{\wedge} 3 / \mathrm{kg}, 1.84 \mathrm{e}-5, \mathrm{~cm}^{\wedge} 2 / \mathrm{s}, 1.486,1.956$, \\
\hline $\mathrm{OH}-$ & $, 0.0, \mathrm{~m}^{\wedge} 3 / \mathrm{kg}, 1.84 \mathrm{e}-5, \mathrm{~cm}^{\wedge} 2 / \mathrm{s}, 1.486,1.956$, \\
\hline
\end{tabular}




\begin{tabular}{|c|c|c|c|c|c|}
\hline $\begin{array}{l}\mathrm{SiO} \text { O2(aq) } \\
\mathrm{TcO}- \\
\mathrm{Ti}(\mathrm{OH}) 4(\mathrm{aq}) \\
\mathrm{Zn}(\mathrm{OH}) 3- \\
\mathrm{Zn++} \\
\mathrm{ZnOH}+ \\
\mathrm{Zr}(\mathrm{OH}) 4(\mathrm{aq})\end{array}$ & $\begin{array}{l}, 0.0, \mathrm{~m} \\
, 0.0, \mathrm{~m} \\
, 0.0, \mathrm{~m} \\
0.0, \mathrm{~m} \\
, 0.0, \mathrm{~m} \\
0.0, \mathrm{~m} \\
0.0, \mathrm{~m}\end{array}$ & $\begin{array}{l}\wedge / \mathrm{kg}, 1.84 \mathrm{e}-5, \\
\wedge \\
3 / \mathrm{kg}, 1.84 \mathrm{e}-5, \\
\wedge \\
3 / \mathrm{kg}, 1.84 \mathrm{e}-5, \\
\wedge 3 / \mathrm{kg}, 1.84 \mathrm{e}-5, \\
\wedge 3 / \mathrm{kg}, 1.84 \mathrm{e}-5, \\
\wedge 3 / \mathrm{kg}, 1.84 \mathrm{e}-5, \\
\wedge 3 / \mathrm{kg}, 1.84 \mathrm{e}-5,\end{array}$ & $\begin{array}{l}\mathrm{m}^{\wedge} 2 / \mathrm{s}, 1.486,1 . \\
\mathrm{m}^{\wedge} 2 / \mathrm{s}, 1.486,1 . \\
\mathrm{m}^{\wedge} 2 / \mathrm{s}, 1.486,1 . \\
\mathrm{m}^{\wedge} 2 / \mathrm{s}, 1.486,1 . \\
\mathrm{m}^{\wedge} 2 / \mathrm{s}, 1.486,1 . \\
\mathrm{m}^{\wedge} 2 / \mathrm{s}, 1.486,1 . \\
\mathrm{m}^{\wedge} 2 / \mathrm{s}, 1.486,1 .\end{array}$ & $\begin{array}{l}56 \\
56, \\
56, \\
56 \\
56 \\
56 \\
56\end{array}$ & \\
\hline \multicolumn{6}{|c|}{$\begin{array}{l}\text { Boundary Conditions Card } \\
2 \text {, }\end{array}$} \\
\hline Top, Neumann & Aqueous, Initial & Cond, Initial & Cond, Initial & Cond, Initial & Cond, Initial \\
\hline Cond, Initial & Cond, Initial & Cond, Initial & Cond, Initial & Cond, Initial & Cond, Initial \\
\hline Cond, Initial & Cond, Initial & Cond, Initial & Cond, Initial & Cond, Initial & Cond, Initial \\
\hline Cond, Initial & Cond, Initial & Cond, Initial & Cond, Initial & Cond, Initial & Cond, Initial \\
\hline Cond, Initial & Cond, Initial & Cond, Initial & Cond, Initial & Cond, Initial & Cond, Initial \\
\hline Cond, Initial & Cond, Initial & Cond, Initial & Cond, Initial & Cond, Initial & Cond, Initial \\
\hline Cond, Initial & Cond, Initial & Cond, Initial & Cond, Initial & Cond, Initial & Cond, Initial \\
\hline Cond, Initial & Cond, Initial & Cond, Initial & Cond, Initial & Cond, Initial & Cond, Initial \\
\hline Cond, Initial & Cond, Initial & Cond, Initial & Cond, Initial & Cond, Initial & Cond, Initial \\
\hline Cond, Initial & Cond, Initial & Cond, Initial & Cond, Initial & Cond, Initial & Cond, Initial \\
\hline Cond, Initial & Cond, Initial & Cond, Initial & Cond, Initial & Cond, Initial & Cond, Initial \\
\hline $\begin{array}{l}\text { Cond, I niti a l } \\
\text { Cond, I ni t i a I } \\
1,76,1,1,890, \\
0, y r,- \\
4,2, \mathrm{~mm} / \mathrm{yr}, .,,\end{array}$ & $\begin{array}{l}\text { Cond, Initial } \\
\text { Cond, Initial Con } \\
890,1, \\
\end{array}$ & $\begin{array}{l}\text { Cond, Initial } \\
d, \\
,, \ldots, \ldots, \ldots, \ldots\end{array}$ & Cond, Initial & Cond, Initial & Cond, Initial \\
\hline \multicolumn{6}{|c|}{$\begin{array}{l}\text {, , , , , , , , , , , , , , , , , , , , , , , , , , , , , , , , , , , , , , , , , , , , , , , , , } \\
\text { Bottom, Unit }\end{array}$} \\
\hline \multirow{3}{*}{\multicolumn{6}{|c|}{ 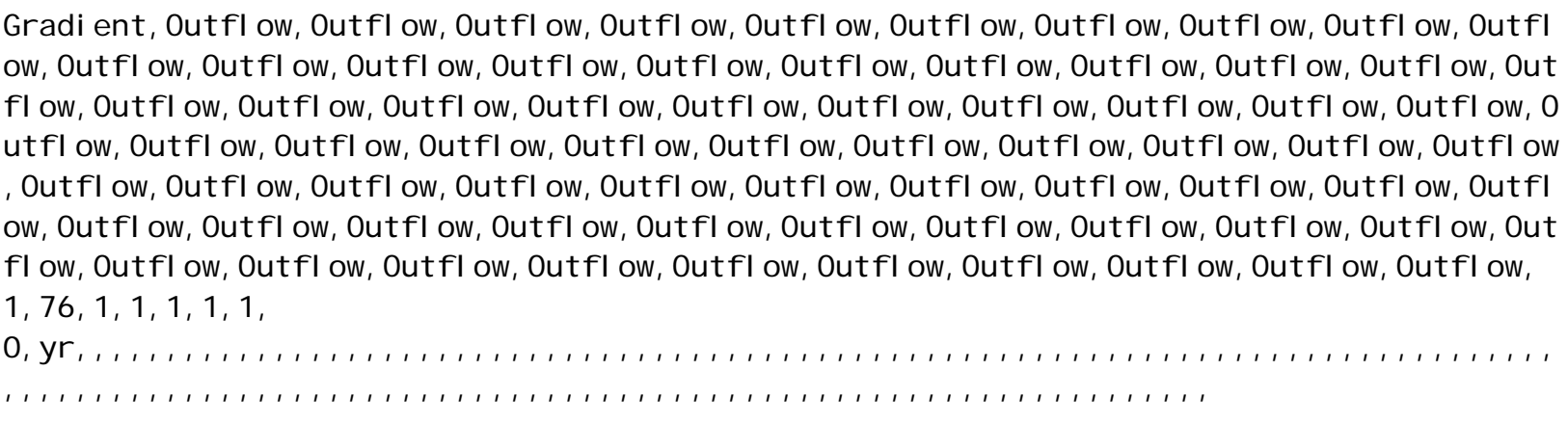 }} \\
\hline & & & & & \\
\hline & & & & & \\
\hline $\begin{array}{l}\text { Source Card } \\
1, \\
\text { Aqueous Mass, } \\
0 ., \mathrm{s}, 0 ., \mathrm{kg} / \mathrm{s},\end{array}$ & $1,76,1,1,1,890,1$ & & & & \\
\hline $\begin{array}{l}\text { output Contr } \\
0, \\
1,1, \text { yr, m, 6, } 6, \\
4, \\
\text { Aqueous Moist } \\
\text { znc Aqueous } \\
\text { Aqueous Densi } \\
\text { Sol ute Aqueou } \\
21,\end{array}$ & $\begin{array}{l}\text { ol Card } \\
6, \\
\text { ure content, } \\
\text { ol umetric FI ux, m } \\
\text { ty, kg/m^3, } \\
\text { s Conc, Tc04-, 1/ m }\end{array}$ & 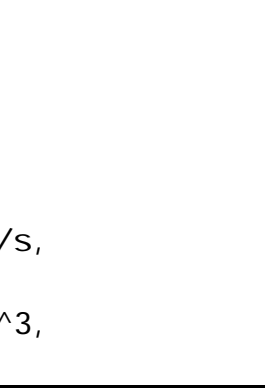 & & & \\
\hline
\end{tabular}




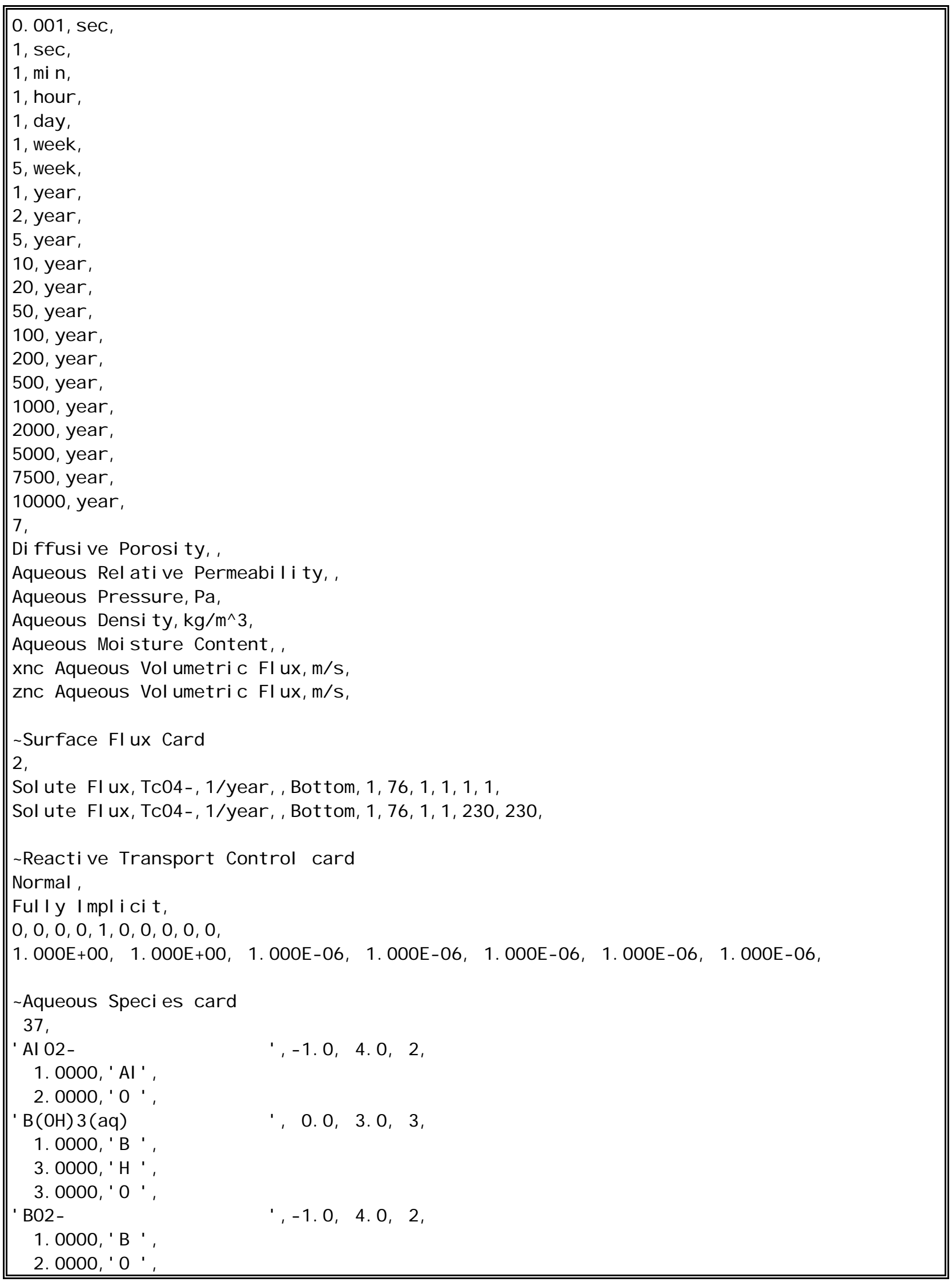




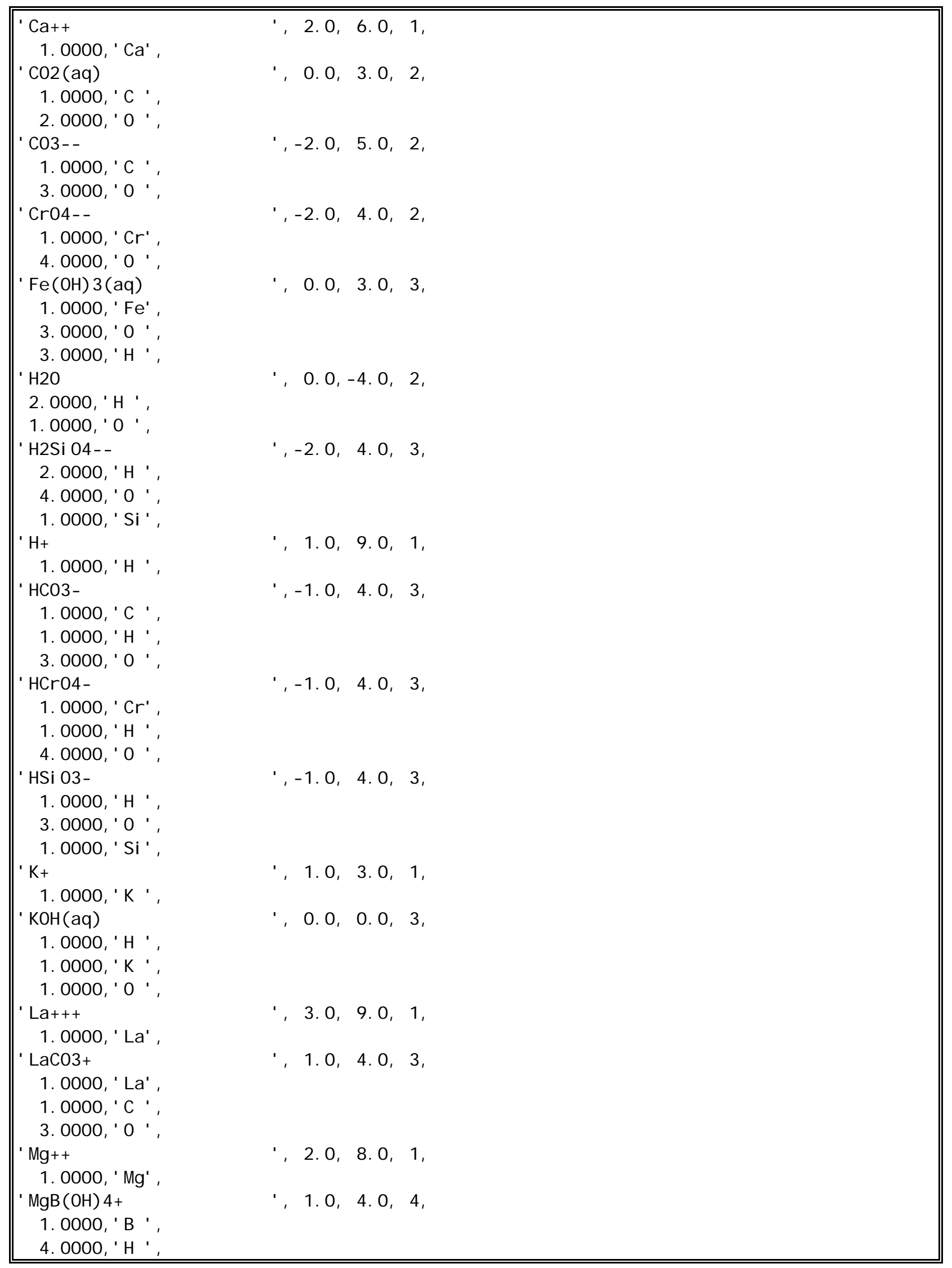




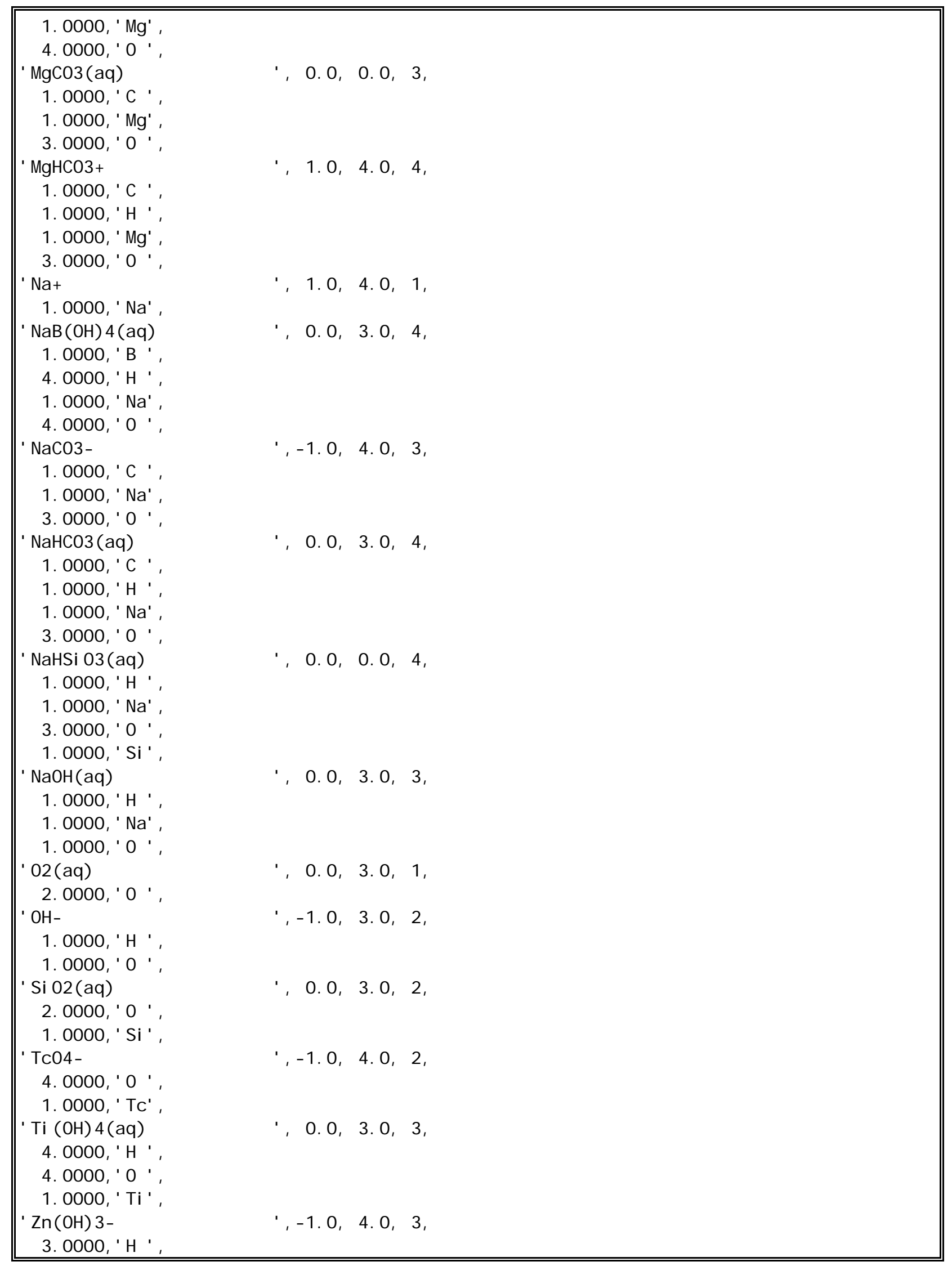




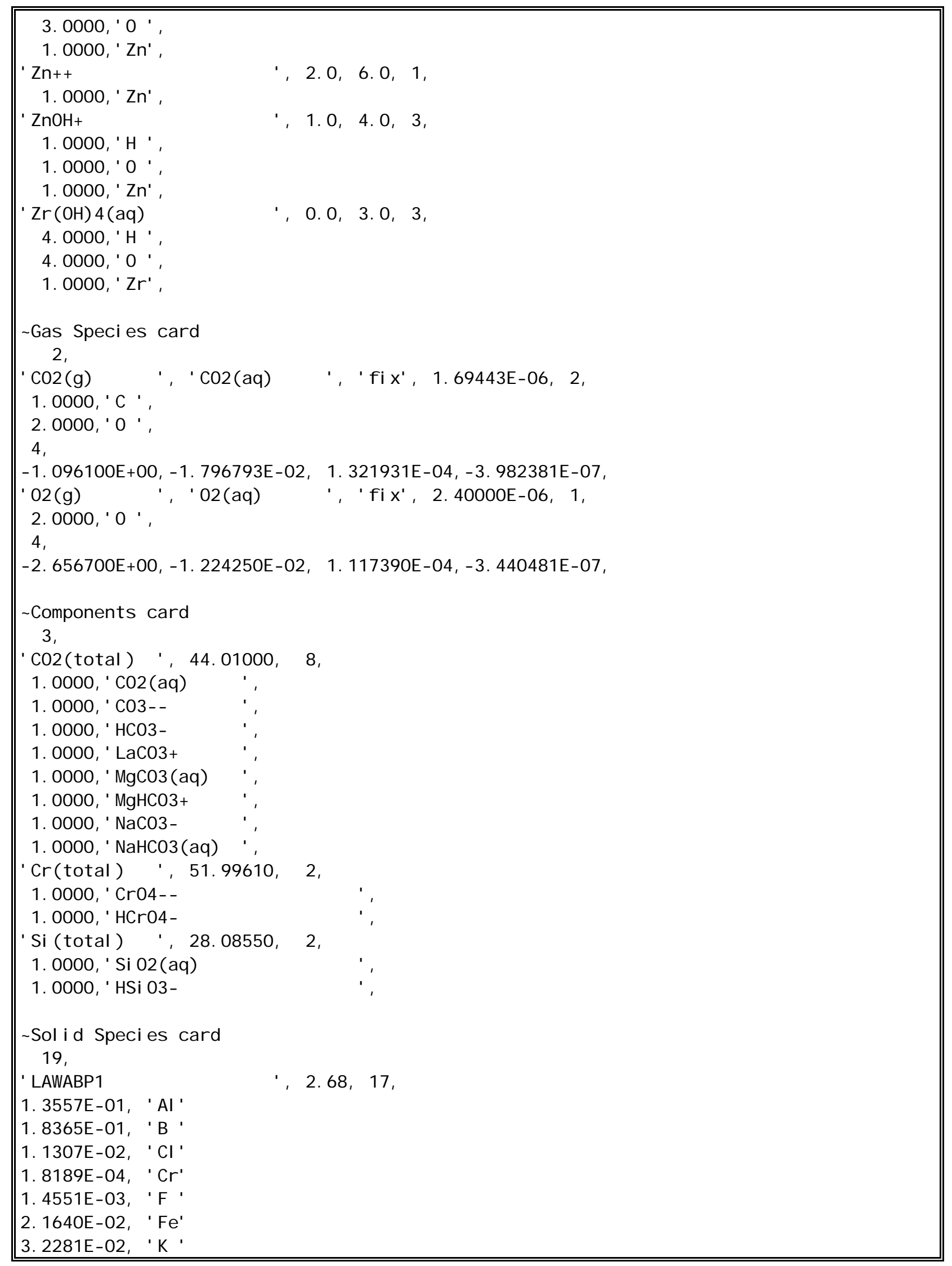




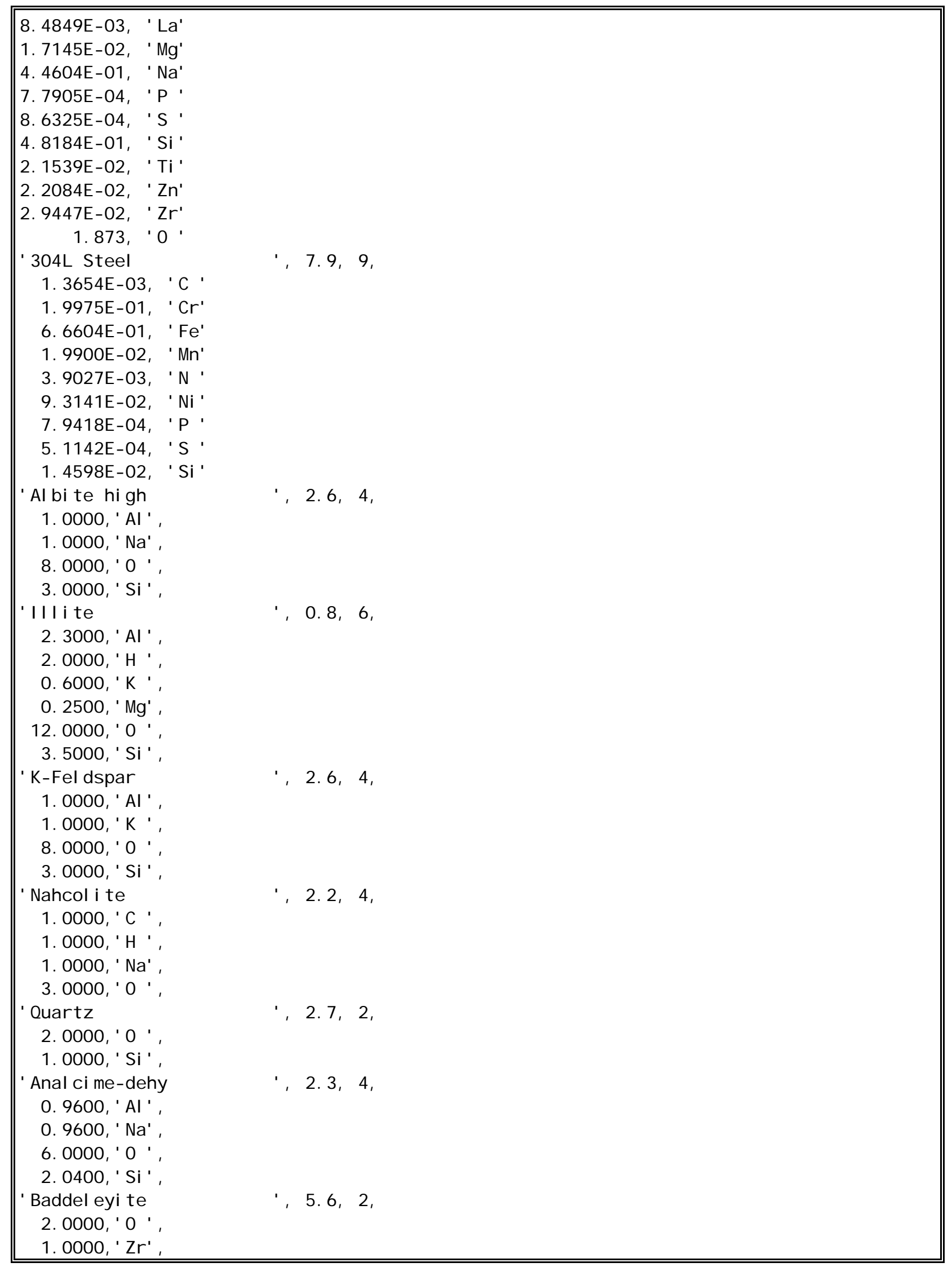




\begin{tabular}{|c|c|}
\hline 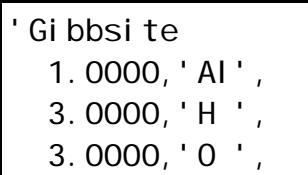 & $1,2.4,3$ \\
\hline $\begin{array}{l}\text { 'Goethite } \\
\text { 1.0000,' } \mathrm{Fe}^{\prime}, \\
1.0000, ' \mathrm{H}^{\prime}, \\
\text { 2.0000,' } 0 \text { ', }\end{array}$ & $', 4.3,3$ \\
\hline $\begin{array}{l}\text { 'Herschelite } \\
\text { 2. } 2600, \mathrm{Al}^{\prime}, \\
12.0000, \mathrm{H}^{\prime}, \\
0.5000, \mathrm{~K}^{\prime}, \\
1.6200, \mathrm{Na}^{\prime}, \\
18.4500, \mathrm{O}^{\prime}, \\
4.0000, \mathrm{Si}^{\prime},\end{array}$ & $1,1.8,6$ \\
\hline $\begin{array}{l}\text { La }(\mathrm{OH}) 3 \\
\quad 1.0000, ' \mathrm{La}^{\prime}, \\
3.0000,10{ }^{\prime}, \\
3.0000, ' \mathrm{H}^{\prime},\end{array}$ & $1,3,5,3$, \\
\hline $\begin{array}{r}\text { Nontronite- } \mathrm{Na} \\
0.3300, ' \mathrm{Al}^{\prime}, \\
2.0000, \mathrm{Fe}^{\prime}, \\
2.0000, \mathrm{H}^{\prime}, \\
0.3300, \mathrm{Na}^{\prime}, \\
12.0000, ' \mathrm{O}^{\prime}, \\
3.6700, ' \mathrm{Si}^{\prime},\end{array}$ & $1,2.3,6$ \\
\hline $\begin{array}{l}\text { 'Rutile } \\
\text { 2.0000, '0 ', } \\
\text { 1.0000, 'Ti', }\end{array}$ & ', 4.2, 2, \\
\hline $\begin{array}{l}\text { 'Sepiol ite } \\
14.0000, \mathrm{H}^{\prime}, \\
4.0000, \mathrm{Mg}^{\prime}, \\
23.0000, \mathrm{O}^{\prime}, \\
6.0000, \mathrm{Si}^{\prime},\end{array}$ & $1,2.3,4$, \\
\hline $\begin{array}{l}\text { 'si.02(am) } \\
2.0000, ' 0{ }^{\prime}, \\
1.0000, ' \mathrm{si}^{\prime},\end{array}$ & ' 2.1, 2, \\
\hline 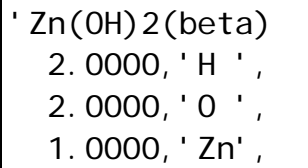 & $1,0.2,3$ \\
\hline 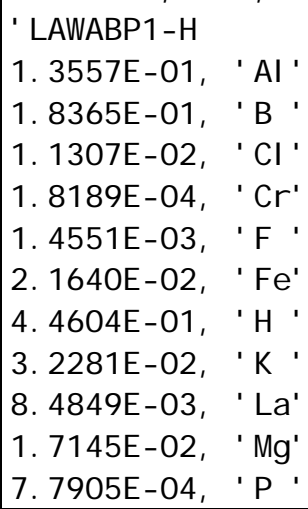 & ', 2.2745, 17, \\
\hline
\end{tabular}




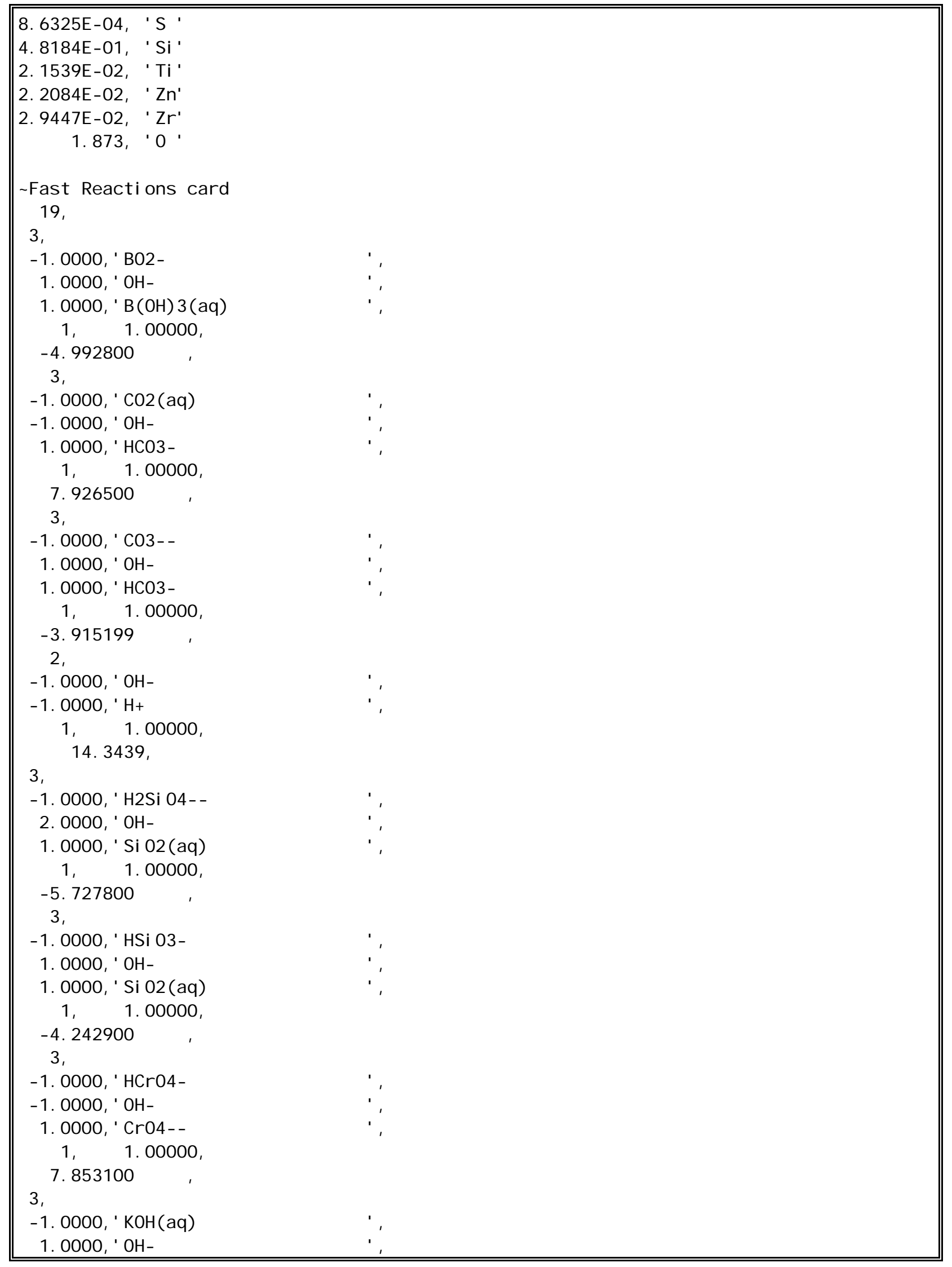




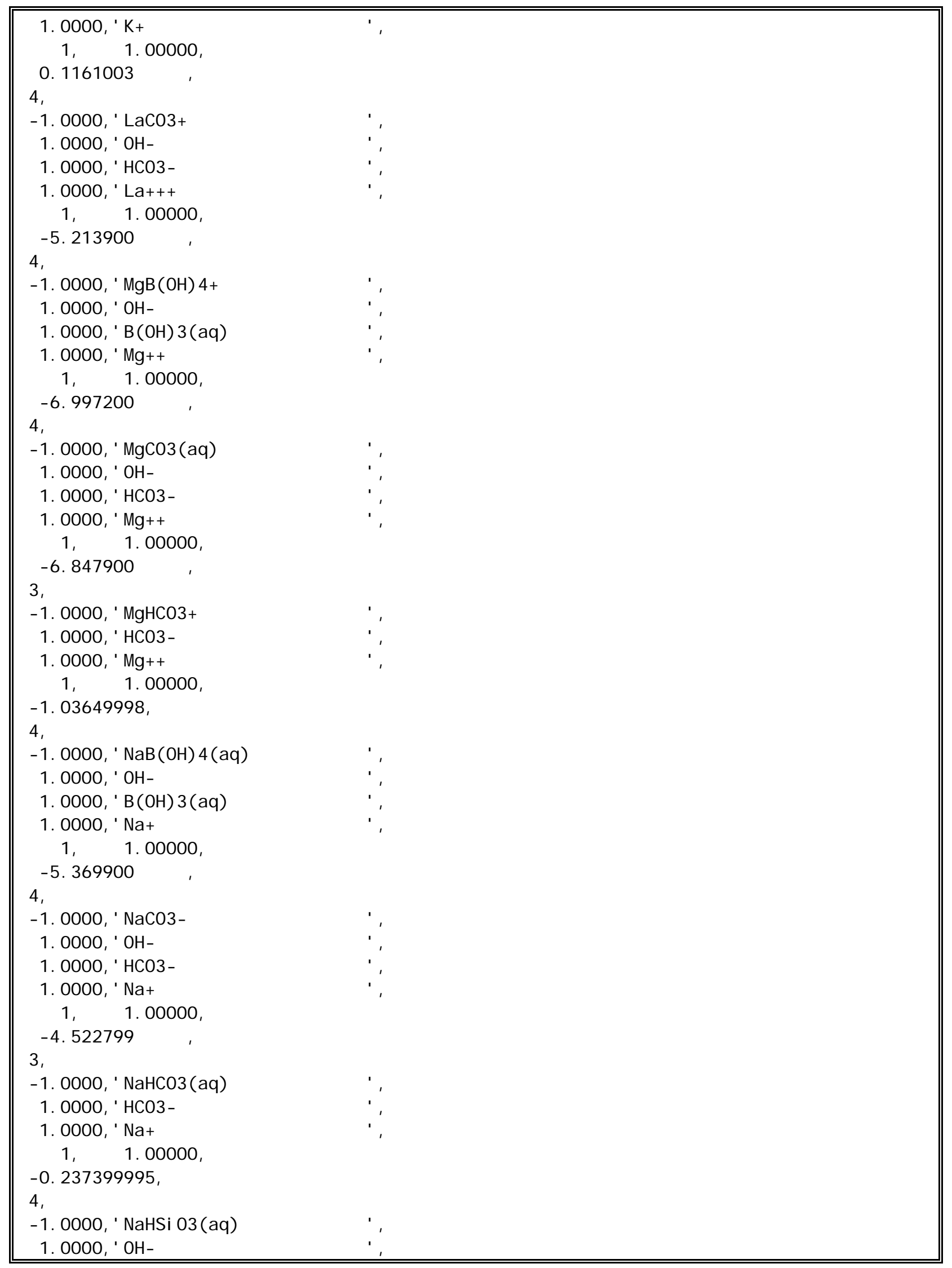




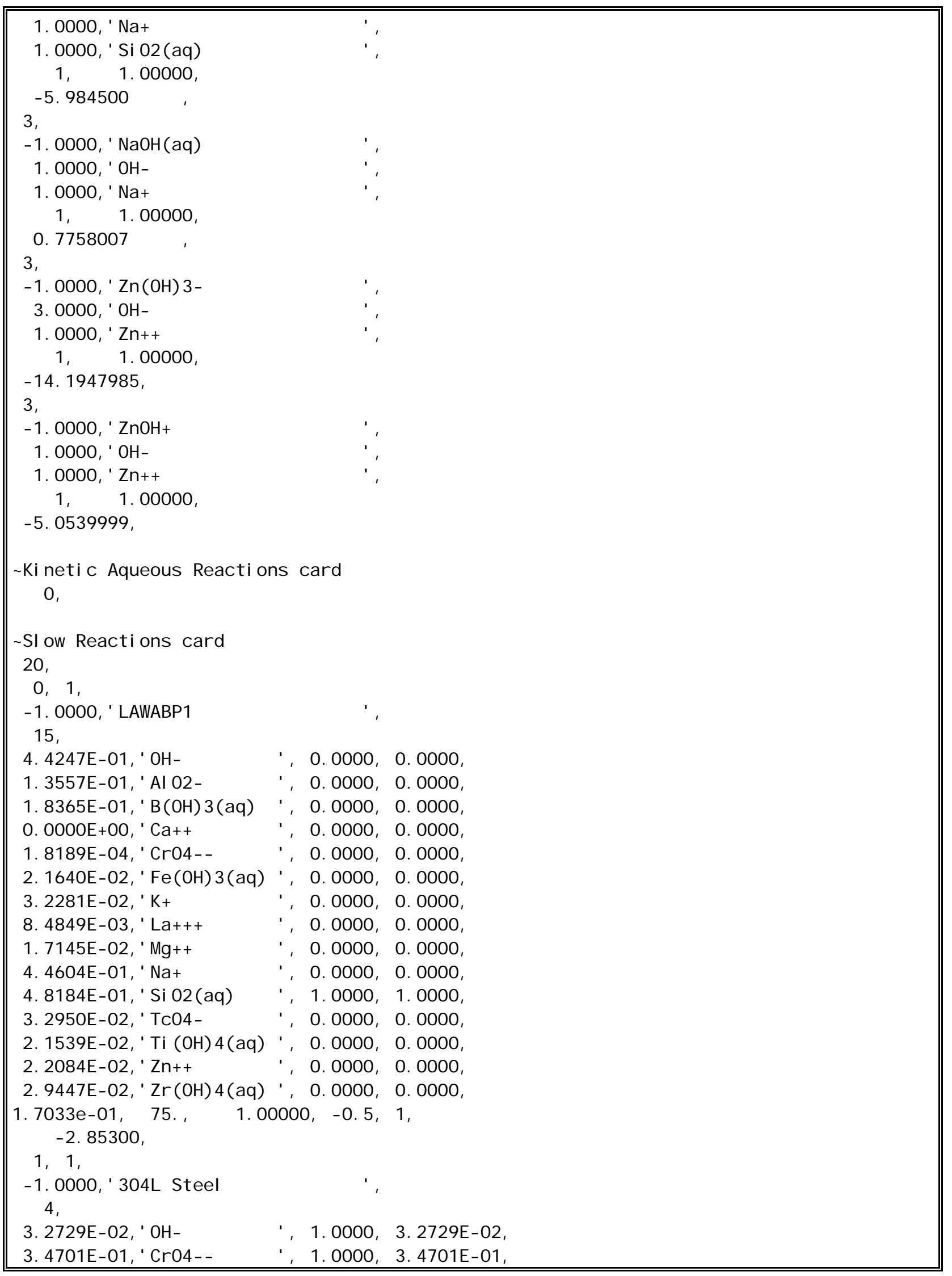




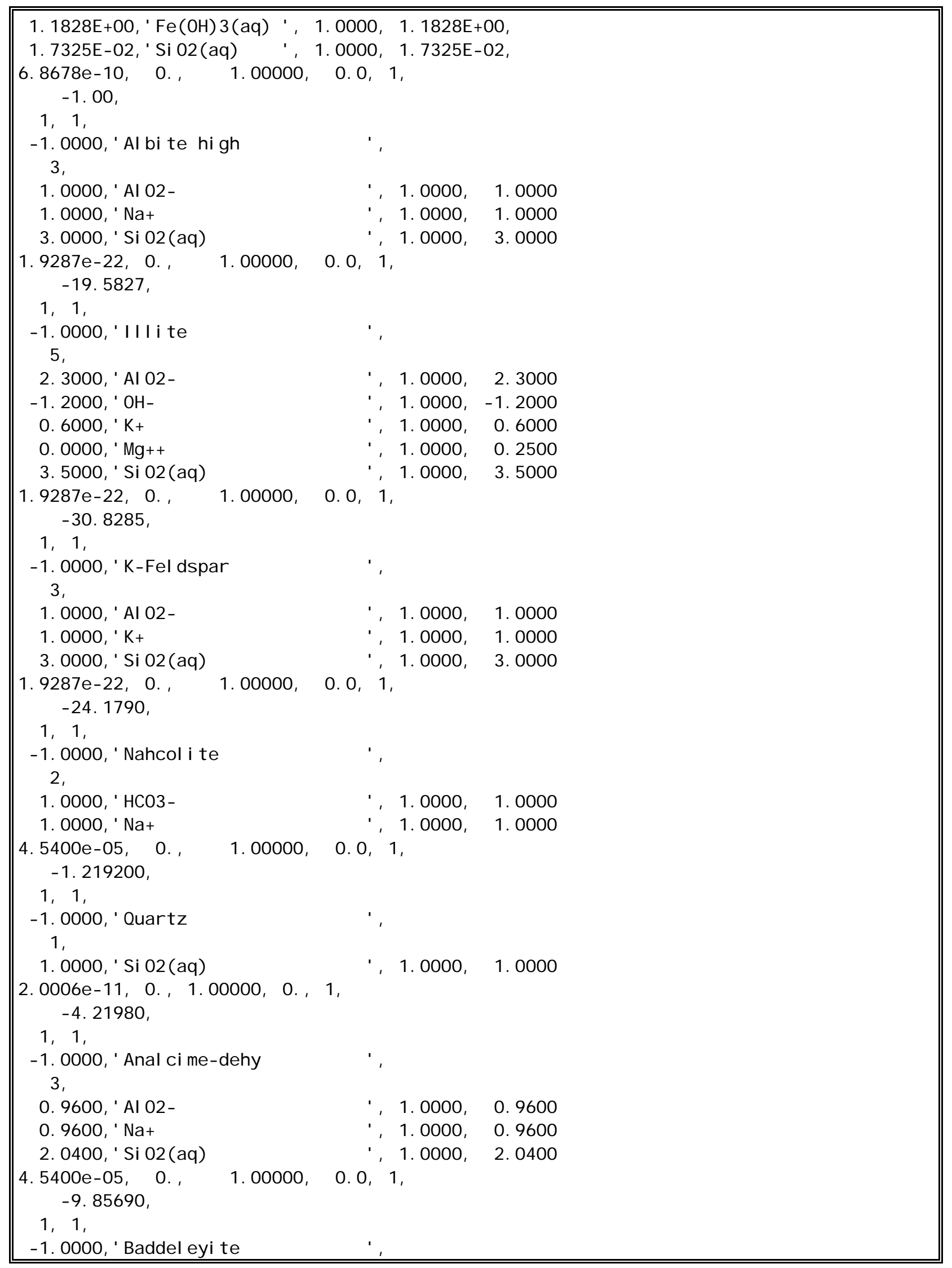




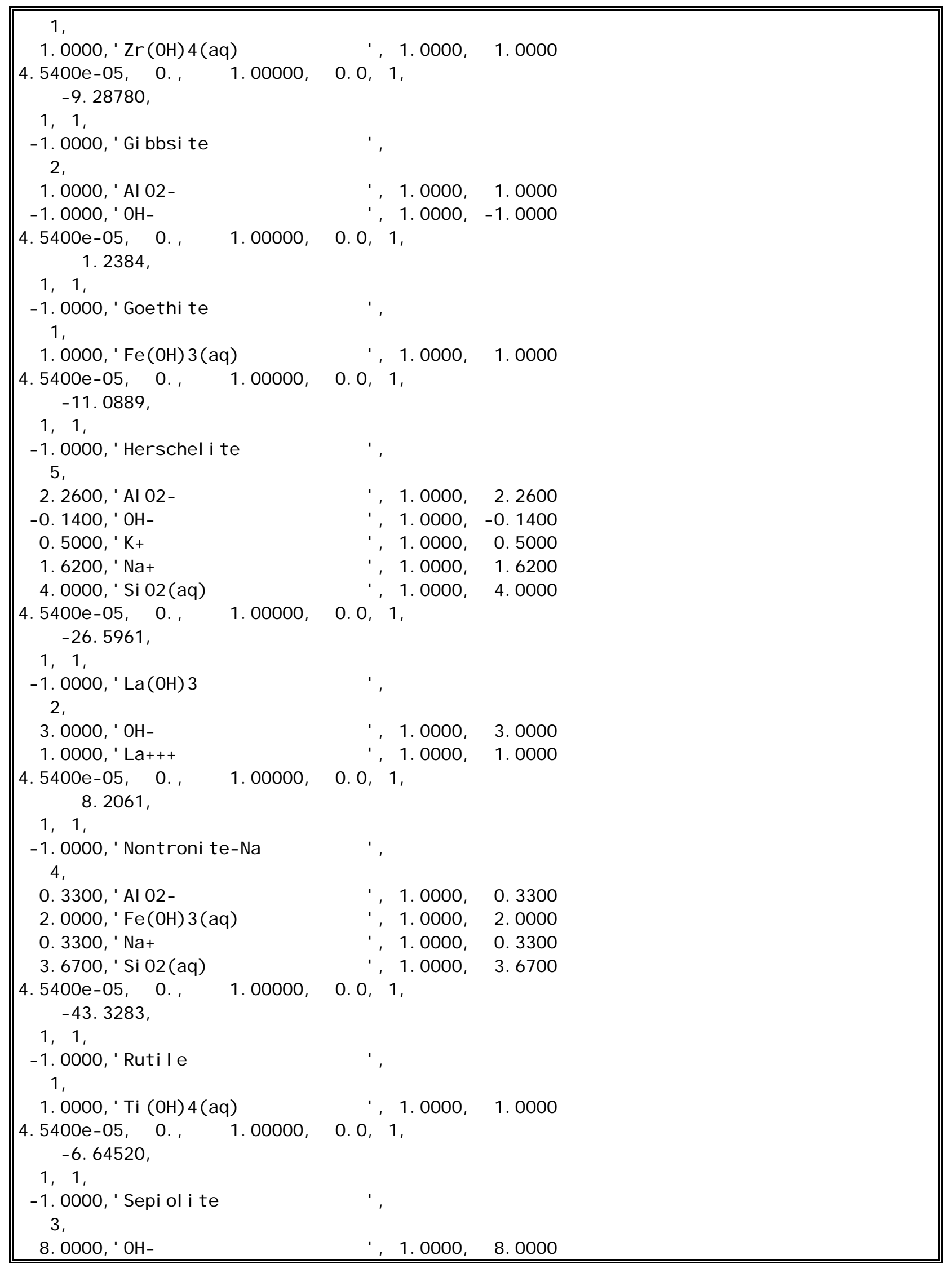




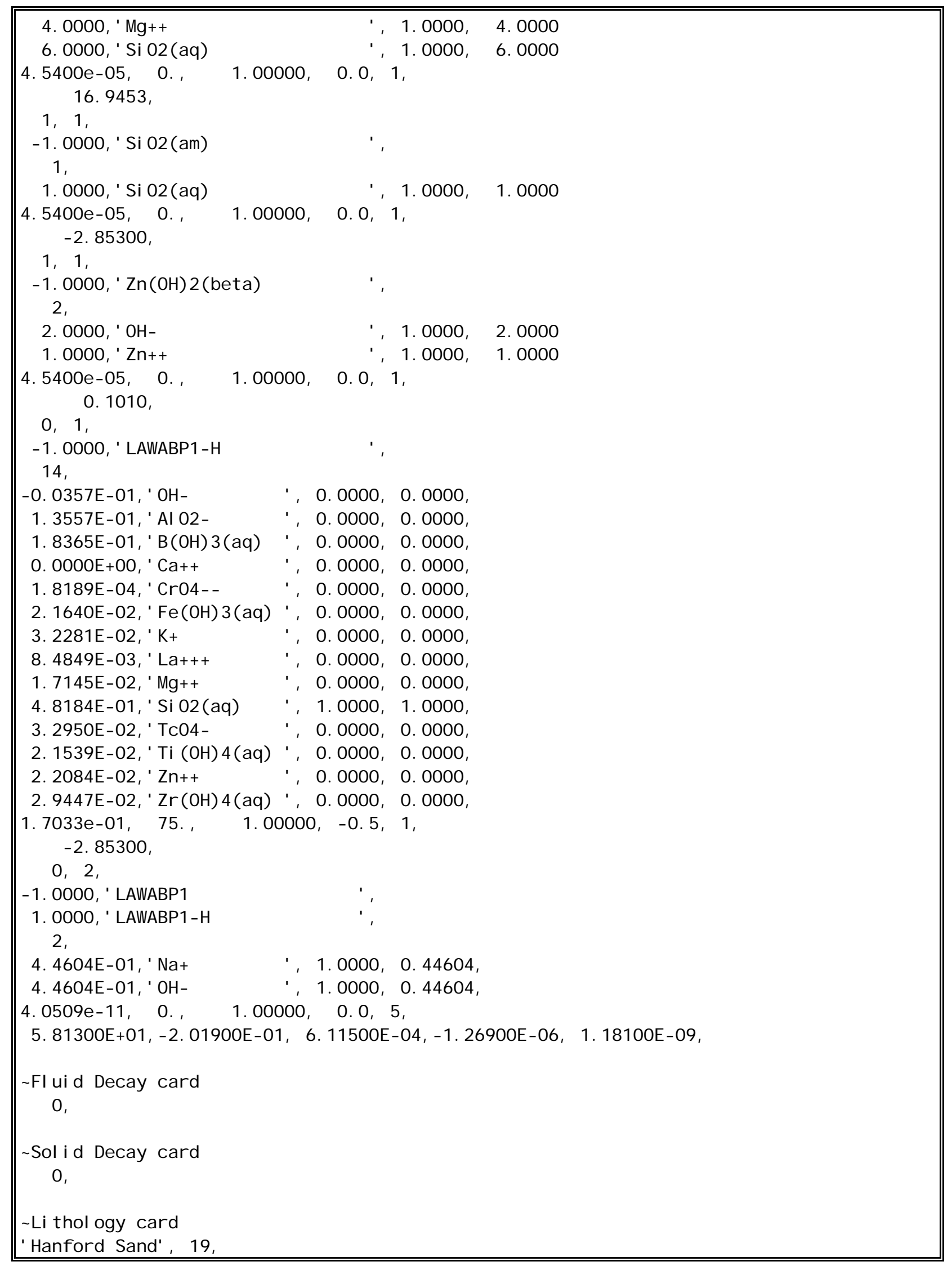




\begin{tabular}{|c|c|}
\hline ' LAWABP 1 & $\therefore, 0.00000 E+00,0.00000 E+00$, \\
\hline $1304 \mathrm{~L}$ Steel & $\therefore, 0.00000 E+00,0.00000 E+00$, \\
\hline Albite high & $\therefore \quad 0.400000+00,7.50000 E+03$, \\
\hline 'Illite & $\therefore \quad 0.10000 E+00,3.75000 E+04$ \\
\hline 'K-Feldspar & $\therefore 0.10000 E+00,1.87500 E+03$, \\
\hline I Nahcolite & $\therefore \quad 0.00001 E+00,-3.75000 E-03$, \\
\hline ' Quartz & $\therefore \quad 0.39987 \mathrm{E}+00, \quad 7.49756 \mathrm{E}+03$ \\
\hline 'Analci me-dehy & $\therefore \quad 0.00001 E+00,-3.75000 E-03$, \\
\hline 'Baddel eyi te & $\therefore \quad 0.00001 E+00,-3.75000 E-03$, \\
\hline ' Gibbsite & $\therefore \quad 0.00001 E+00,-3.75000 E-03$, \\
\hline ' Goethite & $\therefore \quad 0.00001 E+00,-3.75000 E-03$, \\
\hline ' Herschelite & $\therefore \quad 0.00001 E+00,-3.75000 E-03$, \\
\hline ' $\mathrm{La}(\mathrm{OH}) 3$ & $\therefore 0.00001 \mathrm{E}+00,-3.75000 \mathrm{E}-03$, \\
\hline ' Nontronite- $\mathrm{Na}$ & $\therefore 0.00001 E+00,-3.75000 E-03$, \\
\hline 'Rutile & $\therefore \quad 0.00001 E+00,-3.75000 E-03$, \\
\hline Sepiolite & $\therefore \quad 0.00001 \mathrm{E}+00,-3.75000 \mathrm{E}-03$, \\
\hline ' si 02 ( a m) & $\therefore 0.00001 E+00,-3.75000 E-03$, \\
\hline ( $\mathrm{Zn}(\mathrm{OH}) 2$ (bet a) & $\therefore \quad 0.00001 E+00,-3.75000 E-03$, \\
\hline ' L AWABP I- H & $\therefore \quad 0.00000 E+00,0.00000 E+00$, \\
\hline 'Backfill Soil', 19, & \\
\hline ' LAWABP 1 & $\therefore, 0.00000 E+00,0.00000 E+00$, \\
\hline $304 \mathrm{~L}$ Steel & $\therefore \quad 0.00000 E+00,0.00000 E+00$, \\
\hline Albite high & $\therefore \quad 0.40000 E+00,8.20800 E+03$, \\
\hline 'III ite & $\therefore \quad 0.10000 E+00,4.10400 E+04$ \\
\hline 'K-Feldspar & $\therefore \quad 0.10000 E+00,2.05200 E+03$ \\
\hline I Nahcolite & $\therefore \quad 0.00001 E+00,-4.10400 E-03$, \\
\hline ' Quartz & $\therefore \quad 0.39987 E+00,8.20533 E+03$ \\
\hline 'Anal ci me-dehy & $\therefore \quad 0.00001 E+00,-4.10400 E-03$, \\
\hline 'Baddel eyite & $\therefore \quad 0.00001 E+00,-4.10400 E-03$, \\
\hline 'Gibbsite & $\therefore \quad 0.00001 E+00,-4.10400 E-03$, \\
\hline 'Goethite & $\therefore \quad 0.00001 \mathrm{E}+00,-4.10400 \mathrm{E}-03$, \\
\hline ' Herschelite & $\therefore \quad 0.00001 E+00,-4.10400 E-03$, \\
\hline Lal $\mathrm{OH}) 3$ & $\therefore \quad 0.00001 E+00,-4.10400 E-03$, \\
\hline ' Nontronite-Na & $\therefore \quad 0.00001 \mathrm{E}+00,-4.10400 \mathrm{E}-03$, \\
\hline 'Rutile & $\therefore \quad 0.00001 \mathrm{E}+00,-4.10400 \mathrm{E}-03$, \\
\hline 'Sepiolite & $\therefore \quad 0.00001 E+00,-4.10400 E-03$, \\
\hline si 02 ( a m) & $\therefore \quad 0.00001 E+00,-4.10400 E-03$, \\
\hline ( $\mathrm{Zn}(\mathrm{OH}) 2$ (bet a) & $\therefore \quad 0.00001 E+00,-4.10400 E-03$, \\
\hline ' LAWABP $1-H$ & $\therefore 0.00000 E+00,0.00000 E+00$, \\
\hline ' Gl ass Waste ', 19, & \\
\hline ' LAWABP 1 & $1,0.87000 \mathrm{E}+00, \quad 4.38073 \mathrm{E}+01$, \\
\hline 1 $304 \mathrm{~L}$ Steel & $\therefore \quad 0.00000 E+00,0.00000 E+00$, \\
\hline 'Albite high & $\therefore \quad 0.00000 E+00,0.00000 E+00$, \\
\hline 'III ite & $\therefore \quad 0.00000 E+00,0.00000 E+00$, \\
\hline 'K-Feldspar & $\therefore \quad 0.00000 E+00, \quad 0.00000 E+00$, \\
\hline I Nahcolite & $\therefore \quad 0.00001 E+00,-5.88000 E-03$, \\
\hline ' Quartz & $\therefore \quad 0.00000 E+00,0.00000 E+00$, \\
\hline 'Analci me-dehy & $\therefore \quad 0.00001 E+00,-5.88000 E-03$, \\
\hline 'Baddel eyi te & $\therefore \quad 0.00001 E+00,-5.88000 E-03$, \\
\hline 'Gibbsite & $\therefore \quad 0.00001 E+00,-5.88000 E-03$, \\
\hline ' Goethite & $\therefore \quad 0.00001 E+00,-5.88000 E-03$, \\
\hline ' Herschelite & $\therefore \quad 0.00001 E+00,-5.88000 E-03$, \\
\hline $1 \mathrm{La}(\mathrm{OH}) 3$ & $\therefore, 0.00001 \mathrm{E}+00,-5.88000 \mathrm{E}-03$, \\
\hline
\end{tabular}




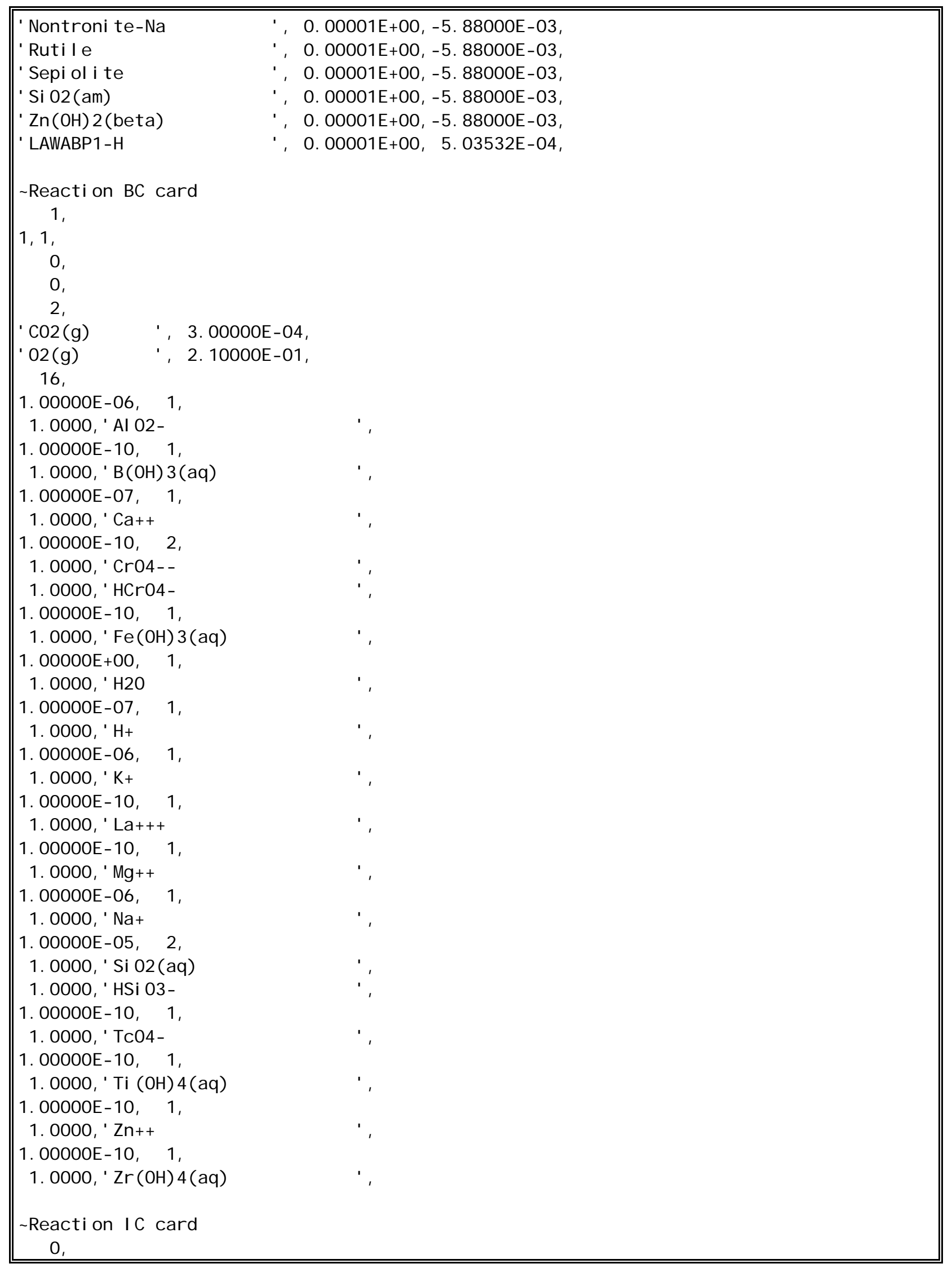




\section{Distribution}

No. of

Copies

OFFSITE

Argonne National Laboratory

Building 205

9700 South Cass Avenue

Argonne, IL 60439-4837

ATTN: WL Ebert

Lawrence Berkeley National Laboratory

Earth Sciences Division

University of California

Berkeley, CA 94720

ATTN: K Pruess

2 Lawrence Livermore National Laboratory

University of California

7000 East Avenue

Livermore, CA 94550-9234

ATTN: TJ Wolery, Mailcode L-219

WE Glassley, Mailcode L-202

University of Central Florida

322 Engineering Building I

Orlando, FL 32816-2450

ATTN: G-T Yeh

4 Yucca Mountain Site Characterization

Program

TRW Environmental Safety System

1180 Town Center Drive

Mail Stop 423

Las Vegas, NV 89134

ATTN: R Andrews

E Bartlett Mann

J Lee

Y Chen
No. of

Copies

ONSITE

7 CH2M Hill Group

MC Connelly E6-35

JG Field H6-62

AJ Knepp H6-03

FM Mann $\quad$ E6-35

B McMahon E6-45

G Parsons H6-19

R Raymond H6-22

3 U.S. Department of Energy/ORP

RW Lober H6-60

PE Lamont H6-60

RM Yasek H6-60

2 Fluor Daniel Hanford, Inc.

BH Ford E6-35

MI Wood H8-44

2 Fluor Federal Services, Inc.

R Khaleel B4-43

RJ Puigh B4-43

30 Pacific Northwest National Laboratory

LM Bagaasen K6-28

MP Bergeron K9-36

RW Bryce K6-75

DH Bacon (5) K9-33

WJ Deutsch K6-81

Y Fang K9-36 
No. of

Copies

MJ Fayer

VL Freedman

MD Freshley

CT Kincaid

KM Krupka

PE Long

BP McGrail (5)

WE Nichols

MC Richmond

ML Rockhold

KP Saripalli

TD Scheibe

RJ Serne

MD White

SB Yabusaki

F Zhang
K9-33

K9-36

K9-33

K9-33

K6-81

K9-33

K6-81

K9-33

K9-33

K9-36

K6-81

K9-36

P7-22

K9-36

K9-36

K9-33 